ISABELLA ANDRECZEVSKI CHAVES

\title{
OTIMIZAÇÃO DE PILARES DE CONCRETO ARMADO MEDIANTE UNIFORMIZAÇÃO DO ÍNDICE DE CONFIABILIDADE
}

Dissertação apresentada à Escola de Engenharia de São Carlos da Universidade de São Paulo, como parte dos requisitos para obtenção do título de Mestre em Engenharia de Estruturas.

ORIENTADOR: $\operatorname{Prof}^{\mathrm{a}} \mathrm{Dr}^{\mathrm{a}}$ Ana Lúcia H. C. El Debs 
Dedico este trabalho e os méritos desta conquista a minha família. 


\section{AGRADECIMENTOS}

Agradecer é admitir que houve um momento em que se precisou de alguém, é reconhecer que o homem jamais poderá lograr para si o dom de ser auto-suficiente. Ninguém e nada crescem sozinhos; sempre é preciso um olhar de apoio, uma palavra de incentivo, um gesto de compreensão, uma atitude de amor. A todos que me auxiliaram nesse trabalho agradeço com a mais profunda gratidão e respeito, e em especial:

A Deus, por estar sempre presente em minha vida.

A Professora Ana Lúcia H. C. El Debs pela orientação, apoio, paciência, compreensão e confiança depositada em mim.

Ao Professor Wilson Sergio Venturini pela contribuição significativa a elaboração deste trabalho.

Ao Professor, pai e amigo Anselmo Chaves Neto, agradeço por ter me mostrado um caminho a seguir. Obrigado por me abrir os olhos e me fazer acreditar mais em mim, na vida e no futuro.

A minha mãe Sonia, agradeço por estar sempre ao meu lado.

As minhas irmãs Paula e Helena, pelo apoio, incentivo e paciência nos momentos difíceis.

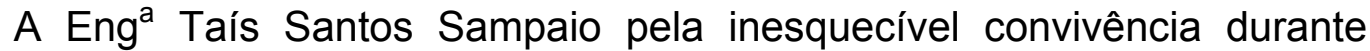
nossa estadia em São Carlos.

A Eng ${ }^{a}$ Lizandra Nogami pela amizade, apoio e incentivo, nunca negados. 
Ao Eng. André Velame, agradeço pelos valiosos comentários, sugestões, dedicação e paciência para suportar minhas insistentes consultas.

A alguns dos meus familiares, agradeço pelo incentivo e preocupação.

Aos amigos que deixei em Curitiba e aos amigos que fiz em São Carlos, agradeço pelo incentivo e companheirismo.

Aos professores Marcos Antonio Marino e Roberto Dalledone Machado que além de mestres foram amigos e em sua amizade me incentivaram a seguir o caminho científico.

Aos professores, funcionários e colegas do SET que contribuíram de forma direta ou indiretamente para a elaboração desse trabalho.

A CAPES, pela bolsa de estudo concedida, sem a qual não seria possível a realização deste trabalho. 


\section{SUMÁRIO}

RESUMO ..V

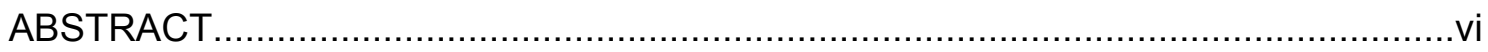

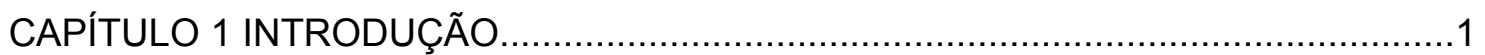

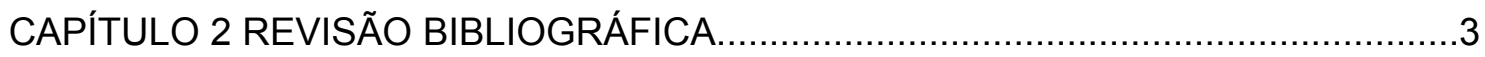

2.1 ‘ESTADO-DA-ARTE’ DA PESQUISA EM OTIMIZAÇÃO .............................................. 3

2.2 ‘ESTADO-DA-ARTE’ DA PESQUISA EM CONFIABILIDADE ...................................... 11

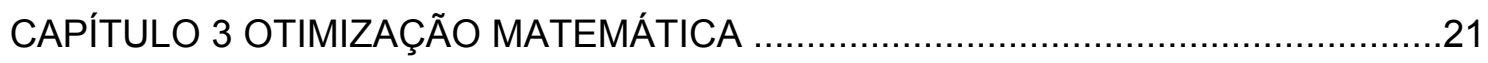

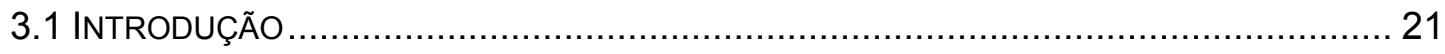

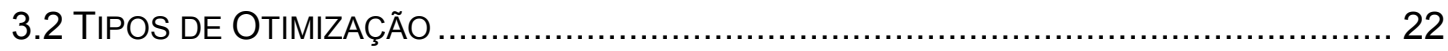

3.2.1 Otimização Discreta e Otimização Contínua ............................................. 22

3.2.2 Otimização Restrita e Otimização Não-Restrita ........................................ 22

3.2.3 Otimização Local e Otimização Global.................................................. 23

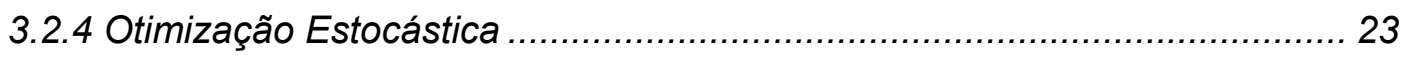

3.3 FormulaÇÃo MATEMÁtICA de PROBlemas de OtIMIZAÇÃo ................................ 23

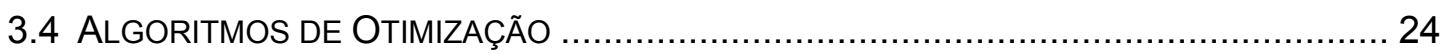

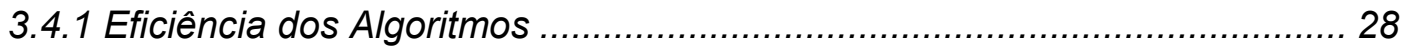

3.5 FUNDAMENTOS MATEMÁTICOS PARA OTIMIZAÇÃO ............................................ 29

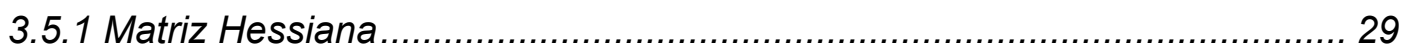

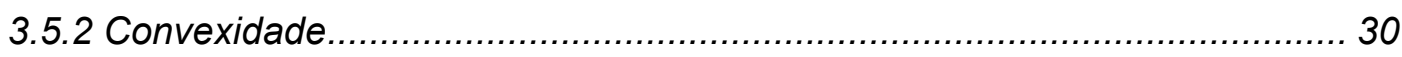

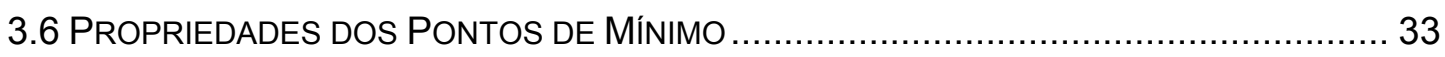

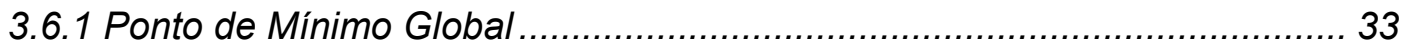

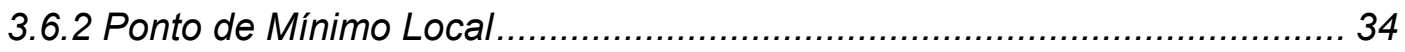

3.7 FUNDAMENTOS DA OTIMIZAÇÃO NÃO-RESTRITA ............................................ 40

3.8 FUNDAMENTOS DA OTIMIZAÇÃO RESTRITA .............................................. 40

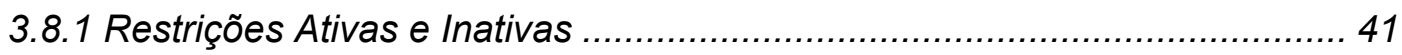


3.8.2 Método dos Multiplicadores de Lagrange.......................................... 42

3.8.3 Condições para Mínima Restrição ...................................................... 43

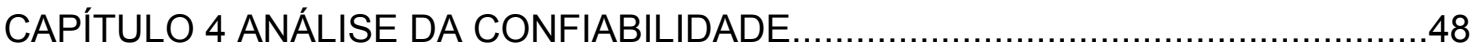

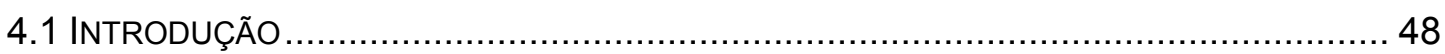

4.2 FUNDAMENTOS ESTATÍSTICOS DA CONFIABILIDADE ....................................... 49

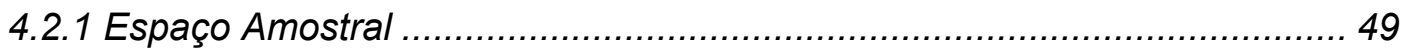

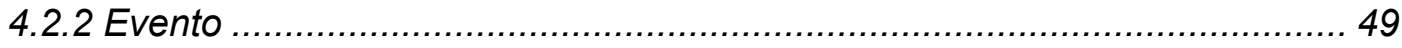

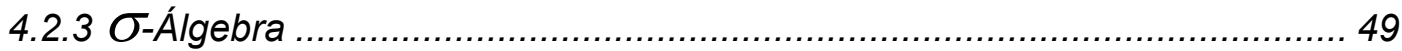

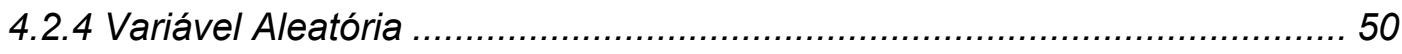

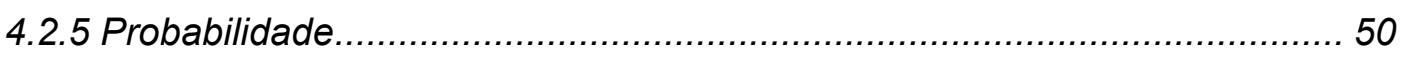

4.2.5.1 Propriedades da Probabilidade .................................................... 50

4.2.5.2 Probabilidade Condicional e Independência de Eventos .................... 51

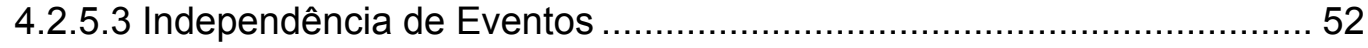

4.2.5.4 Teorema da Probabilidade Total ................................................. 54

4.2.5.5 Função Distribuição ............................................................... 54

4.2.5.6 Função de Probabilidade e Função Densidade de Probabilidade........ 55

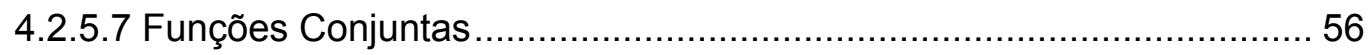

4.2.5.8 Distribuição de Probabilidade Normal (Gaussiana) ........................... 57

4.2.5.9 Distribuição de Soma e Diferença de Duas Variáveis Aleatórias

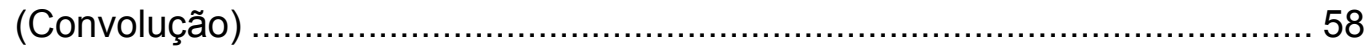

4.2.6 Esperança e Variância de uma Variável Aleatória ................................. 59

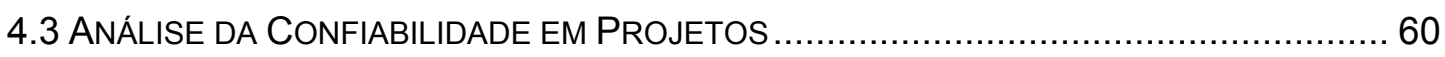

4.3.1 Confiabilidade em Sistemas Estruturais............................................ 60

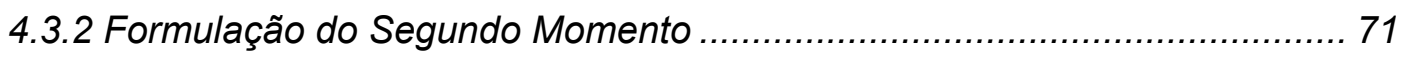

4.3.3 Funções de Desempenho Lineares................................................. 80

CAPÍTULO 5 MÉTODO DE SUPERFÍCIE DE RESPOSTA......................................84

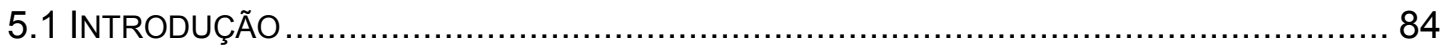




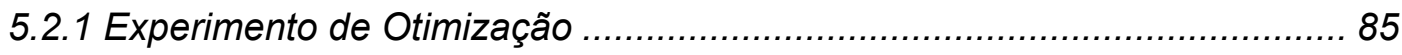

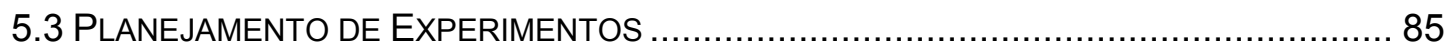

5.3.1 Aplicação das Técnicas de Planejamento de Experimentos...................... 85

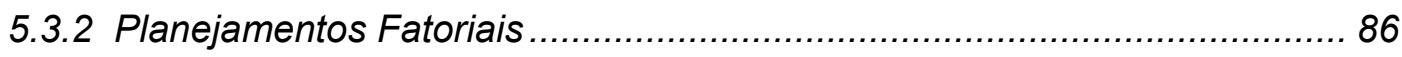

5.4 Métodos e Planejamentos de Superfí́cie de Resposta .............................. 86

5.4.1 Método da Ascendente de Maior Inclinação (Steepest Ascent).................. 89

5.4.2 Análise de uma Superfície de Resposta de Segunda Ordem ................... 90

5.4.2.1 Modelo de Regressão Linear Múltipla ........................................... 90

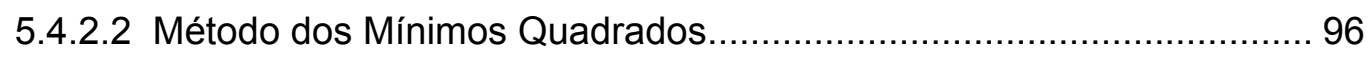

5.4.2.3 Abordagem Matricial para a Regressão Linear Múltipla...................... 98

5.5 DeSENVOLVIMENTO dA SUPERFÍCIE DE RESPOSTA ......................................... 100

5.5.1 Definição do Espaço de Trabalho ..................................................... 101

5.5.2 Transformações de Distribuições de Probabilidade ................................ 101

5.5.2.1 Variáveis Aleatórias Independentes ........................................ 102

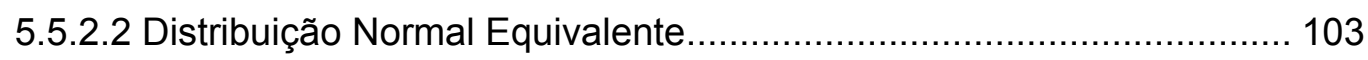

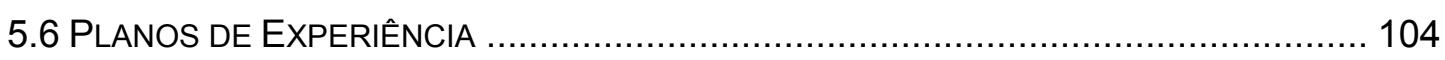

5.6.1 Plano de Experiência Numérico Estrela ........................................... 107

5.6.2 Plano de Experiência Numérico Hiper-cubo ou Estrela 45 ...................... 110

5.6.3 Plano de Experiência Numérico Fatorial Completo................................ 113

5.6.4 Plano de Experiência Numérico Mínimo ............................................... 116

5.6.5 Plano de Experiência Numérico Composto......................................... 119

5.6.6 Planos de Experiência Aleatórios................................................. 124

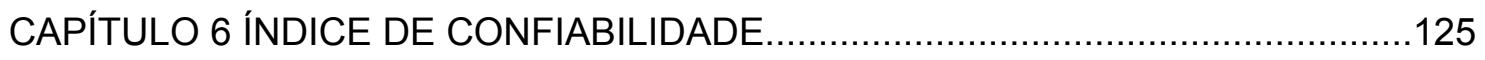

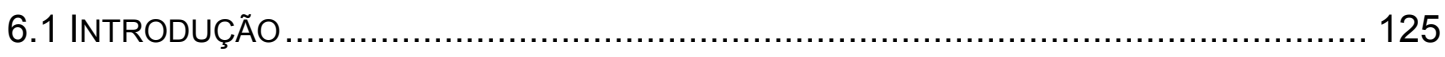

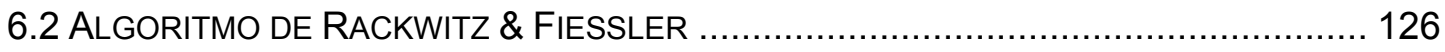

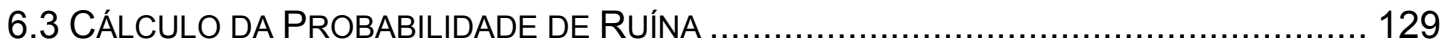

6.3.1 Método de Confiabilidade de Primeira Ordem - FORM ......................... 129 
6.3.2 Método de Confiabilidade de Segunda Ordem - SORM.......

6.3.2.1 Aproximação por uma Hiper-esfera ............................................... 133

6.3.2.2 Hiper-esfera Excêntrica ................................................................. 133

6.3.2.3 Aproximação Assintótica ......................................................... 134

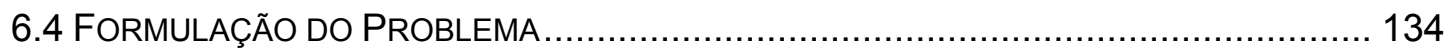

CAPÍTULO 7 OTIMIZAÇÃO DA SEÇÃO TRANSVERSAL DE UM PILAR..................137

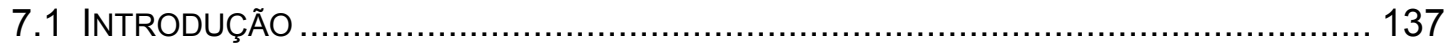

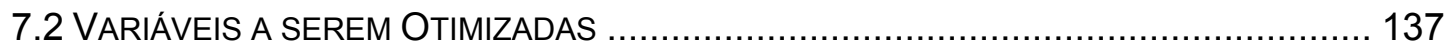

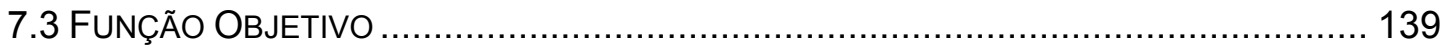

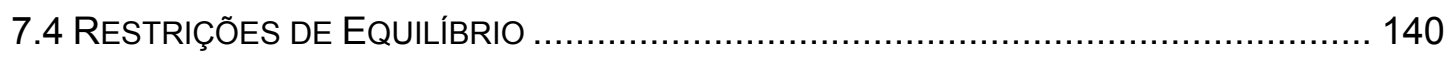

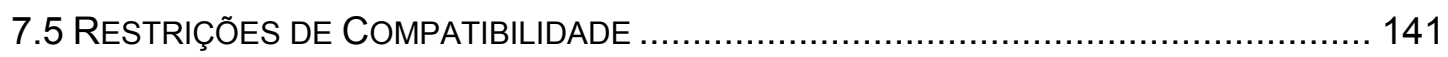

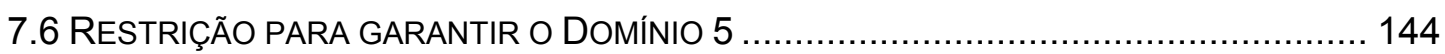

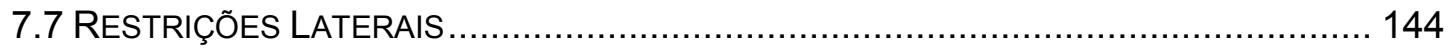

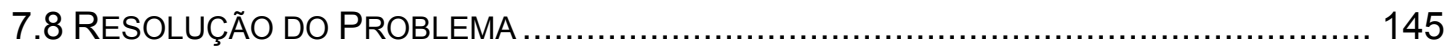

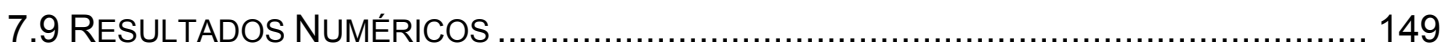

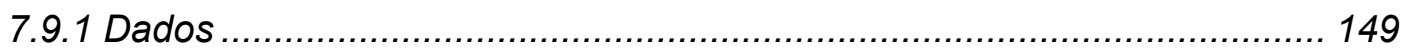

7.9.1.1 Resistências Características dos Materiais: ................................... 149

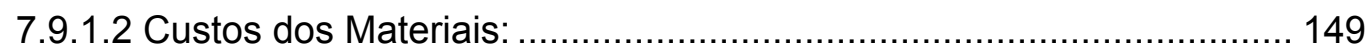

7.9.1.3 Valores Limites das Variáveis:.................................................... 149

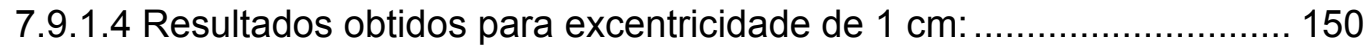

7.9.1.5 Resultados obtidos para excentricidade de $2 \mathrm{~cm}$ :......................... 151

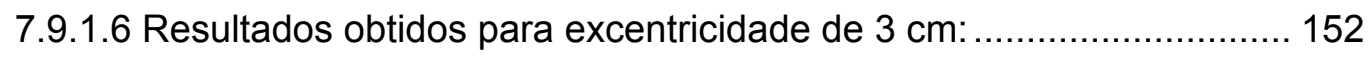

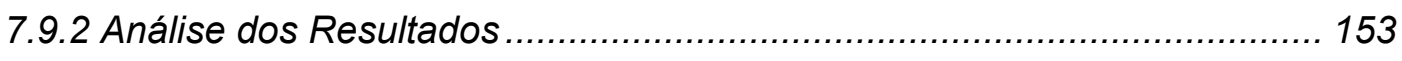

CAPÍTULO 8 CÁLCULO DO ÍNDICE DE CONFIABILIDADE..................................154

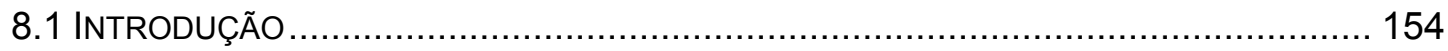

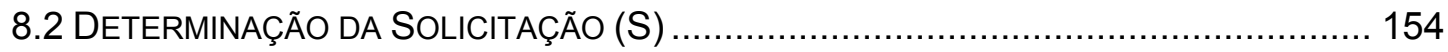

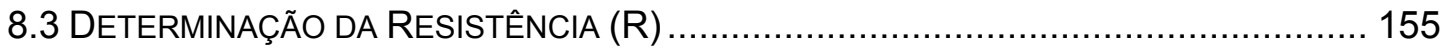

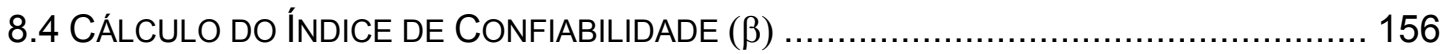




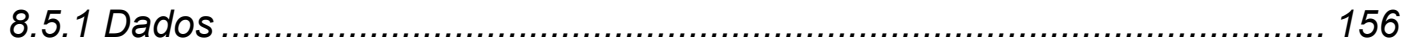

8.5.1.1 Características Determinísticas .................................................... 156

8.5.1.2 Características Estatísticas ....................................................... 157

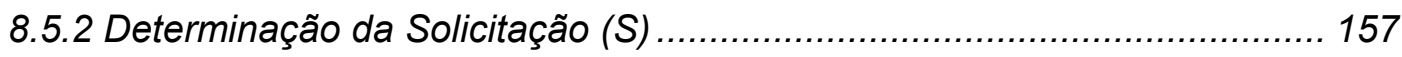

8.5.3 Determinação do Plano de Experiência (PE) ......................................... 157

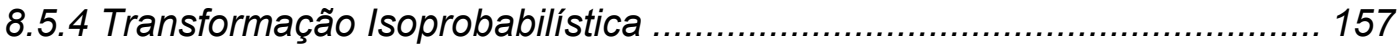

8.5.5 Determinação do Polinômio da SR ................................................... 158

8.5.6 Determinação do Índice de Confiabilidade $(\beta)$....................................... 158

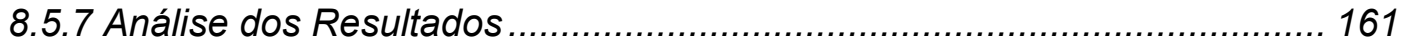

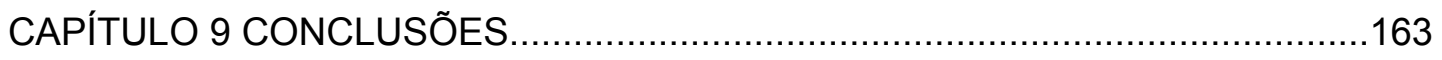

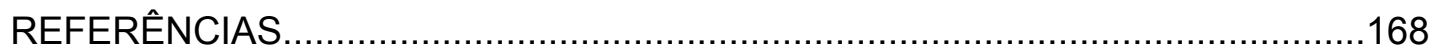


FIGURA 3.1 - Representação geométrica de um problema de otimização. .24

FIGURA 3.2 - Exemplo de um conjunto convexo. 31

FIGURA 3.3 - Exemplo de um conjunto não convexo. 32

FIGURA 3.4 - Exemplo de união de conjuntos convexos... .33

FIGURA 3.5 - Exemplo de interseção de conjuntos não-convexos. 33

FIGURA 3.6 - Exemplo de uma função com um mínimo global e dois mínimos locais. .35

FIGURA 3.7 - Exemplo de restrições ativas e inativas. 41

FIGURA 4.1 - Partição do espaço amostral $\Omega$ .53

FIGURA 4.2 - Partição do espaço amostral $\Omega$...... 53

FIGURA 4.3 - Função distribuição acumulada. 55

FIGURA 4.4 - Função densidade de probabilidade da Normal Padrão. .56

FIGURA 4.5 - f.d.p.'s $f_{X}(x)$ e $f_{Y}(y)$. 64

FIGURA 4.6 - Efeito da posição relativa entre $f_{X}(x)$ e $f_{Y}(y)$ em $p_{F}$ 65

FIGURA 4.7 - Efeito das dispersões em $f_{X}(x)$ e $f_{Y}(y)$ em $p_{F}$. 65

FIGURA 4.8 - f.d.p. da margem de segurança M. 68

FIGURA 4.9 - f.d.p. do fator de segurança FS .71

FIGURA 4.10 - Espaço das variáveis padronizadas $Z_{X}$ e $Z_{Y}$. .73

FIGURA 4.11 - Superfície linear do estado limite no espaço $z_{x_{1}}, z_{x 2}, z_{x 3}$. .81

FIGURA 5.1 - Exemplo de um plano de regressão. 91

FIGURA 5.2 - Exemplo de curvas de nível. 92

FIGURA 5.3 - Superfície de um modelo de regressão múltipla. .94

FIGURA 5.4 - Curvas de nível para um modelo de regressão múltipla. .94

FIGURA 5.5 - Superfície de um modelo de regressão múltipla..... .95

FIGURA 5.6 - Curvas de nível para um modelo de regressão múltipla. .96 
FIGURA 5.7 - Planos de experiência numéricos adotados no presente trabalho. 105

FIGURA 6.1 - Interpretação gráfica do FORM no caso de apenas 1 variável aleatória. 131

FIGURA 6.2 - Sinal do erro na aproximação FORM. 132

FIGURA 6.3 - Interpretação gráfica do problema de confiabilidade estrutural. 136

FIGURA 7.1 - Denominação das variáveis a serem otimizadas. 138

FIGURA 7.2 - Condições de equilíbrio. 140

FIGURA 7.3 - Diagrama de deformações para o Domínio 5. 142

FIGURA 7.4 - Diagrama tensão x deformação para o aço CA-50 A 143 


\section{LISTA DE TABELAS}

TABELA 4.1- Valores de $\beta$ e da probabilidade de falha $p_{F}$ .70

TABELA 5.1 - Número de coeficientes em relação ao número de v.a.......................106

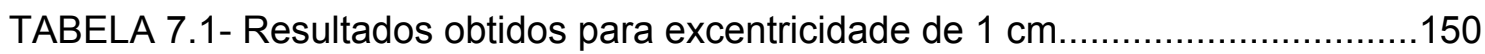

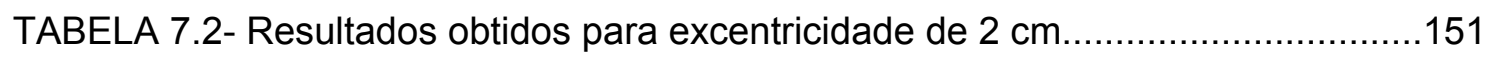

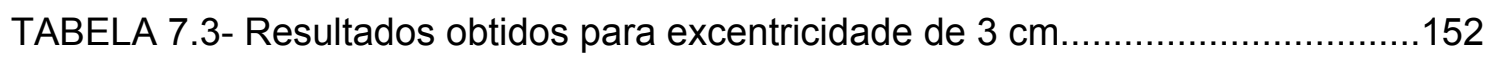

TABELA 8.1- Índices de confiabilidade para as seções ótimas................................161

TABELA 8.2- Índices de confiabilidade para as seções ótimas................................162 


\section{LISTA DE GRÁFICOS}

GRÁFICO 7.1- Resultados obtidos para excentricidade de $1 \mathrm{~cm}$. 150

GRÁFICO 7.2- Resultados obtidos para excentricidade de $2 \mathrm{~cm}$. 151

GRÁFICO 7.3- Resultados obtidos para excentricidade de $3 \mathrm{~cm}$. 152 


\section{RESUMO}

CHAVES, I.A. (2004). Otimização de pilares de concreto armado mediante uniformização do índice de confiabilidade. Dissertação (Mestrado) - Escola de Engenharia de São Carlos, Universidade de São Paulo, São Carlos, 2004.

Atualmente, busca-se a minimização dos custos para uma estrutura que satisfaça a princípios de segurança, com respeito aos estados limites de ruptura e de utilização, e as imposições de normas técnicas. Entretanto, nenhuma estrutura é totalmente segura. $O$ projeto envolve incertezas, pois há variações tanto nos carregamentos quanto na capacidade resistente e rigidez da estrutura. Deste modo, modelos de cálculo que se baseavam em hipóteses bastante simplificadoras estão sendo substituídos por modelos que consideram as variabilidades dos parâmetros estruturais e das solicitações, conseqüentemente, obtém-se uma avaliação mais confiável e realista da capacidade última da estrutura. Na prática, em estruturas que atendem aos requisitos básicos de segurança, dificilmente o grau de economia é verificado. Assim, através de técnicas de otimização matemática e de conceitos estatísticos, um programa computacional foi desenvolvido para a determinação da seção transversal de pilares de concreto armado com o custo mínimo e a determinação do índice de confiabilidade desta estrutura para um estado limite.

Palavras-chave: otimização; estruturas; pilares; concreto armado; confiabilidade; estatística 
ABSTRACT

CHAVES, I.A. (2004). Optimization of Reinforced Concrete Columns and Calculation of the Index of Reliability. M.Sc. Dissertation - School of Engineering of São Carlos, University of São Paulo, São Carlos, 2004.

Currently, it searches minimization of the costs for a structure that satisfies the security principles, with respect to the states use and rupture limits, and the impositions of norms techniques. However, no structure is total insurance. The project involves uncertainties, therefore it in such a way has variations in loading how much in the resistant capacity and rigidity of the structure. In this way, calculation models that if based on sufficiently simple hypotheses are being substituted for models that consider the variabilities of the structural parameters and the requests, consequently, get a more reliability and realistic evaluation of the last capacity of the structure. In the practical one, in structures that take care of to the basic requirements of security, hardly the economy degree is verified. Thus, through techniques of mathematical optimization and statistical concepts, a computational program was developed for the determination of the cross section of columns of reinforced concrete with the minimum cost and the determination of the index of reliability of this structure for a state has limited.

Keywords: optimization; structures; columns; reinforced concrete; reliability; statistics 


\section{INTRODUÇÃO}

Incertezas são inevitáveis no projeto estrutural, pois há variações tanto nos carregamentos quanto na capacidade resistente e rigidez da estrutura. Portanto, os procedimentos de análise da engenharia devem, adequadamente, incluir métodos e conceitos para avaliação de sua importância no desempenho e no projeto do sistema. Com essa consideração, os princípios de probabilidade, estatística e teoria da decisão oferecem a base matemática para a modelagem da incerteza e análise dos seus efeitos no projeto.

A utilização de processos que levem em consideração a natureza aleatória das variáveis envolvidas nos modelos de comportamento do concreto armado é uma ferramenta indispensável e talvez até a única realmente apropriada para a estimativa da confiabilidade nas estruturas.

Com isso, o esquema do projeto estrutural está melhorando no que se refere à introdução de novos modelos nas normas. Deste modo, modelos de cálculo que se baseavam em hipóteses bastante simplificadoras estão sendo substituídos por modelos que consideram as variabilidades dos parâmetros estruturais e das solicitações, conseqüentemente, obtém-se uma avaliação mais confiável e realista da capacidade última da estrutura.

Atualmente, busca-se a minimização dos custos para uma estrutura que satisfaça a princípios de segurança, com respeito aos estados limites de ruptura e de utilização, e as imposições de normas técnicas. Entretanto, em estruturas que atendem aos requisitos básicos de segurança, dificilmente 0 grau de economia é verificado. 
Assim, através de técnicas de otimização matemática e de conceitos estatísticos, um programa computacional foi desenvolvido para a determinação da seção transversal de pilares de concreto armado de forma a terem custo mínimo e no cálculo da confiabilidade desta estrutura para um estado limite. 


\section{REVISÃO BIBLIOGRÁFICA}

\section{1 'Estado-da-arte’ da Pesquisa em Otimização}

De acordo com VIANNA (2003), o objetivo principal do projeto estrutural é encontrar o ponto de equilíbrio entre a maximização da segurança e a minimização dos custos. Como os dois conceitos são contraditórios entre si, na prática se busca uma minimização dos custos para uma estrutura que satisfaça a princípios básicos de segurança, com respeito aos estados limites de ruptura e de utilização, e as imposições de normas técnicas. Sucintamente, pode-se definir otimização estrutural como sendo a definição de uma série de variáveis de projeto que juntas vão extremar uma função objetivo definida.

Segundo CAMP et al. (1998), as técnicas de otimização podem ser divididas em três categorias: programação matemática, método dos critérios de otimização e os métodos heurísticos ou métodos genéticos.

A programação matemática pode ser dividida em programação linear e não-linear. $\mathrm{Na}$ programação linear, a função objetivo e as restrições são funções lineares das variáveis de projeto. A programação não-linear foi desenvolvida para problemas de otimização onde as restrições são funções não-lineares das variáveis de projeto, e as condições necessárias para alcançar a solução ótima são providas pelas condições de KUHN e TUCKER (1951).

Como a aplicação direta das condições de Kuhn-Tucker é extremamente difícil para a maioria dos problemas, o método dos critérios de otimização foi criado com contribuições de BARNETT (1961), PRAGER (1968) e VENKAYYA et al. (1968), para a sua aplicação indireta, combinadas com os 
multiplicadores de Lagrange nos problemas de programação não-linear. Este método vem sendo usado na maioria dos problemas de engenharia de estruturas, como em RIZZI (1976), ARORA (1980), MOHARRAMI e GRIERSON (1993), SOARES (1997) e VIANNA (2003).

O algoritmo genético é uma técnica baseada nos princípios genéticos de sobrevivência de uma população através de adequações e adaptações. 0 método dos algoritmos genéticos tem sido empregado com sucesso na otimização estrutural, como em GOLDBERG e SAMTANI (1986) e JENKINS (1992).

Desde o trabalho pioneiro de SCHIMIDT (1960), a teoria da otimização estrutural tem avançado consideravelmente nas últimas quatro décadas, conforme ilustrado pelo grande número de livros e artigos publicados, mas as publicações em concreto armado e concreto protendido são, na maioria das vezes, encontrados isoladamente devido à sua heterogeneidade e anisotropia.

Segundo CONH e DINOVITZER (1994), o aço é o material mais utilizado nos estudos sobre otimização estrutural, ocupando $92 \%$ dos artigos publicados a esse respeito, enquanto o concreto e as estruturas mistas representam apenas $4 \%$ do total. Isso se deve as facilidades geradas pelo uso de um material isótropo e homogêneo, como o aço.

Na aplicação prática da otimização estrutural estão citadas estruturas do tipo: treliças planas; vigas, colunas e cabos; pórticos planos e tridimensionais, arcos; treliças espaciais; placas, cascas e chapas.

FRIEL (1974) encontrou uma solução para otimizar a taxa de armadura, de modo a minimizar o custo de vigas bi-apoiadas retangulares de concreto armado, utilizando restrições no momento aplicado, de acordo com o American Concrete Institute $(\mathrm{ACl})$.

NAAMAN (1976) comparou a otimização pelo critério de mínimo custo com a de mínimo peso para vigas bi-apoiadas protendidas de seção retangular 
e lajes armadas em uma direção. Concluiu-se que os dois critérios fornecem soluções similares apenas quando a razão entre o custo do concreto por metro cúbico e o custo da armadura para protensão por quilograma é maior que 100. Para razão menor que 100 , caso em que se encaixa a maioria das estruturas produzidas nos Estados Unidos, o critério de mínimo custo traz soluções mais econômicas.

HUANCHUN e ZHENG (1985) apresentaram um projeto de otimização de pórticos planos, baseado na norma chinesa, realizado em duas etapas: na primeira etapa buscou-se uma estrutura ótima que satisfizesse às restrições aplicadas, utilizando o método de programação linear seqüencial; e na segunda etapa, o custo do pórtico foi minimizado através da consideração das restrições locais para cada elemento, utilizando um método de busca discreta. A função custo do problema levava em consideração apenas os custos dos materiais para a execução dos pilares e vigas.

SPIRES e ARORA (1990) discutiram a otimização de estruturas em concreto armado baseada no $\mathrm{ACl}$, utilizando um procedimento de programação seqüencial quadrática. Na definição da função custo considerou-se o custo do concreto, do aço e da fôrma. Foram ainda consideradas restrições na freqüência da estrutura para limitar deslocamentos provocados pelo vento ou por terremotos.

RAMAN (1990) propôs um método não iterativo de otimização estrutural para problemas de chapas e vigas em concreto armado. Considerou-se uma variedade de funções objetivo relacionando tensão crítica, peso mínimo, deformação admissível, tensões dinâmicas, etc. para problemas estáticos e dinâmicos.

KANAGASUNDARAM e KARIHALOO (1991) apresentaram um procedimento para otimização de vigas bi-apoiadas ou contínuas e pilares que utilizava como variáveis de projeto, além das dimensões da seção transversal e da área de armadura, a resistência à compressão do concreto. Apesar da nãolinearidade das restrições utilizadas no problema, o mesmo foi resolvido por 
uma técnica matemática de programação linear, através da linearização das funções. Após a utilização de exemplos, concluiu-se que a inclusão da resistência à compressão do concreto como variável de projeto fez com que a seção otimizada fosse mais esbelta e com uma maior resistência, ocasionando também menores custos futuros de manutenção, uma vez que a utilização de concretos de resistências mais altas traz também uma melhora na durabilidade da estrutura.

CHAKRABARTY (1992) apresentou um modelo para otimização de vigas de concreto armado de seção retangular e com armadura simples, utilizando critérios de mínimo custo. Foi apresentado o modelo de formulação, indicando que o modelo pode ser resolvido através da utilização de qualquer algoritmo de programação não-linear.

HOROWITZ (1993) mostrou um método de otimização de pilares esbeltos de concreto armado considerando a maximização do momento biaxial de primeira ordem. Foi utilizado o método dos critérios de otimização, através de um algoritmo de programação seqüencial quadrática. Algumas equações de equilíbrio foram utilizadas para redução do tamanho da função, dividindo o problema em subproblemas de programação quadrática.

MOHARRAMI e GRIERSON (1993) apresentaram um método computacional para otimização de pórticos planos em concreto armado pelo critério de mínimo custo, utilizando o método de critérios de otimização. Os pilares possuíam seção transversal retangular e as vigas podiam ter seção retangular, T ou L. Foram utilizados como variáveis para minimização os custos de concreto, aço e fôrma. O arranjo das barras não foi levado em conta, sendo só utilizada como variável de projeto a área de armadura, considerando que esta estava concentrada na borda tracionada da viga e distribuída nos quatro cantos dos pilares retangulares.

SOUZA e VAZ (1993) apresentaram uma formulação para otimização do dimensionamento de um pilar padrão, com base engastada e topo livre, com seção transversal retangular em concreto armado, sujeito à flexão composta 
oblíqua. Foram utilizadas como variáveis de projeto as dimensões da seção transversal e a área total da armadura, sendo fixa a sua distribuição ao longo das faces do pilar. Como restrições, foram considerados a segurança das estruturas atendendo ao estado limite último da seção, e os valores mínimos e máximos para as variáveis de controle.

AL-SALLOUM e SIDDIQI (1994) apresentaram um procedimento de otimização para vigas de seção retangular de concreto armado, considerando o custo de concreto, aço e fôrma, utilizando as restrições do $\mathrm{ACl}$. As variáveis de otimização utilizadas foram as dimensões da seção transversal e a taxa geométrica de armadura. O processo apresentado não utiliza iterações para se chegar à solução ótima através da utilização do gradiente da função Lagrangeana e do método dos multiplicadores de Lagrange.

ADAMU e KARIHALOO (1994a) utilizaram um método de critérios otimizados tipo discretizado para minimizar os custos de uma viga de concreto armado com variação de seção transversal, usando a altura ou a altura e a taxa de armadura como variáveis de projeto. ADAMU e KARIHALOO (1994b) discutiram sobre a minimização dos custos de vigas em concreto armado com seção transversal uniforme e variação da taxa de armadura em cada vão.

Utilizando o mesmo método, ADAMU e KARIHALOO (1995a) projetaram pórticos planos baseados nas normas australianas e no Comete EuroInternational du Beton (CEB), com pilares submetidos à flexão composta normal. A função custo incluía os custos de concreto, aço e fôrma, e as variáveis de projeto eram as dimensões da seção transversal e a taxa de armadura. Por razões práticas assumiu-se que as dimensões das seções transversais permaneciam constantes para todos os trechos das vigas e dos pilares, porém variando a taxa de armadura. Em outro trabalho, ADAMU e KARIHALOO (1995b) utilizaram os pilares trabalhando à flexão composta oblíqua, ainda utilizando o pórtico plano.

ZIELINSKI et al. (1995) apresentaram um procedimento para otimizar um pilar curto de concreto armado. O procedimento incluiu duas etapas de 
iteração: na primeira etapa, foi encontrada a capacidade resistente de uma coluna com dimensões dadas e na segunda, foi realizado o processo de otimização. As variáveis de projeto foram as dimensões da seção transversal, a área de aço e o número de barras de armadura. As restrições foram baseadas em normas canadenses. A otimização foi feita utilizando a técnica de penalização interna da função, que consiste, basicamente, em transformar um problema com restrição em outro sem restrição, mediante a adição de um termo de penalização na função objetivo. Para a determinação da posição da linha neutra da seção, no caso da flexão composta oblíqua, foi utilizado o método de Newton-Raphson.

KOCER e ARORA (1996) compararam diversas técnicas de otimização para minimização de custos de uma torre de transmissão em concreto protendido, e concluíram que o método dos algoritmos genéticos foi o mais eficiente na otimização, apresentando redução de custos de aproximadamente $25 \%$ em relação ao projeto original.

FADDAE e GRIERSON (1996) apresentaram um processo de otimização com minimização de custos para pórticos espaciais em concreto armado, utilizando o método dos critérios de otimização, e restrições apropriadas para a combinação entre esforços normais, momentos fletores e esforços cortantes. Os autores concluíram que o esforço cortante é uma consideração importante para o projeto de pilares e que sua inclusão aumenta significativamente os custos da estrutura.

SHIH (1997) apresentou três técnicas que aplicam penalizações na construção de um algoritmo para solução de um problema de otimização nãolinear multiobjetiva. Foi apresentado um exemplo de otimização da função custo de uma viga em concreto armado para comprovar a eficiência do algoritmo.

KRAKOVSKI (1997) mostrou como as especificações de projeto reunidas com o princípio das variáveis divididas podem ser aplicados para a otimização de estruturas em concreto armado, exemplificada através da 
otimização da seção transversal de um reservatório de água em concreto armado. A otimização foi efetuada através de um processo iterativo. Programas de computador disponíveis para os projetos convencionais de estruturas em concreto armado puderam ser usados.

BALLING e YAO (1997) apresentaram um procedimento para otimização de pórticos espaciais retangulares de concreto armado, utilizando um método em que o número, o diâmetro e o arranjo das barras longitudinais de aço são levados em conta na otimização de vigas e pilares. Fazendo uma comparação entre este método e o método tradicional de se usar como variável apenas a área de aço da armadura, mantendo-se fixa a sua distribuição, provou-se que estes dados não influenciam na definição do custo ótimo da estrutura, de modo que podem ser retirados do processo de otimização sem prejuízo dos resultados. Baseado nestes dados foi apresentado um método simplificado, que elimina a área de aço das variáveis de projeto, deixando-a apenas como função do esforço atuante, reduzindo o tempo de processamento em relação ao método tradicional, com a mesma qualidade na otimização.

SOARES (1997) apresentou um programa para uso em prédimensionamento ótimo do vigamento de um pavimento através de um método de aproximações combinadas, no qual é feita a otimização, utilizando o método dos critérios de otimização, das seções transversais mais solicitadas de cada viga. Partiu-se do princípio que o somatório dos mínimos locais interagidos representava o mínimo global da estrutura. As variáveis envolvidas no processo de otimização foram a altura da viga e a área de aço, considerando que a largura da viga é um dado definido pela arquitetura. Através de exemplos e de comparações com estruturas reais, comprovou-se a eficiência do programa apresentado.

COELLO et al. (1997) apresentaram um método de otimização de projetos de vigas de concreto armado sujeitas à um conjunto de restrições específicas. Como há um número infinito de dimensões de vigas e taxas de armadura que produzem o mesmo momento resistente, torna-se difícil alcançar um projeto com o mínimo custo por métodos iterativos convencionais. Sendo 
assim, apresentaram um método baseado nos algoritmos genéticos. Várias aplicações mostraram como o sistema proporciona projetos mais realísticos que outros métodos baseados em técnicas de programação matemática. Também, mostraram resultados de experimentos com vários esquemas de representação para o algoritmo genético, e a metodologia que utilizaram para ajustar seus parâmetros, isto é; tamanho da população, relações de mutação e recombinação; e o número máximo de gerações produziu uma resposta razoável em um curto espaço de tempo. Um protótipo deste sistema foi correntemente testado, para verificar seu potencial de uso como uma ferramenta em aplicações reais.

Um procedimento para a pesquisa de modelos ótimos de bielas e tirantes de estruturas em concreto armado foi proposto por BIONDINI et al. (1998).

IMAM (1998) apresentou um modelo de otimização de cascas em concreto armado com espessura variável considerando o peso próprio como carregamento dominante. O modelo foi desenvolvido através do método dos elementos finitos. O critério de otimização adotado para o concreto envolveu restrições nas tensões principais em pontos críticos da estrutura e uma avaliação da resistência à compressão através de um critério de falha.

HASSANAIN e LOOV (1999) utilizaram técnicas de minimização de custos para provar as vantagens da utilização do concreto de alto desempenho para a fabricação de vigas pré-moldadas de seção I em pontes. Foi utilizado um programa computacional para fazer comparações através da variação do número de vigas utilizado, da altura da viga, dentre os padrões utilizados pelo código canadense, e do $\mathrm{f}_{\mathrm{ck}}$ do concreto utilizado.

RATH et al. (1999) utilizaram um método para variação da forma de elementos isolados de concreto armado submetidos à flexão. A partir de uma seção inicial retangular e única ao longo do comprimento, a viga passa, após a otimização, a apresentar seção I, com altura variável ao longo do comprimento. Para a otimização foram utilizadas diferentes técnicas, como programação 
quadrática para a variação de forma e algoritmo genético para determinação do número e diâmetro das barras. A otimização da peça foi feita levando-se em conta a redução no volume de concreto e assumindo-se que o custo da fôrma é proporcional a este. Desta forma, este tipo de otimização traz vantagens basicamente para peças pré-moldadas que serão produzidas em larga escala ou para grandes vigas de pontes, onde a redução do volume proporciona uma redução maior que os custos provocados pelo aumento dos cortes na fôrma.

Uma formulação por elementos finitos de placas foi desenvolvida por POULSEN et al. (2000) utilizando técnicas de programação linear. Três elementos foram estabelecidos, ou seja, elemento de placa triangular, elemento de barra e elemento de viga. O método foi aplicado para estruturas de placas em concreto armado, modelando tanto a armadura concentrada quanto a distribuída. Um eficiente esquema computacional foi usado, reduzindo o tamanho do problema.

LIANG et al. (2000) propuseram um método de otimização de estruturas contínuas sujeitas à restrições nos deslocamentos pelo uso de um novo conceito de projeto baseado no desempenho. O índice de desempenho pôde ser utilizado para comparar a eficiência de estruturas produzidas por métodos de otimização diferentes. Vários exemplos de vigas em concreto armado demonstraram a capacidade da otimização baseada no desempenho, selecionando a melhor configuração de mínimo peso de estruturas contínuas com a máxima rigidez.

Um estudo de otimização do custo de vigas e lajes em concreto armado, sujeitas à restrições nos deslocamentos e esforços, foi proposto por MATOUS et al. (2000) através da aplicação dos algoritmos genéticos.

\section{2 'Estado-da-arte' da Pesquisa em Confiabilidade}

A maioria dos projetos de engenharia são finalizados sem 0 conhecimento completo de informações; conseqüentemente, a garantia do 
desempenho pode ser, raramente, completa. Além disso, muitas decisões que são necessárias durante o processo de planejamento são feitas sob condições de incertezas. Por essa razão, há alguma chance de não satisfazer ou falhar; conseqüentemente, o risco é inevitável. Segundo FREUDENTHAL (1947), sob tais condições, não é provável (praticamente ou economicamente) assegurar absoluta segurança ou desempenho de sistemas de engenharia.

Segundo SOARES (2001), risco pode ser definido de várias formas, sua definição depende das circunstâncias. Se um evento não esperado levar a perda de vidas humanas, prejudicar o meio ambiente ou gerar algum prejuízo econômico tem-se um evento acidental. Um possível resultado de um evento acidental seria a conseqüência ou efeito.

De acordo com a definição de VATN (1998), risco é definido em relação a um evento acidental. O risco associado a um evento acidental é uma combinação de probabilidades dos eventos acidentais, ou freqüências de eventos acidentais, com a magnitude das conseqüências de ocorrências. As conseqüências representam efeitos diferentes, isto é, perda de vidas, poluição do meio ambiente, etc., conseqüentemente, o risco envolve um espectro de conseqüências, sendo assim, multidimensional. A proposta de execução de uma análise de risco é identificar eventos acidentais que podem ocorrer durante a atividade analisada, e avaliar em que extensão esses eventos podem acontecer.

Para ANG e TANG (1974), sistemas estruturais, ocasionalmente, falham na sua função pretendida, incluindo raros exemplos de colapso de grandes estruturas. Do ponto de vista da mecânica estrutural, a solicitação (S) é uma função de variáveis de deslocamentos, deformações e tensões. A resistência (R) é uma função de variáveis aleatórias caracterizando a capacidade resistente da seção ou da estrutura. O principal objetivo do projeto da mecânica estrutural é fazer com que a resistência seja superior à solicitação, ou seja, R maior que S. Em outras palavras, para saber se a estrutura pertence ao domínio de ruína ou de segurança, é necessário verificar se a 
resistência é superior à solicitação, sendo a probabilidade de ruína $p_{F}$ definida por :

$$
p_{F}=P(S \geq R)
$$

Ruína defini-se como a incapacidade do componente ou do sistema de funcionar como foi projetado e confiabilidade como a probabilidade de sobrevivência de um componente ou um sistema desde que utilizado de acordo com as especificações de projeto, ou seja, confiabilidade ( $p_{s}$ ) é dada pelo complemento da probabilidade de ruína:

$$
p_{S}=1-p_{F}
$$

Os principais objetivos da análise de confiabilidade, segundo MOHAMED (1998), são:

- fornecer um bom conhecimento das incertezas no comportamento estrutural;

- $\quad$ proporcionar um tratamento realístico das incertezas e calibrar os coeficientes parciais de segurança;

- oferecer uma real medida de segurança e, conseqüentemente, melhorar a estimativa do risco;

- o projeto pode ser melhor dimensionado e, conseqüentemente, mais econômico, possibilitando uma distribuição ótima de materiais entre os elementos estruturais;

- possibilitar a administração de um sistema existente com um custo ótimo;

- garantir que a análise de confiabilidade seja facilitada na elaboração do projeto.

LI e MELCHERS (1995) apresentaram uma técnica para calcular a probabilidade de ruptura de colunas de concreto armado sob ações combinadas de momento fletor e força axial quando as cargas aplicadas são 
estacionárias através de processos estocásticos. Obteve-se uma superfície não-linear para o estado limite da estrutura.

$\mathrm{Na}$ otimização estrutural existem incertezas e aleatoriedades nas propriedades dos materiais e nas condições de contorno. Há a necessidade de redução da probabilidade de falha estrutural através da análise estocástica, ao invés de uma aproximação determinística. LUO e GRANDHI (1996) apresentaram uma técnica usada em análises estruturais através de um programa de otimização baseado em análises de confiabilidade. A implementação e modificação da entrada das informações de natureza aleatória foram demonstradas diretamente em exemplos de treliças e estruturas expostas ao vento.

BLJUGER e YANKELEVSKI (1996) consideraram o problema de otimização de vigas em concreto armado associando a aplicação de métodos confiabilísticos para a determinação da probabilidade de ruptura. Esse método envolveu a determinação do gradiente da função do estado limite da estrutura. No problema proposto somente a avaliação numérica da função do estado limite e, conseqüentemente, o seu gradiente, foram baseados pelo método dos elementos finitos. Neste caso, foi difícil se obter uma estimativa precisa do gradiente, assim a convergência deste método não foi garantida. Deste modo, um novo método baseado em uma busca direcional foi considerado, apresentando uma alta convergência.

Uma técnica de otimização para projetos de vigas em concreto armado para pontes foi apresentada por LIN e FRANGOPOL (1996). O comportamento e as restrições especificados pelo código da American Association of State Highway and Transportation Officials (AASHTO) para pontes foram considerados no processo. Duas formulações para otimização foram apresentadas. A primeira utilizou fatores de projeto de resistência e carga (LRFD) e a segunda foi inteiramente baseada em uma aproximação confiabilística. Nestas formulações, as variáveis dependentes e independentes foram identificadas, e as restrições foram formuladas tanto no formato AASHTO-LRFD quanto no formato AASHTO-confiabilístico. Exemplos 
ilustraram a aplicação da técnica proposta para o projeto de vigas $\mathrm{T}$ em concreto armado. Análises de sensibilidade foram também feitas para encontrar os efeitos de vários parâmetros, incluindo a relação concreto-aço e o nível de confiabilidade admissível na solução ótima.

ELLINGWOOD (1996) propôs um novo critério para a consideração do carregamento e da resistência nos estados limites, baseado em métodos probabilísticos, para a avaliação da confiabilidade de estruturas já existentes através de exemplos em vigas de concreto armado.

FRANGOPOL et al. (1996) apresentaram uma investigação da confiabilidade de colunas em concreto armado. Para colunas curtas, o modelo de fibra foi usado para gerar superfícies de falha, deformações e históricos de tensões, tanto para fibras de aço quanto de concreto, sob carregamentos seqüenciais e proporcionais. Dois critérios de falha, um baseado na coleção de pontos de picos de carga e outro baseado nas deformações máximas no concreto foram apresentados. Para colunas esbeltas, superfícies de ruptura foram geradas usando um método proposto por BAZANT et al. (1991). A estimativa de confiabilidade de colunas curtas e esbeltas sob cargas aleatórias foi formulada pela simulação de Monte Carlo.

LUNDBERG e GALAMBOS (1996) realizaram testes de capacidade em perfis de aço revestidos em concreto, tubos de aço revestidos em concreto, vigas e colunas em concreto armado, e compararam os resultados com modelos de projeto do American Institute of Steel Construction (AISC). Os estudos de confiabilidade pelos momentos de primeira e segunda ordem revelaram que os elementos revestidos em concreto excederam um índice de confiabilidade alvo, mas que os tubos revestidos em concreto tiveram um índice de confiabilidade inadequado quando comparado com o valor alvo.

PU et al. (1996) apresentaram um algoritmo para a determinação do índice de confiabilidade de estruturas e aplicaram na otimização de pórticos em concreto armado. Como resultado desta formulação obteve-se uma estrutura 
$13 \%$ mais leve que uma estrutura dimensionada pelos critérios convencionais de projeto.

No trabalho de NIELSEN et a.I. (1998), discutiu-se o uso de métodos desenvolvidos para o cálculo de confiabilidade de estruturas como uma ferramenta para calcular probabilidades na análise quantitativa de risco. Para aplicar esses métodos é necessário modelar o sistema, as incertezas e os parâmetros de correlação separadamente e sistematicamente. Isto é assegurado ao modelar sistemas por combinações lógicas de funções de estado limite e modelar incertezas por fixação de distribuições de probabilidade marginal e medidas de correlação adequadas. Essas propriedades podem permitir ao analista incluir mais conhecimentos na análise, comparados a modelos tradicionalmente aplicados em análise de risco quantitativo. Portanto, a teoria da probabilidade permite que considerações subjetivas sejam incluídas na análise de risco de uma forma consistente.

Incertezas em cargas aplicadas foram apresentadas dentro da teoria de otimização pelo uso de modelos convexos elipsoidais propostos por PANTELIDE (2000). Derivações matemáticas para quantificar incertezas com modelos convexos foram apresentadas e incorporadas dentro de um algoritmo computacional de otimização. O algoritmo foi usado em uma viga contínua com dois tramos em concreto armado com variações nos níveis de incerteza. Projetos de vigas foram obtidos sob carregamento nominal empregando o modelo convexo, e sob carregamento incerto usando a condição de carregamento máximo. O custo total de estruturas ótimas foi minimizado e projetos resultantes foram comparados. As comparações mostraram que os custos de projetos ótimos produzidos sob o modelo convexo são menores do que custos de projeto sob carregamento nominal.

Uma metodologia de otimização de vigas em concreto armado baseada na confiabilidade estrutural com comportamento geometricamente não-linear foi proposta por ANTÓNIO (2001). A formulação envolveu tensões probabilísticas, e restrições de deslocamento e deformação. Na estrutura foi avaliado o índice de confiabilidade usando um método de aproximação do momento de segunda 
ordem segundo Lind-Hasofer, junto com o procedimento iterativo de NewtonRaphson. As variáveis aleatórias foram as propriedades mecânicas dos materiais tratados como materiais homogêneos e ortotrópicos. O projeto ótimo foi obtido por um algoritmo genético baseado na minimização do peso sob confiabilidades prescritas.

Uma aproximação para a resolução de projetos estruturais ótimos envolvendo termos de confiabilidade na função objetivo e no conjunto de restrições foram discutidos por ROYSET et al. (2001). Uma vantagem importante da aproximação é que a confiabilidade exigida e os cálculos de otimização são completamente desacoplados, permitindo assim uma flexibilidade na escolha do algoritmo de otimização e do método computacional da confiabilidade.

Segundo MOHAMED et al. (2001), o projeto de estruturas de concreto armado é baseado na verificação de métodos definidos por especificações padrões, onde os fatores de segurança parciais são apresentados para garantir a segurança. Um novo formato para fatores de segurança para aço e concreto foi proposto, a fim de garantir confiabilidade de objetivo uniforme. Estes fatores foram dados em termos de parâmetros de projeto de colunas, tais como resistência dos materiais, taxa de armadura, esbeltez e excentricidade do carregamento. O modelo mecânico adotado levou em conta a não-linearidade geométrica e do material. A técnica da superfície de resposta foi usada para avaliar a confiabilidade estrutural. Aplicações numéricas mostraram como a calibração de fatores de segurança conduziu a um projeto melhor do que o proposto pelo EUROCODE.

AKTAS et al. (2001) apresentaram um procedimento para a calibração dos fatores de segurança baseado na otimização da segurança e dos custos. Esse método probabilístico considera o comportamento aleatório e as incertezas quanto aos materiais e os carregamentos. O estudo foi ilustrado pela determinação dos fatores de segurança que puderam ser aplicados em uma especificação de projeto para pontes em concreto armado. 
SOARES et al. (2002) propuseram uma formulação para determinar a confiabilidade de estruturas de concreto armado, nas quais as não-linearidades física e geométrica são levadas em conta. O modelo não-linear adotado permitiu a representação do comportamento mecânico de estruturas de concreto armado no estágio de ruptura, que é governada por possíveis efeitos de grandes deslocamentos. O modelo de confiabilidade foi baseado nas superfícies de ruptura adaptáveis representando os modelos mecânicos de resposta. A superfície de ruptura foi obtida pelo ajuste das forças internas no estado último da estrutura usando um polinômio quadrático. O índice de confiabilidade foi estimado pelo algoritmo de Rackwitz e Fiessler, que tem mostrado convergir depois de um número reduzido de iterações. Uma análise numérica paramétrica de colunas e pórticos foi apresentada para aplicações práticas, onde os coeficientes de segurança parciais propostos por códigos internacionais foram associados a índices de confiabilidade.

Segundo FRANCHIN et al. (2002), o método de fator de correção modelo (MCFM) foi usado em conjunto com o método de confiabilidade de primeira ordem (FORM) para resolver problemas de confiabilidade estrutural envolvendo integrais de campos aleatórios não gaussianos. A aproximação substituiu a função de estado limite com um estado limite idealizado, no qual as integrais foram consideradas gaussianas. Análises FORM convencionais produziram a linearização de pontos de uma superfície de estado limite idealizado. Um fator de correção modelo foi então apresentado para substituir a superfície de estado limite idealizado no lugar da superfície de estado limite real. Poucas iterações produziram uma boa aproximação do índice de confiabilidade para o problema original. Este método tem aplicação para muitos problemas na engenharia civil que envolvem a aleatoriedade das propriedades dos materiais ou carregamentos.

$\mathrm{Na}$ avaliação da confiabilidade de pórticos em concreto armado, uma metodologia de superfícies de resposta foi sugerida por GUAN e MELCHERS (2002) como um modo de estimar a função do estado limite. A superfície de resposta foi construída por uma função polinomial implícita para um número de pontos. A locação destes pontos foi analisada, mas os efeitos de variação das 
suas locações têm tido pequena atenção na literatura. Foi notado que a probabilidade pode ser tanto sub quanto super estimada, dependendo da escolha dos pontos, mas não a esclarece como fazer a seleção de pontos em qualquer caso.

O envelhecimento de estruturas e programas para extensão da vida útil precisam de monitoramento contínuo para garantir a segurança de operação. Assegurando um nível máximo de risco, exige-se a definição de um programa de inspeção para estas estruturas. Poucas inspeções aumentam a probabilidade de falha. Inspeções em excesso conduzem a um aumento nos custos do tempo de vida. É difícil fazer uma estimativa do custo quando a deterioração e o comportamento estrutural são assumidos através de modelos matemáticos ou experiências anteriores. Vários métodos foram propostos por SINGH e KOENKE (2000), ESTES e FRANGOPOL (2001), GARBATOV e SOARES (2001) e NAKANISHI e NAKAYASU (2002) para minimizar os custos de reparos, resultando em diferentes estratégias de manutenção baseadas na confiabilidade.

Um estudo baseado na minimização do custo de uma estrutura durante a vida útil foi proposto por WEN (2001). As incertezas nos carregamentos e na resistência foram modeladas como variáveis aleatórias. O método foi aplicado em projetos de edifícios em concreto armado sujeitos à ação do vento e de terremotos. A questão para uma confiabilidade uniforme sob riscos diferentes foi também analisada. Concluiu-se que um projeto desenvolvido através de técnicas de otimização é viável sob vários riscos.

Um método para determinar a confiabilidade ótima foi proposto por KANDA e ADACHI (2001) com base em um conceito de custo total mínimo. Efeitos de carregamento foram considerados por análises probabilísticas. A proposta deste estudo foi identificar os efeitos de fatores probabilísticos no índice de confiabilidade ótima e no custo do ciclo de vida. Exemplos numéricos revelaram que estes fatores algumas vezes afetam significativamente a confiabilidade ótima, mas não o custo do ciclo de vida. O custo total mínimo 
forneceu um projeto com confiabilidade ótima para diferentes modelos de probabilidade de carregamentos. 


\section{OTIMIZAÇÃO MATEMÁTICA}

\subsection{Introdução}

Otimização é uma importante ferramenta na tomada de decisões e na análise de sistemas físicos. Para usá-la, deve-se primeiro identificar algum objetivo que pode ser uma quantidade ou a combinação de quantidades que podem ser representadas por um número. O objetivo depende de certas características do sistema, chamadas variáveis ou incógnitas. A meta é encontrar valores para as variáveis que otimizem o objetivo.

O processo de identificação do objetivo, das variáveis e restrições para um dado problema é conhecido como modelagem. A construção de um modelo apropriado é o primeiro passo, algumas vezes o mais importante, no processo de otimização. Se o modelo for muito simplista, não fornecerá critérios adequados dentro do problema prático, mas se for muito complexo, será muito difícil de se resolver.

Uma vez que o modelo foi formulado, um algoritmo de otimização pode ser usado para encontrar a solução. Não há um algoritmo de otimização universal. Existem numerosos algoritmos, cada qual adaptado para um tipo de problema de otimização. É de responsabilidade do usuário a escolha de um algoritmo que seja apropriado para uma aplicação específica. Esta escolha pode determinar se o problema pode ser resolvido rápido ou lentamente e se, de fato, a solução será a correta.

Depois que um algoritmo de otimização é aplicado ao modelo, pode-se identificar se houve sucesso na tarefa de encontrar a solução. Em muitos casos, há expressões matemáticas conhecidas como condições ótimas para verificar se o conjunto de variáveis é realmente a solução do problema. Se as 
condições ótimas não forem satisfeitas, deve-se fornecer informações de como a estimativa da solução poderá ser melhorada. Finalmente, o modelo poderá ser melhorado através da aplicação de técnicas que revelam a sensibilidade da solução mudar com o modelo e os dados.

\subsection{Tipos de Otimização}

\subsubsection{Otimização Discreta e Otimização Contínua}

O termo otimização discreta, usualmente, refere-se a problemas em que a solução procurada é um de um número de objetos de um conjunto finito. Contrariamente, problemas de otimização contínua encontram uma solução em um conjunto infinito, tipicamente um conjunto de vetores com componentes reais.

\subsubsection{Otimização Restrita e Otimização Não-Restrita}

Problemas de otimização não-restrita resultam diretamente em muitas aplicações práticas. Se há restrições naturais nas variáveis, algumas vezes, é mais seguro desconsiderá-las e assumir que as variáveis não têm efeito na solução ótima. Problemas não-restritos surgem também como reformulações de problemas de otimização restrita, em que as restrições são substituídas por termos de penalização na função objetivo.

Quando, simultaneamente, a função objetivo e todas as restrições são funções lineares de x, o problema é de programação linear. Caso contrário, tem-se um problema de programação não-linear. 


\subsubsection{Otimização Local e Otimização Global}

Os mais rápidos algoritmos de otimização procuram somente uma solução local, um ponto em que a função objetivo é a menor que para os outros pontos possíveis em uma vizinhança. Estes algoritmos não encontram a menor de todas as mínimas, que é a solução global. Soluções globais são necessárias em algumas aplicações, mas geralmente são difíceis de identificá-las.

\subsubsection{Otimização Estocástica}

Em alguns problemas de otimização, o modelo não pode ser completamente especificado porque depende de quantidades que são desconhecidas na hora da formulação. Portanto, modeladores podem predizer ou estimar as quantidades desconhecidas com alguns graus de confiança. Algoritmos de otimização estocástica usam quantificações de incertezas para produzir soluções que otimizem o desempenho esperado do modelo.

\subsection{Formulação Matemática de Problemas de Otimização}

Otimização matemática é a minimização ou maximização de uma função sujeita ou não à restrições em suas variáveis. Usa-se a seguinte notação:

- $\quad$ X é o vetor das variáveis, também chamado incógnitas ou parâmetros;

- $\quad f$ é a função objetivo. É uma função de $x$ que se deseja maximizar ou minimizar;

- $\quad$ c é o vetor das restrições que as incógnitas devem satisfazer. É um vetor função das variáveis $x$. O número de componentes em c é o número de restrições das variáveis. 
O problema de otimização pode ser escrito como:

$$
\min _{x \in R^{n}} f(x), \quad \text { sujeito à }\left\{\begin{array}{l}
g_{i}(x) \geq 0 \\
h_{j}(x)=0
\end{array}\right.
$$

Onde $f, g_{i}$ e $h_{j}$ são funções escalares estimadas das variáveis $x$.

A Figura 3.1 mostra o contorno da função objetivo, isto é, o conjunto de pontos para que $f(x)$ tenha um valor constante. Também ilustra a região provável, que é o conjunto de pontos que satisfazem todas as restrições, e o ponto ótimo $x^{*}$, a solução do problema. O "lado improvável" das restrições de desigualdade é o hachurado.

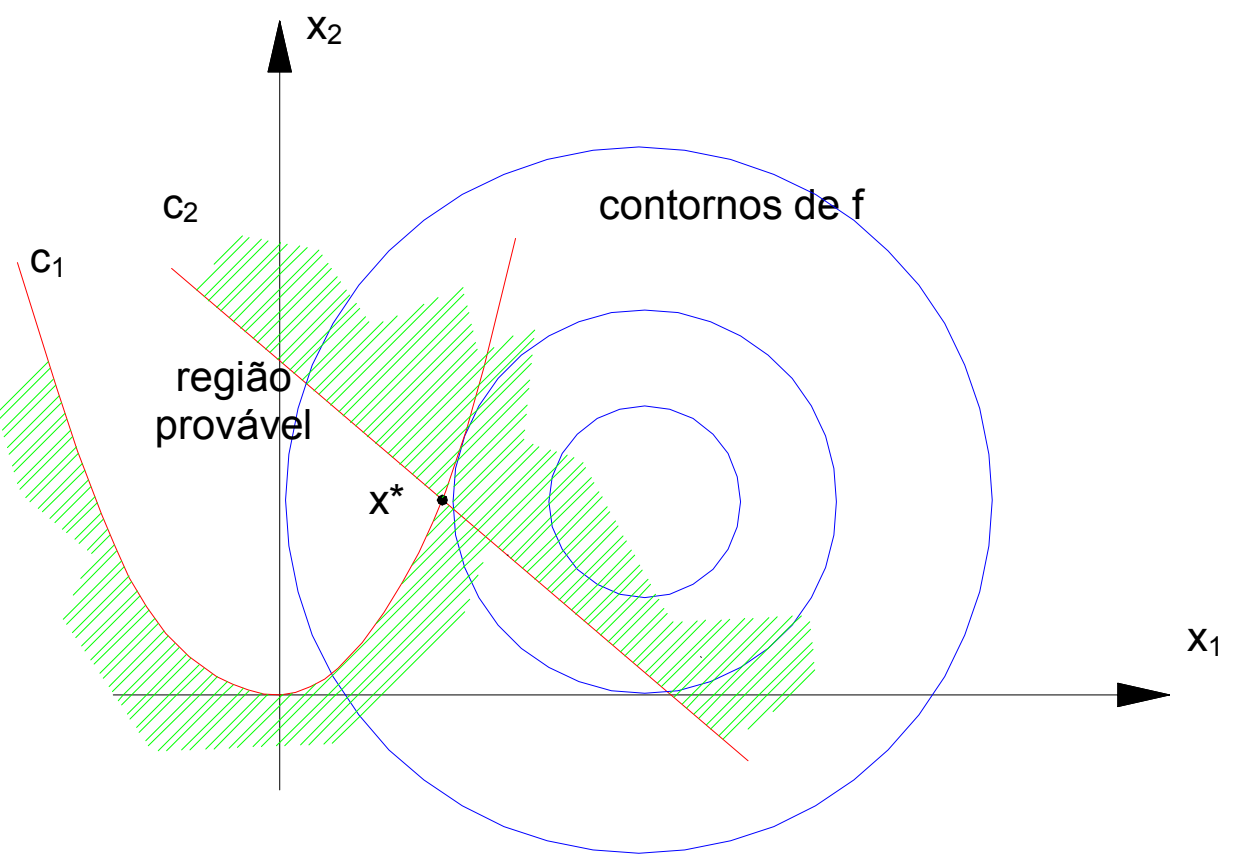

Figura 3.1 - Representação geométrica de um problema de otimização

\subsection{Algoritmos de Otimização}

Os algoritmos de otimização são iterativos. Começam com uma estimativa inicial dos valores ótimos e geram uma seqüência de estimativas refinadas até encontrar a solução. A estratégia usada para mover uma iteração 
para a seguinte distingue um algoritmo de outro. A maioria das estratégias faz uso de valores da função objetivo, das restrições, e da primeira e da segunda derivadas destas funções. Alguns algoritmos acumulam informações colecionadas em iterações anteriores, enquanto outros usam somente informações locais do presente ponto. Independente destas características, todos os bons algoritmos devem possuir as seguintes propriedades:

- Robustez: bom desempenho em uma ampla variedade de problemas, para todas as escolhas das variáveis iniciais.

- Eficiência: não devem exigir muito tempo de computador e memória.

- $\quad$ Acurácia: devem ser capazes de identificar uma solução com precisão, sem estar excessivamente sensível a erros nos dados ou para erros de arredondamentos aritméticos que ocorram quando o algoritmo está sendo implementado em um computador.

Estes objetivos podem ser conflitantes. Por exemplo, um método rápido de convergência para programação não-linear pode precisar de muita memória do computador para grandes problemas. De outro modo, um método robusto pode também ser o mais lento.

Para ilustrar a idéia, considera-se o problema para encontrar um valor mínimo y $^{*}$ para a função:

$$
y=f(x), y \in R^{1}, \quad x \in R^{n}
$$

Começa-se a pesquisa no ponto de início $x^{(1)} \in R^{n}, y^{(1)}=f\left\{x^{(1)}\right\} \neq y^{*}$, opera-se neste ponto inicial e de acordo com alguns critérios chega-se em um novo ponto $x^{(2)}, y^{(2)}=f\left\{x^{(2)}\right\}<y^{(1)}$. Pode-se utilizar os critérios usados anteriormente para generalizar um novo ponto pela definição de uma função de pesquisa $A$ que leva qualquer ponto de $R^{n}$ a outro ponto de $R^{n} e$, em particular, $x^{(1)} a x^{(2)}$, isto é, $x^{(2)}=A\left\{x^{(1)}\right\}$. No segundo estágio ou iteração da pesquisa, usa-se $x^{(2)}, y^{(2)}$ como ponto inicial e chega-se por meio dos mesmos critérios 
em $x^{(3)}=A\left\{x^{(2)}\right\} \in R^{n}, y^{(3)}=f\left\{x^{(3)}\right\}<y^{(2)}$. A pesquisa continua desta maneira com a k-ésima iteração. Deste modo, tem-se

$$
x^{(k+1)}=A\left\{x^{(k)}\right\}, \quad y^{(k+1)}=f\left\{x^{(k+1)}\right\}
$$

Este procedimento gera uma seqüência de pontos $\mathrm{x}^{(\mathrm{k})}$; na maioria dos casos práticos, esta seqüência é infinita e pode, conseqüentemente, ser escrita $\{x(k)\}_{1}^{\infty}$. Para o procedimento fornecer a resolução do problema de minimização, é necessário que

$$
x^{(\infty)}=x^{*}
$$

onde

$$
f\left(x^{*}\right)=y^{*}
$$

$\mathrm{Na}$ resolução do atual problema, não se pode executar um número infinito de operações necessárias para atingir $x^{(\infty)}$, então se deve terminar a pesquisa depois de um número finito $\mathrm{k}$ de iterações, escolhendo-se $\mathrm{k}$ de modo a alcançar a acurácia exigida. Esta acurácia pode ser expressa na forma

$$
\left|y^{(k+1)}-y^{*}\right| \leq \Delta^{\prime}
$$

onde $\Delta^{\prime}$ é um número positivo tão pequeno quanto se deseja; desde que $\mathrm{y}^{\star}$ seja conhecido, deve-se determinar k por algum outro critério para o término do processo, ou seja,

$$
\left|\nabla f\left\{x^{(k+1)}\right\}\right| \leq \Delta ”
$$

Existem vários tipos de critérios que podem ser usados para decidir quando o procedimento iterativo gera uma aproximação apropriada para o ponto de otimização, a maioria destes podem ser escritos pela definição de uma função $B: R^{n} \rightarrow R^{1}$ e de um número positivo $\Delta$, tal que, se 


$$
\mathrm{B}\left\{\mathrm{x}^{(\mathrm{k}+1)}\right\} \leq \Delta
$$

então $\mathrm{x}^{(\mathrm{k}+1)}$ é suficientemente próximo de $\mathrm{x}^{*}$.

Pode-se resumir o processo, como se mostra a seguir:

Passo $1 \quad$ Escolher um ponto inicial $x^{(1)} \in R^{n}, y^{(1)}=f\left\{x^{(1)}\right\}$ e escolher um valor positivo para $\Delta$.

Passo $2 \quad$ Fazer $\mathrm{k}=0$.

Passo $3 \quad$ Fazer $k=k+1$.

Passo $4 \quad$ Fazer $x^{(k+1)}=A\left\{x^{(k)}\right\}, y^{(k+1)}=f\left\{x^{(k)}\right\}<y^{(k)}$.

Passo 5 Se $B\left\{x^{(\mathrm{k}+1)}\right\} \leq \Delta$, ir para o Passo 6; caso contrário retornar ao Passo 3.

Passo $6 \quad$ Quando $x^{*}=x^{(k+1)}, y^{*}=y^{(k+1)}$, parar.

Em muitos algoritmos, a função de pesquisa do Passo 4 é definida em termos de uma direção de pesquisa $s^{(k)}$ e um passo de extensão $r_{k}$. Assim, primeiro decide-se que $A\left\{x^{(k)}\right\}$ deve ser da forma

$$
A\left\{x^{(k)}\right\}=x^{(k)}+r s^{(k)},
$$

onde $\mathrm{s}^{(\mathrm{k})} \in \mathrm{R}^{\mathrm{n}}$ é um vetor fixo, dependente em $\mathrm{x}^{(\mathrm{k})}$, e $\mathrm{r}$ um número real desconhecido, então se escolhe um valor específico $r_{k}$ para $r$. A definição de um algoritmo exige que a duração do passo seja escolhida e que $r=r_{k}$ minimize $f\left\{x^{(k)}+r s^{(k)}\right\}$; portanto, deve-se reconhecer que, em geral, isto exigiria um número infinito de operações e o melhor a fazer na prática seria minimizar $f\left\{x^{(k)}+r s^{(k)}\right\}$ dentro dos limites de alguma acurácia especificada. 


\subsubsection{Eficiência dos Algoritmos}

Um algoritmo converge de um dado ponto inicial $x^{(1)}$ se a seqüência $\left\{x^{(k)}\right\}$ de pontos gerados tiver em seu limite a solução $x^{*}$ do problema a ser resolvido. O conjunto de pontos iniciais, dos quais um dado algoritmo converge para a solução do problema, é o seu domínio de convergência.

Seja $\mathrm{e}^{(\mathrm{k})} \mathrm{o}$ erro $\mathrm{x}^{*}-\mathrm{x}^{(\mathrm{k})}$ na k-ésima aproximação iterativa para $\mathrm{x}^{*}$ gerado pelo algoritmo. Se existir números reais $p \geq 1$ e $K \neq 0$, tal que a seqüência $\left\{\left|e^{(k+1)}\right| /\left|e^{(k)}\right| p\right\}$ converge para $K$, então $p$ será a ordem de convergência; se $p$ $>1$, a convergência será superlinear. Algoritmos com uma ordem de convergência 2 são algumas vezes denominados quadraticamente convergentes; entretanto, deve-se reservar este termo para descrever algoritmos que podem locar exatamente o mínimo da função quadrática em um número finitos de iterações.

Do ponto de vista da velocidade de convergência, algoritmos com grandes valores de $\mathrm{p}$ quando associados com grandes valores de $\mathrm{K}$ são os preferidos. Entretanto, deve-se indicar que uma ordem mais alta de convergência é associada a um menor domínio de convergência.

Outro fator importante na determinação do valor prático de um algoritmo é o trabalho necessário para cada iteração. Pode-se expressar isto, por exemplo, com o número $N_{k}$ de vezes que se deve avaliar a função $f(x)$, já que este é o principal tempo de operação. Se $\mathrm{N}_{\mathrm{k}}=\mathrm{N}$ for independente de $\mathrm{k}$, como é geralmente o caso, então a eficiência do algoritmo será definida como $\mathrm{N}^{-1} \log \mathrm{p}$; sob suposições adequadas, pode ser mostrado que a eficiência mais alta de um algoritmo implica em menos trabalho para alcançar uma solução de uma dada acurácia. 


\subsection{Fundamentos Matemáticos para Otimização}

\subsubsection{Matriz Hessiana}

Na ausência de equações de restrições, uma função objetivo $f\left(x_{1}, x_{2}, \ldots\right.$, $x_{n}$ ) contínua e diferenciável com $n$ variáveis alcança um valor máximo ou mínimo no interior de um espaço de projeto $R^{n}$ quando as $n$ derivadas parciais,

$$
\frac{\partial f}{\partial x_{1}}, \frac{\partial f}{\partial x_{2}}, \ldots, \frac{\partial f}{\partial x_{n}},
$$

são simultaneamente anuladas, condições necessárias para o ponto $x^{*}$ ser um ponto estacionário. Para uma função escalar, o vetor das derivadas primeiras, referido como o vetor gradiente $\nabla f$ é usado para encontrar direções de pesquisa em algoritmos de otimização.

O desenvolvimento de uma condição suficiente para um ponto estacionário $x^{*}$ ser um ponto de extremo exige uma avaliação da matriz das derivadas segundas $\mathrm{H}$ da função objetivo. A matriz das derivadas segundas é também chamada matriz Hessiana definida como:

$$
H=\left[\begin{array}{cccc}
\frac{\partial^{2} f}{\partial x_{1}^{2}} & \frac{\partial^{2} f}{\partial x_{1} \partial x_{2}} & \cdots & \frac{\partial^{2} f}{\partial x_{1} \partial x_{n}} \\
\vdots & \vdots & \vdots & \vdots \\
\vdots & \vdots & \vdots & \vdots \\
\frac{\partial^{2} f}{\partial x_{n} \partial x_{1}} & \frac{\partial^{2} f}{\partial x_{n} \partial x_{2}} & \cdots & \frac{\partial^{2} f}{\partial x_{n}^{2}}
\end{array}\right]
$$

Se a matriz das derivadas segundas avaliadas em $x^{*}$ for positiva definida, então o ponto estacionário será mínimo; se for negativa definida, então o ponto estacionário será um ponto máximo. Uma matriz simétrica H será dita positiva negativa definida se a forma quadrática $Q=x^{\top} H x$ for positiva (negativa) para todo $x$, e igual a zero se e somente se $x=0$. 
Uma verificação computacional para positivo e negativo na determinação da matriz envolve determinantes da menor principal, $H_{i}(i=1, \ldots, n)$. Uma menor principal $\mathrm{H}_{\mathrm{i}}$ é uma submatriz quadrada de $\mathrm{H}$ de ordem i cuja diagonal principal permanece ao longo da diagonal principal da matriz $\mathrm{H}$.

A matriz $\mathrm{H}$ será positiva definida se os determinantes de todas as principais menores locadas no canto superior esquerdo da matriz forem positivas; e negativas-definidas se $-\mathrm{H}$ for positiva definida. Alternativamente, $\mathrm{H}$ será positiva definida se $\mathrm{H}_{1}$ for negativa e seguindo as principais menores, $\mathrm{H}_{2}, \mathrm{H}_{3}, \ldots, \mathrm{H}_{\mathrm{n}}$, serão alternativamente positiva e negativa.

Uma matriz simétrica $\mathrm{H}$ será chamada positiva semidefinida se a forma quadrática $Q=x^{\top} H x$ for não-negativa para todo $x$. Isto acontece quando os autovalores da matriz são não-negativos. Infelizmente, a condição de que as menores principais são não-negativas não é uma condição suficiente. Se a matriz for positiva semidefinida, mas não-positiva definida, existe pelo menos um $x \neq 0$, tal que a forma quadrática é zero, pelo menos uma das menores principais é zero, a matriz será singular, e pelo menos um dos autovalores é zero. No caso de derivadas com ordens mais altas da função f, é necessário estabelecer condições suficientes para o mínimo.

Similarmente, quando - $\mathrm{H}$ for positiva semidefinida, então $\mathrm{H}$ será negativa semidefinida. Se $\mathrm{H}$ for negativa semidefinida, mas não-negativa definida, derivadas de ordens mais altas serão necessárias para estabelecer condições suficientes para o máximo. Finalmente, quando $\mathrm{H}$ não for positiva semidefinida nem negativa semidefinida, será chamada indefinida.

\subsubsection{Convexidade}

O conceito de convexidade é fundamental em otimização, implica que o problema é favorável em vários aspectos. O termo convexo pode ser aplicado tanto para conjuntos quanto para funções. 
$S \in R^{n}$ será um conjunto convexo se o segmento de reta ligando dois pontos quaisquer em $S$ permanecer inteiramente dentro de $S$. Formalmente, para dois pontos quaisquer $\mathrm{x} \in \mathrm{S}$ e $\mathrm{y} \in \mathrm{S}$, tem-se:

$$
\alpha \mathrm{x}+(1-\alpha) \mathrm{y} \in \mathrm{S}, \quad \text { para todo } \alpha \in[0,1]
$$

f será uma função convexa se o seu domínio for um conjunto convexo e se para dois pontos quaisquer $x$ e y neste domínio, o gráfico de $f$ permanecer sob a reta ligando $(x, f(x))$ para $(y, f(y))$ no espaço $R^{n+1}$. Isto é,

$$
f(\alpha x+(1-\alpha) y) \leq \alpha f(x)+(1-\alpha) f(y), \quad \text { para todo } \alpha \in[0,1]
$$

Uma função $f$ será dita côncava se $-f$ for convexa. As Figuras 3.2 e 3.3 ilustram a definição de conjunto convexo.

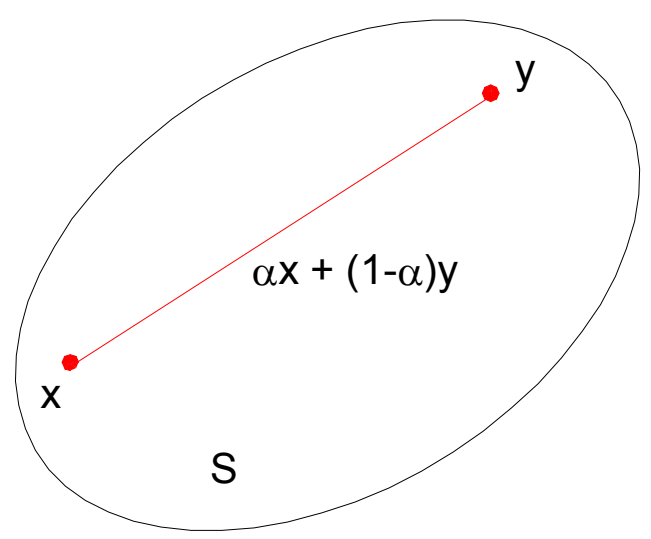

Figura 3.2 - Exemplo de um conjunto convexo 


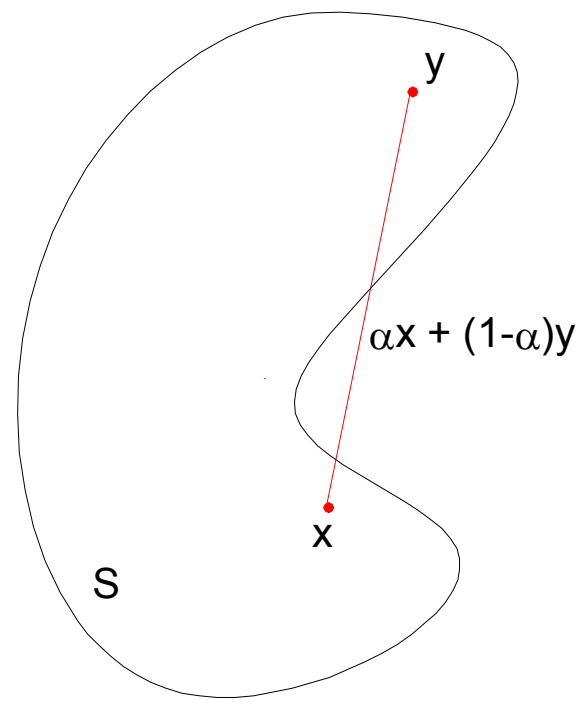

Figura 3.3 - Exemplo de um conjunto não convexo

Algoritmos de otimização sem restrições são, usualmente, utilizados para convergir para um ponto estacionário (máximo, mínimo ou ponto de inflexão) da função objetivo f. Conhecendo-se se f é convexo, então se pode estar certo de que esse ponto é um mínimo global. O termo "programação convexa" é usado para descrever um caso especial do problema de otimização restrita em que:

- $\quad$ a função objetivo é convexa;

- $\quad$ as funções de restrições de desigualdade $g_{i}(x)$ são côncavas;

- $\quad$ as funções de restrições de igualdade $h_{j}(x)$ são lineares.

A união de dois conjuntos convexos pode ser um conjunto convexo ou não-convexo, mas a interseção de dois ou mais conjuntos convexos é sempre um conjunto convexo, conforme mostrado nas Figuras 3.4 e 3.5, respectivamente. 


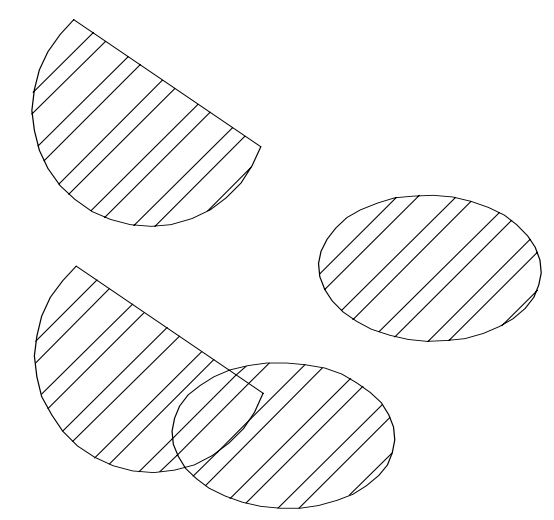

Figura 3.4 - Exemplo de união de conjuntos convexos

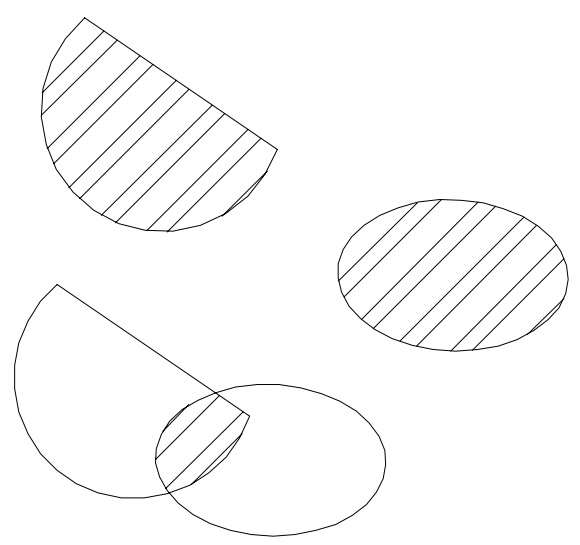

Figura 3.5 - Exemplo de interseção de conjuntos não-convexos

\subsection{Propriedades dos Pontos de Mínimo}

\subsubsection{Ponto de Mínimo Global}

Geralmente, busca-se um minimizador global de f, isto é, um ponto onde a função alcança seu menor valor em todo o domínio. Deste modo, pode-se dizer que um ponto $x^{*}$ é um minimizador global se $f\left(x^{*}\right) \leq f(x)$ para todo $x$, onde $\mathrm{x}$ varia para todo $\mathrm{R}^{\mathrm{n}}$. 


\subsubsection{Ponto de Mínimo Local}

Um minimizador local é um ponto que atinge o menor valor de $f$ na sua vizinhança. Formalmente, diz-se que um ponto $x^{*}$ é minimizador local se houver uma vizinhança $\mathcal{N}$ de $\mathrm{x}^{*}$ tal que $\mathrm{f}\left(\mathrm{x}^{*}\right) \leq \mathrm{f}(\mathrm{x})$ para todo $\mathrm{x} \in \mathcal{N}$, onde a vizinhança $\mathcal{N}$ de $\mathrm{x}^{*}$ é simplesmente um conjunto aberto que contém $\mathrm{x}^{*}$.

Um ponto que satisfaz esta definição é algumas vezes chamado minimizador local frágil. Esta terminologia distingue-o de um minimizador local rigoroso, também chamado minimizador local forte, que é um ponto $x^{*}$ em uma vizinhança $\mathcal{N}$ tal que $\mathrm{f}\left(\mathrm{x}^{*}\right)<\mathrm{f}(\mathrm{x})$ para todo $\mathrm{x} \in \mathcal{N}$ com $\mathrm{x} \neq \mathrm{x}^{*}$.

Um ponto $\mathrm{x}^{*}$ é um minimizador isolado se houver uma vizinhança $\mathcal{N}$ tal que $\mathrm{x}^{*}$ seja o único minimizador local em $\mathcal{N}$. Mas alguns minimizadores locais rigorosos podem não ser isolados, é verdade também que todos os minimizadores locais isolados são rigorosos.

A Figura 3.6 mostra uma função de uma variável que, na ausência de qualquer restrição, tem dois mínimos locais, além do seu mínimo global; esta função também tem dois máximos locais $e$, se for assumido que $f(x)$ cresce sem limite para valores positivos e negativos de $\mathrm{x}$, a função não terá máximo global. 


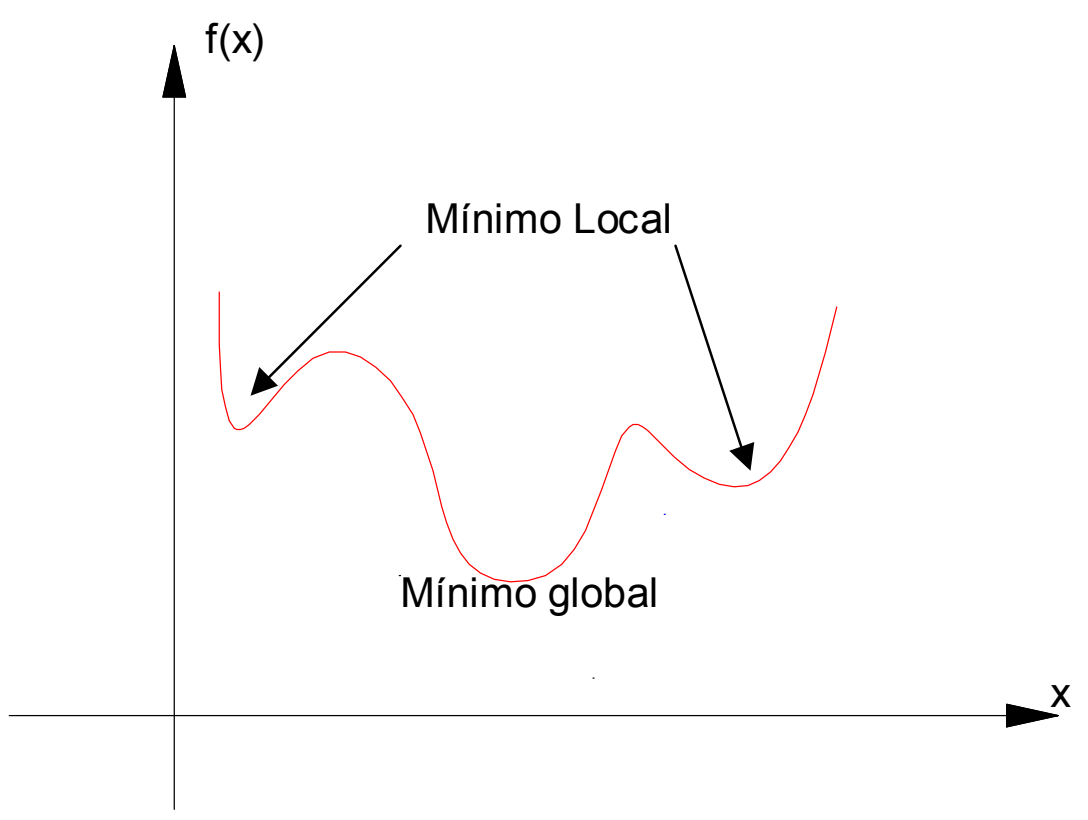

Figura 3.6 - Exemplo de uma função com um mínimo global e dois mínimos locais

Apesar dos exemplos de engenharia, raramente, gerarem problemas sem restrições, casos sem restrições proporcionam uma teoria básica que se pode desenvolver para tratar de problemas restritos.

Das definições dadas acima, pode-se parecer que o único modo de descobrir se um ponto $x^{*}$ é um mínimo local é examinar todos os pontos em sua vizinhança imediata e fazer com que nenhum deles tenha um menor valor de função.

Quando a função $f$ for plana, haverá outros modos mais eficientes e práticos para identificar o mínimo local. Em particular, se f for contínua e duas vezes diferenciável, pode-se ser capaz de dizer que $x^{*}$ será um minimizador local, e possivelmente um minimizador local rigoroso, pela análise do gradiente $\nabla \mathrm{f}\left(\mathrm{x}^{*}\right)$ e do Hessiano $\nabla^{2} \mathrm{f}\left(\mathrm{x}^{*}\right)$.

A ferramenta matemática usada para estudar os minimizadores de funções contínuas é o Teorema de Taylor. 
Teorema 1 (Teorema de Taylor).

Seja $f: R^{n} \rightarrow R$ contínua e diferenciável, e que $p \in R^{n}$. Então se tem:

$$
f(x+p)=f(x)+\nabla f(x+t p)^{\top} p
$$

para algum $t \in(0,1)$. Além disso, se f for contínua e duas vezes diferenciável, tem-se:

$$
\nabla f(x+p)=\nabla f(x)+\int_{0}^{1} \nabla^{2} f(x+t p) p d t
$$

e

$$
\nabla f(x+p)=f(x)+\nabla f(x)^{\top} p+\frac{1}{2} p^{\top} \nabla^{2} f(x+t p) p
$$

para algum $t \in(0,1)$.

As condições necessárias para a otimização são derivadas da suposição de que $x^{*}$ é um mínimo local da análise de $\nabla f\left(x^{*}\right)$ e $\nabla^{2} f\left(x^{*}\right)$.

Teorema 2 ( Condições necessárias de primeira ordem)

Se $x^{*}$ for um minimizador local e $f$ contínua e diferenciável em uma vizinhança aberta de $x^{*}$, então $\nabla f\left(x^{*}\right)=0$.

\section{PROVA}

Supõe-se por contradição que $\nabla f\left(x^{*}\right) \neq 0$. Define-se o vetor $p=-\nabla f\left(x^{*}\right)$ e nota-se que $\mathrm{p}^{\top} \nabla \mathrm{f}\left(\mathrm{x}^{*}\right)=-\left\|\nabla \mathrm{f}\left(\mathrm{x}^{*}\right)\right\|^{2}<0$. Por $\nabla \mathrm{f}$ ser contínua próxima de $\mathrm{x}^{*}$, há um escalar $\mathrm{T}>0$, tal que:

$$
\mathrm{p}^{\top} \nabla \mathrm{f}\left(\mathrm{x}^{*}+\mathrm{tp}\right)<0, \quad \text { para todo } \mathrm{t} \in[0, \mathrm{~T}]
$$


Para qualquer $\overline{\mathrm{t}} \in(0, \mathrm{~T}]$, tem-se pelo Teorema de Taylor que

$$
f\left(x^{*}+\overline{t p}\right)=f\left(x^{*}\right)+\overline{t p}^{\top} \nabla f\left(x^{*}+t p\right), \quad \text { para todo } t \in(0, \bar{t})
$$

Conseqüentemente, $f\left(x^{*}+\overline{t p}\right)<f\left(x^{*}\right)$, para todo $\bar{t} \in(0, T]$. Tem-se que na direção de $x^{*}$, f é decrescente. Então $x^{*}$ não é um minimizador local e se tem uma contradição.

Assim, $\mathrm{x}^{*}$ é um ponto estacionário se $\nabla \mathrm{f}\left(\mathrm{x}^{*}\right)=0$. De acordo com 0 Teorema 2, qualquer minimizador local deve ser um ponto estacionário.

Teorema 3 (Condições necessárias de segunda ordem)

Se $x^{*}$ for um minimizador local de $f$ e $\nabla^{2} f$ contínua em uma vizinhança aberta de $x^{*}$, então $\nabla f\left(x^{*}\right)=0$ e $\nabla^{2} f\left(x^{*}\right)$ será positivo semidefinido.

\section{PROVA}

Sabe-se do Teorema 2 que $\nabla f\left(x^{*}\right)=0$. Por contradição, assume-se que $\nabla^{2} f\left(x^{*}\right)$ não é positivo semidefinido. Assim, pode-se escolher um vetor $p$, tal que $p^{\top} \nabla^{2} f\left(x^{*}\right) p<0$, e como $\nabla^{2} f$ é contínuo próximo de $x^{*}$, há um escalar $T>0$, tal que $p^{\top} \nabla^{2} f\left(x^{*}+t p\right) p<0$, para todo $t \in[0, T]$.

Fazendo uma expansão pela série de Taylor ao redor de $x^{*}$, tem-se para todo $\overline{\mathrm{t}} \in(0, \mathrm{~T}]$ e algum $\mathrm{t} \in(0, \overline{\mathrm{t}})$ :

$$
\nabla f\left(x^{*}+\overline{t p}\right)=f\left(x^{*}\right)+\overline{t p}^{\top} \nabla f\left(x^{*}\right)+\frac{1}{2} \bar{t}^{2} p^{\top} \nabla^{2} f\left(x^{*}+t p\right) p<f\left(x^{*}\right)
$$

Como no Teorema 2, pode-se encontrar uma direção de $x^{*}$, em que f é decrescente, e novamente $x^{*}$ não é um minimizador local. 
Agora, pode-se descrever condições suficientes, que são condições nas derivadas de f no ponto $z^{*}$, que garantem que $x^{*}$ seja um minimizador local.

\section{Teorema 4}

Seja $\nabla^{2} f$ contínua em uma vizinhança aberta de $x^{*}, \nabla f\left(x^{*}\right)=0$ e $\nabla^{2} f\left(x^{*}\right)$ positivo definido. Portanto, $x^{*}$ é um minimizador local rigoroso de $f$.

\section{PROVA}

Como o Hessiano é contínuo e positivo definido em $x^{*}$, pode-se escolher um raio $r>0$, tal que $\nabla^{2} f(x)$ permanece positivo definido para todo $x$, em uma esfera $\mathscr{D}=\left\{z\left\|z-x^{*}\right\|<r\right\}$. Tomando-se qualquer vetor não nulo $p$ com $\|p\|<r$, tem-se $x^{*}+p \in \mathscr{D}$, então

$$
f\left(x^{*}+p\right)=f\left(x^{*}\right)+p^{\top} \nabla f\left(x^{*}\right)+\frac{1}{2} p^{\top} \nabla^{2} f(z) p=f\left(x^{*}\right)+\frac{1}{2} p^{\top} \nabla^{2} f(z) p
$$

onde $z=x^{*}+t p$, para qualquer $t \in(0,1)$. Desde que $z \in \mathscr{D}$, tem-se $p^{\top} \nabla^{2} f(z)>0$ e, conseqüentemente, $f\left(x^{*}+p\right)>f\left(x^{*}\right)$.

Nota-se que as condições suficientes de segunda ordem do Teorema 4 garantem algo mais forte que as condições necessárias discutidas anteriormente; nominalmente, o minimizador é um minimizador local rigoroso. Observa-se também que as condições suficientes de segunda ordem não são necessárias, um ponto $x^{*}$ pode ser um minimizador local rigoroso e ainda pode falhar para satisfazer as condições suficientes.

\section{Teorema 5}

Quando $f$ for convexa, qualquer minimizador local $x^{*}$ será um minimizador global de $f$. Se, além do mais, f for diferenciável, então qualquer ponto estacionário $x^{*}$ será um minimizador global de $f$. 


\section{PROVA}

Seja $x^{*}$ um minimizador local, mas não um minimizador global. Portanto, pode-se encontrar um ponto $z \in R^{n} \operatorname{com} f(z)<f\left(x^{*}\right)$. Considera-se um segmento de reta que liga $x^{*}$ a $z$ :

$$
x=\eta z+(1-\eta) x^{*}, \quad \text { para qualquer } \eta \in(0,1]
$$

Pela propriedade da convexidade de $\mathrm{f}$, tem-se:

$$
f(x) \leq \eta f(x)+(1-\eta) f\left(x^{*}\right)<f\left(x^{*}\right)
$$

Qualquer vizinhança $\mathcal{N}$ de $\mathrm{x}^{*}$ contém uma parte do segmento de reta (3.1), então neste trecho sempre estarão os pontos $x \in \mathcal{N}$, na qual (3.2) é satisfeita. Por essa razão, $x^{*}$ não será um minimizador local.

Para a segunda parte do Teorema, supõe-se que $x^{*}$ não é um minimizador global e tem-se z como anteriormente. Portanto, da convexidade, tem-se:

$$
\begin{gathered}
\nabla f\left(x^{*}\right)^{\top}\left(z-x^{*}\right)=\left.\frac{d}{d \eta} f\left(x^{*}+\eta\left(z-x^{*}\right)\right)\right|_{\eta=0}=\lim _{\eta \rightarrow 0} \frac{f\left(x^{*}+\eta\left(z-x^{*}\right)\right)-f\left(x^{*}\right)}{\eta} \\
\leq \lim _{x \rightarrow 0} \frac{\eta f(z)+(1-\eta) f\left(x^{*}\right)-f\left(x^{*}\right)}{\eta}=f(z)-f\left(x^{*}\right)<0 .
\end{gathered}
$$

Conseqüentemente, $\nabla \mathrm{f}\left(\mathrm{x}^{*}\right) \neq 0$, então $\mathrm{x}^{*}$ não será um ponto estacionário. 


\subsection{Fundamentos da Otimização Não-Restrita}

Em otimização sem restrições, minimiza-se uma função objetivo que depende de variáveis reais, com nenhuma restrição em todos os valores destas variáveis. A formulação matemática é:

$$
\min _{x} f(x)
$$

onde $x \in R^{n}$ é um vetor real com $n \geq 1$ componentes e $f: R^{n} \rightarrow R$ é uma função plana.

Geralmente, necessita-se uma perspectiva global na função $f$. Conhecendo-se os valores de $f$ e talvez de algumas de suas derivadas em um conjunto de pontos $\mathrm{x}_{0}, \mathrm{x}_{1}, \mathrm{x}_{2}, \ldots$, os algoritmos conseguem escolher estes pontos e identificar uma solução segura sem usar muita memória e tempo no computador.

\subsection{Fundamentos da Otimização Restrita}

A maioria dos problemas de otimização restrita pode ser colocada como:

$$
\begin{aligned}
\text { minimize } f(x), & \\
\text { sujeito à } g_{i}(x) \geq 0, & \text { para } i=1,2, \ldots, m \\
h_{j}(x)=0, & \text { para } j=1,2, \ldots, p
\end{aligned}
$$

onde $f(x), g_{i}(x)$ e $h_{j}(x)$ são funções reais do vetor $\bar{x}=\left(x_{1}, x_{2}, \ldots, x_{n}\right) \in R^{n}$.

As relações $g(x) \geq 0$ e $h(x)=0$ são chamadas, respectivamente, restrições de desigualdade e restrições de igualdade. Deve-se assumir que o objetivo e as funções de restrição são duas vezes diferenciáveis em $R^{n}$; qualquer caso onde isto não ocorra exigirá tratamento especial, geralmente, 
apontado na demonstração de que as derivadas existem onde elas forem necessárias.

\subsubsection{Restrições Ativas e Inativas}

Quando se tem uma restrição de desigualdade $g_{i}(x) \leq 0$, diz-se que essa é ativa em um ponto $x$, se $g_{i}(x)=0$ e inativa, se $g_{i}(x)<0$. Considera-se, por convenção, que qualquer restrição de igualdade $h_{i}(x)=0$ é ativa em qualquer ponto provável.

Dado um ponto provável $x$, as restrições inativas nesse ponto se comportam como restrições de igualdade. Além disso, as restrições inativas não influenciam nas restrições do problema. Por essa razão, pode-se considerar em cada ponto somente as restrições nele ativas e após encontrar o ponto ótimo, verificar se a solução encontrada satisfaz a todas as restrições.

Este fato pode ser ilustrado pela Figura 3.7, onde as propriedades locais satisfeitas por $x^{*}$, obviamente, independem das restrições inativas $g_{2}$ e $g_{3}$.

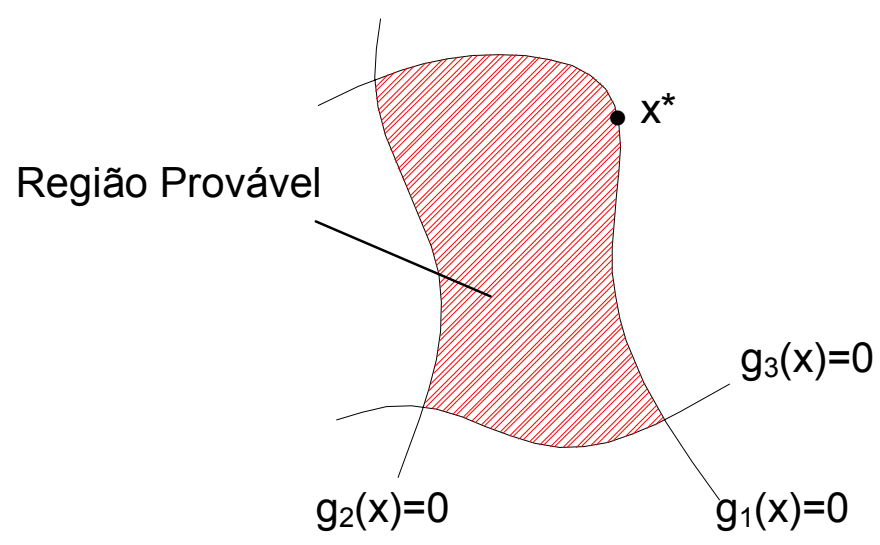

Figura 3.7 - Exemplo de restrições ativas e inativas 


\subsubsection{Método dos Multiplicadores de Lagrange}

$\mathrm{Na}$ essência, o método dos multiplicadores de Lagrange em cálculo de variações é uma extensão direta do método para minimização restrita em cálculo diferencial. Para uma função objetivo $f(\bar{x})$ de $n$ variáveis de projeto ser um mínimo, as variações na função objetivo deve desaparecer.

$$
\mathrm{df}=\frac{\partial \mathrm{f}}{\partial \mathrm{x}_{1}} \mathrm{dx_{1 }}+\frac{\partial \mathrm{f}}{\partial \mathrm{x}_{2}} \mathrm{dx_{2 }}+\ldots+\frac{\partial \mathrm{f}}{\partial \mathrm{x}_{\mathrm{n}}} \mathrm{dx} \mathrm{x}_{\mathrm{n}}=0
$$

Entretanto, os termos da derivada não podem ser um conjunto nulo porque as variações nas variáveis de projeto $\left(\mathrm{dx}_{1}, \mathrm{dx}_{2}, \ldots, \mathrm{dx_{n }}\right)$ são dependentes entre si através de equações de restrições.

Para simplificar, assume-se somente uma única relação de restrição $h(\bar{x})=0$, tem-se as seguintes variações nas variáveis de projeto:

$$
\mathrm{dh}=\frac{\partial \mathrm{h}}{\partial \mathrm{x}_{1}} \mathrm{dx} \mathrm{x}_{1}+\frac{\partial \mathrm{h}}{\partial \mathrm{x}_{2}} \mathrm{dx_{2 }}+\ldots+\frac{\partial \mathrm{h}}{\partial \mathrm{x}_{\mathrm{n}}} \mathrm{dx} \mathrm{x}_{\mathrm{n}}=0
$$

Quando se multiplica a Equação (3.6) por uma constante arbitrária $\lambda$, chamada de multiplicador de Lagrange, e somada à Equação (3.5), obtém-se:

$$
\left(\frac{\partial \mathrm{f}}{\partial \mathrm{x}_{1}}+\lambda \frac{\partial \mathrm{h}}{\partial \mathrm{x}_{1}}\right) \mathrm{dx_{1 }}+\left(\frac{\partial \mathrm{f}}{\partial \mathrm{x}_{2}}+\lambda \frac{\partial \mathrm{h}}{\partial \mathrm{x}_{2}}\right) \mathrm{dx_{2 }}+\ldots+\left(\frac{\partial \mathrm{f}}{\partial \mathrm{x}_{\mathrm{n}}}+\lambda \frac{\partial \mathrm{h}}{\partial \mathrm{x}_{\mathrm{n}}}\right) \mathrm{dx_{n }}=0
$$

Se $\lambda$ for determinado, as quantidades dentro de cada parêntese desapareçam para satisfazer à equação anterior. Isto resultará em $n$ equações para $(n+1)$ incógnitas, as $n$ variáveis de projeto e o multiplicador desconhecido $\lambda$. 
A relação de restrição $h(\bar{x})=0$ fornece $a(n+1)$ ésima relação. As Equações (3.7) e (3.4) são exatamente as que seriam obtidas por uma minimização sem restrições de uma função auxiliar $(f+\lambda h)$ com respeito às variáveis de projeto e multiplicador de Lagrange $\lambda$.

Para múltiplas funções de restrição, há um multiplicador de Lagrange $\lambda$ para cada função de restrição. Conseqüentemente, um problema de otimização de uma função objetivo com $n$ variáveis de projeto mais $n_{e}$ restrições de igualdade estabelecidas em (3.4) é equivalente a um problema sem restrições com uma função auxiliar:

$$
L(x, \lambda)=f(x)+\sum_{j=1}^{n_{e}} \lambda_{j} h_{j} .
$$

Os valores ótimos das variáveis de projeto podem ser obtidos pela resolução de um sistema de $\left(n+n_{e}\right)$ equações:

$$
\begin{array}{ll}
\frac{\partial L}{\partial x_{i}}=0, & i=1, \ldots, n \\
\frac{\partial L}{\partial \lambda_{j}}=0, & j=1, \ldots, n_{e}
\end{array}
$$

para $\left(n+n_{e}\right)$ incógnitas.

\subsubsection{Condições para Mínima Restrição}

Considera-se primeiro um problema de otimização com duas variáveis e uma única restrição de igualdade:

$$
\begin{array}{ll}
\min \mathrm{f}(\mathrm{x}), & \text { para } \mathrm{x}=\left(\mathrm{x}_{1}, \mathrm{x}_{2}\right) \in \mathrm{R}^{2}, \\
\text { sujeito à } & \mathrm{c}\left(\mathrm{x}_{1}, \mathrm{x}_{2}\right)=0 .
\end{array}
$$


A região provável para este problema consiste naqueles pontos de $R^{2}$ para os quais a equação $\mathrm{c}(\mathrm{x})=0$ é válida, supõe-se que se possa resolver esta equação para $x_{2}$ em termos de $x_{1}$ :

$$
x_{2}=y\left(x_{1}\right)
$$

Portanto, para qualquer valor de $\mathrm{x}_{1}$, pode-se calcular o único valor de $\mathrm{x}_{2}$ que faz $\left(x_{1}, x_{2}\right)$ provável, pode-se assim considerar $x_{1}$ como um valor independente (ou variável de decisão) e $x_{2}$ como uma variável dependente (ou variável de estado). Deste modo, tem-se:

$$
F\left(x_{1}\right)=f\left\{x_{1}, y\left(x_{1}\right)\right\}
$$

um problema restrito e equivalente ao problema sem restrições:

$$
\min F\left(x_{1}\right), \quad \text { para todo } x_{1} \in R^{1}
$$

Se $x_{1}{ }^{*}$ for uma solução para este problema, então $F^{\prime}\left(x_{1}{ }^{*}\right)=0$. Mas

$$
\frac{\mathrm{dF}}{\mathrm{dx}_{1}}=\frac{\partial \mathrm{f}}{\partial \mathrm{x}_{1}}+\frac{\partial \mathrm{f}}{\partial \mathrm{x}_{2}} \frac{\mathrm{dy}}{\mathrm{dx}_{1}}
$$

Assim, pode-se obter alguma informação em $y^{\prime}\left(x_{1}\right)$ pela observação de que

$$
\mathrm{C}\left(\mathrm{x}_{1}\right) \equiv \mathrm{C}\left(\mathrm{x}_{1}, \mathrm{y}\left(\mathrm{x}_{1}\right)\right)=0, \quad \text { para todo } \mathrm{x}_{1}
$$

e, conseqüentemente, que

$$
\frac{d C}{d x_{1}}=\frac{\partial c}{\partial x_{1}}+\frac{\partial c}{\partial x_{2}} \frac{d y}{d x_{1}}=0
$$




$$
\frac{\mathrm{dy}}{\mathrm{dx}_{1}}=-\frac{\partial \mathrm{c}}{\partial \mathrm{x}_{1}} / \frac{\partial \mathrm{c}}{\partial \mathrm{x}_{2}}
$$

A condição $F^{\prime}\left(x_{1}{ }^{*}\right)=0$ pode ser escrita como:

$$
\frac{d f}{d x_{1}}-\left(\frac{\partial f}{\partial x_{2}} / \frac{\partial c}{\partial x_{2}}\right) \frac{\partial c}{\partial x_{1}}=0
$$

e ao se adicionar a identidade:

$$
\frac{d f}{d x_{2}}-\left(\frac{\partial f}{\partial x_{2}} / \frac{\partial c}{\partial x_{2}}\right) \frac{\partial c}{\partial x_{2}}=0
$$

chega-se à relação:

$$
\nabla f-\lambda \nabla c=0
$$

onde $\lambda=\frac{\partial f}{\partial x_{2}} / \frac{\partial c}{\partial x_{2}}$ é o multiplicador de Lagrange correspondendo a equação restrita e o lado esquerdo é conhecido como a derivada restrita. Para o caso mais geral onde $x$ é um vetor de ordem $n$ e c um vetor de ordem $m$, a condição necessária para $\bar{x}^{*}$ ser uma solução é que lá deveria estar o vetor de ordem $\mathrm{m}, \lambda$ dos multiplicadores de Lagrange, tal que

$$
\nabla f\left(x^{*}\right)-\lambda^{\top} \nabla c\left(x^{*}\right)=0
$$

A seguir apresenta-se uma interpretação dos multiplicadores de Lagrange usada quando se consideram restrições de desigualdade. Retornando ao problema com duas variáveis e uma restrição, generaliza-se a restrição para:

$$
c(x)=b
$$


e observa-se que a solução $\bar{x}$ * deve ser uma função da quantidade $b$ que ocorre na restrição; então pode-se escrever:

$$
\bar{x}^{*}=X(b)
$$

Para todo $b, X(b)$ deve satisfazer:

$$
\begin{gathered}
c\{X(b)\}=b, \\
\nabla f\{X(b)\}-\lambda \nabla c\{X(b)\}=0, \\
\frac{d f\{X(b)\}}{d b}=\frac{\partial f}{\partial x_{1}} X_{1}^{\prime}(b)+\frac{\partial f}{\partial x_{2}} X^{\prime}(b)=\nabla f\{X(b)\}^{\top} \frac{d X}{d b}
\end{gathered}
$$

e

$$
1=\frac{\mathrm{dc}\{X(b)\}}{d b}=\nabla c\{X(b)\}^{\top} \frac{d X}{d b}
$$

Empregando estas duas últimas equações, nota-se que

$$
\nabla f\{X(b)\} \frac{d X}{d b}-\lambda \nabla c\{X(b)\} \frac{d X}{d b}=0
$$

e obtém-se:

$$
\lambda=\frac{\mathrm{df}\{\mathrm{x}(\mathrm{b})\}}{\mathrm{db}} .
$$

No caso mais geral quando há m restrições, tem-se:

$$
\lambda_{i}=\frac{d f\{x(b)\}}{d b_{i}}, \quad \text { para } i=1,2, \ldots, m
$$




\section{Teorema 6}

Se $\overline{\mathrm{x}}$ * for um ponto irregular do problema de otimização restrita geral (3.6) e for também um ponto de minimização, então existirá $\lambda \in R^{m}$ e $\mu \in R^{p}$ tal que

$$
\begin{gathered}
\nabla f\left(x^{*}\right)-\lambda^{T} \nabla g\left(x^{*}\right)-\bar{u}^{T} \nabla h\left(x^{*}\right)=0, \\
\lambda^{T} \nabla g\left(x^{*}\right)=0, \\
\lambda \geq 0, \quad g\left(x^{*}\right) \geq 0 \quad \text { e } \quad h\left(x^{*}\right)=0 .
\end{gathered}
$$

Estas são conhecidas como as condições de Kuhn-Tucker.

Se $f$ for convexo, os $g_{i}$ forem côncavos, e os $h_{i}$ forem lineares, tal problema será de programação convexa, e as condições de Kuhn-Tucker considerarem que $\overline{\mathrm{x}}$ * seja uma solução para o problema.

Se em alguma vizinhança $\mathcal{N}$ de $\bar{x}^{*}$, f for convexo, os $g_{i}$ serão côncavos, e os $h_{i}$ serão lineares, então as condições de Kuhn-Tucker serão suficientes para provar que $\bar{x}$ * será uma solução local para o problema (3.5). 


\section{ANÁLISE DA CONFIABILIDADE}

\subsection{Introdução}

A segurança de uma estrutura é função do máximo carregamento, ou combinação de cargas, que pode ser imposta durante toda a sua vida útil. $A$ segurança estrutural também depende da resistência de seus componentes. Como a máxima carga existente e a atual capacidade estrutural são difíceis de se prever exatamente, pois estão sujeitas à incertezas, a absoluta segurança de uma estrutura não é garantida.

Realisticamente, segurança e utilização podem estar asseguradas somente em termos da probabilidade de que a resistência existente, ou capacidade estrutural seja adequada para resistir à máxima carga existente. Problemas de confiabilidade envolvem uma determinação ou garantia da adequação de um sistema durante sua vida útil.

Portanto, pode-se definir confiabilidade como uma medida probabilística de garantia do desempenho, ou seja, a segurança do desempenho pode ser realisticamente estabelecida somente em termos de probabilidade.

Assim, a análise estrutural probabilística é, segundo DITLEVSEN et al. (1996), a arte de formular um modelo matemático dentro do qual pode-se propor e responder o seguinte problema: "qual é a probabilidade de que uma estrutura comporte-se de uma forma especificada, uma vez que uma ou mais das propriedades dos materiais que a constituem ou ainda das suas dimensões geométricas, são de natureza não completamente conhecidas ou aleatórias?". 


\subsection{Fundamentos Estatísticos da Confiabilidade}

\subsubsection{Espaço Amostral}

Espaço amostral $(\Omega)$ de um experimento realizado sob condições fixas, é o conjunto de todos os resultados possíveis do experimento, entendendo-se por resultado possível todo resultado elementar e indivisível do experimento.

\subsubsection{Evento}

Seja $\Omega$ o espaço amostral de um experimento. Todo subconjunto $A \subset \Omega$ será chamado de evento $(\omega)$. O conjunto $\Omega$ é o evento certo, o subconjunto $\varnothing$ é o evento impossível e se $\omega \in \Omega$ o evento é dito elementar e indivisível.

Seja A um evento do espaço amostral $\Omega$, então quando se atribui uma probabilidade ao evento $A$, este passa a ser chamado de evento aleatório.

\subsection{3 $\sigma$-Álgebra}

$\sigma$-Álgebra $(\mathrm{A})$ de subconjuntos do conjunto, não-vazio, $\Omega$ é a classe de subconjuntos de $\Omega$ satisfazendo as propriedades:

- $\Omega \in A$;

- $\quad$ se $A \in A \Rightarrow A^{c} \in A$;

- $\quad$ se $A_{1}, A_{2}, A_{3}, \ldots . . A \Rightarrow \bigcup_{i=1}^{\infty} A_{i} \in A$. 


\subsubsection{Variável Aleatória}

Uma variável $X$ em um espaço de probabilidade $(\Omega, \mathrm{A}, \mathrm{P})$ é uma função real definida no espaço $\Omega$, tal que o evento $[\mathrm{X} \leq \mathrm{x}$ ] é um evento aleatório, $\forall \mathrm{X}$ $\in R$, isto é, a função $X: \Omega \rightarrow R$ é uma variável aleatória (v.a) se o evento [ $X \leq$ $x] \in A, \forall x \in R$.

A v.a X é chamada de discreta quando o seu contradomínio é um conjunto finito ou infinito enumerável, ou melhor, se existe um conjunto finito ou infinito enumerável $\left\{x_{1}, x_{2}, x_{3}, \ldots\right\} \subset R$, tal que $X(\omega) \in\left\{x_{1}, x_{2}, x_{3}, \ldots\right\}, \forall \omega \in \Omega$.

A v.a X é chamada de contínua quando o seu contradomínio é um conjunto infinito.

\subsubsection{Probabilidade}

Seja $\Omega$ um conjunto finito e A um subconjunto do espaço amostral $\Omega$; A $\in \mathrm{P}(\Omega)$, então se todos os resultados elementares de $\Omega$ são equiprováveis, a medida da probabilidade de ocorrência do evento A é dada por:

$$
P(A)=\frac{\# A}{\# \Omega}, A \in A \text {. }
$$

Espaço de probabilidade é o trio $(\Omega, \mathrm{A}, \mathrm{P})$, onde $\Omega, \mathrm{A}$ e $\mathrm{P}$ foram definidos anteriormente.

\subsubsection{Propriedades da Probabilidade}

Tem-se as seguintes propriedades da probabilidade: 
- $P(A) \geq 0$;

- $P(\Omega)=1$;

- $\quad$ se $A$ e $B \in A$ e são disjuntos $\Rightarrow P(A \cup B)=P(A)+P(B)$;

- $\quad$ se $A_{1}, A_{2}, A_{3}, \ldots, A_{n} \in A$ e são disjuntos $\Rightarrow P\left(\bigcup_{i=1}^{n} A_{i}\right)=\sum_{i=1}^{n} P\left(A_{i}\right)$

- $\quad$ se $A_{1}, A_{2}, A_{3}, \ldots \in A$ e são disjuntos $\Rightarrow P\left(\bigcup_{i=1}^{\infty} A_{i}\right)=\sum_{i=1}^{\infty} P\left(A_{1}\right)$.

- $\quad$ se $A$ é um evento aleatório, então a probabilidade de $A$ não ocorrer é dada por $P\left(A^{C}\right)=1-P(A)$;

- $\quad$ se $A$ é um evento aleatório, então $0 \leq P(A) \leq 1$;

- $\quad$ se $A_{1} \subset A_{2} \Rightarrow P\left(A_{1}\right) \leq P\left(A_{2}\right)$ e $P\left(A_{2}-A_{1}\right)=P\left(A_{2}\right)-P\left(A_{1}\right)$;

- $P\left(A_{1} \cup A_{2}\right)=P\left(A_{1}\right)+P\left(A_{2}\right)-P\left(A_{1} \cap A_{2}\right)$;

- $\quad P\left(\bigcup_{i=1}^{n} A_{i}\right) \leq \sum_{i=1}^{n} P\left(A_{i}\right)$;

- $\quad P\left(\bigcup_{i=1}^{\infty} A_{i}\right) \leq \sum_{i=1}^{\infty} P\left(A_{i}\right)$

- $P\left(A_{1} \cup A_{2} \cup \ldots \cup A_{n}\right)=P\left({ }_{i=1}^{n} A_{i}\right)=\sum_{r=1}^{n}(-1)^{r-1} S_{r}=S_{1}-S_{2}+S_{3}-\ldots+(-1)^{r-1} S_{n}$;

- $\quad P\left(\bigcap_{i=1}^{n} A_{1}\right) \geq 1-\sum_{i=1}^{n} P\left(A_{i}^{c}\right)$

- $\quad P\left(\bigcap_{i=1}^{\infty} A_{i}\right) \geq 1-\sum_{i=1}^{\infty} P\left(A_{i}^{c}\right)$

- $\quad$ continuidade em probabilidade: "Seja a seqüência $\left\{A_{i}\right\}, i=1,2,3$, $\ldots$, onde $A_{i} \in A \forall i$, então se $A_{i}$ convergir para $A$, isto implicará que $P\left(A_{i}\right)$ converge para $P(A)$; se $A_{i}$ divergir de $A$, então $P\left(A_{i}\right)$ divergirá de $P(A)$.

\subsubsection{Probabilidade Condicional e Independência de Eventos}

Sejam o espaço de probabilidade $(\Omega, A, P)$ e os eventos $A, B \in A$ com $P(B)>0$. Denomina-se probabilidade condicional do evento $A$ dado o evento $B$ 
a razão entre a probabilidade da ocorrência simultânea dos eventos e a probabilidade do evento $B$, ou seja:

$$
P(A \mid B)=\frac{P(A \cap B)}{P(B)}, \quad A, B \in A \text {. }
$$

Se $P(B)=0, P(A \mid B)$ pode ser arbitrariamente definida. A maioria dos livros faz $P(A \mid B)=0$, mas é mais conveniente, pela independência, fazer-se $P(A \mid B)=P(A)$.

Como $\mathrm{P}(\mathrm{A} \mid \mathrm{B})$ é uma probabilidade, são válidas todas as propriedades de probabilidade.

Como $P(A \mid B)=\frac{P(A \cap B)}{P(B)}$, então a probabilidade da ocorrência simultânea dos eventos A e B é dada por:

$$
P(A \cap B)=P(A) \cdot P(B \mid A)=P(B) \cdot P(A \mid B)
$$

\subsubsection{Independência de Eventos}

Seja o espaço de probabilidade $(\Omega, A, P)$, então os eventos aleatórios $A$ e $B$ são estocasticamente independentes se $P(A \cap B)=P(A) \cdot P(B)$, ou seja, $P(B \mid A)=P(B)$ e $P(A \mid B)=P(A)$. 


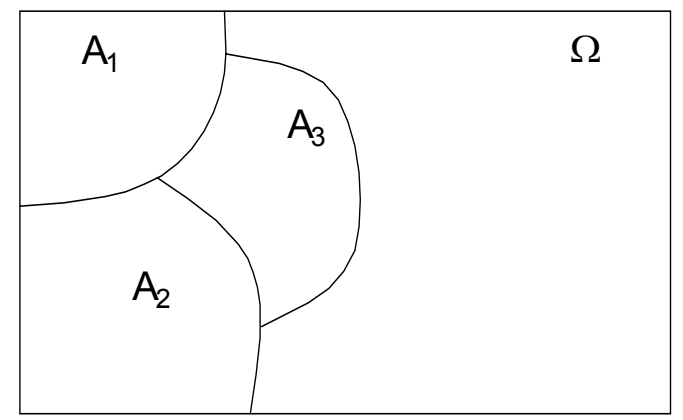

Figura 4.1 - Partição do espaço amostral $\Omega$

Sejam $A_{1}, A_{2}, A_{3}, \ldots$ eventos aleatórios mutuamente exclusivos e exaustivos, isto é, os $A_{i}$ são disjuntos e $\bigcup_{i=1}^{\infty} A_{i}=\Omega$, então os eventos $A_{i}$ formam uma partição do espaço amostral $\Omega$, como mostrado na Figura 4.1.

Através da Figura 4.2, admitindo-se que a seqüência $A_{1}, A_{2}, A_{3}, \ldots$ seja finita ou infinita enumerável, pode se observar que:

- $A_{i}$ e $A_{i}^{c}$ formam uma partição, $\forall A_{i} \in A$;

- $\quad \forall$ evento $B \in A$ tem-se $B=\bigcup_{i}\left(A_{i} \cap B\right)$, pois os $A_{i}$ são disjuntos, então os $B \cap A_{i}$ também são disjuntos e $B=\bigcup_{i} B \cap A_{i}$, logo:

$$
P(B)=\sum_{i} P\left(A_{i} \cap B\right)=\sum_{i} P\left(A_{i}\right) \cdot P\left(B \mid A_{i}\right)
$$

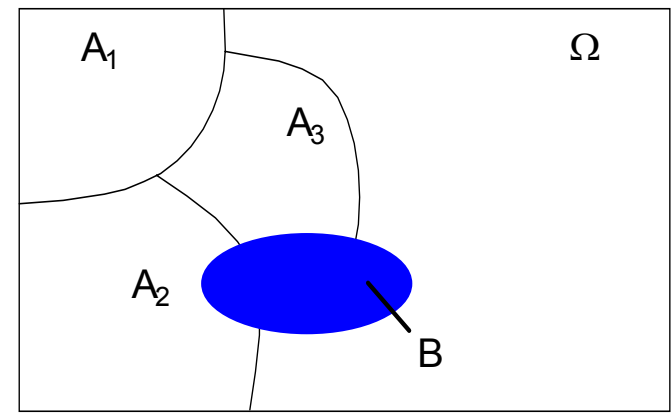

Figura 4.2 - Partição do espaço amostral $\Omega$ 


\subsubsection{Teorema da Probabilidade Total}

Se a seqüência (finita ou infinita enumerável) de eventos aleatórios $A_{1}$, $A_{2}, A_{3}, \ldots$ forma uma partição do espaço amostral $\Omega$, então a probabilidade de um evento B contido em $\Omega$ é dada por:

$$
P(B)=\sum_{i} P\left(A_{i}\right) \cdot P\left(B \mid A_{i}\right)
$$

O Teorema da Probabilidade Total é utilizado quando se conhecem todas as $P\left(A_{i}\right)$ e as $P\left(B \mid A_{i}\right)$, mas se desconhece diretamente $P(B)$ e com base nesse resultado é possível calcular a probabilidade do evento $A_{j}$ dada $a$ ocorrência do evento $B$, pela fórmula:

$$
P\left(A_{j} \mid B\right)=\frac{P\left(A_{j} \cap B\right)}{P(B)}=\frac{P\left(A_{j}\right) \cdot P\left(B \mid A_{j}\right)}{P(B)}=\frac{P\left(A_{j}\right) \cdot P\left(B \mid A_{j}\right)}{\sum_{i} P\left(A_{i}\right) \cdot P\left(B \mid A_{i}\right)} .
$$

que é conhecida como fórmula de Bayes ou Teorema de Bayes.

\subsubsection{Função Distribuição}

A função distribuição ou função distribuição acumulada, da v.a $X$ é definida por $F_{X}(x)=P_{X}(X \leq x)$. A Figura 4.3 ilustra esta definição para uma v.a contínua. 


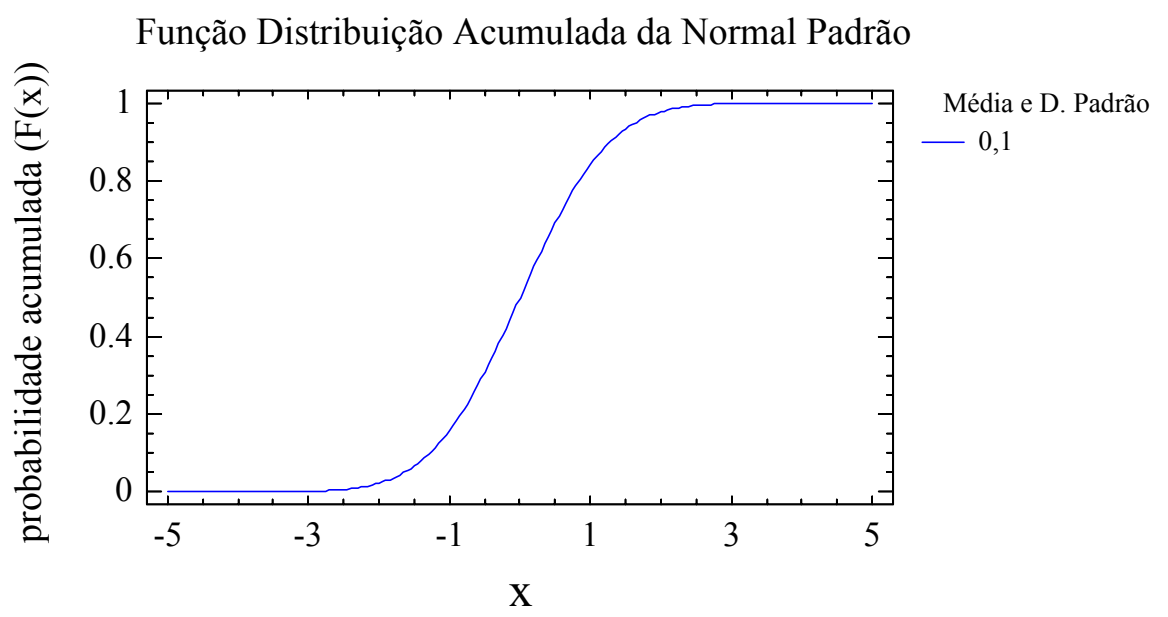

Figura 4.3 - Função distribuição acumulada

A função distribuição $F_{X}(x)$ tem as seguintes propriedades:

- $\quad$ a função distribuição da v.a $X, F_{X}(x)=P_{X}(X \leq X)$, é não decrescente, isto é, se $x \leq y$, então $F_{X}(x) \leq F_{X}(y)$;

- $\quad$ a função distribuição da v.a $X, F_{X}(X)=P_{X}(X \leq X)$, é contínua à direita, isto é, se $x_{n}$ divergir de $x$, então $F_{X}\left(x_{n}\right)$ divergirá de $F_{X}(X)$;

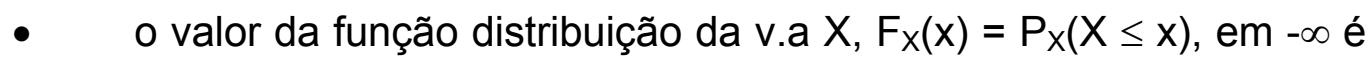
$F_{x}(-\infty)=0$ e em $\infty$ é $F_{X}(\infty)=1$, ou seja, se $x_{n}$ divergir de $x$, então $F_{X}\left(x_{n}\right)$ divergirá de 0 e se $x_{n}$ divergir de $x$, então $F_{X}\left(x_{n}\right)$ divergirá de 1.

\subsubsection{Função de Probabilidade e Função Densidade de Probabilidade}

Uma vez que uma v.a assume um valor de seu contradomínio com uma certa probabilidade, tem-se que as probabilidades são associadas a valores da variável aleatória discreta por uma função de probabilidade (f.p.). As probabilidades são associadas a intervalos de valores de uma variável aleatória contínua por uma função densidade de probabilidade (f.d.p.). 
A função de probabilidade da v.a discreta $X$, representada por $P(X=x)=$ $\mathrm{p}(\mathrm{x})$, é uma função, tal que para $\mathrm{X}(\omega) \in\left\{\mathrm{x}_{1}, \mathrm{x}_{2}, \mathrm{x}_{3}, \ldots\right\}, \forall \omega \in \mathrm{A}$, tem-se $\mathrm{p}\left(\mathrm{x}_{\mathrm{i}}\right) \geq 0$ e $\sum_{i=1}^{\infty} p\left(x_{i}\right)=1$

A função densidade de probabilidade da v.a contínua $X$, representada por $f_{X}(x)$, é uma função, tal que $f_{X}(x) \geq 0$ e $\int_{-\infty}^{\infty} f(x) d x=1$. E como a função distribuição acumulada da v.a. $X$ é $P(X \leq x)=F_{X}(x)=\int_{-\infty}^{x} f(t) d t$, tem-se que $f(x)=$ $\frac{\partial \mathrm{F}_{\mathrm{X}}(\mathrm{x})}{\partial \mathrm{x}}$. A Figura 4.4 ilustra a definição dada.

Função Dens. de Probabilidade da Normal Padrão

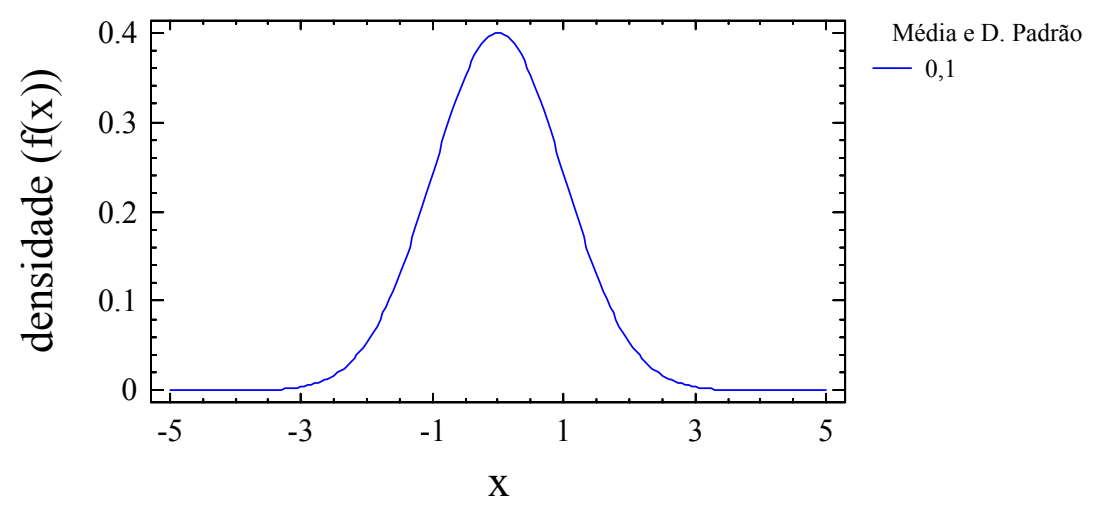

Figura 4.4 - Função densidade de probabilidade da Normal Padrão

\subsubsection{Funções Conjuntas}

Sejam $X_{1}, X_{2}, \ldots, X_{k}$ v.a's, todas definidas no mesmo espaço de probabilidade $(\Omega, \mathrm{A}, \mathrm{P})$, então a função distribuição acumulada conjunta é definida por $F\left(x_{1}, x_{2}, \ldots, x_{k}\right)=P\left(X_{1} \leq x_{1}, X_{2} \leq x_{2}, \ldots, X_{k} \leq x_{k}\right)$.

Sejam $X_{1}, X_{2}, \ldots, X_{k}$ v.a's discretas, todas definidas no mesmo espaço de probabilidade $(\Omega, A, P)$, então a função de probabilidade conjunta é definida por $P\left(x_{1}, x_{2}, \ldots, x_{k}\right)=P\left(X_{1}=x_{1}, X_{2}=x_{2}, \ldots ., X_{k}=x_{k}\right)$. 
Sejam $X_{1}, X_{2}, \ldots, X_{k}$ v.a's contínuas, todas definidas no mesmo espaço de probabilidade $(\Omega, \mathrm{A}, \mathrm{P})$, tais que $\mathrm{F}\left(\mathrm{x}_{1}, \mathrm{x}_{2}, \ldots, \mathrm{x}_{\mathrm{k}}\right)=\mathrm{P}\left(\mathrm{X}_{1} \leq \mathrm{x}_{1}, \mathrm{X}_{2} \leq \mathrm{x}_{2}, \ldots\right.$. $\left.X_{k} \leq x_{k}\right)=\int_{-\infty}^{x_{k}} \ldots \ldots . \int_{-\infty}^{x_{1}} f\left(x_{1}, x_{2}, \ldots \ldots, x_{k}\right) d x_{1} \ldots . d x_{k}$, então $f\left(x_{1}, x_{2}, \ldots ., x_{k}\right)$ é denominada função densidade de probabilidade conjunta das v.a's $X_{1}, X_{2}, \ldots$, $X_{\mathrm{k}}$.

\subsubsection{Distribuição de Probabilidade Normal (Gaussiana)}

Uma v.a X tem distribuição Normal ou Gaussiana quando a sua f.d.p. tem a forma:

$$
f_{X}(x)=\frac{1}{\sigma \sqrt{2 \pi}} e^{-\frac{(x-\mu) 2}{2 \sigma^{2}}}, x \in R, \mu \in R \text { e } \sigma \in R^{+}
$$

Na distribuição Normal, a probabilidade da v.a X assumir um valor entre $a$ e b $(a<b)$ é dado por:

$$
P(a<X<b)=\int_{a}^{b} f(x) d x
$$

Como é difícil trabalhar com todos os membros da família Normal, prefere-se trabalhar com a Normal Reduzida ou Normal Padrão. Esta v.a é representada por $Z$ e tem a seguinte f.d.p.:

$$
f_{z}(z)=\frac{1}{\sqrt{2 \pi}} e^{-\frac{z^{2}}{2}}, \quad z \in R
$$

A distribuição de $Z$ tem média e variância iguais a $\mu=0$ e $\sigma^{2}=1$, respectivamente, e essa v.a é obtida da transformação $Z=(X-\mu) / \sigma$, onde $X$ $\mathrm{N}\left(\mu, \sigma^{2}\right)$. 


\subsubsection{Distribuição de Soma e Diferença de Duas Variáveis Aleatórias (Convolução)}

A distribuição de uma variável aleatória que seja igual à soma ou à diferença entre duas outras variáveis aleatórias é muito útil em aplicações e tem forma definida pelo resultado a seguir.

\section{Resultado 1}

Sejam $X$ e $Y$ v.a's com f.d.p. conjunta $f(x, y)$ e sejam as v.a's $Z=X+Y$ e $V=X-Y$, então a f.d.p. de $Z$ é $f_{Z}(Z)=\int_{-\infty}^{\infty} f_{X, Y}(X, Z-X) d x=\int_{-\infty}^{\infty} f_{X, Y}(Z-y, y) d y$ e $a$ f.d.p. da v.a $V$ é dada por $f_{V}(v)=\int_{-\infty}^{\infty} f_{X, Y}(x, z-x) d x=\int_{-\infty}^{\infty} f_{X, Y}(v+y, y) d y$.

\section{PROVA}

Seja a f.d. da v.a $Z, F_{Z}(z)=P[Z \leq z]=P[X+Y \leq z]=\int_{-\infty}^{\infty}\left[\int_{-\infty}^{Z-x} f_{X, Y}(x, y) d y\right] d x=$ $\int_{-\infty}^{\infty}\left[\int_{-\infty}^{z} f_{x, Y}(x, u-x) d u\right] d x$, com $y=u-x$. $E$ a f.d.p. de $Z$ é igual $f_{Z}(z)=\frac{\partial F_{Z}(z)}{\partial z}=$ $\frac{\partial}{\partial Z}\left\{\int_{-\infty}^{z}\left[\int_{-\infty}^{\infty} f_{X, Y}(x, u-X) d x\right] d u\right\}=\int_{-\infty}^{\infty} f_{X, Y}(x, \quad Z-x) d x$ e se as v.a's $X$ e $Y$ são independentes, tem-se que $f_{Z}(z)=\int_{-\infty}^{\infty} f_{X, Y}(x, z-x) d x=\int_{-\infty}^{\infty} f_{X}(z-y) f_{Y}(y) d y=\int_{-\infty}^{\infty} f_{Y}(z-$ $x) f_{x}(x) d x$ e nesse caso de independência o resultado é chamado de convolução, ou seja, a função $f_{Z}($.$) é chamada de convolução das funções f_{X}($. e $f_{Y}($.$) .$ 


\subsubsection{Esperança e Variância de uma Variável Aleatória}

Seja uma variável aleatória $X$ discreta que assume valores no conjunto $\left\{\mathrm{x}_{1}, \mathrm{x}_{2}, \mathrm{x}_{3}, \ldots,\right\}$. Chama-se valor médio ou esperança matemática de $\mathrm{X}$ ao valor:

$$
\mu=E(X)=\sum_{i=1}^{\infty} x_{i} \cdot P_{X}\left(x_{i}\right)=\sum_{i=1}^{\infty} x_{i} \cdot P_{X}\left(X=x_{i}\right)
$$

Chama-se variância da v.a X ao valor :

$$
\sigma^{2}=V(X)=E[X-E(X)]^{2}=\sum_{i=1}^{n}\left[x_{i}-E(X)\right]^{2} \cdot P_{X}\left(X=x_{i}\right)=\sum_{i=1}^{n}\left[x_{i}-\mu\right]^{2} \cdot P_{X}\left(X=x_{i}\right) .
$$

O desvio padrão da v.a X é a raiz quadrada da variância, ou seja,

$$
\sigma=\sqrt{\mathrm{V}(\mathrm{X})}
$$

Uma relação importante é $V(X)=E\left(X^{2}\right)-[E(X)]^{2}$, onde

$$
E\left(X^{2}\right)=\sum_{i=1}^{n} x_{i}^{2} \cdot P\left(X=x_{i}\right)
$$

Da mesma forma, se a v.a for contínua, a esperança de X será dada por $E(X)=\mu=\int_{-\infty}^{\infty} x f(x) d x$ e a variância por $E(x-\mu)^{2}=\sigma^{2}=\int_{-\infty}^{\infty}(x-\mu)^{2} f(x) d x$.

É importante observar que a variância mede a dispersão (espalhamento) dos dados em torno da média $\mu=E(X)$ e o desvio padrão faz isto também, mas na mesma unidade de medida dos dados.

Se as v.a's $\mathrm{X}$ e $\mathrm{Y}$ não forem independentes, existirá uma diferença entre $E(X . Y)$ e $E(X) . E(Y)$, esta diferença será chamada de covariância e definida por 
$\operatorname{cov}(X, Y)=E[(X-E(X)) \cdot(Y-E(Y))] . E$ se $\operatorname{cov}(X, Y)=0$, as v.a's serão chamadas de não-correlacionadas.

A covariância entre as v.a's $X$ e $Y$ padronizadas é chamada de coeficiente de correlação $\rho=E\left[\left(\frac{X-E(X)}{\sigma_{x}}\right)\left(\frac{Y-E(Y)}{\sigma_{y}}\right)\right]$. Tem-se que $-1 \leq \rho \leq 1$ e ainda $\rho(X, Y)=1$, se e somente se $P(Y=a X+b)=1, \forall a>0$ e $\forall b \in R$. E $\rho(X, Y)=-1$, se e somente se $P(Y=a X+b)=1, \forall a<0$ e $\forall b \in R$.

O coeficiente de variação de uma variável aleatória é definido como o quociente entre o desvio padrão e a média (esperança matemática) da variável aleatória, ou seja,

$$
C V=\frac{\sigma}{\mu}
$$

\subsection{Análise da Confiabilidade em Projetos}

\subsubsection{Confiabilidade em Sistemas Estruturais}

Os problemas de confiabilidade estrutural podem ser formulados como a determinação da capacidade de um sistema satisfazer certas exigências. $\mathrm{Na}$ consideração da segurança de uma estrutura, o interesse é garantir que a resistência estrutural seja suficiente para resistir a máxima carga aplicada.

Tradicionalmente, a confiabilidade estrutural é alcançada através do uso de fatores ou margens de segurança e adotando suposições conservativas na etapa de projeto, isto é, pela determinação da mínima condição resistente que permanecerá adequada sob a máxima carga aplicada. As condições de mínima resistência e o máximo carregamento são definidas a partir de julgamentos subjetivos. Também, a adequação ou não das "margens" aplicadas pode ser 
avaliada ou calibrada somente em termos de experiências passadas com sistemas similares.

A aproximação tradicional é de difícil quantificação e necessita bases lógicas para o tratamento das incertezas. Além disso, para novos sistemas em que não há, a priori, bases para calibração, o problema para assegurar o desempenho, obviamente, torna-se difícil.

Na realidade, a determinação da resistência disponível, bem como as determinações da máxima carga aplicadas não são problemas simples. Estimativas e suposições são necessárias para estas propostas; nestes processos as incertezas são inevitáveis pela simples razão de que as informações são incompletas. A partir de tais incertezas, a resistência disponível e o carregamento não podem ser determinados precisamente; podem ser descritos como parte de respectivas faixas, ou populações, de possível resistência e carregamento.

A fim de representar o significado de incerteza, a resistência disponível e a carga existente podem ser modeladas como variáveis aleatórias. Nestes termos, a confiabilidade de um sistema pode ser mais realisticamente medida em termos de probabilidade. Por esse propósito, definem-se as seguintes variáveis aleatórias:

$$
\begin{aligned}
& X=\text { resistência, } \\
& Y=\text { solicitação. }
\end{aligned}
$$

Sendo assim, o objetivo da análise de confiabilidade é assegurar o evento $(X>Y)$ por todo a vida útil de uma estrutura. A garantia é possível somente em termos de probabilidade $\mathrm{P}(\mathrm{X}>\mathrm{Y})$. Esta probabilidade, conseqüentemente, representa uma medida realística da confiabilidade do sistema. 
Assume-se que as distribuições de probabilidade de $X$ e $Y ; F_{x}(x)$ ou $f_{x}(x)$ e $F_{y}(y)$ ou $f_{y}(y)$, respectivamente, são conhecidas. Os resultados necessários podem ser formulados a seguir.

\section{Resultado 2}

Seja a v.a X representando a capacidade de resistência de um determinado sistema estrutural sujeito à solicitação de carga $Y=y$, que também é uma v.a. Então se tem como a probabilidade de falha do sistema, se os eventos forem dependentes:

- $\quad$ no caso discreto:

$$
\mathrm{p}_{\mathrm{F}}=\mathrm{P}(\mathrm{X}<\mathrm{Y})=\sum_{\mathrm{y}} \mathrm{P}(\mathrm{X}<\mathrm{Y} \mid \mathrm{Y}=\mathrm{y}) \cdot \mathrm{P}(\mathrm{Y}=\mathrm{y})
$$

e na suposição mais realística de independência dos eventos, tem-se:

$$
p_{F}=P(X<Y)=\sum_{y} P(X<Y) \cdot P(Y=y)
$$

- $\quad$ no caso contínuo:

$$
p_{F}=P(X<y)=\int_{-\infty}^{\infty} F_{X}(y) \cdot f_{Y}(y) d y .
$$

E quando as v.a's contínuas são independentes, tem-se a probabilidade de falha igual a:

$$
p_{F}=P(X<y)=\int_{0}^{\infty} F_{X}(y) \cdot f_{Y}(y) d y .
$$




\section{PROVA}

Da definição de probabilidade condicional $P(A \mid B)=\frac{P(A \cap B)}{P(B)}, A, B \in A$, pode-se escrever:

$$
P(X<Y \mid Y=y)=\frac{P[(X<Y) \cap(Y=y)]}{P(Y=y)}
$$

e tem-se a probabilidade da ocorrência simultânea dos eventos $(X<Y)$ e $(Y=$ $\mathrm{y})$ :

$$
P[(X<Y) \cap(Y=y)]=P(Y=y) \cdot P(X<Y \mid Y=y)
$$

e considerando $\forall$ y, tem-se:

$$
p_{F}=P(X<Y)=\sum_{y} P(X<Y \mid Y=y) \cdot P(Y=y)
$$

como a resistência e a solicitação, $\mathrm{X}$ e $\mathrm{Y}$, são, em geral, estatisticamente independentes, isto é, $P(X<Y \mid Y=y)=P(X<y)$, obtém-se:

$$
p_{F}=P(X<Y)=\sum_{y} P(X<Y) \cdot P(Y=y)
$$

e no caso das v.a's serem contínuas, resulta da função distribuição de X:

$$
F_{X}(a)=P(X<a)=\int_{-\infty}^{a} f(x) d x
$$

e, conseqüentemente,

$$
F_{X}(y)=P(X<y)=\int_{-\infty}^{y} f_{x}(x) d x
$$


sendo que $\forall$ y resulta:

$$
p_{F}=P(X<y)=\int_{0}^{\infty} F_{X}(y) \cdot f_{Y}(y) d y
$$

O Resultado 5 também pode ser expresso em função da densidade de $X$ e da distribuição de $\mathrm{Y}$, já que se tem uma convolução e assim,

$$
p_{F}=P(X<y)=\int_{0}^{\infty}\left[1-F_{Y}(x)\right] . f_{X}(x) d x .
$$

A confiabilidade do sistema ou probabilidade de não falhar é medida por:

$$
p_{s}=1-p_{F}
$$

A Figura 4.5 retrata os argumentos abordados.

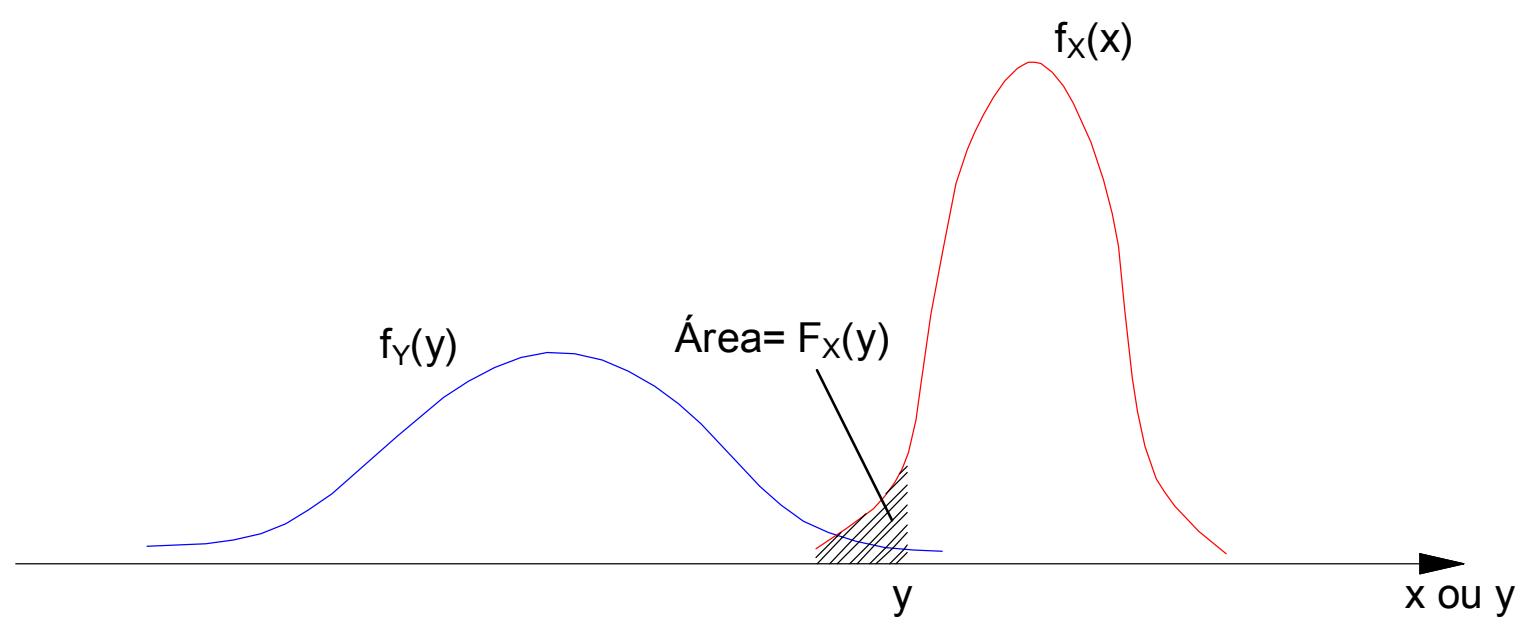

Figura 4.5 - f.d.p.'s $f_{X}(x)$ e $f_{Y}(y)$

É claro através da expressão $p_{s}=1-p_{F}$, que à medida que se aumenta a confiabilidade, diminui a probabilidade de falha. Observa-se pelas Figuras 4.4, 4.6 e 4.7 que a região coberta pela intersecção das curvas correspondentes às f.d.p's de $X$ e $Y$ depende das posições relativas de $f_{X}(x)$ e 
$f_{Y}(y)$ em R; ou melhor, à medida que os parâmetros de locação das densidades se afastam, $p_{F}$ diminui; e à medida que se aproximam, $p_{F}$ aumenta.

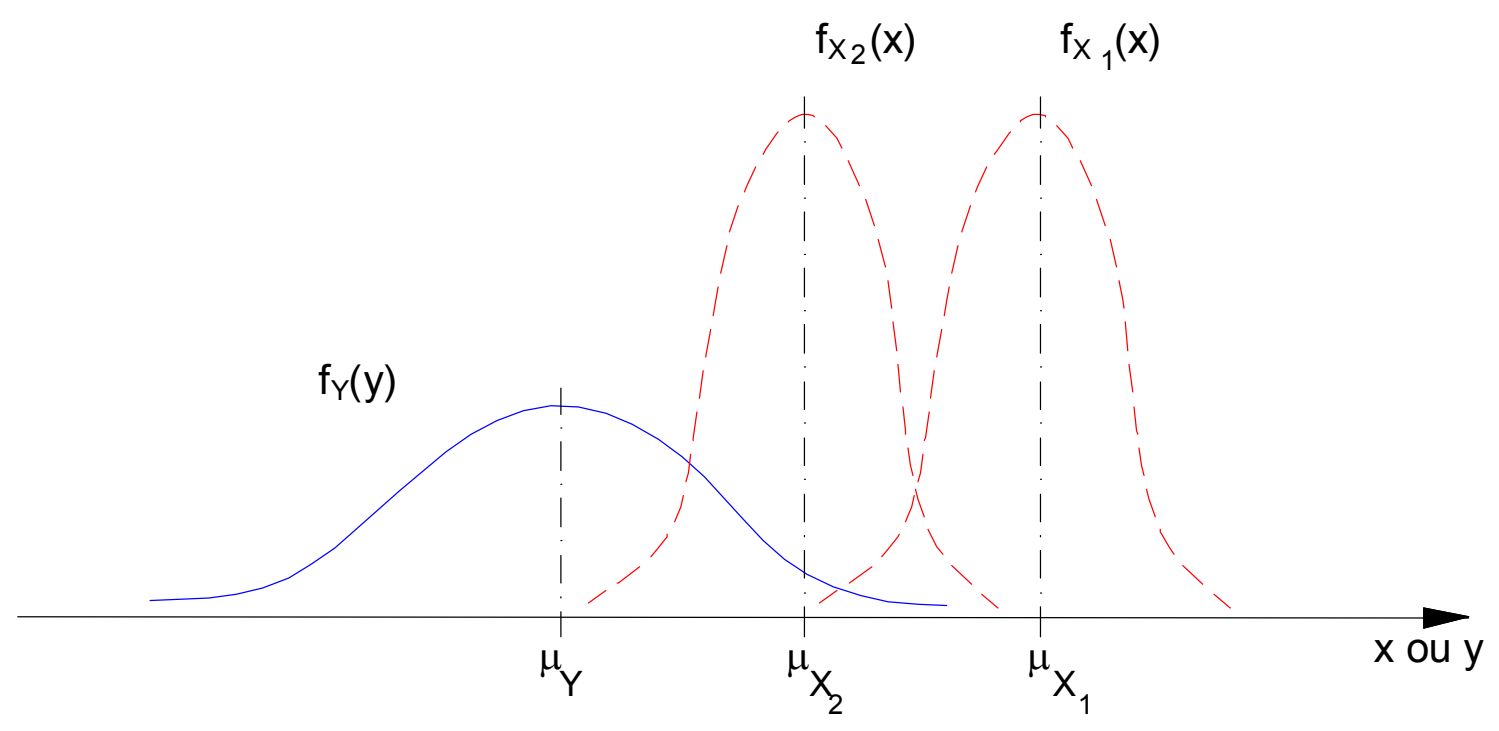

Figura 4.6 - Efeito da posição relativa entre $f_{X}(x)$ e $f_{Y}(y)$ em $p_{F}$

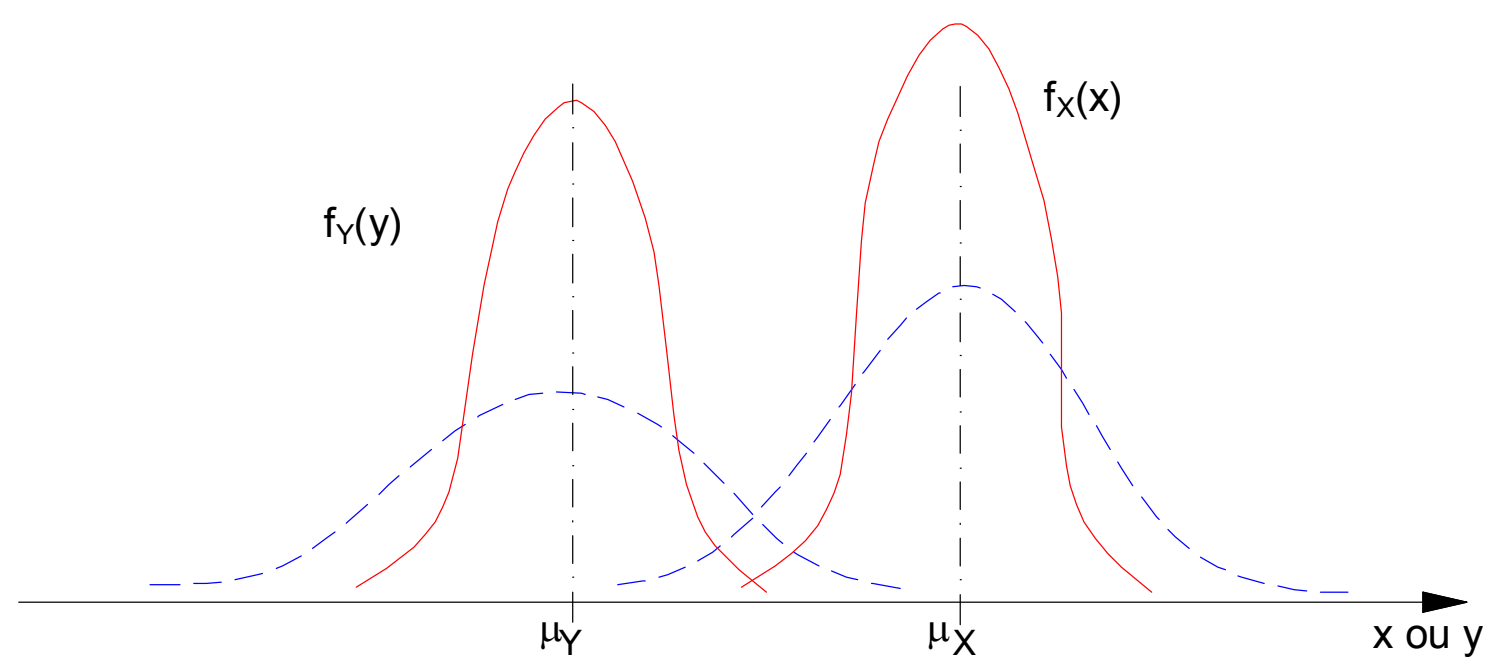

Figura 4.7 - Efeito das dispersões em $f_{X}(x)$ e $f_{Y}(y)$ em $p_{F}$

Conseqüentemente, um índice de confiabilidade para o sistema é função dos parâmetros de locação (médias) das distribuições. De modo que a posição relativa entre $f_{x}(x)$ e $f_{y}(y)$ pode ser medida pela razão $\mu_{x} / \mu_{y}$. Este quociente costuma ser chamado de "fator de segurança central". E, ainda, essa posição 
relativa pode ser medida pela diferença $\left(\mu_{\mathrm{x}}-\mu_{\mathrm{y}}\right)$, que é denominada "margem de segurança”.

É claro que a região coberta pela interseção das curvas depende, também, da dispersão das v.a's X e Y. Como é bem conhecido, as dispersões são medidas pelos desvios padrões das v.a's ou pelos seus quadrados, as variâncias. Um modo de relacionar os valores médios e as variâncias é usar os coeficientes de variação, tendo então medidas relativas de dispersão envolvendo os parâmetros dos quais depende a probabilidade de falha, $\mathrm{p}_{\mathrm{F}}$. Assim, têm-se as v.a's distribuídas conforme abaixo,

$$
X \sim\left(\mu_{X}, \sigma_{X}^{2}\right) \text { e } Y \sim\left(\mu_{Y}, \sigma_{Y}^{2}\right)
$$

e obtém-se os coeficientes de variação $C V_{X}=\frac{\sigma_{X}}{\mu_{X}}$ e $C V_{Y}=\frac{\sigma_{Y}}{\mu_{Y}}$.

A probabilidade de falha do sistema, $\mathrm{p}_{\mathrm{F}}$, também depende da forma de $f_{x}(x)$ e $f_{y}(y)$, entretanto na prática as informações sobre as formas dessas funções são muitas vezes mínimas e quando muito, dispõe-se de informações suficientes apenas para avaliar os principais parâmetros, ou seja, $\mu_{x}, \mu_{y}, \sigma_{x}^{2}$, $\sigma_{Y}^{2}$ e os correspondentes coeficientes de variação $C V_{x}$ e $C V_{y}$.

$\mathrm{Na}$ determinação de $\mathrm{p}_{\mathrm{F}}$, feita anteriormente, assume-se que as v.a's $\mathrm{X}$ e Y são independentes, porém essas variáveis podem ser correlacionadas e a determinação de $\mathrm{p}_{\mathrm{F}}$ ser diferente. Quanto se tem variáveis aleatórias correlacionadas, a probabilidade condicional de uma delas em relação à outra é diferente da probabilidade simples, ou seja:

$$
P(Y<X \mid X=x) \neq P(Y<x) \quad \text { e } \quad P(X<Y \mid Y=y) \neq P(X<y)
$$

Neste caso, a probabilidade de falha, $\mathrm{p}_{\mathrm{F}}$, pode ser determinada através de cálculo envolvendo a f.d.p. conjunta das v.a's X e Y, ou seja: 


$$
p_{F}=\int_{0}^{\infty}\left[\int_{0}^{y} f_{x, y}(x, y) d x\right] d y
$$

e tem-se a confiabilidade correspondente:

$$
p_{S}=1-p_{F}=\int_{0}^{\infty}\left[\int_{0}^{x} f_{x, y}(x, y) d y\right] d x
$$

A margem de segurança, $\mu_{\mathrm{x}}-\mu_{\mathrm{y}}$, pode ser usada na questão da "resistência-solicitação". A princípio, deve-se considerar a diferença entre as v.a's $M=X-Y$ que é também uma variável aleatória, pois é função de duas variáveis aleatórias. Dentro desta abordagem, entende-se por falha a ocorrência do evento aleatório $\mathrm{M}<0$ e, conseqüentemente, a probabilidade de falha é dada por:

$$
p_{F}=\int_{-\infty}^{0} f_{M}(m) d m=F_{M}(0)=p(M<0)
$$

onde $f_{M}(m)$ é a função densidade de probabilidade da variável aleatória $M$ e $F_{M}$ é a função distribuição de probabilidade. A probabilidade de falha, $\mathrm{p}_{\mathrm{F}}$, corresponde à área hachurada na Figura 4.8. 


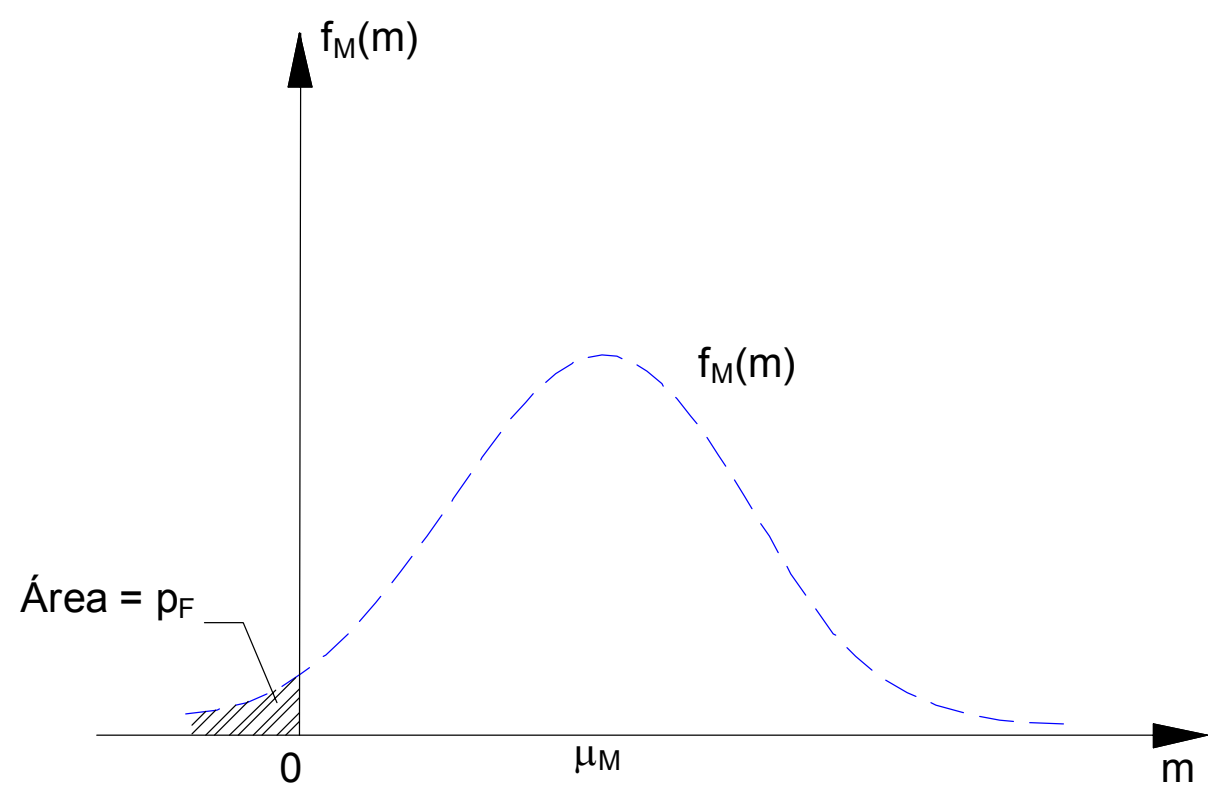

Figura 4.8 - f.d.p. da margem de segurança $M$

Supõe-se a construção de um índice de confiabilidade ou índice de segurança para sistemas estruturais. Evidentemente, deve-se ter informações sobre a variável aleatória $\mathrm{R}$ que corresponde à resistência do sistema $\mathrm{e}$ também sobre a solicitação $Q$. É possível assumir que ambas tenham distribuições Gaussianas, sendo $R \sim N\left(\mu_{R}, \sigma_{R}^{2}\right)$ e $Q \sim N\left(\mu_{Q}, \sigma_{Q}^{2}\right)$.

Dentro dessa suposição, $\mathrm{M}$ será também Gaussiana, pois se trata de uma combinação linear de v.a's Gaussianas, e combinação linear de v.a's Gaussianas é também Gaussiana. Na realidade esta suposição só poderia ser assumida após testes de ajustamento do modelo de probabilidade Gaussiano às amostras de observações das v.a's $\left[r_{1}, r_{2}, \ldots ., r_{n r}\right]$ e $\left[q_{1}, q_{2}, \ldots . ., q_{n q}\right]$. Então, têm-se os parâmetros da v.a. M:

Esperança: $\mu_{M}=E(M)=E[R-Q]=\mu_{R}-\mu_{Q}$,

Variância: $\quad \sigma_{M}^{2}=V(M)=V(R-Q)=V(R)+R(Q)=\sigma_{R}^{2}+\sigma_{Q}^{2}$. 
A colocação da v.a M na forma de Normal Padrão é indicada para que se possa evitar a dependência dos valores dos parâmetros no cálculo das probabilidades na Normal. Assim, a v.a $Z=\left(M-\mu_{M}\right) / \sigma_{M}$ tem distribuição Normal Padrão, ou seja, $Z \sim N(0,1)$. Aplicando estes argumentos, tem-se que:

$$
\begin{gathered}
p_{F}=\int_{-\infty}^{0} f_{M}(m) d m=F_{M}(0)=P(M<0) \\
p_{F}=P\left(\frac{M-\mu_{M}}{\sigma_{M}}<\frac{0-\mu_{M}}{\sigma_{M}}\right)=P\left(Z<\frac{-\mu_{M}}{\sigma_{M}}\right)=\Phi\left(\frac{-\mu_{M}}{\sigma_{M}}\right)
\end{gathered}
$$

e da simetria da curva do modelo Normal, obtém-se a probabilidade de falha:

$$
\mathrm{p}_{\mathrm{F}}=1-\Phi\left(\frac{\mu_{\mathrm{M}}}{\sigma_{\mathrm{M}}}\right)=1-\Phi(\beta)
$$

e, conseqüentemente, tem-se como confiabilidade:

$$
p_{S}=1-p_{F}=\Phi\left(\frac{\mu_{M}}{\sigma_{M}}\right)=\Phi(\beta)=\int_{-\infty}^{\beta} \frac{1}{\sqrt{2 \pi}} e^{-\frac{1}{2} z^{2}} d z
$$

onde

$$
\beta=\frac{\mu_{M}}{\sigma_{M}}=\frac{\mu_{R}-\mu_{Q}}{\sqrt{\sigma_{R}^{2}+\sigma_{Q}^{2}}} .
$$

Assim, é possível construir a Tabela $1 \mathrm{com}$ os valores da probabilidade de falha baseados na diferença das médias das variáveis aleatórias, $\mu_{R}-\mu_{Q}$, e também das medidas de dispersão, $\sigma_{M}^{2}=\sigma_{R}^{2}+\sigma_{Q}^{2}$. 


\begin{tabular}{|c|c|}
\hline $\begin{array}{c}\text { Probabilidade } \\
\text { de falha } \\
\mathrm{P}_{\mathrm{F}}=1-\Phi(\beta)\end{array}$ & \begin{tabular}{c} 
Índice de Confiabilidade \\
\hline 0.50
\end{tabular} \\
\hline 0.25 & 0.67 \\
\hline 0.16 & 1.00 \\
\hline 0.10 & 1.28 \\
\hline 0.05 & 1.65 \\
\hline 0.01 & 2.33 \\
\hline $10^{-3}$ & 3.10 \\
\hline $10^{-4}$ & 3.72 \\
\hline $10^{-5}$ & 4.25 \\
\hline $10^{-6}$ & 4.75 \\
\hline
\end{tabular}

Tabela 4.1- Valores de $\beta$ e da probabilidade de falha $p_{F}$

Os números da Tabela 1 mostram que a confiabilidade do sistema, $\mathrm{p}_{\mathrm{s}}=$ $1-p_{F}$, depende da posição relativa das distribuições $f_{R}(R)$ e $f_{Q}(Q)$ medida pela margem de segurança média $\mu_{M}=\mu_{R}-\mu_{Q}$, e do grau de dispersão das distribuições medido pelo desvio padrão $\sigma_{M}=\sqrt{\sigma_{R}^{2}+\sigma_{Q}^{2}}$.

O índice de confiabilidade $\beta$ é uma função desses dois fatores, sendo que depende diretamente da distância entre os pontos médios das distribuições e inversamente do desvio padrão da variável aleatória correspondente à essa distância. Assim, quanto maior a diferença, $R-Q$, entre a resistência $R$ e a carga $Q$, maior a confiabilidade, $p_{S}=1-p_{F}$, do sistema. $E$, quanto menor as dispersões de $\mathrm{R}$ e de $\mathrm{Q}$, maior será a confiabilidade.

Além da margem de segurança, $\mu_{\mathrm{M}}=\mu_{\mathrm{X}}-\mu_{\mathrm{Y}}$, e do índice de confiabilidade, $\beta=\mu_{M} / \sigma_{M}$, existe também outra estatística (v.a) muito importante nos sistemas estruturais. Trata-se do fator de segurança (FS), definido pela razão entre a capacidade de resistência do sistema e a solicitação, ou seja: 


$$
F S=\frac{X}{Y}
$$

O fator de segurança, $F S=X / Y$, é uma variável aleatória, pois é função de duas variáveis aleatórias. Portanto, possui uma distribuição de probabilidades que pode ser definida por uma f.d.p. Neste caso, a falha é o evento $(F S<1)$ e como probabilidade de falha correspondente, tem-se:

$$
p_{F}=\int_{0}^{1} f_{F S}(\theta) d \theta=F_{F S}(1.0)
$$

como sendo a área hachurada na Figura 4.9.

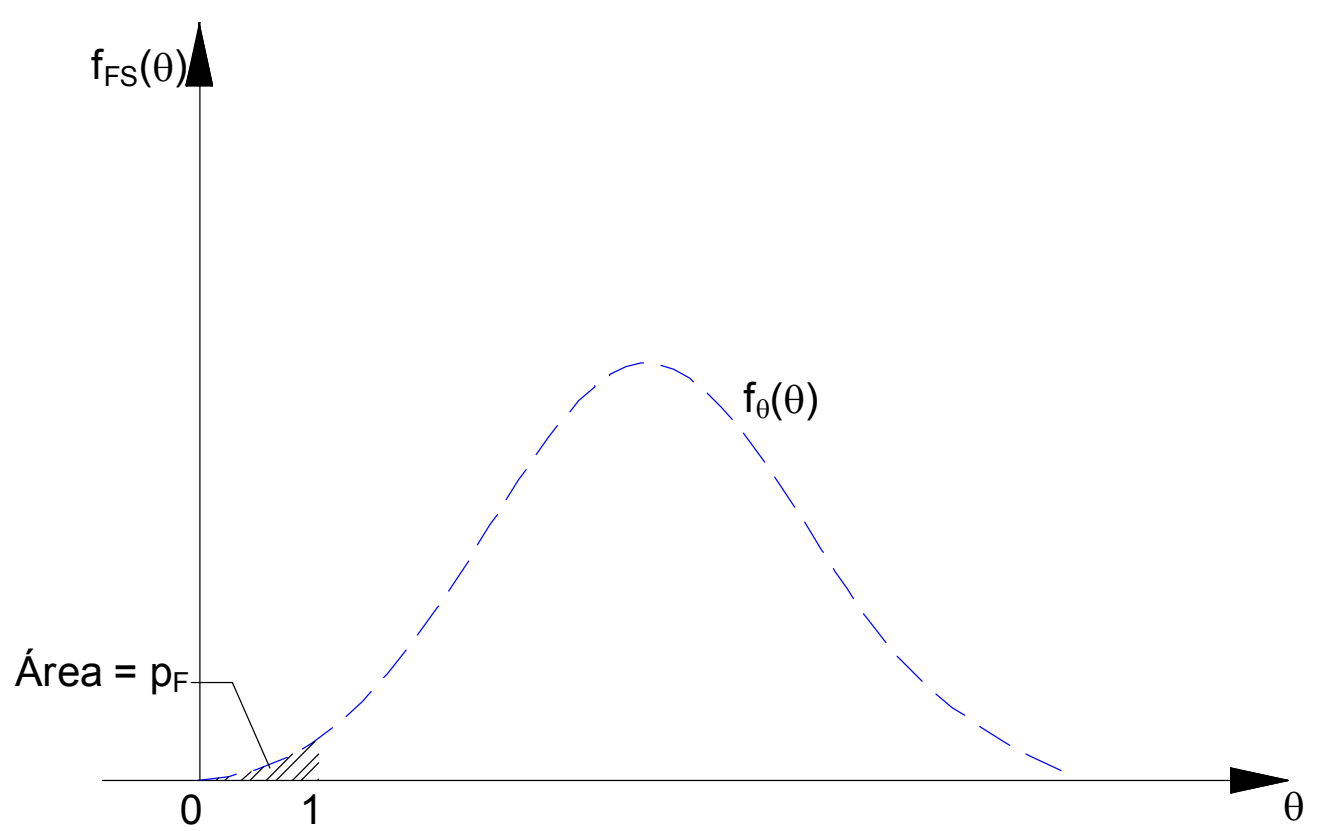

Figura 4.9 - f.d.p. do fator de segurança FS

\subsubsection{Formulação do Segundo Momento}

O cálculo do índice de confiabilidade ou probabilidade de falha necessita do conhecimento das distribuições de probabilidade da capacidade de resistência $X, f_{X}(x)$, e da solicitação $Y, f_{Y}(y)$, ou ainda da distribuição conjunta dessas duas variáveis aleatórias $\mathrm{f}_{\mathrm{X}, \mathrm{Y}}(\mathrm{X}, \mathrm{y})$. 
Na prática, estas distribuições não estão sempre disponíveis em razão da limitação ou inexistência de dados. Outra restrição é os cálculos complicados envolvidos nessa abordagem. Mas a existência de dados permite avaliar os dois primeiros momentos das distribuições. Assim, tem-se disponível a média e o desvio padrão de cada uma das variáveis aleatórias.

Portanto, um índice de confiabilidade, baseado nestas estatísticas, pode ser usado na prática. A confiabilidade pode ser colocada como uma função do primeiro e do segundo momento das variáveis envolvidas no projeto. Por outro lado, quando não se têm informações sobre as formas das distribuições de probabilidade pode-se usar distribuições Normais equivalentes.

Sendo a margem de segurança $M=X-Y$, tem-se que estado de segurança de um sistema estrutural pode ser definido pela condição $(M>0)$; 0 estado de insegurança (falha ou ruptura) é definido por $(M<0)$ e das condições dos dois estados, observa-se que o estado limite é definido por $M=0$.

Tratando as variáveis $\mathrm{X}$ e $\mathrm{Y}$ como Normais Padrões (reduzidas), tem-se:

$$
Z_{X}=\frac{X-\mu_{X}}{\sigma_{X}} \quad \text { e } \quad Z_{Y}=\frac{Y-\mu_{Y}}{\sigma_{Y}} \text {. }
$$

De forma que fazendo a análise no espaço dessas variáveis Normais Padrões, tem-se que o estado limite, $M=X-Y=0$, pode ser representado por:

$$
\begin{gathered}
M=\mu_{X}+\sigma_{X} Z_{X}-\left(\mu_{Y}+\sigma_{Y} Z_{Y}\right)=0 \\
\sigma_{X} Z_{X}-\sigma_{Y} Z_{Y}+\mu_{X}-\mu_{Y}=0 .
\end{gathered}
$$

A função $\sigma_{X} Z_{X}-\sigma_{Y} Z_{Y}+\mu_{X}-\mu_{Y}=0$ é uma reta nas variáveis $Z_{X}$ e $Z_{Y}$. $A$ partir da Figura 4.10, nota-se que distância da reta do estado limite ao ponto da origem do sistema de eixos corresponde a uma medida de confiabilidade. 
Deste modo, calculando-se esta distância, obtém-se uma medida da confiabilidade do sistema.

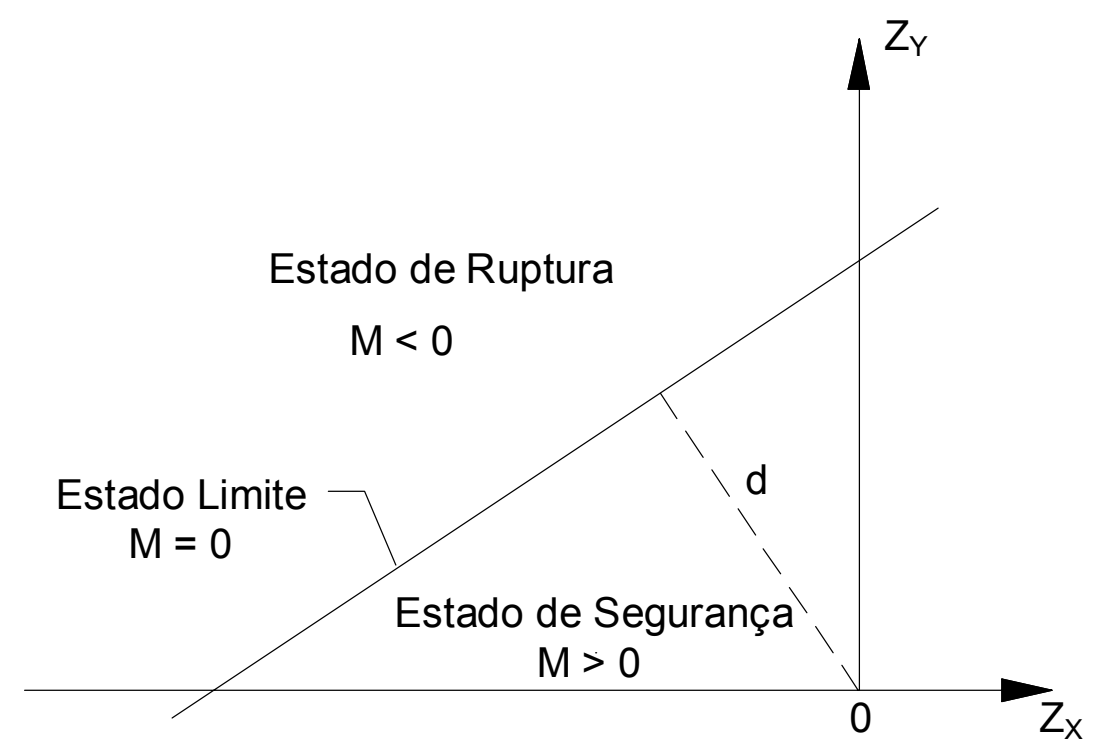

Figura 4.10 - Espaço das variáveis padronizadas $Z_{X}$ e $Z_{Y}$

A confiabilidade de um sistema de engenharia pode envolver várias variáveis. Em particular, a resistência e a solicitação podem ser funções de várias outras variáveis. Para tais casos, o problema resistência-solicitação deve ser generalizado.

Em um amplo sentido, a confiabilidade de um sistema de engenharia pode ser definida como a probabilidade de desempenho de sua função pretendida. O nível de desempenho de um sistema, obviamente, dependerá das propriedades do sistema. Neste contexto, para a proposta de uma formulação generalizada, define-se como uma função desempenho ou função de estado,

$$
g(\underline{X})=g\left(X_{1}, X_{2}, \ldots, X_{n}\right)
$$

onde $\underline{X}=g\left(X_{1}, X_{2}, \ldots, X_{n}\right)$ é um vetor das variáveis de projeto do sistema, e a função $g(\underline{X})$ determina o desempenho ou estado do sistema. Sendo que $g(\underline{X})$ $=0$ é o estado limite do sistema. 
Considerando que

$$
[g(\underline{X})>0]=\text { "estado seguro" }
$$

e

$$
[g(\underline{X})<0]=\text { "estado de falha" }
$$

Geometricamente, a equação do estado limite, $g(\underline{X})=0$, é uma superfície n-dimensional que pode ser chamada de "superfície de falha". Um lado da superfície de falha é o estado seguro, $g(\underline{X})>0$, enquanto que o outro lado da superfície de falha é o próprio estado de falha, $g(\underline{X})<0$.

Portanto, se a f.d.p. conjunta das variáveis de projeto $X_{1}, X_{2}, \ldots, X_{n}$ for $f_{X_{1}, \ldots, x_{n}}\left(x_{1}, \ldots, x_{n}\right)$, a probabilidade do estado de segurança será:

$$
p_{S}=\int_{g(\underline{x})>0} \int f_{x_{1}, \ldots, x_{n}}\left(x_{1}, \ldots, x_{n}\right) d x_{1} \ldots d x_{n}
$$

que pode ser escrita como:

$$
p_{S}=\int_{g(\underline{x})>0} f_{\underline{x}}(\underline{x}) d \underline{x}
$$

A Equação (4.10) é simplesmente a integral de volume de $f_{x}(\underline{x})$ sobre a região segura $g(\underline{X})>0$. De modo inverso, a probabilidade do estado de falha ou probabilidade de falha seria a correspondente integral de volume sobre da região de falha,

$$
p_{S}=\int_{g(\underline{x})<0} f_{\underline{x}}(\underline{x}) d \underline{x} .
$$




\section{Resultado 3}

Seja um sistema estrutural no qual tanto a capacidade de resistência quanto a solicitação de carga são funções de $n$ outras variáveis $X_{1}, X_{2}, X_{3}, \ldots$. , $X_{n}$. Então, a distância do hiperplano do estado limite à origem do sistema de coordenadas reduzidas que mede a confiabilidade do sistema é dada por:

$$
g(\underline{X})=g\left(X_{1}, X_{2}, \ldots, X_{n}\right)=0
$$

\section{PROVA}

Com $n=2$, tem-se como estado limite a reta $\sigma_{X} Z_{X}-\sigma_{Y} Z_{Y}+\mu_{X}-\mu_{Y}=0$ e a distância dessa reta à origem $(0,0)$ é dada, da geometria analítica, por:

$$
d=\frac{\sigma_{X} \cdot 0-\sigma_{Y} \cdot 0+\mu_{X}-\mu_{Y}}{\sqrt{\sigma_{X}^{2}+\sigma_{Y}^{2}}}=\frac{\mu_{X}-\mu_{Y}}{\sqrt{\sigma_{X}^{2}+\sigma_{Y}^{2}}},
$$

que é igual ao índice de confiabilidade $\beta$, já determinado. $E$, generalizando para $\mathrm{n}$ variáveis, $X_{1}, X_{2}, X_{3}, \ldots ., X_{n}$, tem-se que a função que determina $o$ desempenho do sistema é $g\left(X_{1}, X_{2}, X_{3}, \ldots ., X_{n}\right)=g(\underline{X})$, onde $\underline{X}$ é o vetor de estado básico do sistema. $O$ hiperplano do estado limite é $g(\underline{X})=0$ e seguindo a análise com variáveis Normais Padrões tem-se que a distância do ponto $\underline{Z^{\prime}}=\left[Z_{1}, Z_{2}, \ldots, Z_{n}\right]$, na superfície de falha $g(\underline{X})=0$, até a origem de $\underline{Z^{\prime}}$ é:

$$
\mathrm{D}=\sqrt{Z_{X 1}^{2}+Z_{X 2}^{2}+\ldots .+Z_{X n}^{2}}=\left(\underline{Z^{\prime}} \underline{Z}\right)^{1 / 2}
$$

O mais provável ponto de falha tem distância mínima à origem e é obtido minimizando-se $D$, sujeito a restrição $g(\underline{Z})=0$. Aplicando-se o método do multiplicador de Lagrange, $L=D+\lambda g(\underline{X})$, tem-se:

$$
\mathrm{D}=\left(\underline{Z^{\prime}} \underline{\mathrm{Z}}\right)^{1 / 2}+\lambda \mathrm{g}(\underline{\mathrm{X}})
$$




$$
\begin{gathered}
L=\sqrt{Z_{X 1}^{2}+Z_{X 2}^{2}+\ldots+Z_{X n}^{2}}+\lambda g\left(X_{1}, X_{2}, \ldots, X_{n}\right) \\
\frac{\partial L}{\partial Z_{X i}}=\frac{2 Z_{X_{i}}}{2 \sqrt{Z_{X 1}^{2}+Z_{X 2}^{2}+\ldots .+Z_{X n}^{2}}}+\lambda \frac{\partial g}{\partial Z_{X i}}=0 \quad i=1,2, \ldots, n \\
\frac{\partial L}{\partial \lambda}=g\left(X_{1}, X_{2}, \ldots, X_{n}\right)=0
\end{gathered}
$$

A solução do sistema de equações acima, formado por $n+1$ equações, fornece o provável ponto de falha. O vetor gradiente correspondente pode ser escrito como:

$$
\underline{\mathbf{G}}=\left(\frac{\partial \mathrm{g}}{\partial \mathrm{Z}_{\mathrm{X} 1}}, \frac{\partial \mathrm{g}}{\partial \mathrm{Z}_{\mathrm{X} 2}}, \ldots, \frac{\partial \mathrm{g}}{\partial \mathrm{Z}_{\mathrm{Xn}_{\mathrm{n}}}}\right) \quad \text { com } \quad \frac{\partial \mathrm{g}}{\partial \mathrm{Z}_{\mathrm{X}_{\mathrm{i}}}}=\frac{\partial \mathrm{g}}{\partial \mathrm{X}_{\mathrm{i}}} \frac{\mathrm{d} \mathrm{X}_{\mathrm{i}}}{\mathrm{d} \mathrm{Z}_{\mathrm{X}_{\mathrm{i}}}}=\sigma_{\mathrm{X}_{\mathrm{i}}} \frac{\partial \mathrm{g}}{\partial \mathrm{X}_{\mathrm{i}}}
$$

pois

$$
X_{i}=\sigma_{X_{i}} Z_{X_{i}}+\mu_{X_{i}} \quad \text { e } \quad \frac{d X_{i}}{d Z_{X_{i}}}=\sigma_{X_{i}}
$$

Escrevendo a Equação (4.13) na forma matricial, tem-se:

$$
\frac{\underline{Z}}{\left(\underline{Z}^{\prime} \underline{Z}\right)^{1 / 2}}+\lambda \underline{G}=0
$$

mas $(\underline{Z} \underline{Z})^{1 / 2}=\mathrm{D}$, portanto,

$$
\underline{Z}=-\lambda D \underline{G}
$$

e, conseqüentemente, obtém-se:

$$
D=\left[(-\lambda D \underline{G})^{\prime}(-\lambda D \underline{G})\right]^{1 / 2}
$$




$$
\begin{aligned}
& D=\lambda D\left(\underline{G}^{\prime} \underline{G}\right)^{1 / 2} \\
& \lambda=\left(\underline{G}^{\prime} \underline{G}\right)^{-1 / 2}
\end{aligned}
$$

logo,

$$
\begin{aligned}
& \underline{Z}=-\lambda D \underline{G}=-D \cdot\left(\underline{G^{\prime}} \underline{G}\right)^{-1 / 2} \cdot \underline{G} \\
& \underline{Z}=-\frac{\underline{G}^{\prime} D}{\left(\underline{G}^{\prime} \underline{G}\right)^{1 / 2}} .
\end{aligned}
$$

Multiplicando-se à esquerda por $\underline{G}^{\prime}$ os dois membros, tem-se:

$$
\begin{aligned}
& \underline{G}^{\prime} \underline{Z}=-D\left(\underline{G}^{\prime} \underline{G}\right)^{-1 / 2} \cdot \underline{G}^{\prime} \underline{G} \\
& \underline{G}^{\prime} \underline{Z}=-D\left(\underline{G}^{\prime} \underline{G}\right)^{1 / 2} \\
& D=-\frac{\underline{G}^{\prime} \underline{Z}}{\left(\underline{G}^{\prime} \underline{G}\right)^{1 / 2}}
\end{aligned}
$$

Portanto, substituindo a Equação (4.16) em (4.14), obtém-se o valor da distância mínima:

$$
d_{\min }=-\frac{\underline{G}^{* \prime} \underline{Z}^{*}}{\left(\underline{G}^{* \prime} \underline{G}^{*}\right)^{1 / 2}}=\beta,
$$

onde $\underline{G}^{*}$ é o vetor gradiente no mais provável ponto de falha $\left[z_{x 1}^{*}, z_{x 2}^{*}, \ldots \ldots\right.$, $z_{X_{n}}^{*}$ ] e na forma escalar, tem-se: 


$$
\beta=-\frac{\sum_{i=1}^{n} \underline{z}_{x_{i}}^{*}\left(\frac{\partial g}{\partial \underline{z}_{x_{i}}^{*}}\right)}{\sqrt{\sum_{i=1}^{n}\left(\frac{\partial g}{\partial \underline{z}_{x_{i}}^{*}}\right)^{2}}}
$$

Aplicando $\beta$ na Equação (4.17), o mais provável ponto de falha torna-se:

$$
\underline{Z}^{*}=-\frac{G^{*} d_{\min }}{\left(\underline{G}^{* \prime} \underline{G}^{*}\right)^{1 / 2}}
$$

\section{Resultado 4}

Seja $g(\underline{X})$ uma variável aleatória função de estado de um sistema estrutural. Então a razão entre a média de $g(\underline{X})$ e o desvio padrão de $g(\underline{X})$ é igual à distância do plano tangente à superfície de falha em $Z^{*}$ à origem do sistema de coordenadas em variáveis reduzidas.

\section{PROVA}

Desenvolvendo a função $\mathrm{g}(\underline{\mathrm{X}})$ em série de Taylor no ponto $\underline{x}^{*}$, situado na superfície do estado limite $g\left(\underline{x}^{*}\right)=0$, tem-se:

$$
\begin{aligned}
& g\left(X_{1}, X_{2}, \ldots, X_{n}\right)=g\left(x_{1}^{*}, \quad x_{2}^{*}, \quad \ldots ., \quad x_{n}^{*}\right)+\sum_{i=1}^{n}\left(X_{i}-x_{i}^{*}\right) \frac{\partial g}{\partial X_{i}^{*}}+ \\
& \frac{\sum_{j=1}^{n} \sum_{i=1}^{n}\left(X_{i}-X_{i}^{*}\right)\left(X_{j}-X_{j}^{*}\right)}{\frac{\partial^{2} g}{\partial X_{i}^{*} \partial X_{j}^{*}}}+\ldots
\end{aligned}
$$

e desde que $g\left(x_{1}^{*}, x_{2}^{*}, \ldots ., x_{n}^{*}\right)=0$, 
$g\left(X_{1}, X_{2}, \ldots, X_{n}\right)=\sum_{i=1}^{n}\left(X_{i}-X_{i}^{*}\right) \frac{\partial g}{\partial X_{i}^{*}}+\frac{\sum_{j=1}^{n} \sum_{i=1}^{n}\left(X_{i}-X_{i}^{*}\right)\left(X_{j}-X_{j}^{*}\right)}{\frac{\partial^{2} g}{\partial X_{i}^{*} \partial X_{j}^{*}}}+\ldots$

mas escrevendo em variáveis reduzidas $\frac{X_{i}-\mu_{X_{i}}}{\sigma_{X_{i}}}=Z_{i}$ e $\frac{X_{i}^{*}-\mu_{X_{i}}}{\sigma_{X_{i}}}=z_{i}^{*}$, tem-se:

$$
X_{i}-X_{i}^{*}=\left(\sigma_{X_{i}} Z_{i}+\mu_{X_{i}}\right)-\left(\sigma_{X_{i}} Z_{i}^{*}+\mu_{X_{i}}\right)=\sigma_{X_{i}}\left(Z_{i}-Z_{i}^{*}\right)
$$

$$
\frac{\partial g}{\partial Z_{X_{i}}}=\frac{\partial g}{\partial X_{i}} \frac{\partial X_{i}}{\partial Z_{X_{i}}}=\sigma_{X_{i}} \frac{\partial g}{\partial X_{i}}
$$

$$
\frac{\partial g}{\partial X_{i}}=\frac{\partial g}{\partial Z_{X_{i}}} \frac{\partial Z_{X_{i}}}{\partial X_{i}}=\frac{1}{\sigma_{X_{i}}} \frac{\partial g}{\partial Z_{X_{i}}}
$$

pois

$$
\frac{\partial \mathbf{X}_{i}}{\partial Z_{X_{i}}}=\frac{\partial\left(\sigma_{X_{i}} Z_{i}+\mu_{X_{i}}\right)}{\partial Z_{X_{i}}}=\sigma_{X_{i}}
$$

então:

$$
g\left(X_{1}, X_{2}, \ldots, X_{n}\right)=\sum_{i=1}^{n} \sigma_{X_{i}}\left(Z_{X_{i}}-Z_{X_{i}}^{*}\right) \frac{1}{\sigma_{X_{i}}} \frac{\partial g}{\partial Z_{X_{i}}^{*}}+\ldots=\sum_{i=1}^{n}\left(Z_{X_{i}}-Z_{X_{i}}^{*}\right) \frac{\partial g}{\partial Z_{X_{i}}^{*}}+\ldots
$$

e truncando a série para uma aproximação de primeira ordem e calculando-se $E(g)$ e $V(g)$, tem-se:

$$
E(g) \cong \mu_{g} \cong E\left(\sum_{i=1}^{n}\left(Z_{X_{i}}-z_{X_{i}}^{*}\right) \frac{\partial g}{\partial Z_{X_{i}}^{*}}+\ldots\right)=\sum_{i=1}^{n} E\left(Z_{X_{i}}-Z_{X_{i}}^{*}\right) \frac{\partial g}{\partial Z_{X_{i}}^{*}}+\ldots
$$




$$
\begin{aligned}
& E(g) \cong \mu_{g} \cong \sum_{i=1}^{n}\left(0-z_{x_{i}}^{*}\right) \frac{\partial g}{\partial Z_{X_{i}}^{*}}+\ldots=-\sum_{i=1}^{n} z_{X_{i}}^{*} \frac{\partial g}{\partial Z_{X_{i}}^{*}} \\
& V(g) \cong \sigma_{g}^{2} \cong V\left[\sum_{i=1}^{n}\left(Z_{x_{i}}-z_{X_{i}}^{*}\right) \frac{\partial g}{\partial Z_{X_{i}}^{*}}+\ldots\right]=\sum_{i=1}^{n} V\left[\left(Z_{X_{i}}-z_{X_{i}}^{*}\right) \frac{\partial g}{\partial Z_{X_{i}}^{*}}+\ldots\right] \\
& \left.V(g) \cong \sigma_{g}^{2} \cong \sum_{i=1}^{n}\left(V\left(Z_{x_{i}}\right)-0\right)\right)\left(\frac{\partial g}{\partial Z_{x_{i}}^{*}}\right)^{2} \\
& \left.V(g) \cong \sigma_{g}^{2} \cong \sum_{i=1}^{n}(1-0)\right)\left(\frac{\partial g}{\partial Z_{x_{i}}^{*}}\right)^{2} \\
& V(g) \cong \sigma_{g}^{2} \cong \sum_{i=1}^{n}\left(\frac{\partial g}{\partial Z_{x_{1}}}\right)^{2}
\end{aligned}
$$
idêntica a expressão encontrada para a distância mínima no Resultado 3.

\subsubsection{Funções de Desempenho Lineares}

Considerando-se uma classe de funções de desempenho lineares, com exceção de suas próprias utilidades, certos aspectos de casos lineares seriam a base para uma aproximação de funções de desempenho não-lineares.

Uma função de desempenho linear pode ser representada como:

$$
g(\underline{X})=a_{0}+\sum_{i} a_{i} X_{i},
$$

onde $a_{0}$ e $a_{i}$ são constantes. A correspondente equação do estado limite pode ser escrita da forma:

$$
a_{0}+\sum_{i} a_{i} x_{i}=0
$$


Em termos de variáveis padronizadas, a equação do estado limite tornase:

$$
a_{0}+\sum_{i} a_{i}\left(\sigma_{X_{i}} Z_{i}+\mu_{X_{i}}\right)=0
$$

Em três dimensões a equação acima pode ser escrita como:

$$
a_{0}+a_{1}\left(\sigma_{X_{1}} Z_{1}+\mu_{X_{1}}\right)+a_{2}\left(\sigma_{X_{2}} Z_{2}+\mu_{X_{2}}\right)+a_{3}\left(\sigma_{X_{3}} Z_{3}+\mu_{X_{3}}\right)=0
$$

que é uma superfície plana no espaço $z_{\times 1}, z_{\times 2}, z_{\times 3}$ como mostra a Figura 4.11.

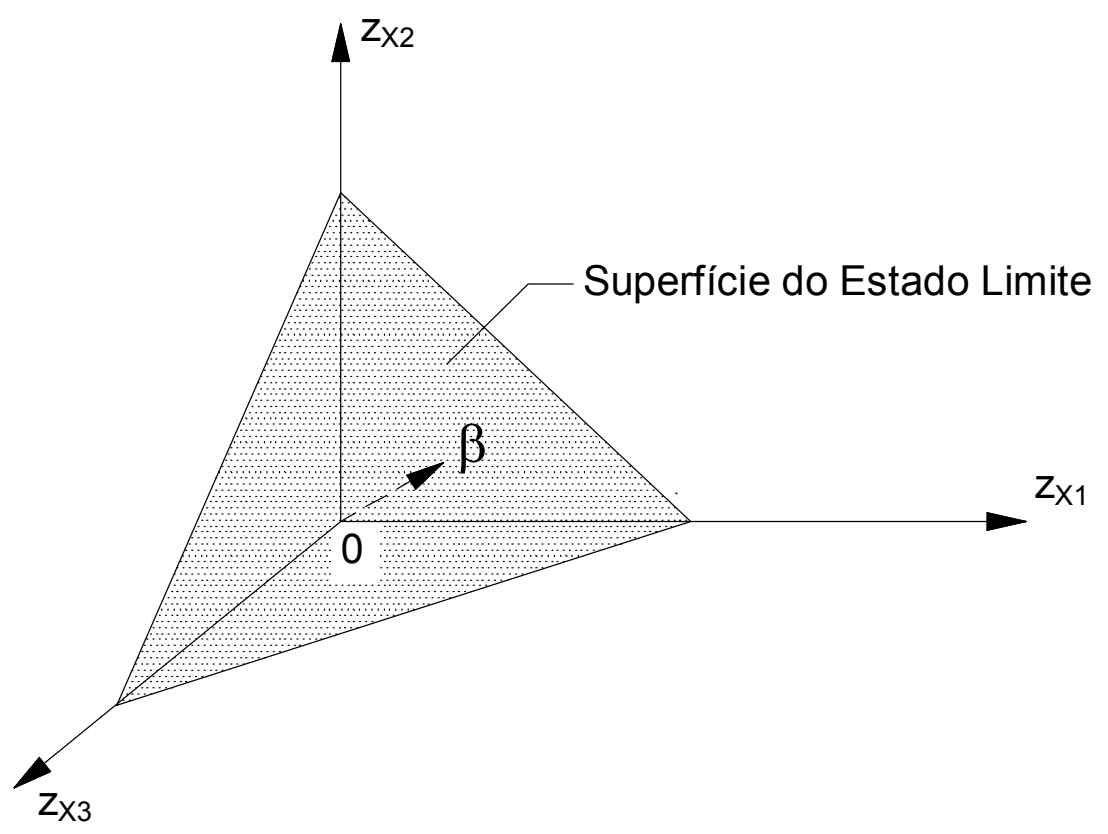

Figura 4.11 - Superfície linear do estado limite no espaço $z_{x 1}, z_{x 2}, z_{x 3}$

Sendo

$$
g(\underline{X})=a_{0}+\sum_{i} a_{i} X_{i}
$$

e sabendo-se que 


$$
\begin{gathered}
\mu_{g}=E(g(\underline{X}))=E\left(a_{0}+\sum_{i=1}^{n} a_{i} X_{i}\right) \\
\mu_{g}=a_{0}+\sum_{i=1}^{n} a_{i} E\left(X_{i}\right) \\
\mu_{g}=a_{0}+\sum_{i=1}^{n} a_{i} \mu_{X_{i}}
\end{gathered}
$$

e

$$
\begin{gathered}
V(g)=V(g(\underline{X}))=V\left(a_{0}+\sum_{i=1}^{n} a_{i} X_{i}\right) \\
V(g)=0+\sum_{i=1}^{n} a_{i}{ }^{2} V\left(X_{i}\right) \\
V(g)=\sum_{i=1}^{n} a_{i}{ }^{2} \sigma_{X_{i}}^{2}
\end{gathered}
$$

e

$$
\sigma_{g}=\sqrt{V(g)}=\sqrt{\sum_{i=1}^{n} a_{i}{ }^{2} \sigma_{X_{i}}^{2}},
$$

tem-se:

$$
\beta=\frac{\mu_{g}}{\sigma_{g}}
$$

Portanto, tem-se como distância do plano de falha à origem das variáveis padronizadas $\mathrm{Z}$ :

$$
\beta=\frac{a_{0}+\sum_{i} a_{i} \mu_{x_{i}}}{\sqrt{\sum_{i}\left(a_{i} \sigma_{X_{i}}\right)^{2}}}
$$


A equação acima pode também ser obtida diretamente da Equação (4.19). Nota-se que para duas variáveis normais não correlacionadas, $X$ e $Y$, a probabilidade do estado de segurança, $p_{S}$, é diretamente uma função da distância da linha de falha à origem das variáveis normalizadas; isto é, a distância $\beta$. Este resultado pode ser generalizado, ou seja, se as variáveis aleatórias $X_{1}, X_{2}, \ldots, X_{n}$ são variáveis normais não correlacionadas, a probabilidade do estado de segurança no presente caso é:

$$
\begin{aligned}
& p_{S}=P\left(a_{0}+\sum_{i=1}^{n} a_{i} X_{i}>0\right) \\
& p_{S}=P\left(\frac{a_{0}+\sum_{i=1}^{n} a_{i} X_{i}-a_{0}-\sum_{i=1}^{n} a_{i} \mu_{X_{i}}}{\sqrt{\sum_{i=1}^{n}\left(a_{i}{ }^{2} \sigma_{X_{i}}\right)^{2}}}>\frac{0-a_{0}-\sum_{i=1}^{n} a_{i} \mu_{X_{i}}}{\sqrt{\sum_{i=1}^{n}\left(a_{i}{ }^{2} \sigma_{X_{i}}\right)^{2}}}\right) \\
& p_{S}=P\left(Z>\frac{-a_{0}-\sum_{i=1}^{n} a_{i} \mu_{X_{i}}}{\sqrt{\sum_{i=1}^{n}\left(a_{i}{ }^{2} \sigma_{X_{i}}\right)^{2}}}\right)=1-\Phi\left[\frac{-\left(a_{0}+\sum_{i=1}^{n} a_{i} \mu_{X_{i}}\right)}{\sqrt{\sum_{i=1}^{n}\left(a_{i}{ }^{2} \sigma_{X_{i}}\right)^{2}}}\right] \\
& p_{S}=\Phi\left[\frac{a_{0}+\sum_{i=1}^{n} a_{i} \mu_{X_{i}}}{\sqrt{\sum_{i=1}^{n}\left(a_{i}{ }^{2} \sigma_{X_{i}}\right)^{2}}}\right]
\end{aligned}
$$

Comparando as Equações (4.21) e (4.22), o argumento dentro dos colchetes da Equação (4.22) é a distância $\beta$. Conseqüentemente, a probabilidade $p_{s}$ é novamente uma função da distância do plano de ruptura $g(\underline{X})=0$ à origem da variáveis normalizadas. No caso geral de $n$ variáveis normais não correlacionadas, a probabilidade de segurança é

$$
\mathrm{p}_{\mathrm{S}}=\Phi(\beta)
$$




\section{MÉTODO DE SUPERFÍCIE DE RESPOSTA}

\subsection{Introdução}

Muito do que se conhece em engenharia é desenvolvido através de testes ou experiências. Geralmente, engenheiros trabalham em áreas em que nenhuma teoria de engenharia é completamente aplicável. Assim, a experiência e a observação dos dados resultantes constituem as únicas maneiras pelas quais o problema pode ser resolvido.

Mesmo que haja uma boa teoria científica em que se pode confiar na explicação do fenômeno de interesse, é quase sempre necessário conduzir testes ou experimentos para confirmar se a teoria é, na verdade, aplicável na situação ou no ambiente no qual está sendo empregada. Julgamentos estatísticos e métodos estatísticos desempenham um papel importante no planejamento, condução e análise de dados a partir de experimentos de engenharia.

Um experimento é somente um teste ou uma série de testes. Experimentos são feitos em todas as áreas de engenharia e são importantes na maneira de se aprender como sistemas e processos funcionam. A validade das conclusões retiradas de um experimento depende em grande extensão de como o experimento foi conduzido. Conseqüentemente, o planejamento do experimento desenvolve o papel principal na solução futura do problema que inicialmente motivou o experimento. 


\subsection{Experimentos Fatoriais}

Quando vários fatores são importantes, a melhor estratégia da experiência é planejar algum tipo de experimento fatorial. Um experimento fatorial é aquele em que os fatores são variados conjuntamente.

\subsubsection{Experimento de Otimização}

Em um experimento de caracterização, deve-se determinar que fatores afetam a resposta. A próxima etapa é determinar a região nos fatores importantes que conduz a uma resposta ótima. Por exemplo, se a resposta for dispendiosa, procura-se por uma região de custo mínimo.

\subsection{Planejamento de Experimentos}

Quando vários fatores são de interesse em um experimento, um planejamento fatorial de experimentos deve ser usado. Como notado previamente, os fatores são variados conjuntamente nesses experimentos.

Por um planejamento fatorial, pode-se dizer que, em cada tentativa completa ou réplica de experimento, todas as combinações possíveis dos níveis dos fatores são investigadas.

\subsubsection{Aplicação das Técnicas de Planejamento de Experimentos}

O planejamento de experimentos é uma ferramenta extremamente importante no interesse de se melhorar o desempenho de um determinado sistema. Também tem uma extensiva aplicação no desenvolvimento de novos processos e no planejamento de novos produtos. 


\subsubsection{Planejamentos Fatoriais}

Planejamentos fatoriais são freqüentemente usados nos experimentos envolvendo vários fatores em que é necessário estudar o efeito conjunto dos fatores sobre uma resposta. Entretanto, vários casos especiais do planejamento fatorial geral são importantes pelo fato deles serem largamente empregados em trabalhos de pesquisa e devido ao fato deles formarem a base de outros planejamentos de considerável valor prático.

O mais importante desses casos especiais é aquele de $\mathrm{k}$ fatores, cada um com somente dois níveis. Esses níveis podem ser quantitativos, tais como os valores da resistência do concreto e do aço, ou eles podem ser qualitativos tais como os níveis "alto" ou "baixo" de um fator, ou talvez a presença e a ausência de um fator. Uma réplica completa de tal planejamento requer $2 \times 2 \times$ $2 \times 2 \times \ldots \times 2=2^{\mathrm{k}}$ observações, sendo chamada planejamento fatorial $2^{\mathrm{k}}$.

O planejamento $2^{k}$ é, particularmente, útil nos estágios iniciais de um trabalho experimental quando muitos fatores são prováveis de serem investigados. Ele fornece o menor número de corridas para as quais os $\mathrm{k}$ fatores podem ser estudados em um planejamento fatorial completo. Porque há somente dois níveis de cada fator, temos de supor que a resposta seja aproximadamente linear na faixa dos níveis dos fatores escolhidos.

\subsection{Métodos e Planejamentos de Superfície de Resposta}

A metodologia da superfície de resposta (MSR) é uma coleção de técnicas matemáticas e estatísticas que são úteis para modelagem e análise nas aplicações em que a resposta de interesse seja influenciada por várias variáveis e o objetivo seja otimizar essa resposta. Por exemplo, deseja-se encontrar as resistências do aço $\left(x_{1}\right)$ e do concreto $\left(x_{2}\right)$ que maximizem a capacidade $(\mathrm{Y})$ de uma estrutura de concreto armado. A capacidade estrutural é uma função das resistências do aço e do concreto, como: 


$$
Y=f\left(x_{1}, x_{2}\right)+\epsilon
$$

em que $\in$ representa o ruído ou erro observado na resposta $Y$. Denotando-se a resposta esperada por $E(Y)=f\left(x_{1}, x_{2}\right)=\eta$, pois

$$
\begin{gathered}
Y=f\left(x_{1}, x_{2}\right)+\epsilon, \quad \text { onde } \in \sim \mathcal{N}\left(0, \sigma^{2}\right) . \\
E(Y)=E\left(f\left(x_{1}, x_{2}\right)+\epsilon\right)=E\left(f\left(x_{1}, x_{2}\right)\right)+E(\epsilon)=f\left(x_{1}, x_{2}\right)+0 \\
E(Y)=f\left(x_{1}, x_{2}\right)=\eta,
\end{gathered}
$$

então a superfície representada por

$$
\eta=f\left(x_{1}, x_{2}\right)
$$

é chamada de superfície de resposta.

Pode-se representar a superfície de resposta como um gráfico de superfície em um espaço tridimensional. Com o objetivo de visualizar a forma de uma superfície de resposta, geralmente, plota-se os contornos da superfície de resposta.

No gráfico dos contornos, conhecido como gráfico das curvas de nível, linhas de resposta constantes são desenhadas no plano $\mathrm{x}_{1}, \mathrm{x}_{2}$. Cada contorno corresponde a uma altura particular da superfície de resposta. O gráfico das curvas de nível é útil no estudo dos níveis de $x_{1}$ e $x_{2}$ que resultam nas mudanças na forma ou na altura da superfície de resposta.

Na maioria dos problemas de MSR, a forma da relação entre a resposta e as variáveis independentes é desconhecida. Assim, a primeira etapa na MSR é encontrar uma aproximação adequada para a relação verdadeira entre $Y$ e as variáveis independentes. Geralmente, emprega-se um polinômio de baixo grau em alguma região das variáveis independentes. Se a resposta for bem 
modelada por uma função linear das variáveis independentes, então a função aproximadora será o modelo de primeira ordem:

$$
Y=\beta_{0}+\beta_{1} x_{1}+\beta_{2} x_{2}+\ldots+\beta_{k} x_{k}+\epsilon
$$

Se houver curvatura no sistema, então um polinomial de maior grau tem de ser usado, tal como o modelo de segunda ordem:

$$
Y=\beta_{0}+\sum_{i=1}^{k} \beta_{i} x_{i}+\sum_{i=1}^{k} \beta_{i i} x_{i}^{2}+\sum_{i} \sum_{\substack{j \\ i<j}} \beta_{i j} x_{i} x_{j}+\epsilon .
$$

Muitos problemas de MSR utilizam uma ou ambas dessas aproximações polinomiais. Naturalmente, é improvável que um modelo polinomial seja uma aproximação razoável da relação funcional verdadeira sobre o espaço inteiro das variáveis independentes, porém para uma região relativamente pequena, geralmente, funcionarão muito bem.

O método dos mínimos quadrados é usado para estimar os parâmetros nas aproximações polinomiais. A análise de superfície de resposta é então feita em termos da superfície ajustada. Se a superfície ajustada for uma aproximação adequada da função verdadeira de resposta, então a análise da superfície ajustada será aproximadamente equivalente à análise do sistema real.

MSR é um procedimento seqüencial. Freqüentemente, quando se estiver em um ponto na superfície de resposta longe do ótimo, tal como as condições operacionais atuais, há pouca curvatura no sistema e o modelo de primeira ordem será apropriado. O objetivo é chegar rápido e eficientemente à vizinhança geral do ótimo. Uma vez em que a região do ótimo tenha sido encontrada, um modelo mais elaborado, tal como o modelo de segunda ordem, pode ser empregado e uma análise pode ser feita para localizar o máximo. 
O objetivo final do MSR é determinar as condições operacionais ótimas para o sistema ou determinar uma região do espaço fatorial, em que as especificações operacionais sejam satisfeitas. Nota-se também que a palavra ótimo na MSR é usada em um sentido especial. Os procedimentos da MSR garantem convergência para somente um ótimo local.

\subsubsection{Método da Ascendente de Maior Inclinação (Steepest Ascent)}

Usualmente, a estimativa inicial das condições operacionais ótimas para o sistema estará longe do ótimo real. Em tais circunstâncias, o objetivo do experimentalista é mover rapidamente em direção à vizinhança geral do ótimo. Deseja-se usar um procedimento experimental simples e eficiente economicamente. Quando se estiver longe do ótimo, geralmente, considera-se um modelo de primeira ordem como uma aproximação adequada da superfície verdadeira em uma região pequena dos x's.

O método da ascendente de maior inclinação é um procedimento para se mover seqüencialmente ao longo do caminho ascendente de maior inclinação, ou seja, na direção de aumento máximo na resposta. Naturalmente, se a minimização for desejada, então se fala sobre o método da descendente de maior inclinação. O modelo ajustado de primeira ordem é

$$
\hat{y}=\hat{\beta}_{0}+\sum_{i=1}^{k} \hat{\beta}_{i} x_{i}
$$

e a superfície de resposta de primeira ordem, isto é, os contornos de $\hat{y}$, é uma série de linhas paralelas. A direção da ascendente de maior inclinação é a direção em que $\hat{y}$ cresce mais rapidamente. Essa direção é normal aos contornos da superfície ajustada de resposta.

Geralmente, tomamos como o caminho ascendente de maior inclinação a linha que passa através do centro da região de interesse e que seja normal 
aos contornos da superfície ajustada. Logo, as etapas ao longo do caminho são proporcionais aos coeficientes de regressão $\left\{\hat{\beta}_{i}\right\}$. O experimentalista determina o tamanho real da etapa, baseado no conhecimento do processo ou em outras considerações práticas.

Os experimentos são conduzidos ao longo do caminho ascendente de maior inclinação até que mais nenhum aumento seja observado na resposta. Então um novo modelo de primeira ordem pode ser usado, uma nova direção da ascendente de maior inclinação é determinada e mais experimentos são conduzidos naquela direção, até que o experimentalista sinta que o processo está próximo do ótimo.

\subsubsection{Análise de uma Superfície de Resposta de Segunda Ordem}

Quando o experimentalista estiver relativamente próximo do ótimo, um modelo de segunda ordem é geralmente utilizado para aproximar a resposta por causa da curvatura na verdadeira superfície de resposta. O modelo ajustado de segunda ordem é

$$
\hat{y}=\hat{\beta}_{0}+\sum_{i=1}^{k} \hat{\beta}_{i} x_{i}+\sum_{i=1}^{k} \hat{\beta}_{i i} x_{i}^{2}+\sum_{i} \sum_{\substack{j \\ i<j}} \hat{\beta}_{i j} x_{i} x_{j}
$$

em que $\hat{\beta}$ denota a estimativa de mínimos quadrados de $\beta$.

\subsubsection{Modelo de Regressão Linear Múltipla}

Muitas aplicações da análise de regressão envolvem situações em que há mais de um regressor $x$ e uma variável dependente ou variável de resposta Y. Um modelo de regressão que contenha mais de um regressor é chamado de um modelo de regressão múltipla. 


$$
\mathrm{Y}=\beta_{0}+\beta_{1} \mathrm{x}_{1}+\beta_{2} \mathrm{x}_{2}+\epsilon
$$

A Equação (5.4) mostra um modelo de regressão linear múltipla com dois regressores. O termo linear é usado porque a Equação (5.4) é uma função linear dos parâmetros desconhecidos $\beta_{0}, \beta_{1}$ e $\beta_{2}$. O modelo de regressão descreve um plano no espaço tridimensional de $\mathrm{Y}, \mathrm{x}_{1}$ e $\mathrm{x}_{2}$ conforme mostrado pela Figura 5.1. O parâmetro $\beta_{0}$ é a interseção do plano, $\beta_{1}$ e $\beta_{2}$ chamam-se coeficientes parciais de regressão porque $\beta_{1}$ mede a variação esperada em $Y$ por unidade de variação em $x_{1}$ quando $x_{2}$ for constante, e $\beta_{2}$ mede a variação esperada em $\mathrm{Y}$ por unidade de variação em $\mathrm{x}_{2}$ quando $\mathrm{x}_{1}$ for constante.

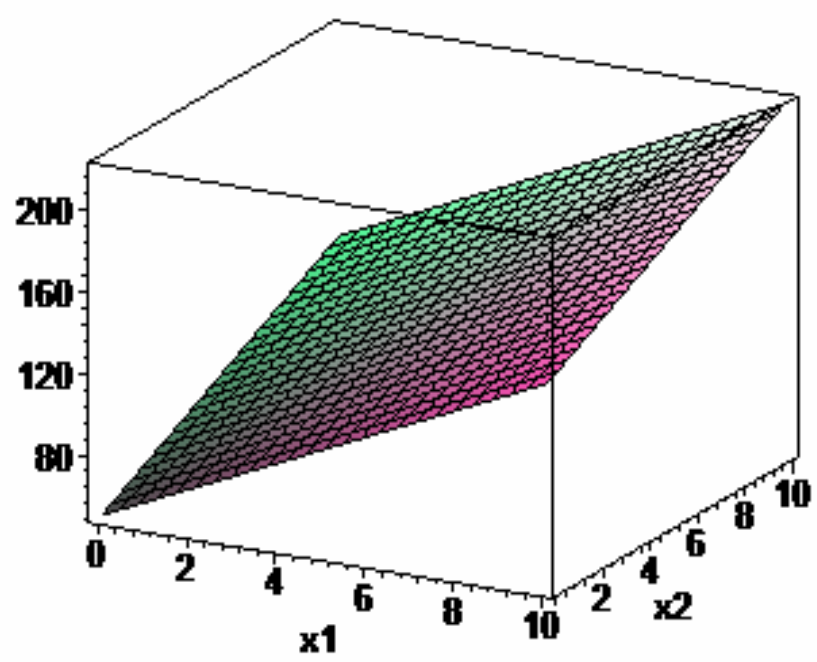

Figura 5.1 - Exemplo de um plano de regressão

A Figura 5.2 mostra uma curva de nível do modelo de regressão, ou seja, linhas de $E(Y)$ constante, como uma função de $x_{1}$ e $x_{2}$. Nota-se que as linhas de nível são retas. 


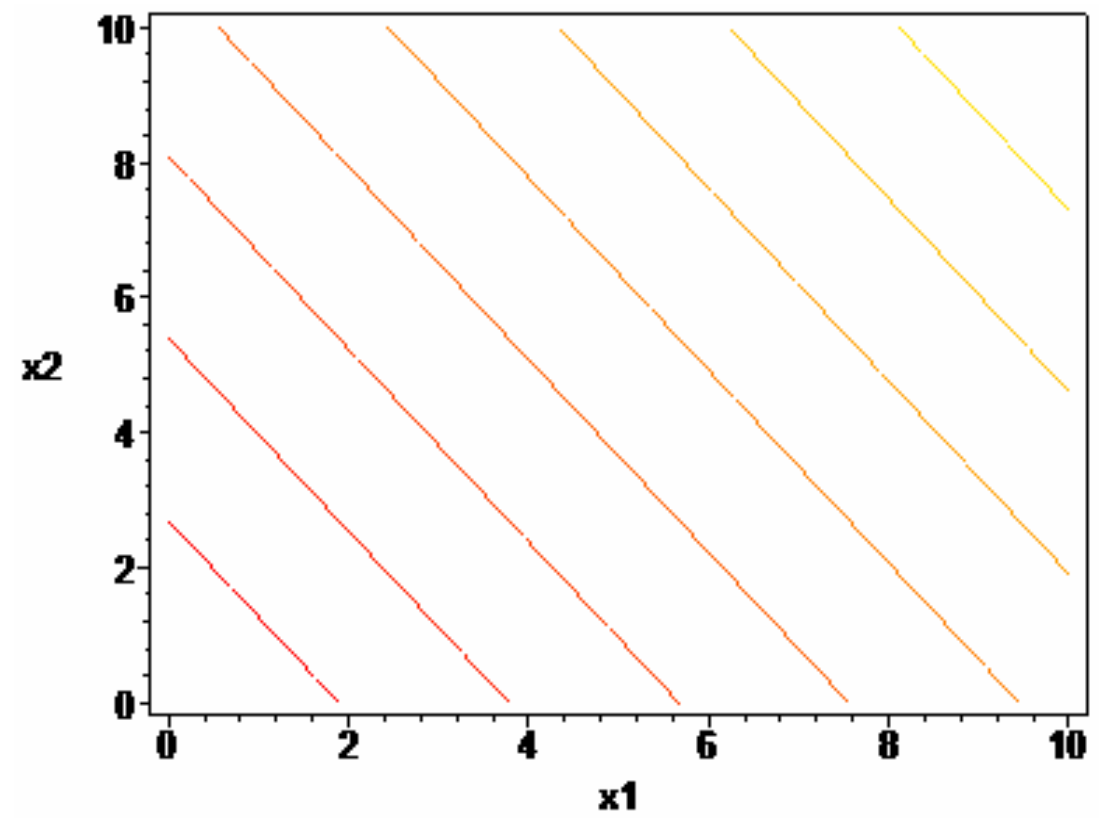

Figura 5.2 - Exemplo de curvas de nível

Em geral, a variável dependente ou de resposta $Y$ pode ser relacionada a $\mathrm{k}$ variáveis independentes ou regressores. O modelo

$$
\mathrm{Y}=\beta_{0}+\beta_{1} \mathrm{x}_{1}+\beta_{2} \mathrm{x}_{2}+\ldots+\beta_{\mathrm{k}} \mathrm{x}_{\mathrm{k}}+\epsilon
$$

é chamado de modelo de regressão linear múltipla com $\mathrm{k}$ regressores. Os parâmetros $\beta_{\mathrm{j}}, \mathrm{j}=0,1, \ldots, k$, são chamados de coeficientes de regressão. Esse modelo descreve um hiperplano no espaço $k$-dimensional dos regressores $\left\{X_{j}\right\}$. O parâmetro $\beta_{\mathrm{j}}$ representa a variação esperada na resposta $Y$ por unidade de variação unitária em $x_{j}$ quando todos os outros regressores $x_{i}(i \neq j)$ forem mantidos constantes.

Modelos de regressão linear múltipla são freqüentemente usados como aproximações de funções. Isto é, a verdadeira relação funcional entre $Y$ e $x_{1}$, $\mathrm{x}_{2}, \ldots, \mathrm{x}_{\mathrm{k}}$ é desconhecida, porém em certas faixas das variáveis independentes, o modelo de regressão linear será uma aproximação adequada.

Modelos que sejam mais complexos na estrutura do que a Equação (5.5) podem ainda ser analisados por técnicas de regressão múltipla. Por exemplo, considerando-se o modelo polinomial cúbico com um regressor. 


$$
Y=\beta_{0}+\beta_{1} x+\beta_{2} x^{2}+\beta_{3} x^{3}+\epsilon
$$

Fazendo-se $x_{1}=x, x_{2}=x^{2}, x_{3}=x^{3}$, então a Equação (5.6) pode ser escrita como:

$$
Y=\beta_{0}+\beta_{1} x_{1}+\beta_{2} x_{2}+\beta_{3} x_{3}+\epsilon
$$

que é modelo de regressão múltipla com três regressores.

Modelos que incluem efeitos de interação podem ser analisados pelos métodos de regressão múltipla. Uma interação entre duas variáveis pode ser representada por um termo cruzado no modelo, tal como

$$
Y=\beta_{0}+\beta_{1} x_{1}+\beta_{2} x_{2}+\beta_{12} x_{1} x_{2}+\in
$$

Fazendo-se $x_{3}=x_{1} x_{2}$ e $\beta_{3}=\beta_{12} x_{1} x_{2}$, então a Equação (5.8) pode ser escrita como

$$
Y=\beta_{0}+\beta_{1} x_{1}+\beta_{2} x_{2}+\beta_{3} x_{3}+\epsilon
$$

que é um modelo de regressão linear múltipla.

As Figuras 5.3 e 5.4 mostram o gráfico tridimensional de um modelo de regressão e as curvas de nível bidimensionais correspondentes. Observa-se que, embora esse seja um modelo de regressão linear, a forma da superfície que é gerada pelo modelo não é linear. Em geral, qualquer modelo de regressão que seja linear nos parâmetros (os $\beta$ 's) é um modelo de regressão linear, independente da forma da superfície gerada.

As Figuras 5.3 e 5.4 fornecem uma boa interpretação gráfica de uma interação. Geralmente, a interação implica que o efeito produzido pela variação de uma variável ( $x_{1}$, por exemplo) depende do nível da outra variável $\left(x_{2}\right)$. Por exemplo, os gráficos mostram que a variação de $x_{1}$ de 2 a 8 produz uma 
variação muito menor em $E(Y)$ quando $x_{2}=2$ do que quando $x_{2}=10$. Efeitos de interação ocorrem freqüentemente no estudo e na análise de sistemas reais, sendo os métodos de regressão uma das técnicas que se pode usar para descrevê-los.

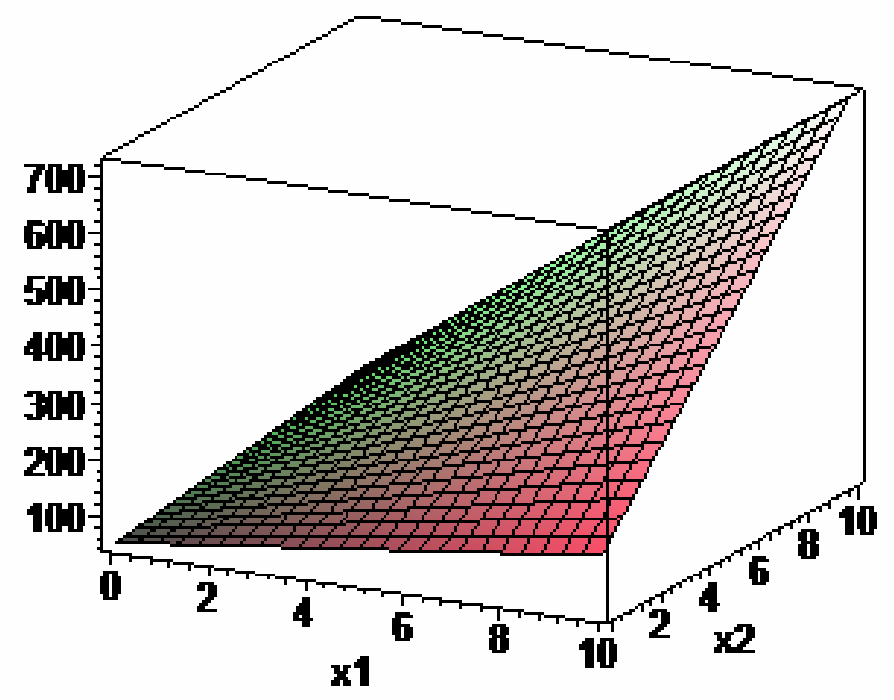

Figura 5.3 - Superfície de um modelo de regressão múltipla

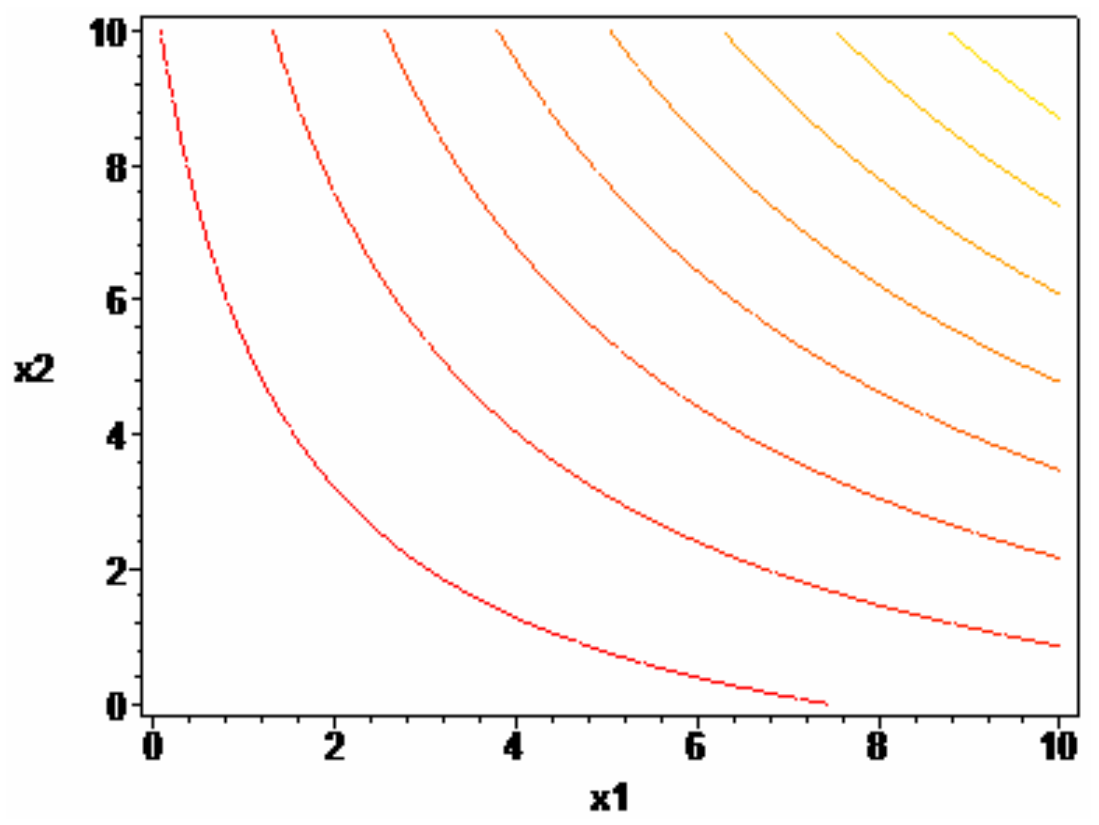

Figura 5.4 - Curvas de nível para um modelo de regressão múltipla

Como um exemplo final, tem-se o modelo de segunda ordem com interação 


$$
Y=\beta_{0}+\beta_{1} x_{1}+\beta_{2} x_{2}+\beta_{11} x_{1}^{2}+\beta_{22} x_{2}^{2}+\beta_{22} x_{2}^{2}+\beta_{12} x_{1} x_{2}+\epsilon
$$

Fazendo $x_{3}=x_{1}^{2}, x_{4}=x_{2}^{2}, x_{5}=x_{1} x_{2}, \beta_{3}=\beta_{11}, \beta_{4}=\beta_{22}$ e $\beta_{5}=\beta_{12}$, então a Equação (5.9) pode ser escrita como um modelo de regressão linear múltipla conforme segue:

$$
Y=\beta_{0}+\beta_{1} x_{1}+\beta_{2} x_{2}+\beta_{3} x_{3}+\beta_{4} x_{4}+\beta_{5} x_{5}+\epsilon
$$

As Figuras 5.5 e 5.6 mostram o gráfico tridimensional e a curva de nível. Estes gráficos indicam que a variação esperada por $\mathrm{Y}$ quando $\mathrm{x}_{1}$ for variado por uma unidade, por exemplo, é uma função de $\mathrm{x}_{1}$ e $\mathrm{x}_{2}$. Os termos quadráticos e de interação nesse modelo produzem uma função com forma de morro. Dependendo dos valores dos coeficientes de regressão, o modelo de segunda ordem com interação é capaz de considerar uma ampla variedade de formas; assim, ele é um modelo flexível de regressão.

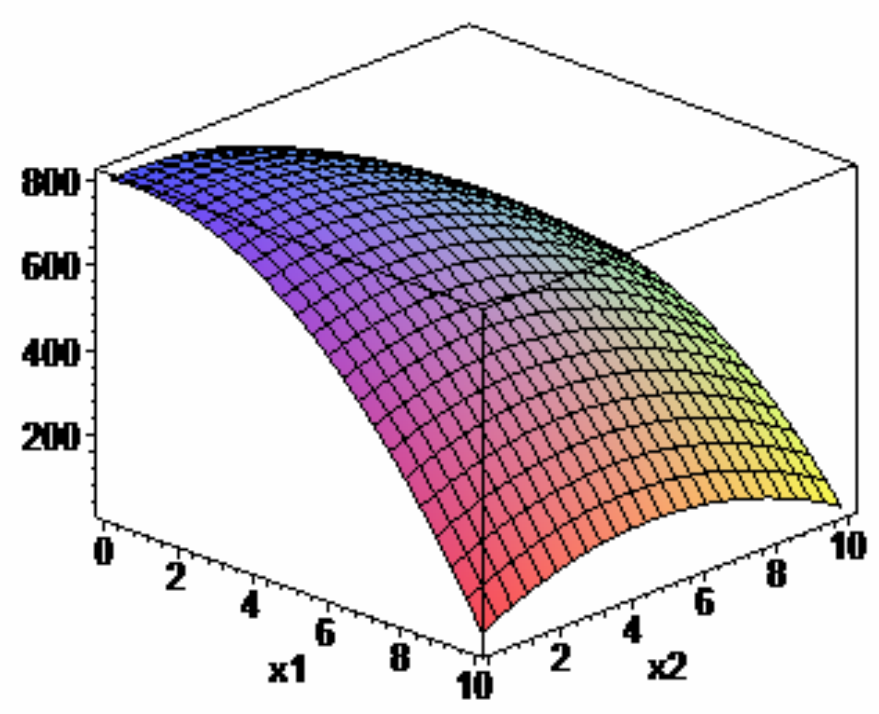

Figura 5.5 - Superfície de um modelo de regressão múltipla 


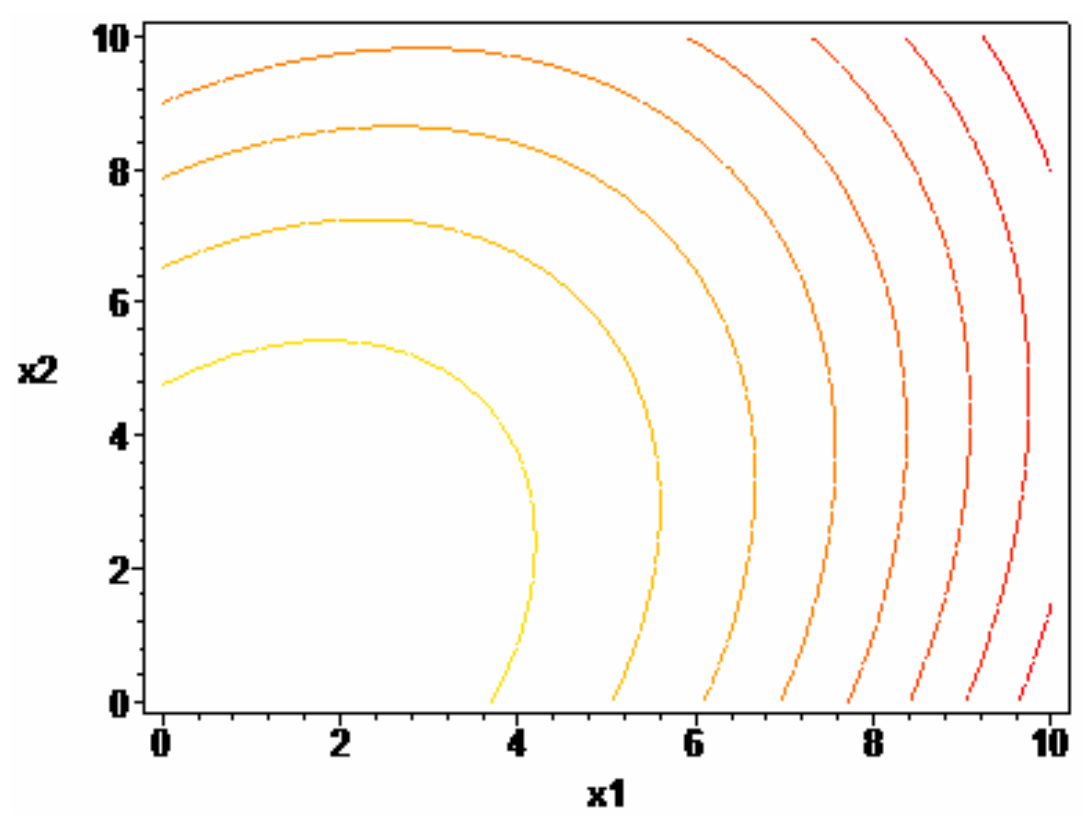

Figura 5.6 - Curvas de nível para um modelo de regressão múltipla

\subsubsection{Método dos Mínimos Quadrados}

O método dos mínimos quadrados pode ser usado para estimar os coeficientes de regressão no modelo de regressão múltipla, conforme a Equação (5.5). Supõe-se que $n>k$ observações estejam disponíveis e faz-se $x_{i j}$ denotar a i-ésima observação ou nível da variável $x_{j}$. As observações são:

$$
\left(x_{i 1}, x_{i 2}, \ldots, x_{i k}, y\right), \quad i=1,2, \ldots, n \quad \text { e } \quad n>k
$$

Cada observação $\left(x_{i 1}, x_{i 2}, \ldots, x_{i k}, y\right)$ satisfaz o modelo na Equação (5.5) ou

$$
y_{i}=\beta_{0}+\beta_{1} x_{i 1}+\beta_{2} x_{i 2}+\ldots+\beta_{k} x_{i k}+\epsilon_{i}=\beta_{0}+\sum_{j=1}^{k} \beta_{j} x_{i j}+\epsilon_{i}, \quad i=1,2, \ldots, n .
$$

A função dos mínimos quadrados é

$$
L=\sum_{i=1}^{n} \epsilon_{i}^{2}=\sum_{i=1}^{n}\left(y_{i}-\beta_{0}-\sum_{j=1}^{k} \beta_{j} x_{i j}\right)^{2}
$$


A função $L$ deve ser minimizada com relação a $\beta_{0}, \beta_{1}, \ldots, \beta_{k}$. As estimativas de mínimos quadrados de $\beta_{0}, \beta_{1}, \ldots, \beta_{\mathrm{k}}$ têm de satisfazer

$$
\left.\frac{\partial \mathrm{L}}{\partial \beta_{0}}\right|_{\hat{\beta}_{0}, \hat{\beta}_{1}, \ldots, \hat{\beta}_{k}}=-2 \sum_{\mathrm{i}=1}^{\mathrm{n}}\left(\mathrm{y}_{\mathrm{i}}-\hat{\beta}_{0}-\sum_{\mathrm{j}=1}^{\mathrm{k}} \hat{\beta}_{\mathrm{j}} \mathrm{x}_{\mathrm{ij}}\right)=0
$$

e

$$
\left.\frac{\partial L}{\partial \beta_{0}}\right|_{\hat{\beta}_{0}, \hat{\beta}_{1}, \ldots, \hat{\beta}_{k}}=-2 \sum_{i=1}^{n}\left(y_{i}-\hat{\beta}_{0}-\sum_{j=1}^{k} \hat{\beta}_{j} x_{i j}\right) x_{i j}=0, \quad j=1,2, \ldots, k
$$

Simplificando a Equação (5.13), obtemos as equações normais de mínimos quadrados:

$$
\begin{aligned}
& n \hat{\beta}_{0}+\hat{\beta}_{1} \sum_{i=1}^{n} x_{i 1}+\hat{\beta}_{2} \sum_{i=1}^{n} x_{i 2}+\ldots+\hat{\beta}_{k} \sum_{i=1}^{n} x_{i k}=\sum_{i=1}^{n} y_{i} \\
& \hat{\beta}_{0} \sum_{i=1}^{n} x_{i 1}+\hat{\beta}_{1} \sum_{i=1}^{n} x_{i 1}^{2}+\hat{\beta}_{2} \sum_{i=1}^{n} x_{i 1} x_{i 2}+\ldots+\hat{\beta}_{k} \sum_{i=1}^{n} x_{i 1} x_{i k}=\sum_{i=1}^{n} x_{i 1} y_{i} \\
& \vdots \\
& \hat{\beta}_{0} \sum_{i=1}^{n} x_{i k}+\hat{\beta}_{1} \sum_{i=1}^{n} x_{i k} x_{i 1}+\hat{\beta}_{2} \sum_{i=1}^{n} x_{i k} x_{i 2}+\ldots+\hat{\beta}_{k} \sum_{i=1}^{n} x_{i k}^{2}=\sum_{i=1}^{n} x_{i k} y_{i}
\end{aligned}
$$

Nota-se que há $p=k+1$ equações normais, uma para cada um dos coeficientes desconhecidos de regressão. A solução para as equações normais serão os estimadores de mínimos quadrados dos coeficientes de regressão, $\hat{\beta}_{0}, \hat{\beta}_{1}, \ldots, \hat{\beta}_{\mathrm{k}}$. As equações normais podem ser resolvidas por qualquer método apropriado para resolver um sistema de equações lineares. 


\subsubsection{Abordagem Matricial para a Regressão Linear Múltipla}

No ajuste de um modelo de regressão múltipla, é muito mais conveniente expressar as operações matemáticas usando a notação matricial. Supõe-se que existam $k$ regressores e $n$ observações $\left(x_{i 1}, x_{i 2}, \ldots, x_{i k}, y_{i}\right), i=1,2$, $\ldots, \mathrm{n}$, e que o modelo relacionando os regressores à resposta seja

$$
y_{i}=\beta_{0}+\beta_{1} x_{i 1}+\beta_{2} x_{i 2}+\ldots+\beta_{k} x_{i k}+\epsilon_{i}, \quad i=1,2, \ldots, n
$$

Esse modelo é um sistema de $n$ equações, que pode ser expresso na notação matricial como

$$
\underline{y}=\underline{X \beta}+\underline{\epsilon}
$$

sendo

$$
\underline{y}=\left[\begin{array}{c}
y_{1} \\
y_{2} \\
\vdots \\
y_{3}
\end{array}\right], \quad \underline{x}=\left[\begin{array}{ccccc}
1 & x_{11} & x_{12} & \cdots & x_{1 k} \\
1 & x_{21} & x_{22} & \cdots & x_{2 k} \\
\vdots & \vdots & \vdots & & \vdots \\
1 & x_{n 1} & x_{n 2} & \cdots & x_{n k}
\end{array}\right], \quad \underline{\beta}=\left[\begin{array}{c}
\beta_{0} \\
\beta_{1} \\
\vdots \\
\beta_{k}
\end{array}\right], \quad \underline{\epsilon}=\left[\begin{array}{c}
\epsilon_{1} \\
\epsilon_{2} \\
\vdots \\
\epsilon_{n}
\end{array}\right]
$$

Em geral, y é um vetor $(n \times 1)$ das observações, $\underline{X}$ é uma matriz $(n \times p)$ dos níveis das variáveis independentes, $\beta$ é um vetor $(n \times 1)$ dos erros aleatórios.

Deseja-se encontrar o vetor dos estimadores de mínimos quadrados, $\underline{\hat{\beta}}$, que minimiza

$$
L=\sum_{i=1}^{n} \epsilon_{i}^{2}=\underline{\epsilon}^{\prime} \in=(\underline{y}-\underline{X \beta})^{\prime}(\underline{y}-\underline{X \beta})
$$

O estimador de mínimos quadrados $\underline{\beta}$ é a solução para $\underline{\beta}$ nas equações 


$$
\frac{\partial \mathrm{L}}{\partial \underline{\beta}}=\underline{0} .
$$

As equações resultantes que têm de ser resolvidas são:

$$
\underline{X} \underline{X}=\underline{X^{\prime} y} .
$$

A Equação (5.16) são as equações normais de mínimos quadrados na forma matricial idênticas à forma escalar das equações normais dadas, anteriormente, pela Equação (5.14). Com o objetivo de resolver as equações normais, multiplicando-se ambos os lados da Equação (5.16) pelo inverso de $\underline{X^{\prime}} \underline{X}$. Conseqüentemente, a estimativa de mínimos quadrados de $\underline{\beta}$ é

$$
\underline{\hat{\beta}}=\left(\underline{X^{\prime} X}\right)^{-1} \underline{X^{\prime} y} .
$$

Observa-se que há $\mathrm{p}=\mathrm{k}+1$ equações normais para $\mathrm{p}=\mathrm{k}+1$ incógnitas (os valores de $\hat{\beta}_{0}, \hat{\beta}_{1}, \ldots, \hat{\beta}_{\mathrm{k}}$ ). Além disso, a matriz $\underline{X}^{\prime} \underline{X}$ é freqüentemente não singular, como foi considerado anteriormente, de modo que os métodos descritos nos livros textos sobre determinantes e matrizes para inverter essas matrizes podem ser usados para encontrar $\left(\underline{X^{\prime}} \underline{X}\right)^{-1}$. Na prática, os cálculos de regressão múltipla são quase sempre realizados em um computador.

É fácil ver que a forma matricial das equações normais é idêntica à forma escalar. Escrevendo a Equação (5.16) em detalhes, obtém-se:

$$
\left[\begin{array}{ccccc}
n & \sum_{i=1}^{n} x_{i 1} & \sum_{i=1}^{n} x_{i 2} & \cdots & \sum_{i=1}^{n} x_{i k} \\
\sum_{i=1}^{n} x_{i 1} & \sum_{i=1}^{n} x_{i 1}^{2} & \sum_{i=1}^{n} x_{i 1} x_{i 2} & \cdots & \sum_{i=1}^{n} x_{i 1} x_{i k} \\
\vdots & \vdots & \vdots & & \vdots \\
\sum_{i=1}^{n} x_{i k} & \sum_{i=1}^{n} x_{i k} x_{i 1} & \sum_{i=1}^{n} x_{i 1} x_{i 2} & \cdots & \sum_{i=1}^{n} x_{i k}^{2}
\end{array}\right]\left[\begin{array}{c}
\beta_{0} \\
\hat{\beta}_{1} \\
\vdots \\
\hat{\beta}_{k}
\end{array}\right]=\left[\begin{array}{c}
\sum_{i=1}^{n} y_{i} \\
\sum_{i=1}^{n} x_{i 1} y_{i} \\
\vdots \\
\sum_{i=1}^{n} x_{i k} y_{i}
\end{array}\right] .
$$


Se a multiplicação matricial indicada for feita, resultará a forma escalar das equações normais. Nessa forma, é fácil ver que $\underline{X^{\prime}} \underline{X}$ é uma matriz simétrica $(p \times p)$ e $\underline{X} \underline{y}$ é um vetor coluna $(p \times 1)$. Pode-se notar a estrutura especial da matriz $\underline{X^{\prime}} \underline{X}$. Os elementos da diagonal de $\underline{X^{\prime}} \underline{X}$ são as somas dos quadrados dos elementos das colunas de $\underline{X}$, e os elementos fora da diagonal são as somas dos produtos cruzados dos elementos nas colunas de $\underline{X}$. Além disso, nota-se que os elementos de $\underline{X} \underline{y}$ são as somas dos produtos cruzados das colunas de $\underline{X}$ e das observações $\left\{y_{i}\right\}$.

O modelo ajustado de regressão é

$$
\hat{y}_{i}=\hat{\beta}_{0}+\sum_{j=1}^{k} \hat{\beta}_{j} x_{i j}, \quad i=1,2, \ldots, n .
$$

Na notação matricial, o modelo ajustado é

$$
\underline{\hat{y}}=\underline{X \hat{\beta}} .
$$

A diferença entre a observação $y_{i}$ e o valor ajustado $\hat{y}_{i}$ é um resíduo, $e_{i}=y_{i}-\hat{y}_{i} . O$ vetor $(n \times 1)$ dos resíduos é denotado por

$$
\underline{\mathrm{e}}=\underline{\mathrm{y}}-\underline{\hat{y}}
$$

\subsection{Desenvolvimento da Superfície de Resposta}

Uma vez definida a forma da SR, propõe-se com o MSR desenvolvê-la em torno do ponto de ruptura mais provável $\left(P^{*}\right)$, que tem posição desconhecida. A solução proposta consiste em procurar uma seqüência de pontos $P^{*}(k)$ associada a uma seqüência de $S R(k)$ na qual o domínio de definição contém $\mathrm{P}^{*}(\mathrm{k})$. Este procedimento não garante uma convergência para 
um mínimo global, portanto, a existência de mínimos locais pode induzir a contribuições significativas na probabilidade de ruptura. Em todos os casos, deve ser feita uma análise dos resultados para validá-los.

A construção de uma SR de acordo com o MSR pode ser dividida em etapas, que serão descritas a seguir:

\subsubsection{Definição do Espaço de Trabalho}

Há duas possibilidades para construir uma SR: no espaço físico e no espaço reduzido ou normal padrão. O espaço físico apresenta a vantagem de se obter diretamente uma solução física para o problema, de acordo com a experiência do projetista. Entretanto, a escolha de pontos físicos pode conduzir a pontos padronizados situados muito longe da origem no espaço normal padrão $(U)$ se o desvio padrão for inapto ou os pontos forem próximos das margens. Além disso, o projetista conhece bem a média da área processada (área sobre os pontos), mas pode ter dificuldades para realizações em torno da ruptura a ser identificada. Finalmente, a qualidade da solução de confiabilidade depende do desenvolvimento da função de estado limite obtido por variáveis normalizadas.

Um estudo a priori de domínios admissíveis das variáveis físicas induz, através de transformações isoprobabilísticas, ao domínio padronizado no qual é válido o ponto de projeto. De acordo com as características citadas acima, conclui-se que é mais razoável para o estudo de confiabilidade trabalhar no espaço normal padrão.

\subsubsection{Transformações de Distribuições de Probabilidade}

É mais conveniente trabalhar no espaço normal padrão, como já discutido, só que as variáveis de projeto são fornecidas e interpretadas no espaço físico. Portanto, necessitam-se transformações isoprobabilísticas no 
desenvolvimento do modelo de confiabilidade utilizado. É necessário um largo uso de transformações, ou mudanças de espaços de trabalho, em cada loopping de busca do índice de confiabilidade. Portanto, é essencial para a análise mecânica-probabilística a construção de uma transformação $T$, que passa as variáveis do espaço físico, representado por x, para o normal padrão, representado por $u$, e vice-versa.

\subsubsection{Variáveis Aleatórias Independentes}

Nos casos onde as variáveis aleatórias de base são independentes, a transformação é feita para cada variável independentemente, apenas igualando as funções de repartição:

$$
\phi\left(u_{i}\right)=F_{x_{i}}\left(x_{i}\right)
$$

A transformação direta é então:

$$
\mathrm{T}(\mathrm{x}) \Rightarrow \mathrm{u}_{\mathrm{i}}=\phi^{-1}\left(\mathrm{~F}_{\mathrm{x}_{\mathrm{i}}}\left(\mathrm{x}_{\mathrm{i}}\right)\right) \quad \mathrm{i}=1, \ldots, \mathrm{n}
$$

A transformação inversa é:

$$
\mathrm{T}^{-1}(\mathrm{U}) \Rightarrow \mathrm{x}_{\mathrm{i}}=\mathrm{F}_{\mathrm{x}_{\mathrm{i}}}^{-1}\left(\phi\left(\mathrm{u}_{\mathrm{i}}\right)\right) \quad \mathrm{i}=1, \ldots, \mathrm{n}
$$

Em notação matricial:

$$
\begin{aligned}
& \mathrm{U}=\{\mathrm{u}\}=\left\{\phi^{-1}\left(\mathrm{~F}_{\{\mathbf{x}\}}(\{\mathbf{x}\})\right)\right\} \\
& \mathrm{X}=\{\mathbf{x}\}=\left\{\mathrm{F}_{\{\mathbf{x}\}}^{-1}(\phi(\{\mathbf{x}\}))\right\}
\end{aligned}
$$

onde $x_{i}$ e $u_{i}$ representam as variáveis aleatórias no espaço físico e reduzido, respectivamente.

Na prática, o índice de confiabilidade $(\beta)$ é calculado no espaço normal padrão, ou seja, a variável $u_{i}$ é representada por uma distribuição normal com média nula e desvio padrão unitário. Desta forma, a função que representa ui 
não é integrável analiticamente, sendo necessária uma integração numérica da função. Baseado em testes é aconselhável utilizar um procedimento Gaussiano, com a região de integração dividida em sub-regiões. Para integrar cada sub-região deve-se utilizar dez pontos de Gauss. As funções de distribuição acumuladas adequadas às variáveis de engenharia, mesmo no espaço físico, em geral não são possíveis de se integrar analiticamente, sendo necessário o uso de integração numérica.

\subsubsection{Distribuição Normal Equivalente}

O método consiste em fazer a transformação das variáveis uma a uma, apresentando solução exata apenas nos casos em que as variáveis aleatórias são independentes. Após encontrar os parâmetros da distribuição normal equivalente à distribuição original, facilmente são feitas transformações isoprobabilística do espaço normal equivalente para o espaço normal padrão e vice-versa. Pode-se dizer que esta é a forma mais simples de se transformar as variáveis para o espaço reduzido.

Para encontrar os parâmetros da distribuição normal equivalente $\mu_{\mathrm{i}}$ e $\sigma_{\mathrm{i}}$, para a variável $x_{i}$, com uma função de densidade $f_{x i}\left(x_{i}\right)$ e uma função de distribuição acumulada $F_{x_{i}}\left(x_{i}\right)$, deve ser feito o procedimento descrito a seguir. $\Phi$ e $\varphi$ representam, respectivamente, as funções de distribuição acumulada e de densidade normal equivalente.

Igualando as funções de distribuição acumuladas no ponto considerado:

$$
\phi\left(\frac{x_{i}-\mu_{i}}{\sigma_{i}}\right)=F_{x_{i}}\left(x_{i}\right)
$$

Igualar as funções de densidade no mesmo ponto, que é obtida derivando a Equação(5.24) em relação a $x_{i}$ :

$$
\frac{1}{\sigma_{i}} \varphi\left(\frac{x_{i}-\mu_{i}}{\sigma_{i}}\right)=f_{x_{i}}\left(x_{i}\right)
$$


De (5.24) tem-se:

$$
\frac{x_{i}-\mu_{i}}{\sigma_{i}}=\phi^{-1}\left[F_{x_{i}}\left(x_{i)}\right]\right.
$$

Substituindo-se a relação (5.26) na (5.25), pode-se determinar uma expressão para o desvio padrão da distribuição normal equivalente:

$$
\sigma_{i}=\frac{\varphi\left(\phi^{-1}\left[F_{x_{i}}\left(x_{i}\right)\right]\right.}{f_{x_{i}}\left(x_{i}\right)}
$$

Reorganizando-se (5.24), tem-se a média da distribuição normal equivalente:

$$
\mu_{i}=x_{i}-\sigma_{i} \phi^{-1}\left[F_{x_{i}}\left(x_{i}\right)\right]
$$

\subsection{Planos de Experiência}

Os planos de experiência podem ser classificados em duas grandes categorias: o plano de experiência numérico e o plano de experiência aleatório. A diferença entre um PE numérico e um PE aleatório é que para duas experiências com dados idênticos, ao utilizar um PE numérico a igualdade entre os resultados é total, o que não acontece com planos de experiência aleatórios.

Os planos de experiência aleatórios podem conduzir a singularidades no sistema, exigindo um número maior de pontos para evitar essa singularidade. Uma outra característica é que os pontos não se localizam necessariamente em torno da solução devido à aleatoriedade, podendo necessitar de mais superfícies de resposta para a convergência do modelo. Entretanto, para problemas com elevado número de variáveis aleatórias pode se tornar difícil estabelecer um PE numérico que garanta convergência. Nessas situações o PE aleatório pode ser mais indicado. 
Plano de experiência numérico é um conjunto de valores determinísticos com os quais será aproximada a hipersuperfície da resposta estrutural. Ou seja, supõe-se um sistema de coordenadas, onde cada uma delas equivale a uma variável aleatória de resistência e uma outra equivale à resposta estrutural. O PE é responsável pela variação determinística das variáveis aleatórias para gerar a hipersuperfície da resposta estrutural também determinística, a qual definirá a hipersuperfície de ruptura do problema mecânico-probabilístico.

Existem várias formas de PE disponíveis em bibliografias. Entretanto, necessita-se definir os coeficientes que se encaixam melhor ao problema estudado. Estes PE se classificam como planos de experiência numéricos clássicos e podem ser vistos na Figura 5.7. É importante observar que os PE são construídos no espaço reduzido.

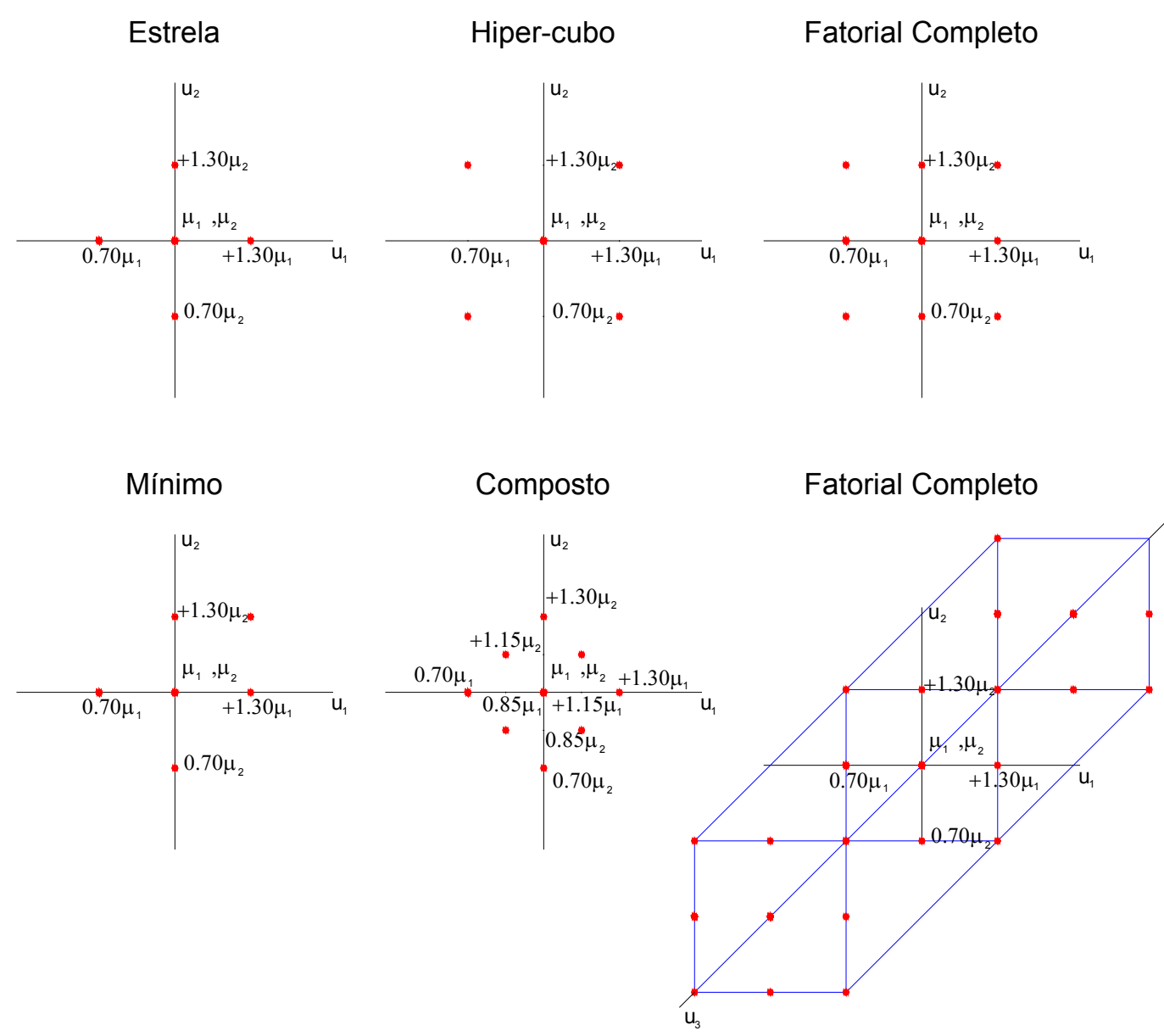

Figura 5.7 - Planos de experiência numéricos adotados no presente trabalho 
Os planos de experiência para um número de variáveis aleatórias superior a duas são semelhantes aos listados na Figura 5.7, acrescentados de pontos posicionados como os descritos nos dois primeiros eixos, $u_{1}$ e $u_{2}$. $O$ número de novos pontos depende do número de coeficientes do polinômio aproximador $\left(\mathrm{n}_{\mathrm{c}}\right)$ da superfície de resposta.

Na Tabela 5.1 está listado o número de pontos $\left(n_{p}\right)$ de cada plano, que é função do número de coeficientes $\mathrm{n}_{\mathrm{c}}$ do polinômio aproximador da $\mathrm{SR}$ e do número de variáveis aleatórias $\left(\mathrm{n}_{\mathrm{va}}\right)$. Para um polinômio aproximador do segundo grau, tem-se a seguinte relação entre o número de v.a. e o número de coeficientes:

\begin{tabular}{|c|l|l|l|l|l|l|}
\hline v.a. $=\mathbf{n}_{\mathrm{VA}}$ & 1 & 2 & 3 & 4 & 5 & 6 \\
\hline $\mathbf{n}_{\mathbf{c}}$ & 3 & 6 & 10 & 15 & 21 & 28 \\
\hline
\end{tabular}

Tabela 5.1 - Número de coeficientes em relação ao número de v.a

- Plano estrela: $\left\{\begin{array}{c}\mathrm{n}_{\mathrm{VA}}=1 \therefore \mathrm{n}_{\mathrm{p}}=\mathrm{n}_{\mathrm{c}} \\ \mathrm{n}_{\mathrm{VA}}>1 \therefore \mathrm{n}_{\mathrm{p}}=\mathrm{n}_{\mathrm{c}}-1\end{array}\right.$

- Plano estrela 45 ou hiper-cubo: $\left\{\begin{array}{c}\mathrm{n}_{\mathrm{VA}}=1 \therefore \mathrm{n}_{\mathrm{p}}=\mathrm{n}_{\mathrm{c}} \\ \mathrm{n}_{\mathrm{VA}}>1 \therefore \mathrm{n}_{\mathrm{p}}=\mathrm{n}_{\mathrm{c}}-1\end{array}\right.$

- Plano fatorial completo: $\left\{n_{p}=3^{n_{V A}}\right.$

- Plano mínimo: $\left\{\mathrm{n}_{\mathrm{p}}=\mathrm{n}_{\mathrm{c}}\right.$

- Plano composto: $\left\{\begin{array}{c}\mathrm{n}_{\mathrm{VA}}=1 \therefore \mathrm{n}_{\mathrm{p}}=5 \\ \mathrm{n}_{\mathrm{VA}}>1 \therefore \mathrm{n}_{\mathrm{p}}=9\left(\mathrm{n}_{\mathrm{VA}}-1\right)\end{array}\right.$

Os planos de experiência são responsáveis pelas superfícies de resposta adaptativas requeridas pelo MSR desde o início do processo iterativo 
até a convergência. A velocidade de convergência, ou custo computacional do método depende de uma boa escolha desses planos.

Na construção de um PE é interessante ponderar seus coeficientes com características implícitas das variáveis aleatórias. No caso, optou-se por construir os planos de experiência ponderando seus coeficientes com os momentos estatísticos das variáveis aleatórias envolvidas. Para isso, considere a variável definida na Equação (5.29).

$$
\delta_{\mathrm{i}}=\frac{\sigma_{\mathrm{i}}}{\overline{\mathrm{x}_{\mathrm{i}}}}
$$

onde $\bar{x}_{i}$ e $\sigma_{1}$ referem-se a média e o desvio padrão da variável i, respectivamente.

\subsubsection{Plano de Experiência Numérico Estrela}

Uma observação a ser feita para os planos de experiência Estrela e Hiper-cubo é com relação aos seus números de pontos. Nota-se que o número de pontos é menor que o número de coeficientes do polinômio de segundo grau utilizado para aproximar a RS da estrutura. Portanto, para encontrar os coeficientes do polinômio através de regressão alguns de seus termos devem ser eliminados para evitar a singularidade do sistema de equações. Preferencialmente não deve ser eliminado o termo independente nem os termos que dependem exclusivamente de uma única variável aleatória. Como não se conhecem as respostas do problema a eliminação desses termos pode empobrecer o algoritmo.

Os coeficientes propostos para problemas com 1 variável aleatória são:

$$
\left\{\begin{array}{lll}
\overline{\mathrm{x}}_{1} & \overline{\mathrm{x}}_{1}\left(1-3 \delta_{1}\right) & \overline{\mathrm{x}}_{1}\left(1+3 \delta_{1}\right.
\end{array}\right\}
$$

Os coeficientes propostos para problemas com 2 variáveis aleatórias são: 


$$
\left[\begin{array}{cc}
\overline{\mathrm{x}}_{1} & \overline{\mathrm{x}}_{2} \\
\overline{\mathrm{x}}_{1}\left(1-3 \delta_{1}\right) & \overline{\mathrm{x}}_{2} \\
\overline{\mathrm{x}}_{1}\left(1+3 \delta_{1}\right) & \overline{\mathrm{x}}_{2} \\
\overline{\mathrm{x}}_{1} & \overline{\mathrm{x}}_{2}\left(1-3 \delta_{2}\right) \\
\overline{\mathrm{x}}_{1} & \overline{\mathrm{x}}_{2}\left(1+3 \delta_{2}\right)
\end{array}\right]
$$

Os coeficientes propostos para problemas com 3 variáveis aleatórias são:

$$
\left[\begin{array}{ccc}
\overline{\mathrm{x}}_{1} & \overline{\mathrm{x}}_{2} & \overline{\mathrm{x}}_{3} \\
\overline{\mathrm{x}}_{1}\left(1-3 \delta_{1}\right) & \overline{\mathrm{x}}_{2} & \overline{\mathrm{x}}_{3} \\
\overline{\mathrm{x}}_{1}\left(1+3 \delta_{1}\right) & \overline{\mathrm{x}}_{2} & \overline{\mathrm{x}}_{3} \\
\overline{\mathrm{x}}_{1} & \overline{\mathrm{x}}_{2}\left(1-3 \delta_{2}\right) & \overline{\mathrm{x}}_{3} \\
\overline{\mathrm{x}}_{1} & \overline{\mathrm{x}}_{2}\left(1+3 \delta_{2}\right) & \overline{\mathrm{x}}_{3} \\
\overline{\mathrm{x}}_{1} & \overline{\mathrm{x}}_{2} & \overline{\mathrm{x}}_{3}\left(1-3 \delta_{3}\right) \\
\overline{\mathrm{x}}_{1}\left(1-3 \delta_{1}\right) & \overline{\mathrm{x}}_{2} & \overline{\mathrm{x}}_{3}\left(1-3 \delta_{3}\right) \\
\overline{\mathrm{x}}_{1}\left(1+3 \delta_{1}\right) & \overline{\mathrm{x}}_{2} & \overline{\mathrm{x}}_{3}\left(1-3 \delta_{3}\right) \\
\overline{\mathrm{x}}_{1} & \overline{\mathrm{x}}_{2}\left(1-3 \delta_{2}\right) & \overline{\mathrm{x}}_{3}\left(1-3 \delta_{3}\right)
\end{array}\right]
$$

Os coeficientes propostos para problemas com 4 variáveis aleatórias são:

$$
\left[\begin{array}{cccc}
\overline{\mathrm{x}}_{1} & \overline{\mathrm{x}}_{2} & \overline{\mathrm{x}}_{3} & \overline{\mathrm{x}}_{4} \\
\overline{\mathrm{x}}_{1}\left(1-3 \delta_{1}\right) & \overline{\mathrm{x}}_{2} & \overline{\mathrm{x}}_{3}\left(1-3 \delta_{3}\right) & \overline{\mathrm{x}}_{4}\left(1-3 \delta_{4}\right) \\
\overline{\mathrm{x}}_{1}\left(1+3 \delta_{1}\right) & \overline{\mathrm{x}}_{2} & \overline{\mathrm{x}}_{3}\left(1-3 \delta_{3}\right) & \overline{\mathrm{x}}_{4}\left(1-3 \delta_{4}\right) \\
\overline{\mathrm{x}}_{1} & \overline{\mathrm{x}}_{2}\left(1-3 \delta_{2}\right) & \overline{\mathrm{x}}_{3}\left(1-3 \delta_{3}\right) & \overline{\mathrm{x}}_{4}\left(1-3 \delta_{4}\right) \\
\overline{\mathrm{x}}_{1} & \overline{\mathrm{x}}_{2}\left(1+3 \delta_{2}\right) & \overline{\mathrm{x}}_{3}\left(1-3 \delta_{3}\right) & \overline{\mathrm{x}}_{4}\left(1-3 \delta_{4}\right) \\
\overline{\mathrm{x}}_{1} & \overline{\mathrm{x}}_{2} & \overline{\mathrm{x}}_{3}\left(1+3 \delta_{3}\right) & \overline{\mathrm{x}}_{4}\left(1+3 \delta_{4}\right) \\
\overline{\mathrm{x}}_{1}\left(1-3 \delta_{1}\right) & \overline{\mathrm{x}}_{2} & \overline{\mathrm{x}}_{3}\left(1+3 \delta_{3}\right) & \overline{\mathrm{x}}_{4}\left(1+3 \delta_{4}\right) \\
\overline{\mathrm{x}}_{1}\left(1+3 \delta_{1}\right) & \overline{\mathrm{x}}_{2} & \overline{\mathrm{x}}_{3}\left(1+3 \delta_{3}\right) & \overline{\mathrm{x}}_{4}\left(1+3 \delta_{4}\right) \\
\overline{\mathrm{x}}_{1} & \overline{\mathrm{x}}_{2}\left(1-3 \delta_{2}\right) & \overline{\mathrm{x}}_{3}\left(1+3 \delta_{3}\right) & \overline{\mathrm{x}}_{4}\left(1+3 \delta_{4}\right) \\
\overline{\mathrm{x}}_{1} & \overline{\mathrm{x}}_{2}\left(1+3 \delta_{2}\right) & \overline{\mathrm{x}}_{3}\left(1+3 \delta_{3}\right) & \overline{\mathrm{x}}_{4}\left(1+3 \delta_{4}\right) \\
\overline{\mathrm{x}}_{1} & \overline{\mathrm{x}}_{2} & \overline{\mathrm{x}}_{3}\left(1-1.5 \delta_{3}\right) & \overline{\mathrm{x}}_{4}\left(1-1.5 \delta_{4}\right) \\
\overline{\mathrm{x}}_{1}\left(1-3 \delta_{1}\right) & \overline{\mathrm{x}}_{2} & \overline{\mathrm{x}}_{3}\left(1-1.5 \delta_{3}\right) & \overline{\mathrm{x}}_{4}\left(1-1.5 \delta_{4}\right) \\
\overline{\mathrm{x}}_{1}\left(1+3 \delta_{1}\right) & \overline{\mathrm{x}}_{2} & \overline{\mathrm{x}}_{3}\left(1-1.5 \delta_{3}\right) & \overline{\mathrm{x}}_{4}\left(1-1.5 \delta_{4}\right) \\
\overline{\mathrm{x}}_{1} & \overline{\mathrm{x}}_{2}\left(1-3 \delta_{2}\right) & \overline{\mathrm{x}}_{3}\left(1-1.5 \delta_{3}\right) & \overline{\mathrm{x}}_{4}\left(1-1.5 \delta_{4}\right)
\end{array}\right]
$$


Os coeficientes propostos para problemas com 5 variáveis aleatórias são:

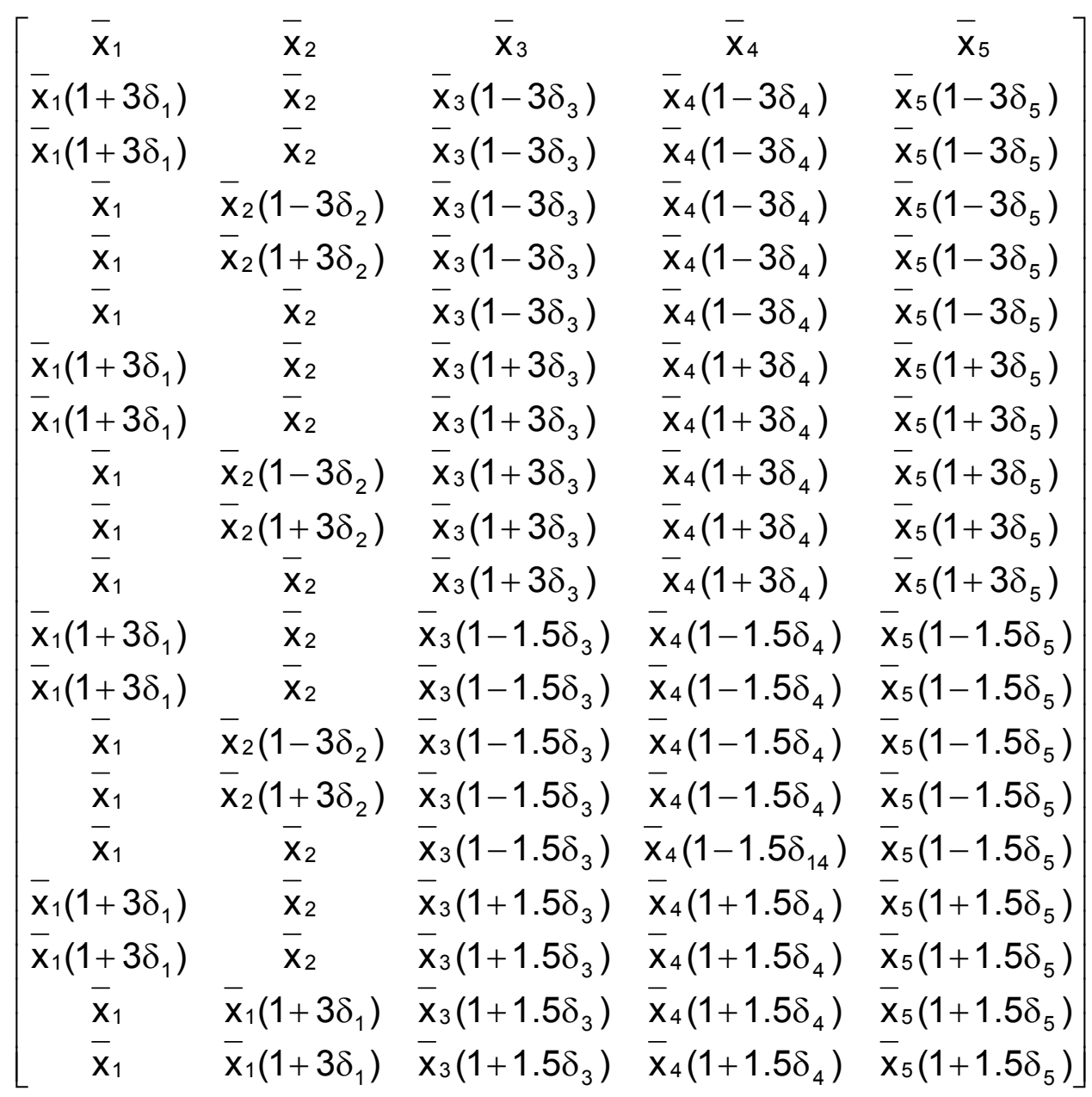

Os coeficientes propostos para problemas com 6 variáveis aleatórias são: 


\begin{tabular}{|c|c|c|c|c|c|}
\hline- & - & - & - & - & - \\
\hline$-\mathrm{X}_{1}$ & $\mathrm{X}_{2}$ & $\mathrm{X}_{3}$ & $\mathrm{X}_{4}$ & $\mathrm{X}_{5}$ & $\mathrm{X}_{6}$ \\
\hline $\mathrm{x}_{1}\left(1-3 \delta_{1}\right)$ & $\bar{x}_{2}$ & $\bar{x}_{3}\left(1-3 \delta_{3}\right)$ & $\mathrm{x}_{4}\left(1-3 \delta_{4}\right)$ & $\bar{x}_{5}\left(1-3 \delta_{5}\right)$ & $\mathrm{x}_{6}\left(1-3 \delta_{6}\right)$ \\
\hline $\mathrm{x}_{1}\left(1+3 \delta_{1}\right)$ & $\mathrm{x}_{2}$ & $\mathrm{x}_{3}\left(1-3 \delta_{3}\right)$ & $\mathrm{x}_{4}\left(1-3 \delta_{4}\right)$ & $\mathrm{x}_{5}\left(1-3 \delta_{5}\right)$ & $\mathrm{x}_{6}\left(1-3 \delta_{6}\right)$ \\
\hline$\overline{\mathrm{x}}_{1}$ & $\overline{\mathrm{x}}_{2}\left(1-3 \delta_{2}\right)$ & $\overline{\mathrm{x}}_{3}\left(1-3 \delta_{3}\right)$ & $\overline{\mathrm{x}}_{4}\left(1-3 \delta_{4}\right)$ & $\overline{\mathrm{X}}_{5}\left(1-3 \delta_{5}\right)$ & $\overline{\mathrm{x}}_{6}\left(1-3 \delta_{6}\right)$ \\
\hline$\overline{\mathrm{X}}_{1}$ & $\overline{\mathrm{x}}_{2}\left(1+3 \delta_{2}\right)$ & $\overline{\mathrm{x}}_{3}\left(1-3 \delta_{3}\right)$ & $\overline{\mathrm{x}}_{4}\left(1-3 \delta_{4}\right)$ & $\overline{\mathrm{X}}_{5}\left(1-3 \delta_{5}\right)$ & $\overline{\mathrm{x}}_{6}\left(1-3 \delta_{6}\right)$ \\
\hline$\overline{\mathrm{x}}_{1}$ & $\overline{\mathrm{x}}_{2}$ & $\overline{\mathrm{x}}_{3}\left(1-3 \delta_{3}\right)$ & $\overline{\mathrm{x}}_{4}\left(1-3 \delta_{4}\right)$ & $\overline{\mathrm{X}}_{5}\left(1-3 \delta_{5}\right)$ & $\overline{\mathrm{x}}_{6}\left(1-3 \delta_{6}\right)$ \\
\hline$\overline{\mathrm{x}}_{1}\left(1-3 \delta_{1}\right)$ & $\overline{\mathrm{X}}_{2}$ & $\overline{\mathrm{x}}_{3}\left(1+3 \delta_{3}\right)$ & $\overline{\mathrm{x}}_{4}\left(1+3 \delta_{4}\right)$ & $\overline{\mathrm{x}}_{5}\left(1+3 \delta_{5}\right)$ & $\bar{x}_{6}\left(1+3 \delta_{6}\right)$ \\
\hline$\overline{\mathrm{x}}_{1}\left(1+3 \delta_{1}\right)$ & $\overline{\mathrm{x}}_{2}$ & $\overline{\mathrm{x}}_{3}\left(1+3 \delta_{3}\right)$ & $\overline{\mathrm{x}}_{4}\left(1+3 \delta_{4}\right)$ & $\overline{\mathrm{X}}_{5}\left(1+3 \delta_{5}\right)$ & $\overline{\mathrm{x}}_{6}\left(1+3 \delta_{6}\right)$ \\
\hline$\overline{\mathrm{X}}_{1}$ & $\overline{\mathrm{x}}_{2}\left(1-3 \delta_{2}\right)$ & $\bar{x}_{3}\left(1+3 \delta_{3}\right)$ & $\overline{\mathrm{x}}_{4}\left(1+3 \delta_{4}\right)$ & $\overline{\mathrm{x}}_{5}\left(1+3 \delta_{5}\right)$ & $\bar{x}_{6}\left(1+3 \delta_{6}\right)$ \\
\hline$\overline{\mathrm{X}}_{1}$ & $\bar{x}_{2}\left(1+3 \delta_{2}\right)$ & $\overline{\mathrm{x}}_{3}\left(1+3 \delta_{3}\right)$ & $\overline{\mathrm{x}}_{4}\left(1+3 \delta_{4}\right)$ & $\overline{\mathrm{X}}_{5}\left(1+3 \delta_{5}\right)$ & $\bar{x}_{6}\left(1+3 \delta_{6}\right)$ \\
\hline$\overline{\mathrm{X}}_{1}$ & $\overline{\mathrm{X}}_{2}$ & $\bar{x}_{3}\left(1+3 \delta_{3}\right)$ & $\overline{\mathrm{x}}_{4}\left(1+3 \delta_{4}\right)$ & $\overline{\mathrm{X}}_{5}\left(1+3 \delta_{5}\right)$ & $\overline{\mathrm{x}}_{6}\left(1+3 \delta_{6}\right)$ \\
\hline $\bar{x}_{1}\left(1-3 \delta_{1}\right)$ & $\overline{\mathrm{X}}_{2}$ & $\overline{\mathrm{x}}_{3}\left(1-1.5 \delta_{3}\right)$ & $\overline{\mathrm{X}}_{4}\left(1-1.5 \delta_{4}\right)$ & $\overline{\mathrm{X}}_{5}\left(1-1.5 \delta_{5}\right)$ & $\bar{x}_{6}\left(1-1.5 \delta_{6}\right)$ \\
\hline$\overline{\mathrm{x}}_{1}\left(1+3 \delta_{1}\right)$ & $\overline{\mathrm{x}}_{2}$ & $\overline{\mathrm{x}}_{3}\left(1-1.5 \delta_{3}\right)$ & $\overline{\mathrm{X}}_{4}\left(1-1.5 \delta_{4}\right)$ & $\overline{\mathrm{X}}_{5}\left(1-1.5 \delta_{5}\right)$ & $\overline{\mathrm{x}}_{6}\left(1-1.5 \delta_{6}\right)$ \\
\hline$\overline{\mathrm{x}}_{1}$ & $\bar{x}_{2}\left(1-3 \delta_{2}\right)$ & $\overline{\mathrm{x}}_{3}\left(1-1.5 \delta_{3}\right)$ & $\overline{\mathrm{X}}_{4}\left(1-1.5 \delta_{4}\right)$ & $\overline{\mathrm{X}}_{5}\left(1-1.5 \delta_{5}\right)$ & $\overline{\mathrm{x}}_{6}\left(1-1.5 \delta_{6}\right)$ \\
\hline$\overline{\mathrm{X}}_{1}$ & $\overline{\mathrm{x}}_{2}\left(1+3 \delta_{2}\right)$ & $\overline{\mathrm{x}}_{3}\left(1-1.5 \delta_{3}\right)$ & $\overline{\mathrm{x}}_{4}\left(1-1.5 \delta_{4}\right)$ & $\overline{\mathrm{X}}_{5}\left(1-1.5 \delta_{5}\right)$ & $\overline{\mathrm{X}}_{6}\left(1-1.5 \delta_{6}\right)$ \\
\hline$\overline{\mathrm{X}}_{1}$ & $\overline{\mathrm{X}}_{2}$ & $\overline{\mathrm{x}}_{3}\left(1-1.5 \delta_{3}\right)$ & $\overline{\mathrm{X}}_{4}\left(1-1.5 \delta_{4}\right)$ & $\overline{\mathrm{X}}_{5}\left(1-1.5 \delta_{5}\right)$ & $\overline{\mathrm{x}}_{6}\left(1-1.5 \delta_{6}\right)$ \\
\hline$\overline{\mathrm{x}}_{1}\left(1-3 \delta_{1}\right)$ & $\overline{\mathrm{x}}_{2}$ & $\overline{\mathrm{x}}_{3}\left(1+1.5 \delta_{3}\right)$ & $\overline{\mathrm{X}}_{4}\left(1+1.5 \delta_{4}\right)$ & $\overline{\mathrm{x}}_{5}\left(1+1.5 \delta_{5}\right)$ & $\overline{\mathrm{x}}_{6}\left(1+1.5 \delta_{6}\right)$ \\
\hline$\overline{\mathrm{x}}_{1}\left(1+3 \delta_{1}\right)$ & $\overline{\mathrm{x}}_{2}$ & $\overline{\mathrm{x}}_{3}\left(1+1.5 \delta_{3}\right)$ & $\overline{\mathrm{X}}_{4}\left(1+1.5 \delta_{4}\right)$ & $\overline{\mathrm{x}}_{5}\left(1+1.5 \delta_{5}\right)$ & $\overline{\mathrm{x}}_{6}\left(1+1.5 \delta_{6}\right)$ \\
\hline$\overline{\mathrm{x}}_{1}$ & $\overline{\mathrm{x}}_{2}\left(1-3 \delta_{2}\right)$ & $\overline{\mathrm{x}}_{3}\left(1+1.5 \delta_{3}\right)$ & $\overline{\mathrm{X}}_{4}\left(1+1.5 \delta_{4}\right)$ & $\overline{\mathrm{X}}_{5}\left(1+1.5 \delta_{5}\right)$ & $\overline{\mathrm{x}}_{6}\left(1+1.5 \delta_{6}\right)$ \\
\hline$\overline{\mathrm{X}}_{1}$ & $\overline{\mathrm{x}}_{2}\left(1+3 \delta_{2}\right)$ & $\overline{\mathrm{x}}_{3}\left(1+1.5 \delta_{3}\right)$ & $\overline{\mathrm{X}}_{4}\left(1+1.5 \delta_{4}\right)$ & $\overline{\mathrm{X}}_{5}\left(1+1.5 \delta_{5}\right)$ & $\overline{\mathrm{x}}_{6}\left(1+1.5 \delta_{6}\right)$ \\
\hline$\overline{\mathrm{X}}_{1}$ & $\bar{x}_{2}$ & $\overline{\mathrm{x}}_{3}\left(1+1.5 \delta_{3}\right)$ & $\overline{\mathrm{X}}_{4}\left(1+1.5 \delta_{4}\right)$ & $\overline{\mathrm{X}}_{5}\left(1+1.5 \delta_{5}\right)$ & $\overline{\mathrm{x}}_{6}\left(1+1.5 \delta_{6}\right)$ \\
\hline$\overline{\mathrm{x}}_{1}\left(1-3 \delta_{1}\right)$ & $\overline{\mathrm{x}}_{2}$ & $\overline{\mathrm{x}}_{3}$ & $\overline{\mathrm{x}}_{4}\left(1-3 \delta_{4}\right)$ & $\overline{\mathrm{x}}_{5}\left(1+3 \delta_{5}\right)$ & $\overline{\mathrm{x}}_{6}\left(1+3 \delta_{6}\right)$ \\
\hline$\overline{\mathrm{x}}_{1}\left(1+3 \delta_{1}\right)$ & $\overline{\mathrm{x}}_{2}$ & $\overline{\mathrm{X}}_{3}$ & $\overline{\mathrm{X}}_{4}\left(1-3 \delta_{4}\right)$ & $\overline{\mathrm{X}}_{5}\left(1+3 \delta_{5}\right)$ & $\bar{x}_{6}\left(1+3 \delta_{6}\right)$ \\
\hline$\overline{\mathrm{x}}_{1}$ & $\overline{\mathrm{x}}_{2}\left(1-3 \delta_{2}\right)$ & $\overline{\mathrm{x}}_{3}$ & $\overline{\mathrm{x}}_{4}\left(1-3 \delta_{4}\right)$ & $\overline{\mathrm{x}}_{5}\left(1+3 \delta_{5}\right)$ & $\overline{\mathrm{x}}_{6}\left(1+3 \delta_{6}\right)$ \\
\hline$\overline{\mathrm{x}}_{1}$ & $\overline{\mathrm{x}}_{2}\left(1+3 \delta_{2}\right)$ & $\overline{\mathrm{x}}_{3}$ & $\overline{\mathrm{x}}_{4}\left(1-3 \delta_{4}\right)$ & $\overline{\mathrm{X}}_{5}\left(1+3 \delta_{5}\right)$ & $\bar{x}_{6}\left(1+3 \delta_{6}\right)$ \\
\hline$\overline{\mathrm{x}}_{1}$ & $\overline{\mathrm{x}}_{2}$ & $\overline{\mathrm{x}}_{3}$ & $\overline{\mathrm{x}}_{4}\left(1-3 \delta_{4}\right)$ & $\overline{\mathrm{X}}_{5}\left(1+3 \delta_{5}\right)$ & $\overline{\mathrm{x}}_{6}\left(1+3 \delta_{6}\right)$ \\
\hline$\overline{\mathrm{x}}_{1}\left(1-3 \delta_{1}\right)$ & $\overline{\mathrm{x}}_{2}$ & $\overline{\mathrm{x}}_{3}$ & $\overline{\mathrm{x}}_{4}\left(1-3 \delta_{4}\right)$ & $\overline{\mathrm{X}}_{5}\left(1+3 \delta_{5}\right)$ & $\overline{\mathrm{x}}_{6}\left(1+3 \delta_{6}\right)$ \\
\hline
\end{tabular}

\subsubsection{Plano de Experiência Numérico Hiper-cubo ou}

\section{Estrela 45}

Este plano de experiência equivale ao plano de experiência estrela rotacionado de 45 graus.

Os coeficientes propostos para problemas com 1 variável aleatória são:

$$
\left\{\begin{array}{lll}
\bar{x}_{1} & \overline{\mathrm{x}}_{1}\left(1-3 \delta_{1}\right) & \overline{\mathrm{x}}_{1}\left(1+3 \delta_{1}\right.
\end{array}\right\}
$$


Os coeficientes propostos para problemas com 2 variáveis aleatórias são:

$$
\left[\begin{array}{cc}
\bar{x}_{1} & \bar{x}_{2} \\
\bar{x}_{1}\left(1+3 \delta_{1}\right) & \bar{x}_{2}\left(1+3 \delta_{2}\right) \\
\bar{x}_{1}\left(1-3 \delta_{1}\right) & \bar{x}_{2}\left(1+3 \delta_{2}\right) \\
\bar{x}_{1}\left(1-3 \delta_{1}\right) & \bar{x}_{2}\left(1-3 \delta_{2}\right) \\
\bar{x}_{1}\left(1+3 \delta_{1}\right) & \bar{x}_{2}\left(1-3 \delta_{2}\right)
\end{array}\right]
$$

Os coeficientes propostos para problemas com 3 variáveis aleatórias são:

$$
\left[\begin{array}{ccc}
\overline{\mathrm{x}}_{1} & \overline{\mathrm{x}}_{2} & \overline{\mathrm{x}}_{3} \\
\overline{\mathrm{x}}_{1}\left(1+3 \delta_{1}\right) & \overline{\mathrm{x}}_{2}\left(1+3 \delta_{2}\right) & \overline{\mathrm{x}}_{3}\left(1-3 \delta_{3}\right) \\
\overline{\mathrm{x}}_{1}\left(1-3 \delta_{1}\right) & \overline{\mathrm{x}}_{2}\left(1+3 \delta_{2}\right) & \overline{\mathrm{x}}_{3}\left(1-3 \delta_{3}\right) \\
\overline{\mathrm{x}}_{1}\left(1-3 \delta_{1}\right) & \overline{\mathrm{x}}_{2}\left(1-3 \delta_{2}\right) & \overline{\mathrm{x}}_{3}\left(1-3 \delta_{3}\right) \\
\overline{\mathrm{x}}_{1}\left(1+3 \delta_{1}\right) & \overline{\mathrm{x}}_{2}\left(1-3 \delta_{2}\right) & \overline{\mathrm{x}}_{3}\left(1-3 \delta_{3}\right) \\
\overline{\mathrm{x}}_{1} & \overline{\mathrm{x}}_{2} & \overline{\mathrm{x}}_{3}\left(1+3 \delta_{3}\right) \\
\overline{\mathrm{x}}_{1}\left(1+3 \delta_{1}\right) & \overline{\mathrm{x}}_{2}\left(1+3 \delta_{2}\right) & \overline{\mathrm{x}}_{3}\left(1+3 \delta_{3}\right) \\
\overline{\mathrm{x}}_{1}\left(1-3 \delta_{1}\right) & \overline{\mathrm{x}}_{2}\left(1+3 \delta_{2}\right) & \overline{\mathrm{x}}_{3}\left(1+3 \delta_{3}\right) \\
\overline{\mathrm{x}}_{1}\left(1-3 \delta_{1}\right) & \overline{\mathrm{x}}_{2}\left(1-3 \delta_{2}\right) & \overline{\mathrm{x}}_{3}\left(1+3 \delta_{3}\right)
\end{array}\right]
$$

Os coeficientes propostos para problemas com 4 variáveis aleatórias são: 


$\left[\begin{array}{cccc}\overline{\mathrm{x}}_{1} & \overline{\mathrm{x}}_{2} & \overline{\mathrm{x}}_{3} & \overline{\mathrm{x}}_{4} \\ \overline{\mathrm{x}}_{1}\left(1+3 \delta_{1}\right) & \overline{\mathrm{x}}_{2}\left(1+3 \delta_{2}\right) & \overline{\mathrm{x}}_{3}\left(1-3 \delta_{3}\right) & \overline{\mathrm{x}}_{4}\left(1-3 \delta_{4}\right) \\ \overline{\mathrm{x}}_{1}\left(1-3 \delta_{1}\right) & \overline{\mathrm{x}}_{2}\left(1+3 \delta_{2}\right) & \overline{\mathrm{x}}_{3}\left(1-3 \delta_{3}\right) & \overline{\mathrm{x}}_{4}\left(1-3 \delta_{4}\right) \\ \overline{\mathrm{x}}_{1}\left(1-3 \delta_{1}\right) & \overline{\mathrm{x}}_{2}\left(1-3 \delta_{2}\right) & \overline{\mathrm{x}}_{3}\left(1-3 \delta_{3}\right) & \overline{\mathrm{x}}_{4}\left(1-3 \delta_{4}\right) \\ \overline{\mathrm{x}}_{1}\left(1+3 \delta_{1}\right) & \overline{\mathrm{x}}_{2}\left(1-3 \delta_{2}\right) & \overline{\mathrm{x}}_{3}\left(1-3 \delta_{3}\right) & \overline{\mathrm{x}}_{4}\left(1-3 \delta_{4}\right) \\ \overline{\mathrm{x}}_{1} & \overline{\mathrm{x}}_{2} & \overline{\mathrm{x}}_{3}\left(1+3 \delta_{3}\right) & \overline{\mathrm{x}}_{4}\left(1+3 \delta_{4}\right) \\ \overline{\mathrm{x}}_{1}\left(1+3 \delta_{1}\right) & \overline{\mathrm{x}}_{2}\left(1+3 \delta_{2}\right) & \overline{\mathrm{x}}_{3}\left(1+3 \delta_{3}\right) & \overline{\mathrm{x}}_{4}\left(1+3 \delta_{4}\right) \\ \overline{\mathrm{x}}_{1}\left(1-3 \delta_{1}\right) & \overline{\mathrm{x}}_{2}\left(1+3 \delta_{2}\right) & \overline{\mathrm{x}}_{3}\left(1+3 \delta_{3}\right) & \overline{\mathrm{x}}_{4}\left(1+3 \delta_{4}\right) \\ \overline{\mathrm{x}}_{1}\left(1-3 \delta_{1}\right) & \overline{\mathrm{x}}_{2}\left(1-3 \delta_{2}\right) & \overline{\mathrm{x}}_{3}\left(1+3 \delta_{3}\right) & \overline{\mathrm{x}}_{4}\left(1+3 \delta_{4}\right) \\ \overline{\mathrm{x}}_{1}\left(1+3 \delta_{1}\right) & \overline{\mathrm{x}}_{2}\left(1-3 \delta_{2}\right) & \overline{\mathrm{x}}_{3}\left(1+3 \delta_{3}\right) & \overline{\mathrm{x}}_{4}\left(1+3 \delta_{4}\right) \\ \overline{\mathrm{x}}_{1} & \overline{\mathrm{x}}_{2} & \overline{\mathrm{x}}_{3}\left(1-1.5 \delta_{3}\right) & \overline{\mathrm{x}}_{4}\left(1-1.5 \delta_{4}\right) \\ \overline{\mathrm{x}}_{1}\left(1+3 \delta_{1}\right) & \overline{\mathrm{x}}_{2}\left(1+3 \delta_{2}\right) & \overline{\mathrm{x}}_{3}\left(1-1.5 \delta_{3}\right) & \overline{\mathrm{x}}_{4}\left(1-1.5 \delta_{4}\right) \\ \overline{\mathrm{x}}_{1}\left(1-3 \delta_{1}\right) & \overline{\mathrm{x}}_{2}\left(1+3 \delta_{2}\right) & \overline{\mathrm{x}}_{3}\left(1-1.5 \delta_{3}\right) & \overline{\mathrm{x}}_{4}\left(1-1.5 \delta_{4}\right) \\ \overline{\mathrm{x}}_{1}\left(1-3 \delta_{1}\right) & \overline{\mathrm{x}}_{2}\left(1-3 \delta_{2}\right) & \overline{\mathrm{x}}_{3}\left(1-1.5 \delta_{3}\right) & \overline{\mathrm{x}}_{4}\left(1-1.5 \delta_{4}\right)\end{array}\right]$

Os coeficientes propostos para problemas com 5 variáveis aleatórias são:

\begin{tabular}{|c|c|c|c|c|}
\hline & & & & \\
\hline $\mathrm{X}_{1}$ & $X_{2}$ & $X_{3}$ & $\mathrm{X}_{4}$ & $X_{5}$ \\
\hline $\bar{x}_{1}\left(1+3 \delta_{1}\right)$ & $\bar{x}_{2}\left(1+3 \delta_{2}\right)$ & $\bar{x}_{3}\left(1-3 \delta_{3}\right)$ & $\overline{\mathrm{X}}_{4}\left(1-3 \delta_{4}\right)$ & $\bar{X}_{5}\left(1-3 \delta_{5}\right)$ \\
\hline $\bar{x}_{1}\left(1-3 \delta_{1}\right)$ & $\bar{x}_{2}\left(1+3 \delta_{2}\right)$ & $\bar{x}_{3}\left(1-3 \delta_{3}\right)$ & $\bar{x}_{4}\left(1-3 \delta_{4}\right)$ & $\bar{x}_{5}\left(1-3 \delta_{5}\right)$ \\
\hline $\mathrm{X}_{1}\left(1-3 \delta_{1}\right)$ & $\bar{x}_{2}\left(1-3 \delta_{2}\right)$ & $\bar{x}_{3}\left(1-3 \delta_{3}\right)$ & $\bar{x}_{4}\left(1-3 \delta_{4}\right)$ & $\bar{x}_{5}\left(1-3 \delta_{5}\right)$ \\
\hline $\bar{x}_{1}\left(1+3 \delta_{1}\right)$ & $\bar{x}_{2}\left(1-3 \delta_{2}\right)$ & $\bar{x}_{3}\left(1-3 \delta_{3}\right)$ & $\bar{x}_{4}\left(1-3 \delta_{4}\right)$ & $\bar{x}_{5}\left(1-3 \delta_{5}\right)$ \\
\hline $\bar{x}_{1}$ & $\bar{x}_{2}$ & $\bar{x}_{3}\left(1-3 \delta_{3}\right)$ & $\bar{x}_{4}\left(1-3 \delta_{4}\right)$ & $\bar{x}_{5}\left(1-3 \delta_{5}\right)$ \\
\hline $\bar{x}_{1}\left(1+3 \delta_{1}\right)$ & $\bar{x}_{2}\left(1+3 \delta_{2}\right)$ & $\bar{x}_{3}\left(1+3 \delta_{3}\right)$ & $\bar{x}_{4}\left(1+3 \delta_{4}\right)$ & $\bar{x}_{5}\left(1+3 \delta_{5}\right)$ \\
\hline $\bar{x}_{1}\left(1-3 \delta_{1}\right)$ & $\bar{x}_{2}\left(1+3 \delta_{2}\right)$ & $\bar{x}_{3}\left(1+3 \delta_{3}\right)$ & $\bar{x}_{4}\left(1+3 \delta_{4}\right)$ & $\bar{x}_{5}\left(1+3 \delta_{5}\right)$ \\
\hline $\bar{x}_{1}\left(1-3 \delta_{1}\right)$ & $\bar{x}_{2}\left(1-3 \delta_{2}\right)$ & $\bar{x}_{3}\left(1+3 \delta_{3}\right)$ & $\bar{x}_{4}\left(1+3 \delta_{4}\right)$ & $\bar{x}_{5}\left(1+3 \delta_{5}\right)$ \\
\hline $\bar{x}_{1}\left(1+3 \delta_{1}\right)$ & $\bar{x}_{2}\left(1-3 \delta_{2}\right)$ & $\bar{x}_{3}\left(1+3 \delta_{3}\right)$ & $\bar{x}_{4}\left(1+3 \delta_{4}\right)$ & $\bar{x}_{5}\left(1+3 \delta_{5}\right)$ \\
\hline $\bar{x}_{1}$ & $\bar{x}_{2}$ & $\bar{x}_{3}\left(1+3 \delta_{3}\right)$ & $\bar{x}_{4}\left(1+3 \delta_{4}\right)$ & $\bar{x}_{5}\left(1+3 \delta_{5}\right)$ \\
\hline $\mathrm{x}_{1}\left(1+3 \delta_{1}\right)$ & $\bar{x}_{2}\left(1+3 \delta_{2}\right)$ & $\bar{x}_{3}\left(1-1.5 \delta_{3}\right)$ & $\bar{x}_{4}\left(1-1.5 \delta_{4}\right)$ & $\bar{x}_{5}\left(1-1.5 \delta_{5}\right)$ \\
\hline $\bar{x}_{1}\left(1-3 \delta_{1}\right)$ & $\bar{x}_{2}\left(1+3 \delta_{2}\right)$ & $\bar{x}_{3}\left(1-1.5 \delta_{3}\right)$ & $\bar{x}_{4}\left(1-1.5 \delta_{4}\right)$ & $\bar{x}_{5}\left(1-1.5 \delta_{5}\right)$ \\
\hline $\bar{x}_{1}\left(1-3 \delta_{1}\right)$ & $\bar{x}_{2}\left(1-3 \delta_{2}\right)$ & $\bar{x}_{3}\left(1-1.5 \delta_{3}\right)$ & $\bar{x}_{4}\left(1-1.5 \delta_{4}\right)$ & $\bar{x}_{5}\left(1-1.5 \delta_{5}\right)$ \\
\hline $\bar{x}_{1}\left(1+3 \delta_{1}\right)$ & $\bar{x}_{2}\left(1-3 \delta_{2}\right)$ & $\bar{x}_{3}\left(1-1.5 \delta_{3}\right)$ & $\bar{x}_{4}\left(1-1.5 \delta_{4}\right)$ & $\bar{x}_{5}\left(1-1.5 \delta_{5}\right)$ \\
\hline $\bar{x}_{1}$ & $\bar{x}_{2}$ & $\mathrm{x}_{3}\left(1-1.5 \delta_{3}\right)$ & $\bar{x}_{4}\left(1-1.5 \delta_{14}\right)$ & $\mathrm{X}_{5}\left(1-1.5 \delta_{5}\right)$ \\
\hline $\bar{x}_{1}\left(1+3 \delta_{1}\right)$ & $\bar{x}_{2}\left(1+3 \delta_{2}\right)$ & $\bar{x}_{3}\left(1+1.5 \delta_{3}\right)$ & $\bar{x}_{4}\left(1+1.5 \delta_{4}\right)$ & $\bar{x}_{5}\left(1+1.5 \delta_{5}\right)$ \\
\hline $\bar{x}_{1}\left(1-3 \delta_{1}\right)$ & $\mathrm{x}_{2}\left(1+3 \delta_{2}\right)$ & $\bar{x}_{3}\left(1+1.5 \delta_{3}\right)$ & $\mathrm{x}_{4}\left(1+1.5 \delta_{4}\right)$ & $\mathrm{X}_{5}\left(1+1.5 \delta_{5}\right)$ \\
\hline $\bar{x}_{1}\left(1-3 \delta_{1}\right)$ & $\bar{x}_{2}\left(1-3 \delta_{2}\right)$ & $\bar{x}_{3}\left(1+1.5 \delta_{3}\right)$ & $\bar{x}_{4}\left(1+1.5 \delta_{4}\right)$ & $\bar{x}_{5}\left(1+1.5 \delta_{5}\right)$ \\
\hline $\bar{x}_{1}\left(1+3 \delta_{1}\right)$ & $\mathrm{x}_{2}\left(1-3 \delta_{2}\right)$ & $\bar{x}_{3}\left(1+1.5 \delta_{3}\right)$ & $\mathrm{x}_{4}\left(1+1.5 \delta_{4}\right)$ & $\bar{x}_{5}\left(1+1.5 \delta_{5}\right)$ \\
\hline
\end{tabular}


Os coeficientes propostos para problemas com 6 variáveis aleatórias são:

\begin{tabular}{|c|c|c|c|c|c|}
\hline $\mathrm{X}_{1}$ & $\mathrm{X}_{2}$ & $\mathrm{x}_{3}$ & $\mathrm{X}_{4}$ & $X_{5}$ & $X_{6}$ \\
\hline $\bar{x}_{1}\left(1+3 \delta_{1}\right)$ & $\bar{x}_{2}\left(1+3 \delta_{2}\right)$ & $\bar{x}_{3}\left(1-3 \delta_{3}\right)$ & $\bar{x}_{4}\left(1-3 \delta_{4}\right)$ & $\bar{x}_{5}\left(1-3 \delta_{5}\right)$ & $\bar{x}_{6}\left(1-3 \delta_{6}\right)$ \\
\hline $\bar{x}_{1}\left(1-3 \delta_{1}\right)$ & $\bar{x}_{2}\left(1+3 \delta_{2}\right)$ & $\bar{x}_{3}\left(1-3 \delta_{3}\right)$ & $\overline{\mathrm{X}}_{4}\left(1-3 \delta_{4}\right)$ & $\bar{x}_{5}\left(1-3 \delta_{5}\right)$ & $\bar{x}_{6}\left(1-3 \delta_{6}\right)$ \\
\hline $\bar{x}_{1}\left(1-3 \delta_{1}\right)$ & $\bar{x}_{2}\left(1-3 \delta_{2}\right)$ & $\bar{x}_{3}\left(1-3 \delta_{3}\right)$ & $\bar{x}_{4}\left(1-3 \delta_{4}\right)$ & $\bar{x}_{5}\left(1-3 \delta_{5}\right)$ & $\bar{x}_{6}\left(1-3 \delta_{6}\right)$ \\
\hline$\overline{\mathrm{x}}_{1}\left(1+3 \delta_{1}\right)$ & $\bar{x}_{2}\left(1-3 \delta_{2}\right)$ & $\overline{\mathrm{x}}_{3}\left(1-3 \delta_{3}\right)$ & $\overline{\mathrm{X}}_{4}\left(1-3 \delta_{4}\right)$ & $\bar{x}_{5}\left(1-3 \delta_{5}\right)$ & $\bar{x}_{6}\left(1-3 \delta_{6}\right)$ \\
\hline $\bar{x}_{1}$ & $\bar{x}_{2}$ & $\bar{x}_{3}\left(1-3 \delta_{3}\right)$ & $\bar{x}_{4}\left(1-3 \delta_{4}\right)$ & $\bar{x}_{5}\left(1-3 \delta_{5}\right)$ & $\bar{x}_{6}\left(1-3 \delta_{6}\right)$ \\
\hline$\overline{\mathrm{x}}_{1}\left(1+3 \delta_{1}\right)$ & $\overline{\mathrm{x}}_{2}\left(1+3 \delta_{2}\right)$ & $\bar{x}_{3}\left(1+3 \delta_{3}\right)$ & $\overline{\mathrm{X}}_{4}\left(1+3 \delta_{4}\right)$ & $\bar{x}_{5}\left(1+3 \delta_{5}\right)$ & $\bar{x}_{6}\left(1+3 \delta_{6}\right)$ \\
\hline $\bar{x}_{1}\left(1-3 \delta_{1}\right)$ & $\mathrm{x}_{2}\left(1+3 \delta_{2}\right)$ & $\mathrm{x}_{3}\left(1+3 \delta_{3}\right)$ & $\mathrm{X}_{4}\left(1+3 \delta_{4}\right)$ & $\mathrm{x}_{5}\left(1+3 \delta_{5}\right)$ & $\mathrm{x}_{6}\left(1+3 \delta_{6}\right)$ \\
\hline$\overline{\mathrm{x}}_{1}\left(1-3 \delta_{1}\right)$ & $\overline{\mathrm{x}}_{2}\left(1-3 \delta_{2}\right)$ & $\bar{x}_{3}\left(1+3 \delta_{3}\right)$ & $\overline{\mathrm{X}}_{4}\left(1+3 \delta_{4}\right)$ & $\overline{\mathrm{X}}_{5}\left(1+3 \delta_{5}\right)$ & $\overline{\mathrm{X}}_{6}\left(1+3 \delta_{6}\right)$ \\
\hline $\bar{x}_{1}\left(1+3 \delta_{1}\right)$ & $\bar{x}_{2}\left(1-3 \delta_{2}\right)$ & $\bar{x}_{3}\left(1+3 \delta_{3}\right)$ & $\bar{x}_{4}\left(1+3 \delta_{4}\right)$ & $\bar{x}_{5}\left(1+3 \delta_{5}\right)$ & $\bar{x}_{6}\left(1+3 \delta_{6}\right)$ \\
\hline $\bar{x}_{1}$ & $\overline{\mathrm{X}}_{2}$ & $\overline{\mathrm{x}}_{3}\left(1+3 \delta_{3}\right)$ & $\overline{\mathrm{X}}_{4}\left(1+3 \delta_{4}\right)$ & $\overline{\mathrm{X}}_{5}\left(1+3 \delta_{5}\right)$ & $\overline{\mathrm{x}}_{6}\left(1+3 \delta_{6}\right)$ \\
\hline $\bar{x}_{1}\left(1+3 \delta_{1}\right)$ & $\bar{x}_{2}\left(1+3 \delta_{2}\right)$ & $\bar{x}_{3}\left(1-1.5 \delta_{3}\right)$ & $\bar{x}_{4}\left(1-1.5 \delta_{4}\right)$ & $\bar{x}_{5}\left(1-1.5 \delta_{5}\right)$ & $\bar{x}_{6}\left(1-1.5 \delta_{6}\right)$ \\
\hline $\bar{x}_{1}\left(1-3 \delta_{1}\right)$ & $\overline{\mathrm{X}}_{2}\left(1+3 \delta_{2}\right)$ & $\overline{\mathrm{x}}_{3}\left(1-1.5 \delta_{3}\right)$ & $\bar{x}_{4}\left(1-1.5 \delta_{4}\right)$ & $\bar{x}_{5}\left(1-1.5 \delta_{5}\right)$ & $\bar{x}_{6}\left(1-1.5 \delta_{6}\right)$ \\
\hline$\overline{\mathrm{x}}_{1}\left(1-3 \delta_{1}\right)$ & $\bar{x}_{2}\left(1-3 \delta_{2}\right)$ & $\bar{x}_{3}\left(1-1.5 \delta_{3}\right)$ & $\overline{\mathrm{x}}_{4}\left(1-1.5 \delta_{4}\right)$ & $\bar{x}_{5}\left(1-1.5 \delta_{5}\right)$ & $\bar{x}_{6}\left(1-1.5 \delta_{6}\right)$ \\
\hline$x_{1}\left(1+3 \delta_{1}\right)$ & $\mathrm{x}_{2}\left(1-3 \delta_{2}\right)$ & $x_{3}\left(1-1.5 \delta_{3}\right)$ & $x_{4}\left(1-1.5 \delta_{4}\right)$ & $x_{5}\left(1-1.5 \delta_{5}\right)$ & $\mathrm{X}_{6}\left(1-1.5 \delta_{6}\right)$ \\
\hline $\bar{x}_{1}$ & $\bar{x}_{2}$ & $\bar{x}_{3}\left(1-1.5 \delta_{3}\right)$ & $\bar{x}_{4}\left(1-1.5 \delta_{4}\right)$ & $\bar{x}_{5}\left(1-1.5 \delta_{5}\right)$ & $\bar{x}_{6}\left(1-1.5 \delta_{6}\right)$ \\
\hline $\bar{x}_{1}\left(1+3 \delta_{1}\right)$ & $\overline{\mathrm{X}}_{2}\left(1+3 \delta_{2}\right)$ & $\bar{x}_{3}\left(1+1.5 \delta_{3}\right)$ & $\bar{x}_{4}\left(1+1.5 \delta_{4}\right)$ & $\bar{x}_{5}\left(1+1.5 \delta_{5}\right)$ & $\bar{x}_{6}\left(1+1.5 \delta_{6}\right)$ \\
\hline $\bar{x}_{1}\left(1-3 \delta_{1}\right)$ & $\bar{x}_{2}\left(1+3 \delta_{2}\right)$ & $\bar{x}_{3}\left(1+1.5 \delta_{3}\right)$ & $\bar{x}_{4}\left(1+1.5 \delta_{4}\right)$ & $\bar{x}_{5}\left(1+1.5 \delta_{5}\right)$ & $\bar{x}_{6}\left(1+1.5 \delta_{6}\right)$ \\
\hline$x_{1}\left(1-3 \delta_{1}\right)$ & $x_{2}\left(1-3 \delta_{2}\right)$ & $x_{3}\left(1+1.5 \delta_{3}\right)$ & $x_{4}\left(1+1.5 \delta_{4}\right)$ & $x_{5}\left(1+1.5 \delta_{5}\right)$ & $x_{6}\left(1+1.5 \delta_{6}\right)$ \\
\hline $\bar{x}_{1}\left(1+3 \delta_{1}\right)$ & $\bar{x}_{2}\left(1-3 \delta_{2}\right)$ & $\bar{x}_{3}\left(1+1.5 \delta_{3}\right)$ & $\bar{x}_{4}\left(1+1.5 \delta_{4}\right)$ & $\bar{x}_{5}\left(1+1.5 \delta_{5}\right)$ & $\bar{x}_{6}\left(1+1.5 \delta_{6}\right)$ \\
\hline $\bar{x}_{1}$ & $\bar{x}_{2}$ & $\bar{x}_{3}\left(1+1.5 \delta_{3}\right)$ & $\overline{\mathrm{x}}_{4}\left(1+1.5 \delta_{4}\right)$ & $\bar{x}_{5}\left(1+1.5 \delta_{5}\right)$ & $\bar{x}_{6}\left(1+1.5 \delta_{6}\right)$ \\
\hline $\bar{x}_{1}\left(1+3 \delta_{1}\right)$ & $\bar{x}_{2}\left(1+3 \delta_{2}\right)$ & $\bar{x}_{3}$ & $\bar{x}_{4}\left(1-3 \delta_{4}\right)$ & $\bar{x}_{5}\left(1+3 \delta_{5}\right)$ & $\bar{x}_{6}\left(1+3 \delta_{6}\right)$ \\
\hline$x_{1}\left(1-3 \delta_{1}\right)$ & $x_{2}\left(1+3 \delta_{2}\right)$ & $x_{3}$ & $x_{4}\left(1-3 \delta_{4}\right)$ & $x_{5}\left(1+3 \delta_{5}\right)$ & $\mathrm{x}_{6}\left(1+3 \delta_{6}\right)$ \\
\hline $\bar{x}_{1}\left(1-3 \delta_{1}\right)$ & $\bar{x}_{2}\left(1-3 \delta_{2}\right)$ & $\bar{x}_{3}$ & $\bar{x}_{4}\left(1-3 \delta_{4}\right)$ & $\bar{x}_{5}\left(1+3 \delta_{5}\right)$ & $\bar{x}_{6}\left(1+3 \delta_{6}\right)$ \\
\hline $\bar{x}_{1}\left(1+3 \delta_{1}\right)$ & $\bar{x}_{2}\left(1-3 \delta_{2}\right)$ & $\bar{x}_{3}$ & $\bar{x}_{4}\left(1-3 \delta_{4}\right)$ & $\bar{x}_{5}\left(1+3 \delta_{5}\right)$ & $\bar{x}_{6}\left(1+3 \delta_{6}\right)$ \\
\hline $\bar{x}_{1}$ & $\bar{x}_{2}$ & $\bar{x}_{3}$ & $\bar{x}_{4}\left(1-3 \delta_{4}\right)$ & $\bar{x}_{5}\left(1+3 \delta_{5}\right)$ & $\bar{x}_{6}\left(1+3 \delta_{6}\right)$ \\
\hline $\mathrm{x}_{1}\left(1+3 \delta_{1}\right)$ & $\mathrm{x}_{2}\left(1+3 \delta_{2}\right)$ & $x_{3}$ & $x_{4}\left(1-3 \delta_{4}\right)$ & $\mathrm{x}_{5}\left(1+3 \delta_{5}\right)$ & $\mathrm{x}_{6}\left(1+3 \delta_{6}\right)$ \\
\hline
\end{tabular}

\subsubsection{Plano de Experiência Numérico Fatorial Completo}

Como se pode observar esse plano de experiência exige um número bem maior de pontos do que o número de coeficientes do polinômio aproximador da SR. A princípio pode-se pensar que planos com essas 
características podem não ser adequados devido ao aumento do custo computacional exigido. Entretanto, há estruturas que não levam a convergência quando se utilizam planos com um número menor de pontos. Como já foi comentado, cada caso deve ser analisado isoladamente, podendo apresentar convergência mais rápida mesmo quando o número de pontos inicial é maior.

Os coeficientes propostos para problemas com 1 variável aleatória são:

$$
\left\{\begin{array}{lll}
\bar{x}_{1} & \bar{x}_{1}\left(1-3 \delta_{1}\right) & \bar{x}_{1}\left(1+3 \delta_{1}\right.
\end{array}\right\}
$$

Os coeficientes propostos para problemas com 2 ou mais variáveis aleatórias, v_x, são expressos de forma generalizada na Equação (5.43).

$$
v_{-} x(i, j)=C(L(i, j), j) \quad \backslash \quad i=1, \ldots, n_{p r} ; \quad j=1, \ldots, n_{v}
$$

A matriz C é representada pela Equação (5.44):

$$
\mathrm{C}=\left[\begin{array}{cccc}
\overline{\mathrm{x}}_{1}\left(1-3 \delta_{1}\right) & \overline{\mathrm{x}}_{2}\left(1-3 \delta_{2}\right) & \ldots & \overline{\mathrm{x}}_{\mathrm{n}_{\mathrm{v}}}\left(1-3 \delta_{\mathrm{n}_{\mathrm{v}}}\right) \\
\overline{\mathrm{x}}_{1}\left(3 \delta_{1}\right) & \overline{\mathrm{x}}_{2}\left(3 \delta_{2}\right) & \ldots & \overline{\mathrm{x}}_{\mathrm{n}_{\mathrm{v}}}\left(3 \delta_{\mathrm{n}_{\mathrm{v}}}\right) \\
\overline{\mathrm{x}}_{1}\left(1+3 \delta_{1}\right) & \overline{\mathrm{x}}_{2}\left(1+3 \delta_{2}\right) & \ldots & \overline{\mathrm{x}}_{\mathrm{n}_{\mathrm{v}}}\left(1+3 \delta_{\mathrm{n}_{\mathrm{v}}}\right)
\end{array}\right]
$$

onde $\mathrm{n}_{\mathrm{v}}$ refere-se ao número de variáveis aleatórias e $\mathrm{n}_{\mathrm{pr}} \mathrm{O}$ número de pontos do plano de experiência. No caso,

$$
\mathrm{n}_{\mathrm{pr}}=3\left(3^{\left(\mathrm{n}_{\mathrm{v}}-1\right)}\right)
$$

sendo a matriz L é representada pela Equação (5.46): 


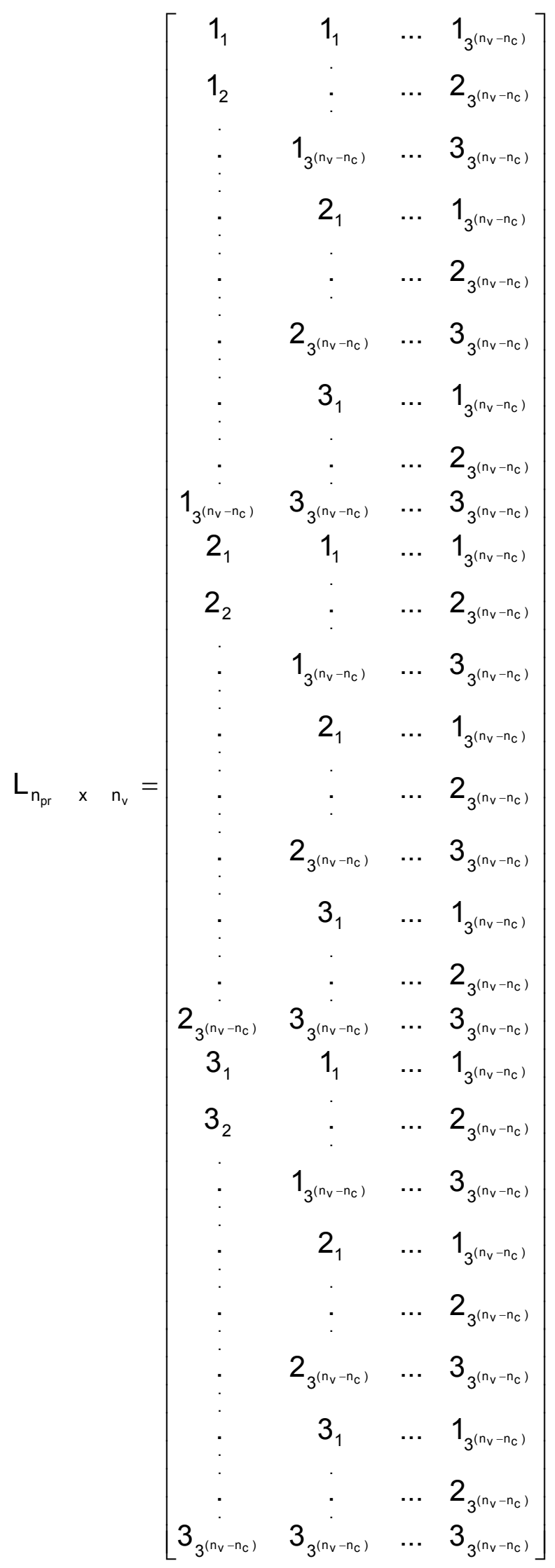




\subsubsection{Plano de Experiência Numérico Mínimo}

Esse plano de experiência é similar ao plano Estrela, diferenciando-se pela presença de pontos adicionais de tal forma que o número total de pontos é igual ao número de coeficientes do polinômio aproximador da SR.

Os coeficientes propostos para problemas com 1 variável aleatória são:

$$
\left\{\begin{array}{lll}
\bar{x}_{1} & \bar{x}_{1}\left(1-3 \delta_{1}\right) & \bar{x}_{1}\left(1+3 \delta_{1}\right.
\end{array}\right\}
$$

Os coeficientes propostos para problemas com 2 variáveis aleatórias são:

$$
\left[\begin{array}{cc}
\bar{x}_{1} & \bar{x}_{2} \\
\bar{x}_{1}\left(1-3 \delta_{1}\right) & \bar{x}_{2} \\
\bar{x}_{1}\left(1+3 \delta_{1}\right) & \bar{x}_{2} \\
\bar{x}_{1} & \bar{x}_{2}\left(1-3 \delta_{2}\right) \\
\bar{x}_{1} & \bar{x}_{2}\left(1+3 \delta_{2}\right) \\
\bar{x}_{1}\left(1+3 \delta_{1}\right) & \bar{x}_{2}\left(1+3 \delta_{2}\right)
\end{array}\right]
$$

Os coeficientes propostos para problemas com 3 variáveis aleatórias são:

$$
\left[\begin{array}{ccc}
\bar{x}_{1} & \bar{x}_{2} & \bar{x}_{3} \\
\bar{x}_{1}\left(1-3 \delta_{1}\right) & \bar{x}_{2} & \bar{x}_{3}\left(1-3 \delta_{3}\right) \\
\bar{x}_{1}\left(1+3 \delta_{1}\right) & \bar{x}_{2} & \bar{x}_{3}\left(1-3 \delta_{3}\right) \\
\bar{x}_{1} & \bar{x}_{2}\left(1-3 \delta_{2}\right) & \bar{x}_{3}\left(1-3 \delta_{3}\right) \\
\bar{x}_{1} & \bar{x}_{2}\left(1+3 \delta_{2}\right) & \bar{x}_{3}\left(1-3 \delta_{3}\right) \\
\bar{x}_{1}\left(1+3 \delta_{1}\right) & \bar{x}_{2}\left(1+3 \delta_{2}\right) & \bar{x}_{3}\left(1+3 \delta_{3}\right) \\
\bar{x}_{1} & \bar{x}_{2} & \bar{x}_{3}\left(1+3 \delta_{3}\right) \\
\bar{x}_{1}\left(1-3 \delta_{1}\right) & \bar{x}_{2} & \bar{x}_{3}\left(1+3 \delta_{3}\right) \\
\bar{x}_{1}\left(1+3 \delta_{1}\right) & \bar{x}_{2} & \bar{x}_{3}\left(1+3 \delta_{3}\right) \\
\bar{x}_{1} & \bar{x}_{2}\left(1-3 \delta_{2}\right) & \bar{x}_{3}\left(1+3 \delta_{3}\right)
\end{array}\right]
$$


Os coeficientes propostos para problemas com 4 variáveis aleatórias são:

$$
\left[\begin{array}{cccc}
\bar{x}_{1} & \bar{x}_{2} & \bar{x}_{3} & \bar{x}_{4} \\
\bar{x}_{1}\left(1-3 \delta_{1}\right) & \bar{x}_{2} & \bar{x} & \bar{x}_{4}\left(1-3 \delta_{4}\right) \\
\bar{x}_{1}\left(1+3 \delta_{1}\right) & \bar{x}_{2} & \bar{x} & \bar{x}_{4}\left(1-3 \delta_{4}\right) \\
\bar{x}_{1} & \bar{x}_{2}\left(1-3 \delta_{2}\right) & \bar{x} & \bar{x}_{4}\left(1-3 \delta_{4}\right) \\
\bar{x}_{1} & \bar{x}_{2}\left(1+3 \delta_{2}\right) & \bar{x} & \bar{x}_{4}\left(1-3 \delta_{4}\right) \\
\bar{x}_{1}\left(1+3 \delta_{1}\right) & \bar{x}_{2}\left(1+3 \delta_{2}\right) & \bar{x} & \bar{x}_{4}\left(1+3 \delta_{4}\right) \\
\overline{x_{1}} & \bar{x}_{2} & \bar{x}_{3}\left(1-3 \delta_{3}\right) & \bar{x}_{4}\left(1+3 \delta_{4}\right) \\
\bar{x}_{1}\left(1-3 \delta_{1}\right) & \bar{x}_{2} & \bar{x}_{3}\left(1-3 \delta_{3}\right) & \bar{x}_{4}\left(1+3 \delta_{4}\right) \\
\bar{x}_{1}\left(1+3 \delta_{1}\right) & \bar{x}_{2} & \bar{x}_{3}\left(1-3 \delta_{3}\right) & \bar{x}_{4}\left(1+3 \delta_{4}\right) \\
\bar{x} & \bar{x}_{2}\left(1-3 \delta_{2}\right) & \bar{x}_{3}\left(1-3 \delta_{3}\right) & \bar{x}_{4}\left(1+3 \delta_{4}\right) \\
\bar{x}_{1} & \bar{x}_{2}\left(1+3 \delta_{2}\right) & \bar{x}_{3}\left(1+3 \delta_{3}\right) & \bar{x}_{4}\left(1-1.5 \delta_{4}\right) \\
\bar{x}_{1}\left(1+3 \delta_{1}\right) & \bar{x}_{2}\left(1+3 \delta_{2}\right) & \bar{x}_{3}\left(1+3 \delta_{3}\right) & \bar{x}_{4}\left(1-1.5 \delta_{4}\right) \\
\bar{x}_{1} & \bar{x}_{2} & \bar{x}_{3}\left(1+3 \delta_{3}\right) & \bar{x}_{4}\left(1-1.5 \delta_{4}\right) \\
\bar{x}_{1}\left(1-3 \delta_{1}\right) & \bar{x}_{2} & \bar{x}_{3}\left(1+3 \delta_{3}\right) & \bar{x}_{4}\left(1-1.5 \delta_{4}\right) \\
\bar{x}_{1}\left(1+3 \delta_{1}\right) & \bar{x}_{2} & \bar{x}_{3}\left(1+3 \delta_{3}\right) & \bar{x}_{4}\left(1-1.5 \delta_{4}\right)
\end{array}\right]
$$

Os coeficientes propostos para problemas com 5 variáveis aleatórias são: 


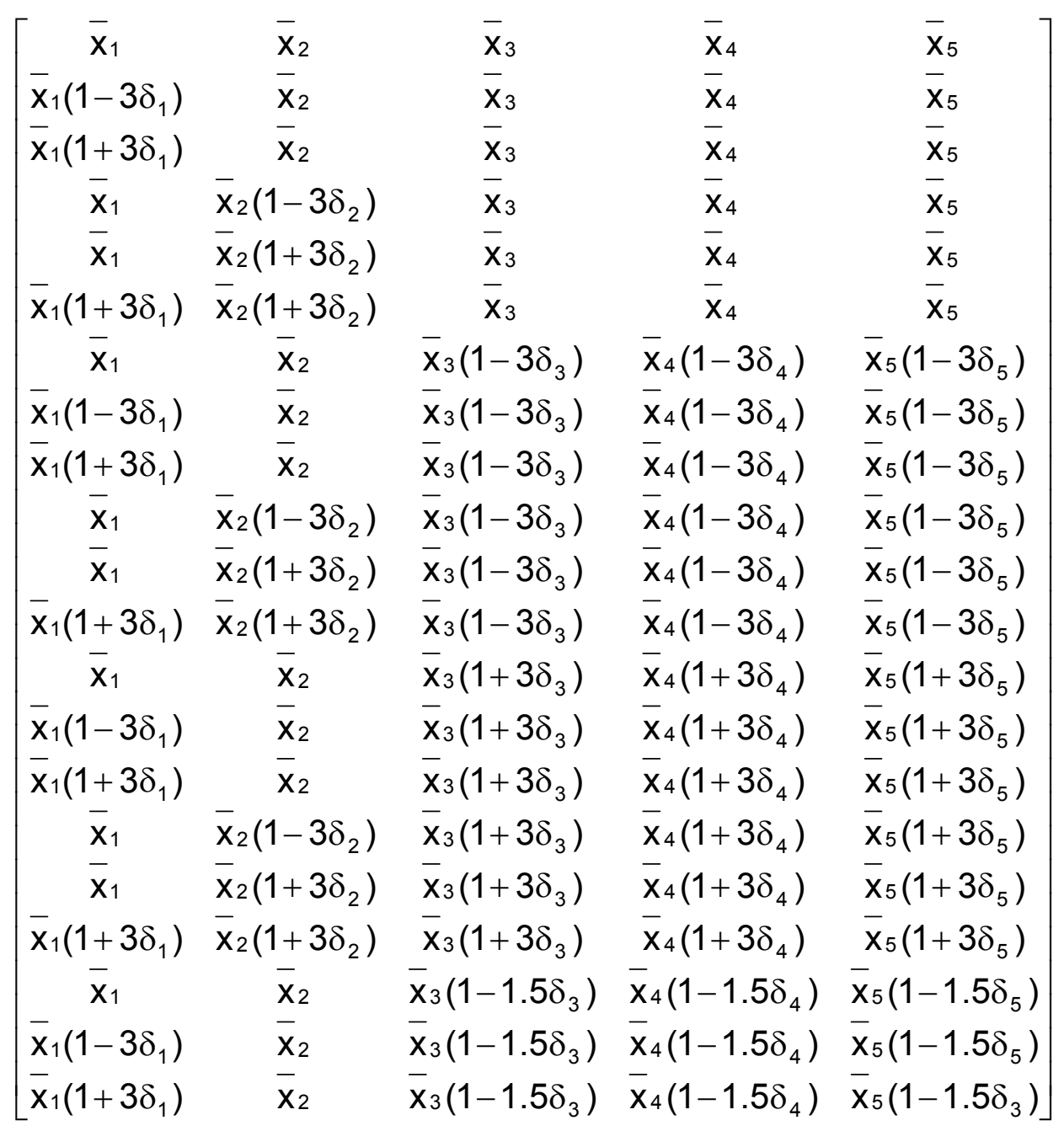

Os coeficientes propostos para problemas com 6 variáveis aleatórias são: 


\begin{tabular}{|c|c|c|c|c|c|}
\hline$\overline{\mathrm{X}}_{1}$ & $\bar{x}_{2}$ & $\bar{x}_{3}$ & $\bar{X}_{4}$ & $\bar{X}_{5}$ & $\bar{X}_{6}$ \\
\hline $\bar{x}_{1}\left(1-3 \delta_{1}\right)$ & $X_{2}$ & $\bar{x}_{3}\left(1-3 \delta_{3}\right)$ & $\overline{\mathrm{X}}_{4}\left(1-3 \delta_{4}\right)$ & $\bar{x}_{5}\left(1-3 \delta_{5}\right)$ & $\bar{x}_{6}\left(1-3 \delta_{6}\right)$ \\
\hline$\overline{\mathrm{x}}_{1}\left(1+3 \delta_{1}\right)$ & $\bar{x}_{2}$ & $\overline{\mathrm{x}}_{3}\left(1-3 \delta_{3}\right)$ & $\overline{\mathrm{x}}_{4}\left(1-3 \delta_{4}\right)$ & $\overline{\mathrm{x}}_{5}\left(1-3 \delta_{5}\right)$ & $\overline{\mathrm{x}}_{6}\left(1-3 \delta_{6}\right)$ \\
\hline $\bar{x}_{1}$ & $\bar{x}_{2}\left(1-3 \delta_{2}\right)$ & $\overline{\mathrm{x}}_{3}\left(1-3 \delta_{3}\right)$ & $\overline{\mathrm{x}}_{4}\left(1-3 \delta_{4}\right)$ & $\bar{x}_{5}\left(1-3 \delta_{5}\right)$ & $\bar{x}_{6}\left(1-3 \delta_{6}\right)$ \\
\hline$X_{1}$ & $\bar{x}_{2}\left(1+3 \delta_{2}\right)$ & $\bar{x}_{3}\left(1-3 \delta_{3}\right)$ & $\bar{x}_{4}\left(1-3 \delta_{4}\right)$ & $\bar{x}_{5}\left(1-3 \delta_{5}\right)$ & $\bar{x}_{6}\left(1-3 \delta_{6}\right)$ \\
\hline $\bar{x}_{1}\left(1+3 \delta_{1}\right)$ & $\overline{\mathrm{x}}_{2}\left(1+3 \delta_{2}\right)$ & $\bar{x}_{3}\left(1-3 \delta_{3}\right)$ & $\bar{x}_{4}\left(1-3 \delta_{4}\right)$ & $\bar{x}_{5}\left(1-3 \delta_{5}\right)$ & $\bar{x}_{6}\left(1-3 \delta_{6}\right)$ \\
\hline $\bar{x}_{1}$ & $\bar{x}_{2}$ & $\bar{x}_{3}\left(1-3 \delta_{3}\right)$ & $\bar{x}_{4}\left(1-3 \delta_{4}\right)$ & $\bar{x}_{5}\left(1-3 \delta_{5}\right)$ & $\bar{x}_{6}\left(1-3 \delta_{6}\right)$ \\
\hline$\overline{\mathrm{x}}_{1}\left(1-3 \delta_{1}\right)$ & $\mathrm{X}_{2}$ & $\overline{\mathrm{x}}_{3}\left(1+3 \delta_{3}\right)$ & $\overline{\mathrm{X}}_{4}\left(1+3 \delta_{4}\right)$ & $\overline{\mathrm{X}}_{5}\left(1+3 \delta_{5}\right)$ & $\overline{\mathrm{x}}_{6}\left(1+3 \delta_{6}\right)$ \\
\hline $\bar{x}_{1}\left(1+3 \delta_{1}\right)$ & $\mathrm{X}_{2}$ & $\bar{x}_{3}\left(1+3 \delta_{3}\right)$ & $\bar{x}_{4}\left(1+3 \delta_{4}\right)$ & $\bar{x}_{5}\left(1+3 \delta_{5}\right)$ & $\bar{x}_{6}\left(1+3 \delta_{6}\right)$ \\
\hline $\bar{x}_{1}$ & $\bar{x}_{2}\left(1-3 \delta_{2}\right)$ & $\bar{x}_{3}\left(1+3 \delta_{3}\right)$ & $\bar{x}_{4}\left(1+3 \delta_{4}\right.$ & $\bar{x}_{5}\left(1+3 \delta_{5}\right)$ & $\bar{x}_{6}\left(1+3 \delta_{6}\right)$ \\
\hline$\overline{\mathrm{X}}_{1}$ & $\overline{\mathrm{x}}_{2}\left(1+3 \delta_{2}\right)$ & $\bar{x}_{3}\left(1+3 \delta_{3}\right)$ & $\bar{x}_{4}\left(1+3 \delta_{4}\right)$ & $\bar{x}_{5}\left(1+3 \delta_{5}\right)$ & $\bar{x}_{6}\left(1+3 \delta_{6}\right)$ \\
\hline$\overline{\mathrm{x}}_{1}\left(1+3 \delta_{1}\right)$ & $\bar{x}_{2}\left(\underset{-}{1+3 \delta_{2}}\right)$ & $\bar{x}_{3}\left(1+3 \delta_{3}\right)$ & $\bar{x}_{4}\left(1+3 \delta_{4}\right)$ & $\bar{x}_{5}\left(1+3 \delta_{5}\right)$ & $\bar{x}_{6}\left(1+3 \delta_{6}\right)$ \\
\hline $\bar{x}_{1}$ & $\bar{x}_{2}$ & $\bar{x}_{3}\left(1+3 \delta_{3}\right)$ & $\bar{x}_{4}\left(1+3 \delta_{4}\right)$ & $\bar{x}_{5}\left(1+3 \delta_{5}\right)$ & $\bar{x}_{6}\left(1+3 \delta_{6}\right)$ \\
\hline $\bar{x}_{1}\left(1-3 \delta_{1}\right)$ & $X_{2}$ & $\bar{x}_{3}\left(1-1.5 \delta_{3}\right)$ & $\overline{\mathrm{x}}_{4}\left(1-1.5 \delta_{4}\right)$ & $\bar{x}_{5}\left(1-1.5 \delta_{5}\right)$ & $\bar{x}_{6}\left(1-1.5 \delta_{6}\right)$ \\
\hline $\bar{x}_{1}\left(1+3 \delta_{1}\right)$ & $\bar{x}_{2}$ & $\bar{x}_{3}\left(1-1.5 \delta_{3}\right)$ & $\bar{x}_{4}\left(1-1.5 \delta_{4}\right)$ & $\bar{x}_{5}\left(1-1.5 \delta_{5}\right)$ & $\bar{x}_{6}\left(1-1.5 \delta_{6}\right)$ \\
\hline $\bar{x}_{1}$ & $\bar{x}_{2}\left(1-3 \delta_{2}\right)$ & $\bar{x}_{3}\left(1-1.5 \delta_{3}\right)$ & $\bar{x}_{4}\left(1-1.5 \delta_{4}\right)$ & $\bar{x}_{5}\left(1-1.5 \delta_{5}\right)$ & $\bar{x}_{6}\left(1-1.5 \delta_{6}\right)$ \\
\hline $\bar{x}_{1}$ & $\overline{\mathrm{x}}_{2}\left(1+3 \delta_{2}\right)$ & $\bar{x}_{3}\left(1-1.5 \delta_{3}\right)$ & $\overline{\mathrm{x}}_{4}\left(1-1.5 \delta_{4}\right)$ & $\bar{x}_{5}\left(1-1.5 \delta_{5}\right)$ & $\bar{x}_{6}\left(1-1.5 \delta_{6}\right)$ \\
\hline $\bar{x}_{1}\left(1+3 \delta_{1}\right)$ & $\bar{x}_{2}\left(1+3 \delta_{2}\right)$ & $\bar{x}_{3}\left(1-1.5 \delta_{3}\right)$ & $\bar{x}_{4}\left(1-1.5 \delta_{4}\right)$ & $\bar{x}_{5}\left(1-1.5 \delta_{5}\right)$ & $\bar{x}_{6}\left(1-1.5 \delta_{6}\right)$ \\
\hline $\bar{x}_{1}$ & $\bar{x}_{2}$ & $\bar{x}_{3}\left(1-1.5 \delta_{3}\right)$ & $\bar{x}_{4}\left(1-1.5 \delta_{4}\right)$ & $\bar{x}_{5}\left(1-1.5 \delta_{5}\right)$ & $\bar{x}_{6}\left(1-1.5 \delta_{6}\right)$ \\
\hline $\bar{x}_{1}\left(1-3 \delta_{1}\right)$ & $\bar{x}_{2}$ & $\bar{x}_{3}\left(1+1.5 \delta_{3}\right)$ & $\bar{x}_{4}\left(1+1.5 \delta_{4}\right)$ & $\bar{x}_{5}\left(1+1.5 \delta_{5}\right)$ & $\bar{x}_{6}\left(1+1.5 \delta_{6}\right)$ \\
\hline$\overline{\mathrm{x}}_{1}\left(1+3 \delta_{1}\right)$ & $\mathrm{X}_{2}$ & $\bar{x}_{3}\left(1+1.5 \delta_{3}\right)$ & $\overline{\mathrm{x}}_{4}\left(1+1.5 \delta_{4}\right)$ & $\bar{x}_{5}\left(1+1.5 \delta_{5}\right)$ & $\bar{x}_{6}\left(1+1.5 \delta_{6}\right)$ \\
\hline $\bar{x}_{1}$ & $\bar{x}_{2}\left(1-3 \delta_{2}\right)$ & $\bar{x}_{3}\left(1+1.5 \delta_{3}\right)$ & $\bar{x}_{4}\left(1+1.5 \delta_{4}\right)$ & $\bar{x}_{5}\left(1+1.5 \delta_{5}\right)$ & $\bar{x}_{6}\left(1+1.5 \delta_{6}\right)$ \\
\hline $\mathrm{X}_{1}$ & $\bar{x}_{2}\left(1+3 \delta_{2}\right)$ & $\bar{x}_{3}\left(1+1.5 \delta_{3}\right)$ & $\bar{x}_{4}\left(1+1.5 \delta_{4}\right)$ & $\bar{x}_{5}\left(1+1.5 \delta_{5}\right)$ & $\bar{x}_{6}\left(1+1.5 \delta_{6}\right)$ \\
\hline$\overline{\mathrm{x}}_{1}\left(1+3 \delta_{1}\right)$ & $\bar{x}_{2}\left(1+3 \delta_{2}\right)$ & $\bar{x}_{3}\left(1+1.5 \delta_{3}\right)$ & $\bar{x}_{4}\left(1+1.5 \delta_{4}\right)$ & $\overline{\mathrm{X}}_{5}\left(1+1.5 \delta_{5}\right)$ & $\bar{x}_{6}\left(1+1.5 \delta_{6}\right)$ \\
\hline$-\mathrm{X}_{1}$ & $\overline{\mathrm{x}}_{2}$ & $\bar{x}_{3}\left(1+1.5 \delta_{3}\right)$ & $\bar{x}_{4}\left(1+1.5 \delta_{4}\right)$ & $\bar{x}_{5}\left(1+1.5 \delta_{5}\right)$ & $\bar{x}_{6}\left(1+1.5 \delta_{6}\right)$ \\
\hline $\bar{x}_{1}\left(1-3 \delta_{1}\right)$ & $\bar{x}_{2}$ & $\bar{x}_{3}$ & $\bar{x}_{4}\left(1-3 \delta_{4}\right)$ & $\bar{x}_{5}\left(1+3 \delta_{5}\right)$ & $\bar{x}_{6}\left(1+3 \delta_{6}\right)$ \\
\hline $\begin{array}{c}\bar{x}_{1}\left(1+3 \delta_{1}\right) \\
-\end{array}$ & $-\bar{x}_{2}$ & $\bar{x}_{3}$ & $\bar{x}_{4}\left(1-3 \delta_{4}\right)$ & $\bar{x}_{5}\left(1+3 \delta_{5}\right)$ & $\bar{x}_{6}\left(1+3 \delta_{6}\right)$ \\
\hline $\bar{X}_{1}$ & $\bar{x}_{2}\left(1-3 \delta_{2}\right)$ & $\bar{x}_{3}$ & $\mathrm{x}_{4}\left(1-3 \delta_{4}\right)$ & $\mathrm{x}_{5}\left(1+3 \delta_{5}\right)$ & $\mathrm{x}_{6}\left(1+3 \delta_{6}\right)$ \\
\hline
\end{tabular}

\subsubsection{Plano de Experiência Numérico Composto}

Esse plano apresenta uma ótima performance, embora aparentemente tenha pontos em exagero. Para estruturas com elevado grau de nãolinearidade, parece ser o mais adequado dentre os planos apresentados.

Os coeficientes propostos para problemas com 1 variável aleatória são: 


$$
\left\{\begin{array}{lllll}
\bar{x}_{1} & \bar{x}_{1}\left(1-3 \delta_{1}\right) & \bar{x}_{1}\left(1-1.5 \delta_{1}\right) & \bar{x}_{1}\left(1+1.5 \delta_{1}\right) & \bar{x}_{1}\left(1+3 \delta_{1}\right)
\end{array}\right\}
$$

Os coeficientes propostos para problemas com 2 variáveis aleatórias são:

$$
\left[\begin{array}{cc}
\overline{\mathrm{x}}_{1} & \overline{\mathrm{x}}_{2}\left(1+3 \delta_{2}\right) \\
\overline{\mathrm{x}}_{1}\left(1-1.5 \delta_{1}\right) & \overline{\mathrm{x}}_{2}\left(1+1.5 \delta_{2}\right) \\
\overline{\mathrm{x}}_{1}\left(1+1.5 \delta_{1}\right) & \overline{\mathrm{x}}_{2}\left(1+1.5 \delta_{2}\right) \\
\overline{\mathrm{x}}_{1}\left(1-3 \delta_{1}\right) & \overline{\mathrm{x}}_{2} \\
\overline{\mathrm{x}}_{1}\left(1+3 \delta_{1}\right) & \overline{\mathrm{x}}_{2} \\
\overline{\mathrm{x}}_{1}\left(1-1.5 \delta_{1}\right) & \overline{\mathrm{x}}_{2}\left(1-1.5 \delta_{2}\right) \\
\overline{\mathrm{x}}_{1}\left(1+1.5 \delta_{1}\right) & \overline{\mathrm{x}}_{2}\left(1-1.5 \delta_{2}\right) \\
\overline{\mathrm{x}}_{1} & \overline{\mathrm{x}}_{2}\left(1-3 \delta_{2}\right) \\
\overline{\mathrm{x}}_{1} & \overline{\mathrm{x}}_{2}
\end{array}\right]
$$

Os coeficientes propostos para problemas com 3 variáveis aleatórias são:

$$
\left[\begin{array}{ccc}
\overline{\mathrm{x}}_{1} & \overline{\mathrm{x}}_{2}\left(1+3 \delta_{2}\right) & \overline{\mathrm{x}}_{3} \\
\overline{\mathrm{x}}_{1}\left(1-1.5 \delta_{1}\right) & \overline{\mathrm{x}}_{2}\left(1+1.5 \delta_{2}\right) & \overline{\mathrm{x}}_{3} \\
\overline{\mathrm{x}}_{1}\left(1+1.5 \delta_{1}\right) & \overline{\mathrm{x}}_{2}\left(1+1.5 \delta_{2}\right) & \overline{\mathrm{x}}_{3} \\
\overline{\mathrm{x}}_{1}\left(1-3 \delta_{1}\right) & \overline{\mathrm{x}}_{2} & \overline{\mathrm{x}}_{3} \\
\overline{\mathrm{x}}_{1}\left(1+3 \delta_{1}\right) & \overline{\mathrm{x}}_{2} & \overline{\mathrm{x}}_{3} \\
\overline{\mathrm{x}}_{1}\left(1-1.5 \delta_{1}\right) & \overline{\mathrm{x}}_{2}\left(1-1.5 \delta_{2}\right) & \overline{\mathrm{x}}_{3} \\
\overline{\mathrm{x}}_{1}\left(1+1.5 \delta_{1}\right) & \overline{\mathrm{x}}_{2}\left(1-1.5 \delta_{2}\right) & \overline{\mathrm{x}}_{3} \\
\overline{\mathrm{x}}_{1} & \overline{\mathrm{x}}_{2}\left(1-3 \delta_{2}\right) & \overline{\mathrm{x}}_{3} \\
\overline{\mathrm{x}}_{1} & \overline{\mathrm{x}}_{2} & \overline{\mathrm{x}}_{3} \\
\overline{\mathrm{x}}_{1} & \overline{\mathrm{x}}_{2}\left(1+3 \delta_{2}\right) & \overline{\mathrm{x}}_{3}\left(1-3 \delta_{3}\right) \\
\overline{\mathrm{x}}_{1}\left(1-1.5 \delta_{1}\right) & \overline{\mathrm{x}}_{2}\left(1+1.5 \delta_{2}\right) & \overline{\mathrm{x}}_{3}\left(1-3 \delta_{3}\right) \\
\overline{\mathrm{x}}_{1}\left(1+1.5 \delta_{1}\right) & \overline{\mathrm{x}}_{2}\left(1+1.5 \delta_{2}\right) & \overline{\mathrm{x}}_{3}\left(1-3 \delta_{3}\right) \\
\overline{\mathrm{x}}_{1}\left(1-3 \delta_{1}\right) & \overline{\mathrm{x}}_{2} & \overline{\mathrm{x}}_{3}\left(1-3 \delta_{3}\right) \\
\overline{\mathrm{x}}_{1}\left(1+3 \delta_{1}\right) & \overline{\mathrm{x}}_{2} & \overline{\mathrm{x}}_{3}\left(1-3 \delta_{3}\right) \\
\overline{\mathrm{x}}_{1}\left(1-1.5 \delta_{1}\right) & \overline{\mathrm{x}}_{2}\left(1-1.5 \delta_{2}\right) & \overline{\mathrm{x}}_{3}\left(1+3 \delta_{3}\right) \\
\overline{\mathrm{x}}_{1}\left(1+1.5 \delta_{1}\right) & \overline{\mathrm{x}}_{2}\left(1-1.5 \delta_{2}\right) & \overline{\mathrm{x}}_{3}\left(1+3 \delta_{3}\right) \\
\overline{\mathrm{x}}_{1} & \overline{\mathrm{x}}_{2}\left(1-3 \delta_{2}\right) & \overline{\mathrm{x}}_{3}\left(1+3 \delta_{3}\right) \\
\overline{\mathrm{x}}_{1} & \overline{\mathrm{x}}_{2} & \overline{\mathrm{x}}_{3}\left(1+3 \delta_{3}\right)
\end{array}\right]
$$


Os coeficientes propostos para problemas com 4 variáveis aleatórias são:

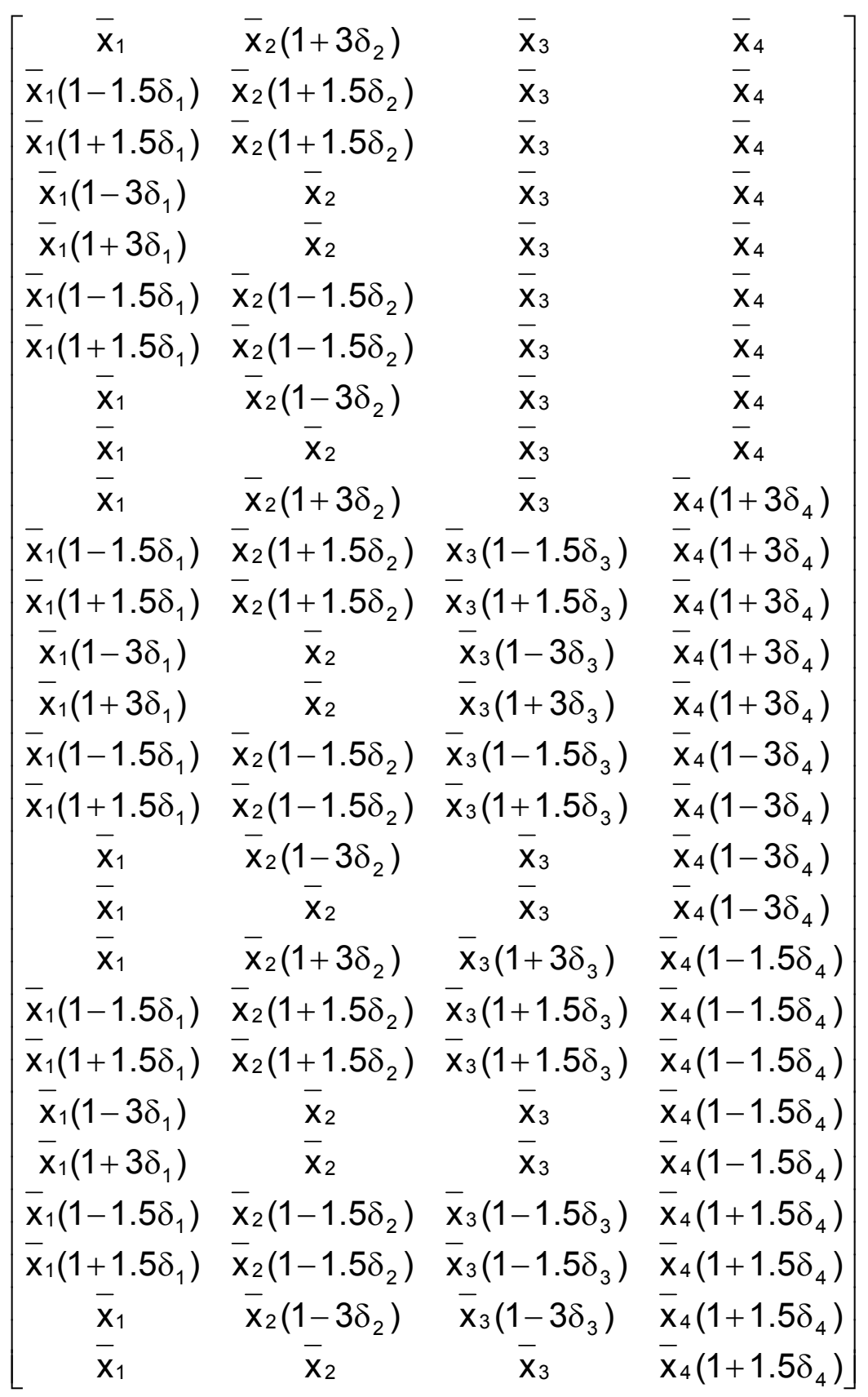

Os coeficientes propostos para problemas com 5 variáveis aleatórias são: 


$\left[\begin{array}{ccccc}\overline{\mathrm{x}}_{1} & \overline{\mathrm{x}}_{2}\left(1+3 \delta_{2}\right) & \overline{\mathrm{x}}_{3} & \overline{\mathrm{x}}_{4} & \overline{\mathrm{x}}_{5} \\ \overline{\mathrm{x}}_{1}\left(1-1.5 \delta_{1}\right) & \overline{\mathrm{x}}_{2}\left(1+1.5 \delta_{2}\right) & \overline{\mathrm{x}}_{3} & \overline{\mathrm{x}}_{4} & \overline{\mathrm{x}}_{5} \\ \overline{\mathrm{x}}_{1}\left(1+1.5 \delta_{1}\right) & \overline{\mathrm{x}}_{2}\left(1+1.5 \delta_{2}\right) & \overline{\mathrm{x}}_{3} & \overline{\mathrm{x}}_{4} & \overline{\mathrm{x}}_{5} \\ \overline{\mathrm{x}}_{1}\left(1-3 \delta_{1}\right) & \overline{\mathrm{x}}_{2} & \overline{\mathrm{x}}_{3} & \overline{\mathrm{x}}_{4} & \overline{\mathrm{x}}_{5} \\ \overline{\mathrm{x}}_{1}\left(1+3 \delta_{1}\right) & \overline{\mathrm{x}}_{2} & \overline{\mathrm{x}}_{3} & \overline{\mathrm{x}}_{4} & \overline{\mathrm{x}}_{5} \\ \overline{\mathrm{x}}_{1}\left(1-1.5 \delta_{1}\right) & \overline{\mathrm{x}}_{2}\left(1-1.5 \delta_{2}\right) & \overline{\mathrm{x}}_{3} & \overline{\mathrm{x}}_{4} & \overline{\mathrm{x}}_{5} \\ \overline{\mathrm{x}}_{1}\left(1+1.5 \delta_{1}\right) & \overline{\mathrm{x}}_{2}\left(1-1.5 \delta_{2}\right) & \overline{\mathrm{x}}_{3} & \overline{\mathrm{x}}_{4} & \overline{\mathrm{x}}_{5} \\ \overline{\mathrm{x}}_{1} & \overline{\mathrm{x}}_{2}\left(1-3 \delta_{2}\right) & \overline{\mathrm{x}}_{3} & \overline{\mathrm{x}}_{4} & \overline{\mathrm{x}}_{5} \\ \overline{\mathrm{x}}_{1} & \overline{\mathrm{x}}_{2} & \overline{\mathrm{x}}_{3} & \overline{\mathrm{x}}_{4} & \overline{\mathrm{x}}_{5} \\ \overline{\mathrm{x}}_{1} & \overline{\mathrm{x}}_{2}\left(1+3 \delta_{2}\right) & \overline{\mathrm{x}}_{3} & \overline{\mathrm{x}}_{4}\left(1+3 \delta_{4}\right) & \overline{\mathrm{x}}_{5}\left(1+3 \delta_{5}\right) \\ \overline{\mathrm{x}}_{1}\left(1-1.5 \delta_{1}\right) & \overline{\mathrm{x}}_{2}\left(1+1.5 \delta_{2}\right) & \overline{\mathrm{x}}_{3}\left(1-1.5 \delta_{3}\right) & \overline{\mathrm{x}}_{4}\left(1+3 \delta_{4}\right) & \overline{\mathrm{x}}_{5}\left(1+3 \delta_{5}\right) \\ \overline{\mathrm{x}}_{1}\left(1+1.5 \delta_{1}\right) & \overline{\mathrm{x}}_{2}\left(1+1.5 \delta_{2}\right) & \overline{\mathrm{x}}_{3}\left(1+1.5 \delta_{3}\right) & \overline{\mathrm{x}}_{4}\left(1+3 \delta_{4}\right) & \overline{\mathrm{x}}_{5}\left(1+3 \delta_{5}\right) \\ \overline{\mathrm{x}}_{1}\left(1-3 \delta_{1}\right) & \overline{\mathrm{x}}_{2} & \overline{\mathrm{x}}_{3}\left(1-3 \delta_{3}\right) & \overline{\mathrm{x}}_{4}\left(1+3 \delta_{4}\right) & \overline{\mathrm{x}}_{5}\left(1+3 \delta_{5}\right) \\ \overline{\mathrm{x}}_{1}\left(1+3 \delta_{1}\right) & \overline{\mathrm{x}}_{2} & \overline{\mathrm{x}}_{3}\left(1+3 \delta_{3}\right) & \overline{\mathrm{x}}_{4}\left(1+3 \delta_{4}\right) & \overline{\mathrm{x}}_{5}\left(1+3 \delta_{5}\right) \\ \overline{\mathrm{x}}_{1}\left(1-1.5 \delta_{1}\right) & \overline{\mathrm{x}}_{2}\left(1-1.5 \delta_{2}\right) & \overline{\mathrm{x}}_{3}\left(1-1.5 \delta_{3}\right) & \overline{\mathrm{x}}_{4}\left(1+3 \delta_{4}\right) & \overline{\mathrm{x}}_{5}\left(1+3 \delta_{5}\right) \\ \overline{\mathrm{x}}_{1}\left(1+1.5 \delta_{1}\right) & \overline{\mathrm{x}}_{2}\left(1-1.5 \delta_{2}\right) & \overline{\mathrm{x}}_{3}\left(1+1.5 \delta_{3}\right) & \overline{\mathrm{x}}_{4}\left(1+3 \delta_{4}\right) & \overline{\mathrm{x}}_{5}\left(1+3 \delta_{5}\right) \\ \overline{\mathrm{x}}_{1} & \overline{\mathrm{x}}_{2}\left(1-3 \delta_{2}\right) & \overline{\mathrm{x}}_{3} & \overline{\mathrm{x}}_{4}\left(1+3 \delta_{4}\right) & \overline{\mathrm{x}}_{5}\left(1+3 \delta_{5}\right) \\ \overline{\mathrm{x}}_{1} & \overline{\mathrm{x}}_{2} & \overline{\mathrm{x}}_{3} & \overline{\mathrm{x}}_{4}\left(1+3 \delta_{4}\right) & \overline{\mathrm{x}}_{5}\left(1+3 \delta_{5}\right)\end{array}\right]$

\begin{tabular}{|c|c|c|c|c|}
\hline $\mathrm{X}_{1}$ & $\bar{x}_{2}\left(1+3 \delta_{2}\right)$ & $\bar{x}_{3}\left(1+3 \delta_{3}\right)$ & $\mathrm{x}_{4}\left(1-3 \delta_{4}\right)$ & $\bar{x}_{5}\left(1-3 \delta_{5}\right)$ \\
\hline$\overline{\mathrm{x}}_{1}\left(1-1.5 \delta_{1}\right)$ & $\bar{x}_{2}\left(1+1.5 \delta_{2}\right)$ & $\bar{x}_{3}\left(1+1.5 \delta_{3}\right)$ & $\overline{\mathrm{X}}_{4}\left(1-3 \delta_{4}\right)$ & $\overline{\mathrm{X}}_{5}\left(1-3 \delta_{5}\right)$ \\
\hline$\overline{\mathrm{x}}_{1}\left(1+1.5 \delta_{1}\right)$ & $\bar{x}_{2}\left(1+1.5 \delta_{2}\right)$ & $\bar{x}_{3}\left(1+1.5 \delta_{3}\right)$ & $\overline{\mathrm{x}}_{4}\left(1-3 \delta_{4}\right)$ & $\bar{x}_{5}\left(1-3 \delta_{5}\right)$ \\
\hline $\bar{x}_{1}\left(1-3 \delta_{1}\right)$ & $\bar{x}_{2}$ & $\bar{x}_{3}$ & $\overline{\mathrm{x}}_{4}\left(1-3 \delta_{4}\right)$ & $\bar{x}_{5}\left(1-3 \delta_{5}\right)$ \\
\hline $\bar{x}_{1}\left(1+3 \delta_{1}\right)$ & $-\bar{x}_{2}$ & $-\bar{x}_{3}$ & $\bar{x}_{4}\left(1-3 \delta_{4}\right)$ & $\bar{x}_{5}\left(1-3 \delta_{5}\right)$ \\
\hline$\overline{\mathrm{x}}_{1}\left(1-1.5 \delta_{1}\right)$ & $\bar{x}_{2}\left(1-1.5 \delta_{2}\right)$ & $\bar{x}_{3}\left(1-1.5 \delta_{3}\right)$ & $\bar{x}_{4}\left(1-3 \delta_{4}\right)$ & $\bar{x}_{5}\left(1-3 \delta_{5}\right)$ \\
\hline$\overline{\mathrm{X}}_{1}\left(1+1.5 \delta_{1}\right)$ & $\overline{\mathrm{X}}_{2}\left(1-1.5 \delta_{2}\right)$ & $\overline{\mathrm{X}}_{3}\left(1-1.5 \delta_{3}\right)$ & $\overline{\mathrm{x}}_{4}\left(1-3 \delta_{4}\right)$ & $\bar{x}_{5}\left(1-3 \delta_{5}\right)$ \\
\hline $\bar{x}_{1}$ & $\bar{x}_{2}\left(1-3 \delta_{2}\right)$ & $\bar{x}_{3}\left(1-3 \delta_{3}\right)$ & $\mathrm{x}_{4}\left(1-3 \delta_{4}\right)$ & $\mathrm{x}_{5}\left(1-3 \delta_{5}\right)$ \\
\hline$\overline{\mathbf{X}}_{1}$ & $\bar{x}_{2}$ & $\bar{x}_{3}$ & $\bar{x}_{4}\left(1-3 \delta_{4}\right)$ & $\bar{x}_{5}\left(1-3 \delta_{5}\right)$ \\
\hline$\overline{\mathrm{X}}_{1}$ & $\bar{x}_{2}\left(1+3 \delta_{2}\right)$ & $\bar{x}_{3}\left(1+2 \delta_{3}\right)$ & $\bar{x}_{4}\left(1+1.5 \delta_{4}\right)$ & $\bar{x}_{5}\left(1-1.5 \delta_{5}\right)$ \\
\hline$\overline{\mathrm{x}}_{1}\left(1-1.5 \delta_{1}\right)$ & $\overline{\mathrm{x}}_{2}\left(1+1.5 \delta_{2}\right)$ & $\bar{x}_{3}\left(1+2 \delta_{3}\right)$ & $\bar{x}_{4}\left(1+1.5 \delta_{4}\right)$ & $\bar{x}_{5}\left(1-1.5 \delta_{5}\right)$ \\
\hline$\overline{\mathrm{x}}_{1}\left(1+1.5 \delta_{1}\right)$ & $\bar{x}_{2}\left(1+1.5 \delta_{2}\right)$ & $\overline{\mathrm{X}}_{3}\left(1+2 \delta_{3}\right)$ & $\bar{x}_{4}\left(1+1.5 \delta_{4}\right)$ & $\bar{x}_{5}\left(1-1.5 \delta_{5}\right)$ \\
\hline $\bar{x}_{1}\left(1-3 \delta_{1}\right)$ & $\bar{x}_{2}$ & $\bar{x}_{3}\left(1+2 \delta_{3}\right)$ & $\bar{x}_{4}\left(1+1.5 \delta_{4}\right)$ & $\bar{x}_{5}\left(1-1.5 \delta_{5}\right)$ \\
\hline$\overline{\mathrm{x}}_{1}\left(1+3 \delta_{1}\right)$ & $\bar{x}_{2}$ & $\bar{x}_{3}\left(1+2 \delta_{3}\right)$ & $\bar{x}_{4}\left(1+1.5 \delta_{4}\right)$ & $\bar{x}_{5}\left(1-1.5 \delta_{5}\right)$ \\
\hline$\overline{\mathrm{x}}_{1}\left(1-1.5 \delta_{1}\right)$ & $\bar{x}_{2}\left(1-1.5 \delta_{2}\right)$ & $\bar{x}_{3}\left(1+2 \delta_{3}\right)$ & $\bar{x}_{4}\left(1+1.5 \delta_{4}\right)$ & $\bar{x}_{5}\left(1-1.5 \delta_{5}\right)$ \\
\hline$\overline{\mathrm{x}}_{1}\left(1+1.5 \delta_{1}\right)$ & $\bar{x}_{2}\left(1-1.5 \delta_{2}\right)$ & $\bar{x}_{3}\left(1+2 \delta_{3}\right)$ & $\bar{x}_{4}\left(1+1.5 \delta_{4}\right)$ & $\bar{x}_{5}\left(1-1.5 \delta_{5}\right)$ \\
\hline $\bar{x}_{1}$ & $\overline{\mathrm{x}}_{2}\left(1-3 \delta_{2}\right)$ & $\bar{x}_{3}\left(1+2 \delta_{3}\right)$ & $\overline{\mathrm{X}}_{4}\left(1+1.5 \delta_{4}\right)$ & $\overline{\mathrm{X}}_{5}\left(1-1.5 \delta_{5}\right)$ \\
\hline$\overline{\mathbf{X}}_{1}$ & $\overline{\mathrm{x}}_{2}$ & $\overline{\mathrm{x}}_{3}\left(1+2 \delta_{3}\right)$ & $\overline{\mathrm{X}}_{4}\left(1+1.5 \delta_{4}\right)$ & $\bar{x}_{5}\left(1-1.5 \delta_{5}\right)$ \\
\hline
\end{tabular}


Os coeficientes propostos para problemas com 6 variáveis aleatórias são:

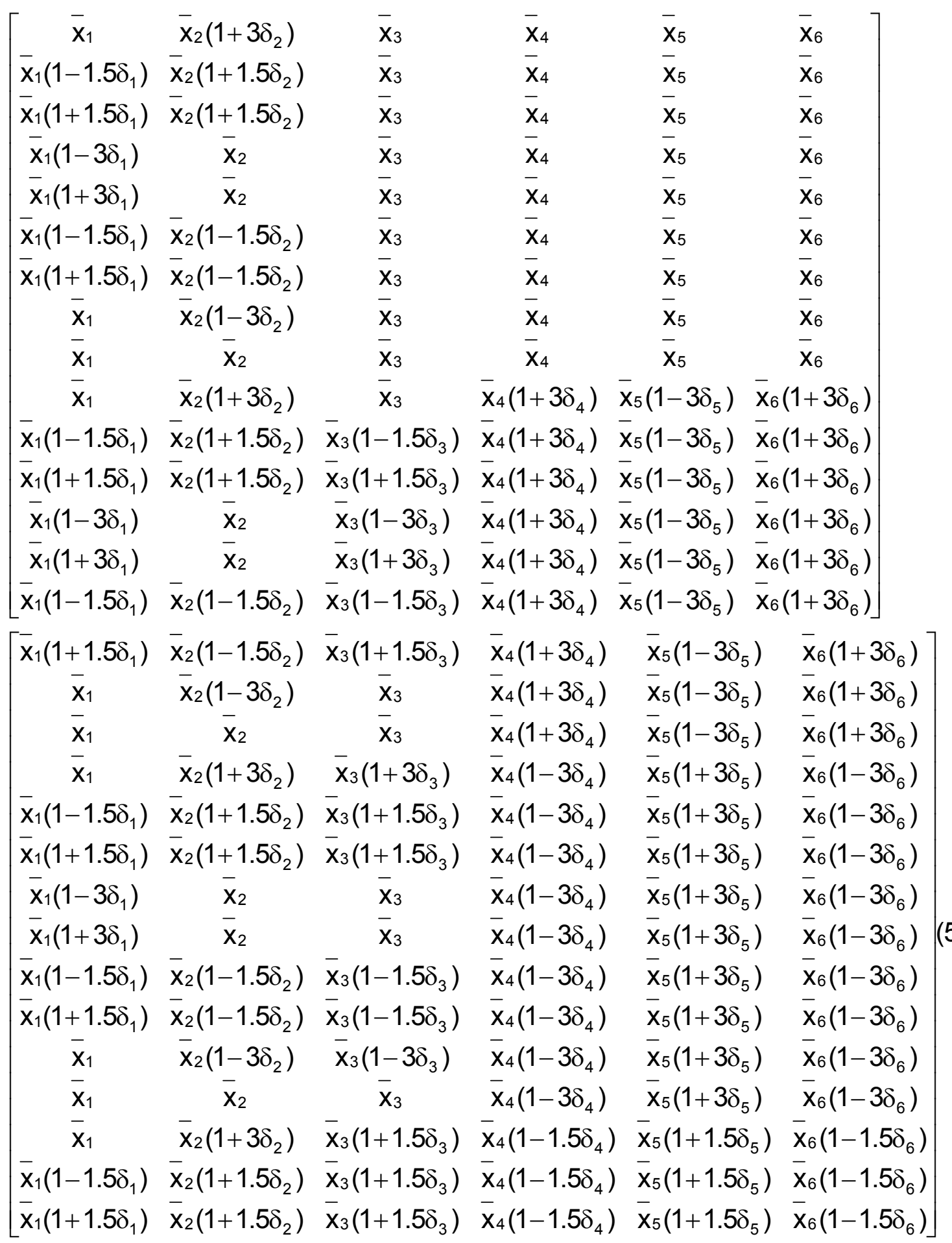




\begin{tabular}{|c|c|c|c|c|c|}
\hline $\bar{x}_{1}\left(1-3 \delta_{1}\right)$ & $\bar{x}_{2}$ & $\bar{x}_{3}\left(1+1.5 \delta_{3}\right)$ & $\overline{\mathrm{X}}_{4}\left(1-1.5 \delta_{4}\right)$ & $\overline{\mathbf{X}}_{5}\left(1+1.5 \delta_{5}\right)$ & $\bar{x}_{6}\left(1-1.5 \delta_{6}\right)$ \\
\hline $\bar{x}_{1}\left(1+3 \delta_{1}\right)$ & $\bar{x}_{2}$ & $\overline{\mathrm{x}}_{3}\left(1+1.5 \delta_{3}\right)$ & $\overline{\mathrm{x}}_{4}\left(1-1.5 \delta_{4}\right)$ & $\bar{x}_{5}\left(1+1.5 \delta_{5}\right)$ & $\bar{x}_{6}\left(1-1.5 \delta_{6}\right)$ \\
\hline $\bar{x}_{1}\left(1-1.5 \delta_{1}\right)$ & $\bar{x}_{2}\left(1-1.5 \delta_{2}\right)$ & $\bar{x}_{3}\left(1+1.5 \delta_{3}\right)$ & $\bar{x}_{4}\left(1-1.5 \delta_{4}\right)$ & $\overline{\mathrm{X}}_{5}\left(1+1.5 \delta_{5}\right)$ & $\bar{x}_{6}\left(1-1.5 \delta_{6}\right)$ \\
\hline $\bar{x}_{1}\left(1+1.5 \delta_{1}\right)$ & $\overline{\mathrm{x}}_{2}\left(1-1.5 \delta_{2}\right)$ & $\bar{x}_{3}\left(1+1.5 \delta_{3}\right)$ & $\bar{x}_{4}\left(1-1.5 \delta_{4}\right)$ & $\overline{\mathrm{x}}_{5}\left(1+1.5 \delta_{5}\right)$ & $\bar{x}_{6}\left(1-1.5 \delta_{6}\right)$ \\
\hline $\bar{x}_{1}$ & $\bar{x}_{2}\left(1-3 \delta_{2}\right)$ & $\bar{x}_{3}\left(1+1.5 \delta_{3}\right)$ & $\overline{\mathrm{x}}_{4}\left(1-1.5 \delta_{4}\right)$ & $\bar{x}_{5}\left(1+1.5 \delta_{5}\right)$ & $\bar{x}_{6}\left(1-1.5 \delta_{6}\right)$ \\
\hline$\overline{\mathrm{x}}_{1}$ & $\bar{x}_{2}$ & $\bar{x}_{3}\left(1+1.5 \delta_{3}\right)$ & $\bar{x}_{4}\left(1-1.5 \delta_{4}\right)$ & $\overline{\mathrm{x}}_{5}\left(1+1.5 \delta_{5}\right)$ & $\bar{x}_{6}\left(1-1.5 \delta_{6}\right)$ \\
\hline$\overline{\mathbf{X}}_{1}$ & $\bar{x}_{2}\left(1+3 \delta_{2}\right)$ & $\bar{x}_{3}\left(1-1.5 \delta_{3}\right)$ & $\bar{x}_{4}\left(1+1.5 \delta_{4}\right)$ & $\bar{x}_{5}\left(1-1.5 \delta_{5}\right)$ & $\bar{x}_{6}\left(1+1.5 \delta_{6}\right)$ \\
\hline $\bar{x}_{1}\left(1-1.5 \delta_{1}\right)$ & $\bar{x}_{2}\left(1+1.5 \delta_{2}\right)$ & $\bar{x}_{3}\left(1-1.5 \delta_{3}\right)$ & $\bar{x}_{4}\left(1+1.5 \delta_{4}\right)$ & $\bar{x}_{5}\left(1-1.5 \delta_{5}\right)$ & $\bar{x}_{6}\left(1+1.5 \delta_{6}\right)$ \\
\hline $\bar{x}_{1}\left(1+1.5 \delta_{1}\right)$ & $\overline{\mathrm{x}}_{2}\left(1+1.5 \delta_{2}\right)$ & $\bar{x}_{3}\left(1-1.5 \delta_{3}\right)$ & $\bar{x}_{4}\left(1+1.5 \delta_{4}\right)$ & $\overline{\mathrm{x}}_{5}\left(1-1.5 \delta_{5}\right)$ & $\bar{x}_{6}\left(1+1.5 \delta_{6}\right)$ \\
\hline $\bar{x}_{1}\left(1-3 \delta_{1}\right)$ & $\bar{x}_{2}$ & $\overline{\mathrm{x}}_{3}\left(1-1.5 \delta_{3}\right)$ & $\bar{x}_{4}\left(1+1.5 \delta_{4}\right)$ & $\overline{\mathrm{X}}_{5}\left(1-1.5 \delta_{5}\right)$ & $\bar{x}_{6}\left(1+1.5 \delta_{6}\right)$ \\
\hline $\bar{x}_{1}\left(1+3 \delta_{1}\right)$ & $\bar{x}_{2}$ & $\bar{x}_{3}\left(1-1.5 \delta_{3}\right)$ & $\bar{x}_{4}\left(1+1.5 \delta_{4}\right)$ & $\overline{\mathrm{X}}_{5}\left(1-1.5 \delta_{5}\right)$ & $\left..5 \delta_{6}\right)$ \\
\hline $\bar{x}_{1}\left(1-1.5 \delta_{1}\right)$ & $\overline{\mathrm{x}}_{2}\left(1-1.5 \delta_{2}\right)$ & $\overline{\mathrm{x}}_{3}\left(1-1.5 \delta_{3}\right)$ & $\overline{\mathrm{x}}_{4}\left(1+1.5 \delta_{4}\right)$ & $\overline{\mathrm{X}}_{5}\left(1-1.5 \delta_{5}\right)$ & $\bar{x}_{6}\left(1+1.5 \delta_{6}\right)$ \\
\hline $\bar{x}_{1}\left(1+1.5 \delta_{1}\right)$ & $\overline{\mathrm{X}}_{2}\left(1-1.5 \delta_{2}\right)$ & $\overline{\mathrm{x}}_{3}\left(1-1.5 \delta_{3}\right)$ & $\overline{\mathrm{x}}_{4}\left(1+1.5 \delta_{4}\right)$ & $\overline{\mathrm{x}}_{5}\left(1-1.5 \delta_{5}\right)$ & $\bar{x}_{6}\left(1+1.5 \delta_{6}\right)$ \\
\hline $\mathbf{x}_{1}$ & $\mathrm{x}_{2}\left(1-3 \delta_{2}\right)$ & $\mathrm{x}_{3}\left(1-1.5 \delta_{3}\right)$ & $\mathrm{x}_{4}\left(1+1.5 \delta_{4}\right)$ & $\mathrm{x}_{5}\left(1-1.5 \delta_{5}\right)$ & $\mathrm{x}_{6}\left(1+1.5 \delta_{6}\right)$ \\
\hline$\overline{\mathrm{x}}_{1}$ & $\bar{x}_{2}$ & $\bar{x}_{3}\left(1-1.5 \delta_{3}\right)$ & $\overline{\mathrm{x}}_{4}\left(1+1.5 \delta_{4}\right)$ & $\overline{\mathrm{x}}_{5}\left(1-1.5 \delta_{5}\right)$ & $\bar{x}_{6}\left(1+1.5 \delta_{6}\right)$ \\
\hline
\end{tabular}

\subsubsection{Planos de Experiência Aleatórios}

O procedimento para os planos de experiência aleatórios é semelhante ao utilizado para gerar os planos de experiência numéricos, diferenciando-se apenas na geração dos pontos que nesse caso é aleatória.

É interessante não deixar que os coeficientes sejam completamente aleatórios. A aleatoriedade dos coeficientes deve pertencer a domínios bem definidos para evitar problemas com a solução mecânica da estrutura. É fácil entender essa observação, imagine se o coeficiente de resistência do material que compõe a estrutura for próximo de zero, nessa situação provavelmente a matriz de rigidez dessa estrutura será singular.

Um caminho bastante interessante para "direcionar" a aleatoriedade dos pontos desses planos é a utilização de algoritmos genéticos. A utilização de algoritmos heurísticos por natureza para gerar novos planos de experiência é a opção mais recomendada quando se trata de número elevado de variáveis aleatórias, ou número elevado de hipersuperfícies limites, ou problemas com elevado número de respostas ou mínimos locais. 


\section{ÍNDICE DE CONFIABILIDADE}

\subsection{Introdução}

$\mathrm{Na}$ maioria dos métodos utilizados para análise da confiabilidade necessita-se de um algoritmo de otimização para encontrar o ponto de projeto, ou a solução do problema. Nesses métodos, a otimização é utilizada para encontrar a mínima distância entre o ponto de projeto e o centro do sistema de coordenadas no espaço normal padrão não correlacionado.

Antes de comentar alguns algoritmos de otimização aplicados ao cálculo do índice de confiabilidade, serão introduzidas algumas notações:

x : variável aleatória (v.a) no espaço físico;

u : variável aleatória no espaço normal padrão não correlacionado;

$X, U$ : vetores das v.a no espaço físico e normal padrão não correlacionado;

$\mu, \sigma$ : média e desvio padrão das v.a;

$\alpha$ : cosseno diretor da v.a no espaço normal padrão não correlacionado;

$\beta \quad$ : índice de confiabilidade;

* : solução do problema;

$\mathrm{G}($.$) : função que representa a superfície limite (FS) da estrutura no$ espaço físico,

$$
\left\{\begin{array}{l}
\mathrm{G}(.)<0 \therefore \Omega_{\mathrm{f}} \\
\mathrm{G}(.)>0 \therefore \Omega_{\mathrm{s}} \\
\mathrm{G}(.)=0 \therefore \text { limite }
\end{array}\right.
$$


$\mathrm{H}($.$) : função que representa a FS da estrutura no espaço normal$ padrão;

E[.] : média.

Grande parte dos algoritmos de otimização pode se classificar em dois grupos: SQP (Sequential Quadratic Program) e SQP direct. Os algoritmos SQP trabalham com a primeira derivada da função que se deseja otimizar, $\nabla \mathrm{G}(\mathrm{X})$, enquanto os SQP direct utilizam a primeira e segunda derivada da função, $\nabla G(X)$ e $\nabla^{2} G(X)$. Os dois grupos de algoritmos têm bom desempenho, dependem da situação ou do tipo do problema. O SQP exige menor esforço matemático, pois trabalha apenas com o gradiente de $\mathrm{G}(\mathrm{X})$. Em algumas situações pode ser que o SQP direct tenha uma convergência mais rápida, uma vez que utiliza a Hessiana de $G(X)$.

Para o QRSM é melhor utilizar algoritmos de otimização SQP. No QRSM, $G(X)$ é representada por um polinômio de $2^{\circ}$ grau, cuja derivada segunda é constante, levando a uma má convergência do SQP direct. Na versão final da tese propõe-se o uso SQP + QRSM para determinar a confiabilidade de uma estrutura.

\subsection{Algoritmo de Rackwitz \& Fiessler}

Um dos algoritmos mais conhecidos no campo da confiabilidade é o algoritmo de Rackwitz \& Fiessler, que permite uma rápida determinação do ponto de projeto $\mathrm{P}^{*}$. Entretanto, existem várias situações onde esse processo contém falhas:

- $\quad$ quando houver muitos pontos de mínimo local;

- $\quad$ quando a superfície de ruína for esférica todos os pontos serão mínimos;

- $\quad$ quando a superfície de ruína apresenta ponto de sela pode haver oscilação na convergência para um mínimo global; 
- $\quad$ quando as derivadas da função de falha não forem contínuas.

Embora esse algoritmo requeira o cálculo das derivadas parciais da função de falha, função que representa a superfície de ruína da estrutura, que pode apresentar elevado custo computacional, especialmente para um número elevado de variáveis de projeto, tem bom desempenho quanto à convergência.

Levando em consideração nesse algoritmo que a reta entre a origem do sistema de coordenadas $U$ e o ponto de projeto é normal à superfície de ruína no ponto de projeto, ou seja:

$$
u_{i}^{*}=\beta \alpha_{i}^{*}
$$

O algoritmo é organizado em 5 passos:

1. Escolhe-se um ponto inicial. Geralmente:

$$
X^{0}=E[X]
$$

2. Calcula-se:

$$
\begin{aligned}
& G^{j}=G\left(x_{1}^{j}, x_{2}^{j}, \ldots, x_{n}^{j}\right) \\
& \nabla G_{i}^{j}=\left.\frac{\partial G\left(x_{1}, x_{2}, \ldots, x_{n}\right)}{\partial x_{i}}\right|^{j} \\
& \sigma_{i}^{j}=\left.\frac{\varphi\left[\Phi^{-1}\left(F_{x_{i}}\left(x_{i}\right)\right)\right]}{f_{x_{i}}\left(x_{i}\right)}\right|^{j} \\
& \mu_{i}^{j}=x_{i}-\sigma_{i} \Phi^{-1}\left[\left.F_{x_{i}}\left(x_{i}\right)\right|^{j}\right.
\end{aligned}
$$

Onde i é o índice da variável aleatória e j o índice da iteração. Ou seja:

- $\quad \mathrm{i}=1, \ldots$, no de v.a;

- $\quad \mathrm{j}=0, \ldots$, até a convergência. 
3. Calcula-se:

$$
x^{j}=\left.\sum_{i=1}^{n} \nabla G_{i} x_{i}\right|^{j} ;\left.\quad \mu_{x}\right|^{j}=\left.\sum_{i=1}^{n} \nabla G \mu_{i}\right|^{j} ;\left.\quad \sigma_{x}^{2}\right|^{j}=\left.\sum_{i=1}^{n} \nabla G_{i}^{2} \sigma_{i}^{2}\right|^{j}(6.7)
$$

4. Calcula-se:

$$
\begin{aligned}
& \alpha_{i}^{j}=\left.\frac{\nabla G_{i} \sigma_{i}}{\sigma_{x}}\right|^{j} \\
& \beta^{j}=\left.\frac{x-G-\mu_{x}}{\sigma_{x}}\right|^{j} \\
& x_{i}^{j+1}=\mu_{i}+\left.\alpha_{i} \beta \sigma_{i}\right|^{j}
\end{aligned}
$$

5. Repetem-se os passos 2, 3 e 4 até a convergência. Obtendo-se os valores finais:

$$
\beta_{\mathrm{HL}}, \alpha_{\mathrm{i}}{ }^{*}, \mathrm{u}_{\mathrm{i}}{ }^{*}, \mathrm{x}_{\mathrm{i}}{ }^{*}
$$

No caso de trabalhar diretamente no espaço normal padrão, o algoritmo resume-se à:

1. Escolha do ponto inicial. Geralmente:

$$
\mathrm{U}^{0}=\mathrm{E}[\mathrm{U}]=0
$$

2. Calcular:

$$
\begin{gathered}
H^{j}=H\left(u_{1}^{i}, u_{2}^{i}, \ldots, u_{n}^{i}\right) \\
\nabla H_{i}^{j}=\left.\frac{\partial H\left(u_{1}, u_{2}, \ldots, u_{n}\right)}{\partial u_{i}}\right|^{j}
\end{gathered}
$$


3. Calcular:

$$
\begin{aligned}
\alpha_{i}^{j} & =\frac{\nabla H_{i}^{j}}{\|\nabla\|^{j}} ; \quad \beta^{j}=\frac{H^{j}-\left\{\nabla H_{i}^{j}\right\}^{\top}\left\{u_{i}^{j}\right\}}{\| \nabla H^{j}} \\
u_{i}^{j+1} & =-\beta^{j} \alpha_{i}^{j}
\end{aligned}
$$

É necessário repetir os passos 2 e 3 até a convergência. Obtém-se os valores finais: $\beta_{\mathrm{HL},} \alpha_{i}{ }^{*}, u_{i}{ }^{*}$. Os valores de $x_{i}{ }^{*}$ são obtidos através da transformação do espaço normal padrão $u_{i}^{*}$ para espaço físico.

\subsection{Cálculo da Probabilidade de Ruína}

Os métodos mais utilizados para estimar a probabilidade de ruína de uma estrutura após a determinação do índice de confiabilidade são o FORM (First Order Reliability Method) e o SORM (Second Order Reliability Method).

\subsubsection{Método de Confiabilidade de Primeira Ordem - FORM}

O método de confiabilidade de primeira ordem fornece uma estimativa da probabilidade de ruína, linearizando a superfície de ruína (ou superfície de estado limite) no ponto de projeto $u_{i}{ }^{*}$ no espaço normal padrão, ou seja:

$$
H\left(u_{i}\right) \approx H\left(u_{i}{ }^{*}\right)+\left.\frac{\partial H\left(u_{j}\right)}{\partial u_{i}}\right|_{u_{j}=u_{j}{ }^{*}}\left(u_{i}-u_{i}{ }^{*}\right) \approx H\left(u_{i}{ }^{*}\right)+\alpha_{i}{ }^{*}\left(u_{i}-u_{i}{ }^{*}\right)
$$

Como a superfície de ruína é nula, $\mathrm{H}\left(\mathrm{u}_{\mathrm{i}}{ }^{*}\right)=0$., tem-se:

$$
\mathrm{H}\left(\mathrm{u}_{\mathrm{i}}\right) \approx \alpha_{\mathrm{i}}{ }^{*} \mathrm{u}_{\mathrm{i}}-\alpha_{\mathrm{i}}{ }^{*} \mathrm{u}_{\mathrm{i}}{ }^{*} \approx \alpha_{\mathrm{i}}{ }^{*} \mathrm{u}_{\mathrm{i}}+\beta
$$

A Equação (6.18) fornece a equação do hiperplano tangente para a superfície de ruína no ponto de projeto. 
A probabilidade de ruína é dada por:

$$
\begin{aligned}
& P_{f}=P\left[H\left(x_{i}\right) \leq 0\right]=P\left[H\left(u_{i}\right) \leq 0\right. \\
& P_{f} \cong P\left[\alpha_{i}{ }^{*} u_{i}+\beta \leq 0\right]=P\left[\alpha_{i}{ }^{*} u_{i} \leq-\beta\right]=\Phi[-\beta]
\end{aligned}
$$

onde $\Phi[$ :] é a função de distribuição acumulada padrão de apenas uma variável. Então, a aproximação FORM da probabilidade de ruína é:

$$
P_{f} \cong \Phi[-\beta]
$$

Entende-se o método em questão facilmente quando é utilizando apenas uma única variável aleatória. Portanto, nesse caso, existe o seguinte procedimento:

$$
\mathrm{i}=1 \quad \therefore \quad \alpha_{\mathrm{i}}=1 \quad \rightarrow \quad \mathrm{H}\left(\mathrm{u}_{1}\right) \approx \mathrm{u}_{1}+\beta
$$

Nessa situação, a probabilidade de ruína é dada por:

$$
\begin{aligned}
& P_{f}=P\left[H\left(x_{i}\right) \leq 0\right]=P\left[H\left(u_{1}\right) \leq 0\right. \\
& P_{f} \cong P\left[u_{1}+\beta \leq 0\right]=P\left[u_{1} \leq-\beta\right]=\Phi[-\beta]
\end{aligned}
$$

Na superfície limite. $. H\left(u_{1}\right)=0$, portanto:

$$
\begin{aligned}
& P_{f}=P\left[H\left(x_{i}\right)=0\right]=P\left[H\left(u_{i}\right)=0\right] \\
& P_{f} \cong P\left[u_{1}+\beta=0\right]=P\left[u_{1}=-\beta\right]=\Phi[-\beta]
\end{aligned}
$$

De acordo com a Equação (6.23), no caso de apenas uma variável na situação limite, o valor do índice de confiabilidade se confunde com o valor da abscissa no espaço normal padrão com o sinal trocado, conforme a Figura 6.1. 


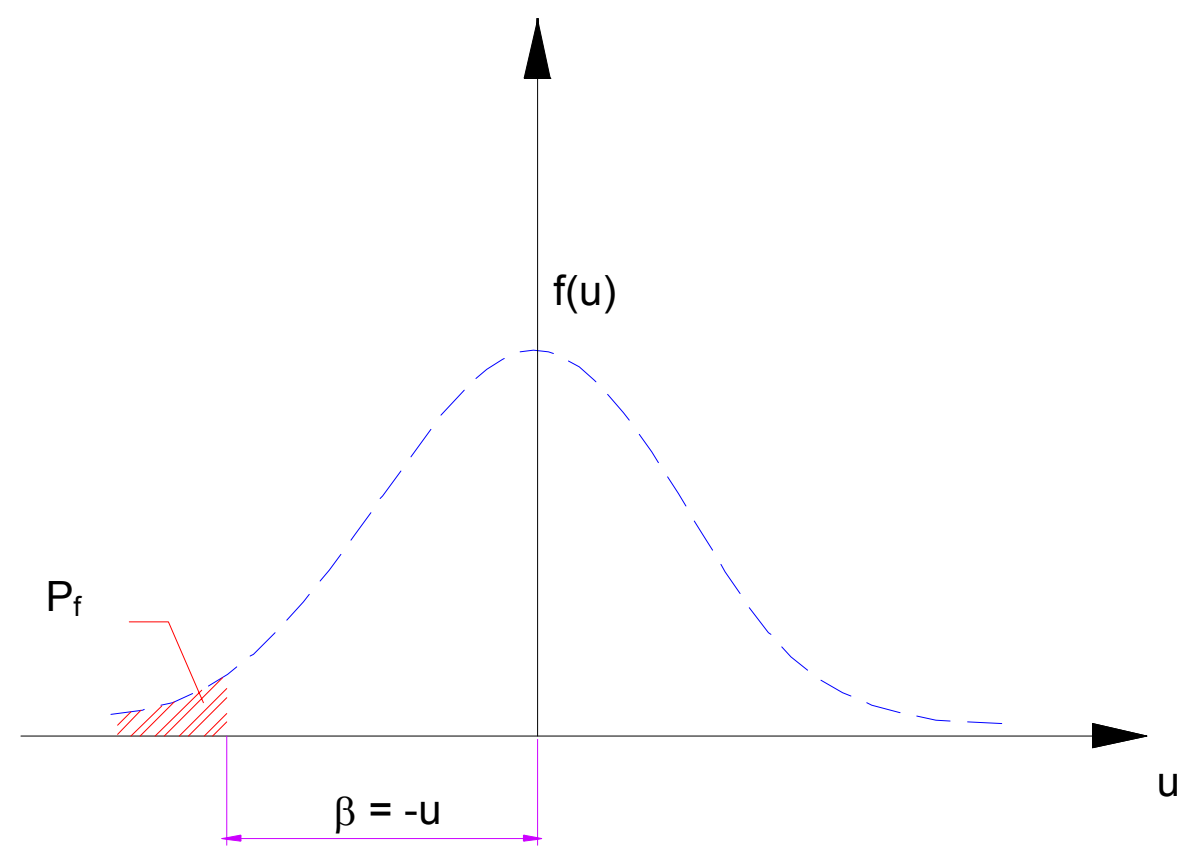

Figura 6.1 - Interpretação gráfica do FORM no caso de apenas 1 variável aleatória.

O sinal de erro na aproximação FORM depende se a superfície de ruína for côncava (a favor da segurança) ou convexa (contra a segurança), como é mostrado pela Figura 6.2. Uma aproximação FORM é suficiente se a curvatura da superfície de ruína for pequena, não há maiores problemas em linearizar a FS, e a probabilidade de ruína for um valor muito pequeno, a variação dos quantis nas extremidades da curva normal padrão não é relativamente sensível à abscissa do sistema de coordenadas. Na prática da engenharia geralmente ocorrem casos como esses. 


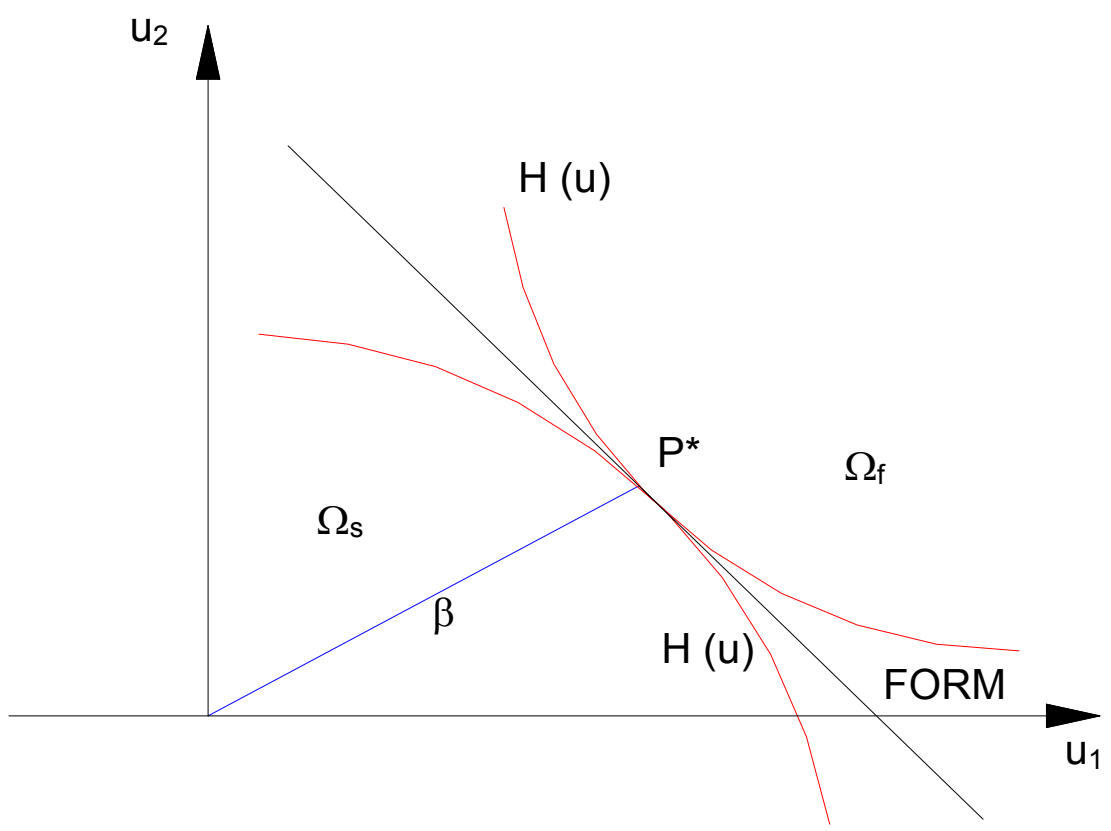

Figura 6.2 - Sinal do erro na aproximação FORM

\subsubsection{Método de Confiabilidade de Segunda Ordem - SORM}

Para melhorar a qualidade das aproximações introduz-se o SORM, baseado na idéia original do FORM. O SORM necessita de mais informações sobre a superfície de ruína em comparação com o FORM, além do índice de confiabilidade, necessário para ambos os métodos; no SORM são necessários ainda conhecimentos sobre as curvaturas principais da superfície de ruína. É utilizada uma superfície quadrática no lugar do plano tangente utilizado no FORM.

Para o ponto de projeto $\mathrm{P}^{*}$, a superfície quadrática deve ter as seguintes características:

- $\quad$ deve ser contínua e duas vezes diferenciável;

- $\quad$ deve ter o mesmo plano tangente que a superfície de ruína;

- deve ter a mesma curvatura principal que a superfície de ruína. 
São disponíveis várias aproximações para a superfície quadrática utilizada no SORM, sendo a escolha do tipo dependente do interesse da relação precisão versos custo.

\subsubsection{Aproximação por uma Hiper-esfera}

A aproximação esférica da superfície de ruína é a mais simples utilizada no SORM. Para curvatura convexa, a probabilidade de ruína sobre a fronteira é determinada por uma hiperesfera centrada na origem. Esta fronteira é dada por:

$$
\mathrm{H}\left(\mathrm{u}_{\mathrm{i}}{ }^{*}\right) \approx \sum_{\mathrm{i}=1} \mathrm{u}_{\mathrm{i}}{ }^{2}-\beta^{2}=\chi_{\mathrm{n}}^{2}-\beta^{2}
$$

A probabilidade de ruína é dada por:

$$
\begin{aligned}
& P_{f}=P\left[H\left(u_{i}\right) \leq 0\right] \\
& P_{f} \cong P\left[\chi_{n}^{2}-\beta^{2} \leq 0\right]=P\left[\chi_{n}^{2} \leq \beta^{2}\right]=1-\chi_{n}^{2}\left(\beta^{2}\right)
\end{aligned}
$$

onde. $\chi_{n}^{2}($.$) é a distribuição \chi$-quadrada com $n$ graus de liberdade.

O número de graus de liberdade é igual ao número de eixos do sistema de coordenadas da superfície de resposta da estrutura, ou seja, o número de variáveis aleatórias mais um.

\subsubsection{Hiper-esfera Excêntrica}

A melhor aproximação é obtida por hiper-esfera excêntrica com raio $R$ igual a média da curvatura principal do estado limite no ponto de projeto; o centro é então tomado pela linha reta definida pelo cosseno diretor $\alpha_{i}$. A probabilidade de ruína é aproximada por:

$$
P_{f} \cong 1-\chi_{n, \delta}^{2}\left(R^{2}\right)
$$


onde $\chi_{n, \delta}^{2}($.$) é a distribuição \chi$-quadrada com $n$ graus de liberdade e parâmetro de excentricidade $\delta=R+\beta$, sendo $\mathrm{R}$ o raio da esfera.

\subsubsection{Aproximação Assintótica}

Esses métodos são baseados em aproximações parabólicas da superfície de ruptura, geralmente apresentam boa aproximação da probabilidade de ruína. Para o seu desenvolvimento devem ser feitas duas rotações no espaço normal padrão, descritas a seguir:

- no plano de rotação, deve-se girar o eixo $\mathrm{n}$ (eixo das respostas estruturais) na direção do ponto de projeto, ou seja, na direção definida pelos cossenos diretores;

- fora do plano de rotação, deve-se girar todos os eixos, com exceção do eixo n, nas direções das curvaturas principais da FS.

Observou-se através de experiências que aproximações FORM e SORM são suficientemente precisas para problemas de engenharia.

\subsection{Formulação do Problema}

Seja $X$ o vetor de variáveis aleatórias e $G(X)$ a função de estado limite, onde:

$\mathrm{G}(\mathrm{X})>0$ é uma realização de sucesso, $x_{i} \in \Omega_{s}$, domínio de segurança;

$\ldots \mathrm{G}(\mathrm{X})<0$ é uma realização de ruína, $x_{i} \in \Omega_{f}$, domínio de ruína;

$G(X)=0$ é a função de estado limite.

Se a função aproximadora da RS é representada por $Q(X)$, a função de estado limite é determinada por: 


$$
. . \mathrm{G}(\mathrm{X})=\mathrm{Q}(\mathrm{X})-\mathrm{P}_{\mathrm{S}}
$$

onde Ps é o esforço solicitante.

O problema de confiabilidade a ser resolvido é definido por:

$$
\operatorname{Prob}\left[\left(\mathrm{Q}(\mathrm{X})-\mathrm{P}_{\mathrm{S}}\right) \leq 0\right]
$$

Para calcular o índice de confiabilidade, $\mathrm{O}$ vetor $\mathrm{X}$ das variáveis aleatórias no espaço físico passa a ser o vetor $U$ das mesmas variáveis no espaço normalizado não correlacionado através de uma transformação isoprobabilística $T$. O vetor $U$ é composto de variáveis normais padronizadas não correlacionadas, $\mathrm{N}(0,1)$. A transformação $T$ deve ser capaz de incluir todos os casos possíveis (independência, truncamento, correlação e composição), e as dimensões $\mathrm{m}$ e $\mathrm{n}$ dos espaços padronizados e físicos podem ser diferentes.

O índice de confiabilidade ( $\beta$ ) é então calculado, resolvendo-se o problema de otimização (6.29):

$$
\begin{aligned}
& . . \beta=\min \left(\sqrt{\sum_{i=1}^{m} u_{i}^{2}}\right) \\
& \text { restrição } H\left(u_{i}\right) \leq 0
\end{aligned}
$$

A solução fornece o valor do índice de confiabilidade, a coordenada $u_{i}^{*}$ do ponto de projeto $P^{*}$, e o cosseno diretor $\alpha_{i}$ da direção $P^{*}$. A interpretação gráfica do problema encontra-se na Figura 6.3. 
Espaço Físico

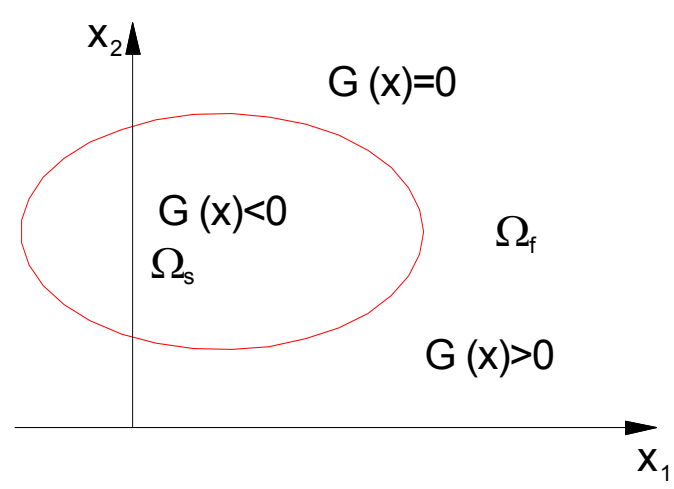

Espaço Normal Padrão

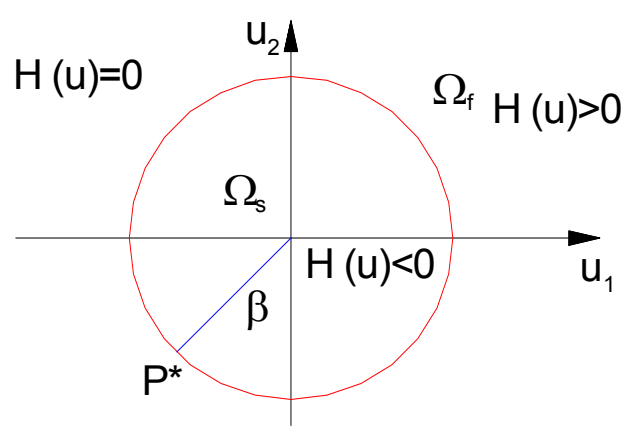

Figura 6.3 - Interpretação gráfica do problema de confiabilidade estrutural

$\beta$ é a mínima distância da origem $\mathrm{O}$ à superfície $\mathrm{H}\left(\mathrm{u}_{\mathrm{i}}\right)=0$. 


\section{OTIMIZAÇÃO DA SEÇÃO TRANSVERSAL DE UM PILAR}

\subsection{Introdução}

Atualmente, busca-se a minimização dos custos de uma estrutura satisfazendo à princípios básicos de segurança, respeitando os estados limites de ruptura e de utilização, e as imposições de normas técnicas.

Como um dos objetivos deste trabalho, tem-se o equacionamento do problema de minimização de custos da seção transversal de pilares de concreto armado em que uma função custo, sujeita à restrições de ordem prática, resulta num problema de análise não-linear, cuja solução fornece as seções transversais adequadas de pilares e as respectivas armaduras.

A solução exata do problema é alcançada através da resolução analítica do sistema de equações não-lineares gerado, a partir da aplicação do método dos multiplicadores de Lagrange e das condições de Kuhn-Tucker.

\subsection{Variáveis a serem Otimizadas}

As variáveis a serem otimizadas são as dimensões da seção transversal dos pilares e a área de armadura longitudinal. Considerando-se uma seção transversal retangular e com armadura simetricamente distribuída em duas faces da seção. Denominando-se as variáveis de $x_{i}$, para facilitar 0 procedimento matemático, têm-se as seguintes variáveis no problema, como mostrado na Figura 7.1: 
$\mathrm{x}_{1}=$ largura da seção transversal do pilar;

$\mathrm{X}_{2}=$ altura da seção transversal do pilar;

$\mathrm{x}_{3}=$ área de aço da seção transversal do pilar;

$\mathrm{X}_{4}=$ posição da linha neutra na seção transversal do pilar.

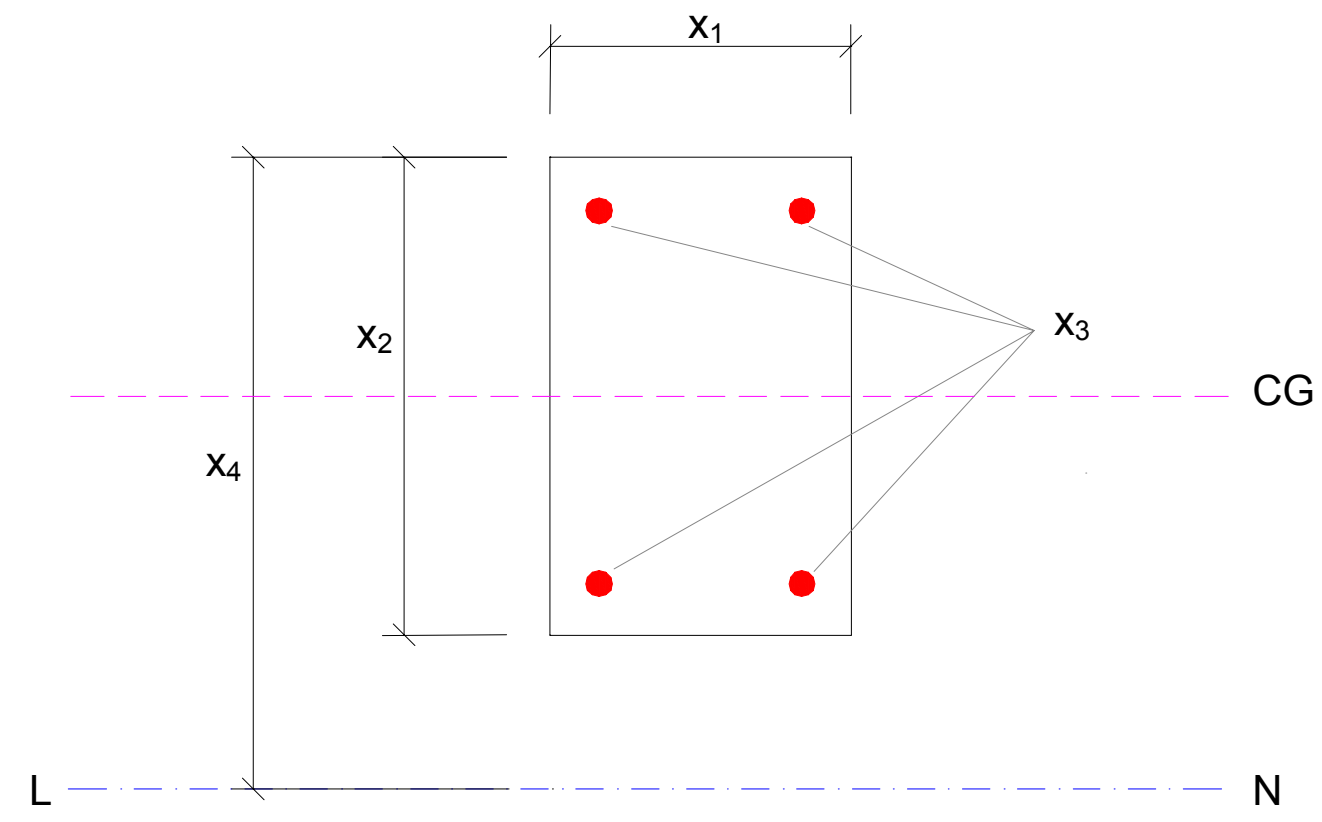

Figura 7.1 - Denominação das variáveis a serem otimizadas

Apesar da variável a ser otimizada ser apenas a área de aço longitudinal total da seção, considera-se a disposição das armaduras na seção transversal conforme ilustrado pela Figura 7.1. Esta disposição é usada para garantir o atendimento à NBR 6118, que exige um espaçamento máximo entre as barras de armadura de $40 \mathrm{~cm}$. A fim de facilitar os cálculos e por ser um recurso comumente utilizado na prática, consideram-se todas as barras da armadura com o mesmo diâmetro. Adota-se neste trabalho o aço CA-50 A por ser o mais utilizado nas estruturas.

Apesar da posição da linha neutra não estar relacionada diretamente na função objetivo como variável a ser otimizada, esta é necessária para o equilíbrio da seção, sendo por isso incluída como variável de projeto. $\mathrm{O}$ valor de $\mathrm{x}_{4}$ é definido como a distância entre a fibra mais comprimida da seção e a linha neutra da mesma. 


\subsection{Função Objetivo}

A função custo é a de menor custo por metro linear de pilar, levando-se em conta os custos com o material e mão-de-obra para a execução do pilar; incluindo nestes custos o concreto, a armadura longitudinal e a fôrma utilizada. Deste modo, tem-se a seguinte equação:

$$
F=(b \cdot h) \cdot C_{C}+A_{S} \cdot \rho_{S} \cdot C_{S}+(2 b+2 h) \cdot C_{F},
$$

onde:

F : função de custo para o pilar, por unidade de comprimento;

$\mathrm{C}_{\mathrm{C}}$ : custo do concreto, por unidade de volume;

$\mathrm{C}_{\mathrm{s}}$ : custo da armadura, por unidade de massa;

$\mathrm{C}_{\mathrm{F}}$ : custo da fôrma, por unidade de área;

b : largura da seção transversal do pilar;

h : altura da seção transversal do pilar;

$\rho_{\mathrm{S}}$ : massa específica do aço;

As : área de aço da armadura longitudinal.

Utilizam-se como unidades de medidas neste trabalho: $(\mathrm{cm})$ para unidade de comprimento e (kN) para unidade de força.

A função objetivo em função de $x$ :

$$
F(x)=\left(x_{1} \cdot x_{2}\right) \cdot C_{C}+x_{3} \cdot \rho_{S} \cdot C_{S}+\left(2 x_{1}+2 x_{2}\right) \cdot C_{F} .
$$

Para simplificar os cálculos, adota-se as seguintes constantes:

$$
\begin{aligned}
& \mathrm{C}_{1}=\mathrm{C}_{\mathrm{c}} ; \\
& \mathrm{C}_{2}=\rho_{\mathrm{S}} \cdot \mathrm{C}_{\mathrm{S}} ; \\
& \mathrm{C}_{3}=2 \mathrm{C}_{\mathrm{F}} .
\end{aligned}
$$


Assim, tem-se a função objetivo da seguinte forma:

$$
F(x)=c_{1} \cdot\left(x_{1} \cdot x_{2}\right)+c_{2} \cdot x_{3}+c_{3} \cdot\left(x_{1}+x_{2}\right)
$$

\subsection{Restrições de Equilíbrio}

Devido a incertezas na localização exata do carregamento atuante no pilar, decorrentes de falhas no projeto, imperfeições no modelo estrutural adotado, avaliações incorretas de cargas, falhas de execução e do "prumo" do pilar, a NBR-6118 não permite o dimensionamento de peças de concreto armado à compressão simples. Desta forma, consideram-se pilares sujeitos à flexão normal composta, utilizando as condições de equilíbrio em força e momento para esta situação, conforme é mostrado na Figura 7.2.
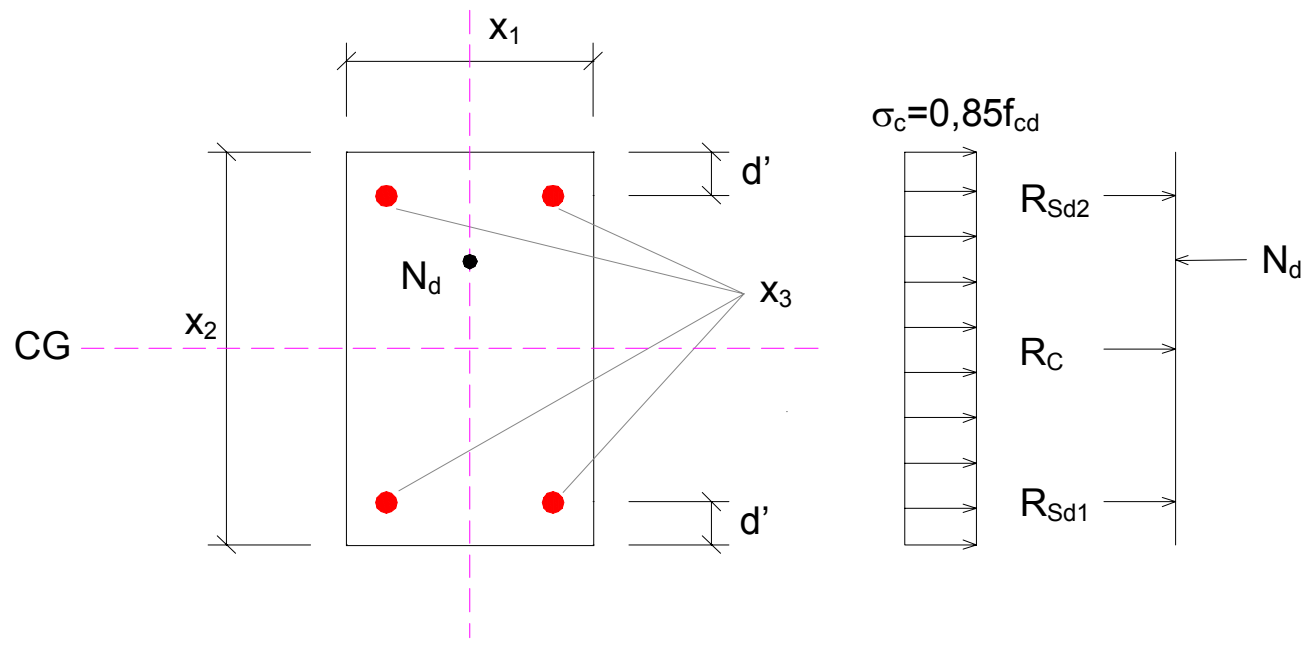
$M_{d}$

Figura 7.2 - Condições de equilíbrio

Na prática, tem-se o uso de pilares com seção constante ao longo do edifício. Desta maneira, costuma-se dimensionar a seção para resistir aos esforços na base. Como nesta situação o esforço normal é preponderante sobre o momento fletor, considera-se a seção transversal do pilar trabalhando no Domínio 5, ou seja, toda a seção transversal é comprimida. Assim, as restrições de equilíbrio ficam definidas em função de $x$, como segue. 
$\sum \mathrm{F}_{\mathrm{H}}=0$ :

$$
0,85 \cdot f_{c d} \cdot x_{1} \cdot x_{2}+\frac{x_{3}}{2}\left(\sigma_{s 1}+\sigma_{s 2}\right)-N_{d}=0
$$

$\Sigma \mathrm{M}_{\mathrm{CG}}=0$ :

$$
\frac{x_{3}}{4}\left(x_{2}-2 d^{\prime}\right) \cdot\left(\sigma_{s 1}-\sigma_{s 2}\right)-M_{d}=0
$$

onde:

d' : distância do centro da armadura até a face da seção;

$\sigma_{S i}$ : tensão atuante na armadura da camada i, sendo que as camadas são numeradas no sentido da face menos comprimida para a mais comprimida da seção.

\subsection{Restrições de Compatibilidade}

A fim de reduzir o número de restrições de igualdade na otimização, utiliza-se a restrição de compatibilidade para definir a tensão atuante nas barras de armadura, sendo este valor substituído nas restrições de equilíbrio.

Para o Domínio 5, deduz-se a equação de compatibilidade a partir da Figura 7.3, considerando-se o ponto de deformação fixa igual a $2 \%$, na faixa que dista $\frac{3}{7}$ da face mais comprimida da seção transversal. 


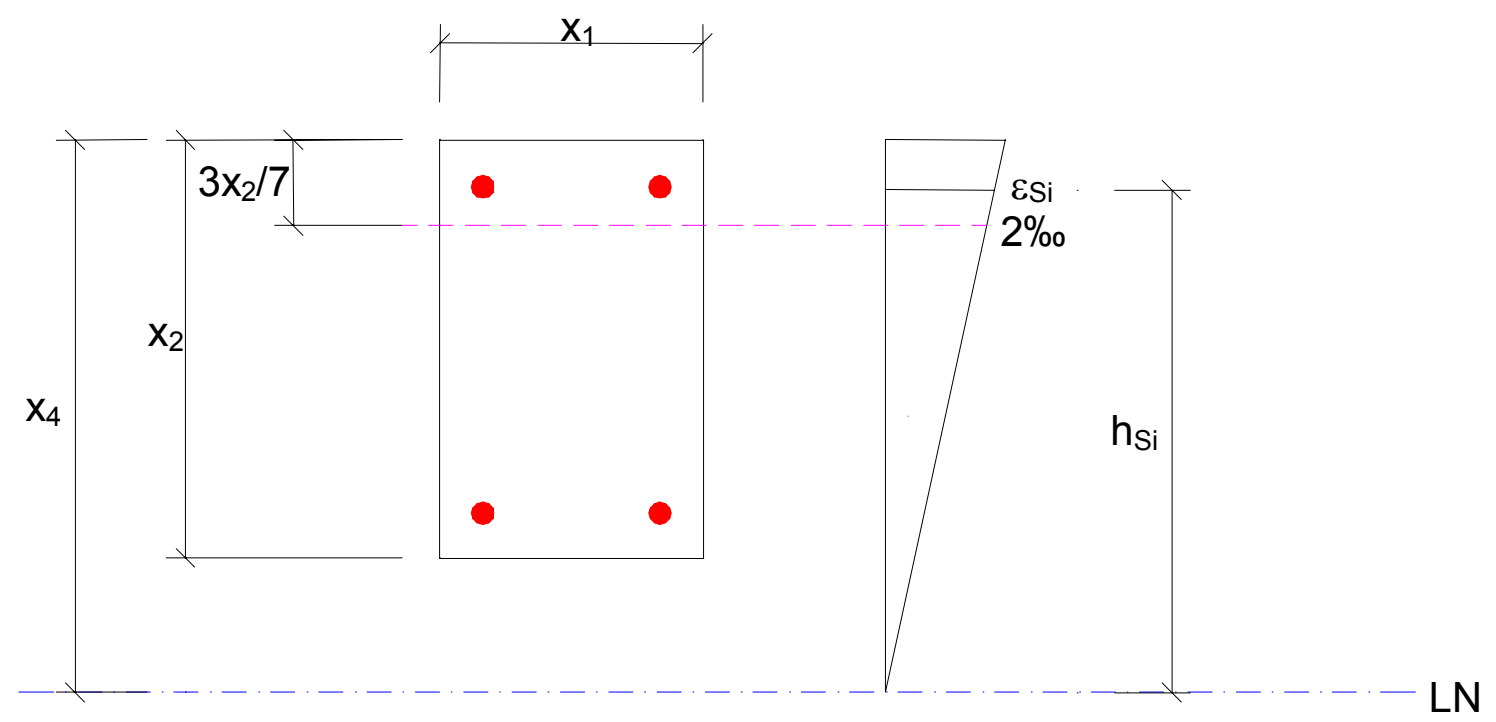

Figura 7.3 - Diagrama de deformações para o Domínio 5

Através da semelhança de triângulos, tem-se:

$$
\frac{2 \% 0}{\mathrm{x}_{4}-\frac{3}{7} \mathrm{x}_{2}}=\frac{\varepsilon_{\mathrm{Si}}}{\mathrm{h}_{\mathrm{Si}}}
$$

e após as devidas simplificações, obtém-se:

$$
\varepsilon_{\mathrm{Si}}(\% 0)=\frac{14 \cdot \mathrm{h}_{\mathrm{Si}}}{7 \mathrm{x}_{4}-3 \mathrm{x}_{2}}
$$

De acordo com a Figura 7.4, a descontinuidade do diagrama situa-se no ponto de deformação igual a 2,07\% . Como a posição da linha neutra é uma variável a ser otimizada, não se dispõe deste valor no início do processo. Desta maneira, admite-se inicialmente que as barras situadas na metade da seção transversal, entre o centro de gravidade (CG) e a face menos comprimida, terão deformação abaixo de 2,07\%o, e as barras situadas acima do CG terão deformação acima deste valor. 


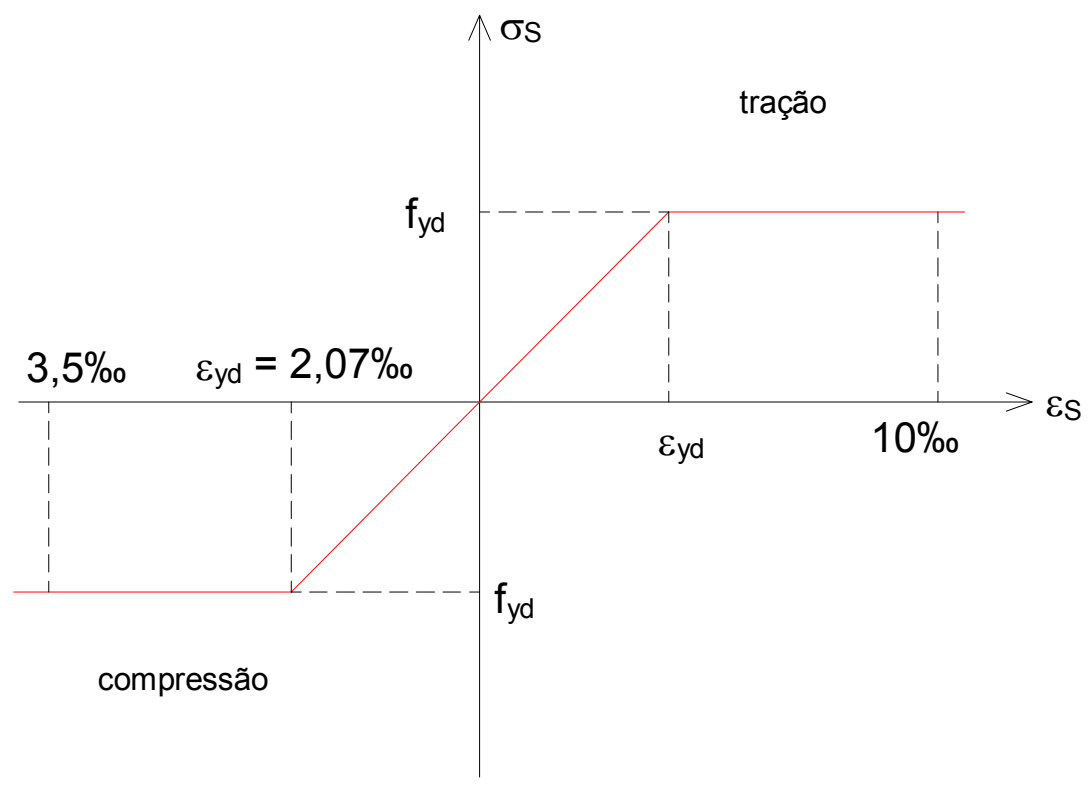

Figura 7.4 - Diagrama tensão x deformação para o aço CA-50 A

Esta hipótese é baseada no fato de que uma vez o ponto de deformação igual a $2 \%$, situa-se na faixa que dista $\frac{3}{7}$ da face mais comprimida, ou seja, pouco acima do CG. Deste modo, torna-se claro que todas as barras situadas abaixo do CG terão deformação inferior a $2 \%$, e as barras superiores só terão valor inferior a este para valores de $\mathrm{x}_{4}$ muito acima do valor de $\mathrm{x}_{2}$.

A relação tensão $x$ deformação para o aço CA-50 A, considerando-se o módulo de deformação do aço $E_{S}=21000 \mathrm{kN} / \mathrm{cm}^{2}$, pode ser dada como:

para $\varepsilon_{\mathrm{Si}}<2,07 \%$ :

$$
\begin{gathered}
\sigma_{\mathrm{Si}}=21000 \cdot \varepsilon_{\mathrm{Si}} \\
\sigma_{\mathrm{Si}}=294 \frac{\mathrm{h}_{\mathrm{Si}}}{7 \mathrm{x}_{4}-3 \mathrm{x}_{2}}
\end{gathered}
$$


para $\varepsilon_{\mathrm{Si}} \geq 2,07 \%$ :

$$
\sigma_{\mathrm{Si}}=\mathrm{f}_{\mathrm{yd}}
$$

Calculam-se as tensões $\sigma_{S i}$ nas barras, a partir da hipótese acima; substituem-se os valores nas restrições de equilíbrio; e após feitos os cálculos e simplificações possíveis, obtém-se as seguintes restrições de compatibilidade:

$$
\begin{gathered}
0,85 \cdot f_{c d} \cdot x_{1} \cdot x_{2}+\frac{x_{3}}{2}\left(294 \frac{x_{4}-x_{2}+d^{\prime}}{7 x_{4}-3 x_{2}}+f_{y d}\right)-N_{d}=0 \\
\frac{x_{3}}{4}\left(x_{2}-2 d^{\prime}\right) \cdot\left(f_{y d}-294 \frac{x_{4}-x_{2}+d^{\prime}}{7 x_{4}-3 x_{2}}+f_{y d}\right)-M_{d}=0
\end{gathered}
$$

\subsection{Restrição para garantir o Domínio 5}

Como a seção encontra-se no Domínio 5, ou seja, com toda a seção de concreto comprimida, deve-se garantir que, utilizando o diagrama de deformações simplificado retangular, a altura da seção seja menor que $80 \%$ da localização da linha neutra, ou seja:

$$
1,25 x_{2}-x_{4} \leq 0
$$

\subsection{Restrições Laterais}

Segundo a NBR 6118, a menor dimensão dos pilares não deve ser inferior a $20 \mathrm{~cm}$. A norma também solicita que a armadura longitudinal de um pilar deve ter seção transversal compreendida entre $0,8 \%$ e $3 \%$ da seção do pilar. Já para o espaçamento máximo entre as barras longitudinais, a norma indica o limite de $40 \mathrm{~cm}$. 
Portanto, de acordo com as recomendações normativas acima e a disposição da armadura, conforme a Figura 7.1, utiliza-se no problema de minimização da seção transversal de um pilar os seguintes limites mínimos e máximos para as dimensões da seção transversal e para a taxa de armadura do pilar:

$$
\begin{gathered}
20 \leq x_{1} \leq 40 \mathrm{~cm} ; \\
20 \leq x_{2} \leq 40 \mathrm{~cm} ; \\
0,8 \% \leq \rho \leq 3 \%
\end{gathered}
$$

Onde:

$\rho=$ taxa geométrica de armadura no concreto. Razão entre a área da armadura longitudinal e a área da seção transversal do pilar.

Por serem apenas limitantes de variáveis específicas, não interferindo na relação entre as variáveis, as restrições laterais não são consideradas explicitamente no processo de otimização. Estas são verificadas após cada cálculo efetuado e, no momento em que algum valor limite é atingido, a variável que atingiu este valor é retirada do processo de otimização, utilizando para a mesma o valor fixo correspondente ao limite atingido, reiniciando-se o processo de otimização.

\subsection{Resolução do Problema}

Aplicando-se à função objetivo, as restrições de equilíbrio e para garantia do Domínio 5, tem-se o seguinte problema de otimização: 
Minimizar:

$$
F(x)=c_{1} \cdot\left(x_{1} \cdot x_{2}\right)+c_{2} \cdot x_{3}+c_{3} \cdot\left(x_{1}+x_{2}\right)
$$

Sujeito à:

$$
\begin{gathered}
0,85 \cdot f_{c d} \cdot x_{1} \cdot x_{2}+\frac{x_{3}}{2}\left(294 \frac{x_{4}-x_{2}+d^{\prime}}{7 x_{4}-3 x_{2}}+f_{y d}\right)-N_{d}=0 \\
\frac{x_{3}}{4}\left(x_{2}-2 d^{\prime}\right) \cdot\left(f_{y d}-294 \frac{x_{4}-x_{2}+d^{\prime}}{7 x_{4}-3 x_{2}}\right)-M_{d}=0 \\
1,25 x_{2}-x_{4} \leq 0
\end{gathered}
$$

Aplicando-se o Lagrangeano, tem-se:

$$
\begin{aligned}
& F(x, \lambda, \mu)=c_{1} \cdot\left(x_{1} \cdot x_{2}\right)+c_{2} \cdot x_{3}+c_{3} \cdot\left(x_{1}+x_{2}\right)+ \\
& +\lambda_{1} \cdot\left[0,85 \cdot f_{c d} \cdot x_{1} \cdot x_{2}+\frac{x_{3}}{2}\left(294 \frac{x_{4}-x_{2}+d^{\prime}}{7 x_{4}-3 x_{2}}+f_{y d}\right)-N_{d}=0\right]+ \\
& +\lambda_{2} \cdot\left[\frac{x_{3}}{4}\left(x_{2}-2 d^{\prime}\right) \cdot\left(f_{y d}-294 \frac{x_{4}-x_{2}+d^{\prime}}{7 x_{4}-3 x_{2}}\right)-M_{d}=0\right]+ \\
& +\mu_{1} \cdot\left(1,25 x_{2}-x_{4}\right) .
\end{aligned}
$$

Igualando-se o gradiente do Lagrangeano a zero e aplicando-se a condição de complementaridade, encontra-se o sistema de equações apresentado a seguir que, após ser resolvido e então verificado se as soluções satisfazem as restrições inativas e as restrições laterais, fornecem os pontos de mínimo local do problema de otimização.

$$
\frac{\partial \mathrm{F}}{\partial \mathrm{x}_{1}}=\mathrm{c}_{1} \cdot \mathrm{x}_{2}+\mathrm{c}_{3}+\mathrm{c}_{4} \cdot \lambda_{1} \cdot \mathrm{x}_{2}=0
$$




$$
\begin{aligned}
& \frac{\partial F}{\partial x_{2}}=c_{1} \cdot x_{1}+c_{3}+\lambda_{1} \cdot\left[c_{4} \cdot x_{1}+0,5 \cdot x_{3} \cdot\left(-\frac{294}{7 x_{4}-3 x_{2}}+882 \frac{x_{4}-x_{2}+d^{\prime}}{\left(7 x_{4}-3 x_{2}\right)^{2}}\right)\right]+ \\
& +0,25 \cdot \lambda_{2} \cdot x_{3} \cdot\left[\left(f_{y d}-294 \frac{x_{4}-x_{2}+d^{\prime}}{7 x_{4}-3 x_{2}}\right)+\right. \\
& \left.+\left(x_{2}-2 d^{\prime}\right) \cdot\left(\frac{294}{7 x_{4}-3 x_{2}}-882 \frac{x_{4}-x_{2}+d^{\prime}}{\left(7 x_{4}-3 x_{2}\right)^{2}}\right)\right]+ \\
& +1,25 \mu_{1}=0
\end{aligned}
$$$$
\begin{aligned}
& \frac{\partial F}{\partial x_{3}}=c_{2}+0,5 \cdot \lambda_{1} \cdot\left(f_{y d}+294 \frac{x_{4}-x_{2}+d^{\prime}}{7 x_{4}-3 x_{2}}\right)+ \\
& +0,25 \cdot \lambda_{2} \cdot\left(x_{2}-2 d^{\prime}\right) \cdot\left(f_{y d}-294 \frac{x_{4}-x_{2}+d^{\prime}}{7 x_{4}-3 x_{2}}\right)=0,
\end{aligned}
$$$$
\frac{\partial \mathrm{F}}{\partial \mathrm{x}_{4}}=0,5 \cdot \lambda_{1} \cdot \mathrm{x}_{3} \cdot\left(\frac{294}{7 \mathrm{x}_{4}-3 \mathrm{x}_{2}}-2058 \frac{\mathrm{x}_{4}-\mathrm{x}_{2}+\mathrm{d}^{\prime}}{\left(7 \mathrm{x}_{4}-3 \mathrm{x}_{2}\right)^{2}}\right)+
$$$$
+0,25 \cdot \lambda_{2} \cdot\left(x_{2}-2 d^{\prime}\right) \cdot x_{3} \cdot\left(-\frac{294}{7 x_{4}-3 x_{2}}+2058 \frac{x_{4}-x_{2}+d^{\prime}}{\left(7 x_{4}-3 x_{2}\right)^{2}}\right)-\mu_{1}=0
$$$$
\frac{\partial F}{\partial \lambda_{1}}=c_{4} \cdot x_{1} \cdot x_{2}+\frac{x_{3}}{2} \cdot\left(294 \frac{x_{4}-x_{2}+d^{\prime}}{7 x_{4}-3 x_{2}}+f_{y d}\right)-N_{d}=0
$$$$
\frac{\partial F}{\partial \lambda_{2}}=\frac{x_{3}}{4} \cdot\left(x_{2}-2 d^{\prime}\right) \cdot\left(f_{y d}-294 \frac{x_{4}-x_{2}+d^{\prime}}{7 x_{4}-3 x_{2}}\right)-M_{d}=0
$$ 
$\mu_{1} \cdot\left(1,25 x_{2}-x_{4}\right)=0$

onde:

$$
\mathrm{c}_{4}=0,85 \mathrm{f}_{\mathrm{cd}}
$$

A resolução analítica do sistema de equações, como está apresentado, torna-se inexeqüível, uma vez que existem vários valores de $x_{2}$ como solução do sistema. Pelo fato de uma solução iterativa gerar um esforço computacional muito grande e $x_{2}$ variar, optou-se por trabalhar com valores fixos de $x_{2} e$ encontrar a solução analítica do sistema de equações, após a retirada do termo derivado em função de $x_{2}$.

O processo para resolução, incluindo a verificação das restrições laterais, apresenta-se da seguinte forma:

1. Faz-se $x_{2}$ variar entre os valores limites, com intervalo de variação definido pelo usuário;

2. com $x_{2}$ fixo, encontram-se as demais variáveis de projeto;

3. verifica-se se as variáveis estão dentro dos limites estabelecidos, caso os mesmos sejam ultrapassados, fixa-se o valor limite retirando a variável da otimização e retorna-se ao passo 2;

4. caso todas as variáveis estejam minimizadas e dentro dos limites, calcula-se o custo da seção minimizada e compara-se com o custo encontrado para a seção com o valor mínimo encontrado até o momento;

5. se o custo da seção for menor, esta passa a ser a seção ótima;

6. dá-se um novo incremento em $\mathrm{x}_{2}$ e reinicia-se o processo. 


\subsection{Resultados Numéricos}

Para exemplificar o processo de otimização e verificar o comportamento da função custo e das variáveis, várias séries de otimização foram executadas, considerando excentricidades atuando na direção da altura da seção de 1, 2 e $3 \mathrm{~cm}$, respectivamente.

\subsubsection{Dados}

7.9.1.1 Resistências Características dos Materiais:

$\mathrm{f}_{\mathrm{ck}}=35 \mathrm{Mpa}$

$\mathrm{f}_{\mathrm{yk}}=500 \mathrm{Mpa}$

\subsubsection{Custos dos Materiais:}

Custo do Concreto: $\mathrm{R} \$ 228,39 / \mathrm{m}^{3}$

Custo do aço: $\quad R \$ 2,73 / \mathrm{kg}$

Custo da fôrma: $\quad \mathrm{R} \$ 31,58 / \mathrm{m}^{2}$

\subsubsection{Valores Limites das Variáveis:}

$$
\begin{aligned}
& 20 \leq \mathrm{x}_{1} \leq 40 \mathrm{~cm} \\
& 20 \leq \mathrm{x}_{2} \leq 40 \mathrm{~cm} \\
& 0,008 \leq \rho \leq 0,03
\end{aligned}
$$


7.9.1.4 Resultados obtidos para excentricidade de $1 \mathrm{~cm}$ :

\begin{tabular}{|c|c|c|c|c|c|c|}
\hline $\begin{array}{c}N_{d} \\
(k N)\end{array}$ & $\begin{array}{c}M_{d} \\
(k N . c m)\end{array}$ & $\begin{array}{c}b \\
(\mathrm{~cm})\end{array}$ & $\begin{array}{c}\text { h } \\
(\mathrm{cm})\end{array}$ & $\begin{array}{c}A_{s} \\
\left(\mathrm{~cm}^{2}\right)\end{array}$ & $\begin{array}{l}\text { LN } \\
(\mathrm{cm})\end{array}$ & $\begin{array}{l}\text { Custo } \\
\text { (R\$/m) }\end{array}$ \\
\hline 250 & 250 & 20 & 20 & 3,2 & 25 & 34,46 \\
\hline 500 & 500 & 20 & 21,58 & 4,37 & 26,97 & 36,21 \\
\hline 750 & 750 & 20 & 23,95 & 5,68 & 29,93 & 38,82 \\
\hline 1000 & 1000 & 24,12 & 25,89 & 6,82 & 32,36 & 45,99 \\
\hline 1250 & 1250 & 26,50 & 27,73 & 7,79 & 34,66 & 51,21 \\
\hline 1500 & 1500 & 29,34 & 32,91 & 7,51 & 41,13 & 61,53 \\
\hline 1750 & 1750 & 31,71 & 33,04 & 8,72 & 41,3 & 65,01 \\
\hline 2000 & 2000 & 33,16 & 34,36 & 9,49 & 42,95 & 68,87 \\
\hline 2250 & 2250 & 35,74 & 37,09 & 9,72 & 46,36 & 76,49 \\
\hline 2500 & 2500 & 36,78 & 38,23 & 10,41 & 47,78 & 79,72 \\
\hline 2750 & 2750 & 40 & 40 & 10,85 & 50 & 87,30 \\
\hline 3000 & 3000 & 40 & 40 & 11,83 & 50 & 87,32 \\
\hline
\end{tabular}

Tabela 7.1 - Resultados obtidos para excentricidade de $1 \mathrm{~cm}$

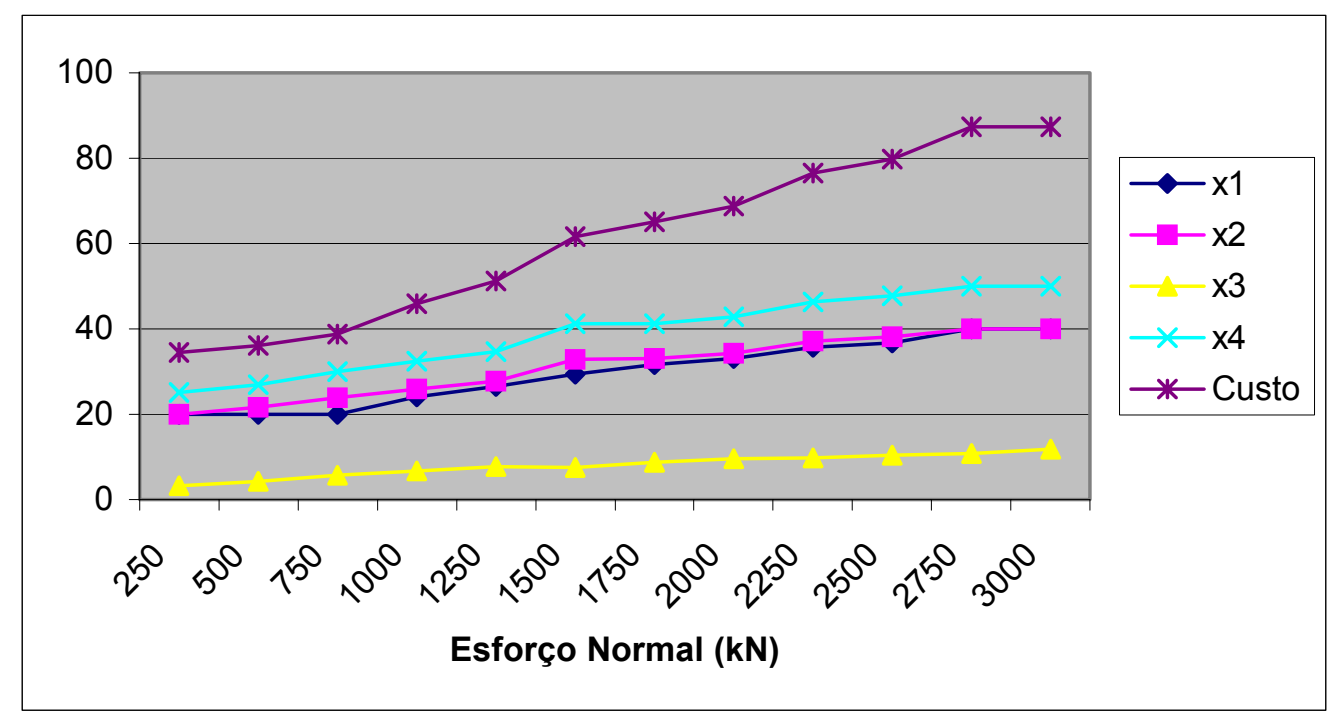

Gráfico 7.1 - Resultados obtidos para excentricidade de $1 \mathrm{~cm}$ 
7.9.1.5 Resultados obtidos para excentricidade de $2 \mathrm{~cm}$ :

\begin{tabular}{|c|c|c|c|c|c|c|}
\hline $\begin{array}{c}\mathrm{N}_{\mathrm{d}} \\
(\mathrm{kN})\end{array}$ & $\begin{array}{c}M_{d} \\
(k N . c m)\end{array}$ & $\begin{array}{c}\text { b } \\
(\mathrm{cm})\end{array}$ & $\begin{array}{c}\text { h } \\
(\mathrm{cm})\end{array}$ & $\begin{array}{c}A_{s} \\
\left(\mathrm{~cm}^{2}\right)\end{array}$ & $\begin{array}{l}\text { LN } \\
\text { (cm) }\end{array}$ & $\begin{array}{l}\text { Custo } \\
\text { (R\$/m) }\end{array}$ \\
\hline 250 & 500 & 20 & 20 & 4,88 & 25 & 34,50 \\
\hline 500 & 1000 & 20 & 22,35 & 8,33 & 27,93 & 37,13 \\
\hline 750 & 1500 & 20 & 24,76 & 10,87 & 30,95 & 39,81 \\
\hline 1000 & 2000 & 25,71 & 26,82 & 13,03 & 33,52 & 49,20 \\
\hline 1250 & 2500 & 27,82 & 28,09 & 15,33 & 35,11 & 53,48 \\
\hline 1500 & 3000 & 29,42 & 30,33 & 16,66 & 37,91 & 58,47 \\
\hline 1750 & 3500 & 31,95 & 32,89 & 17,55 & 41,11 & 65,32 \\
\hline 2000 & 4000 & 32,07 & 34,36 & 18,99 & 42,95 & 67,53 \\
\hline 2250 & 4500 & 34,47 & 36,65 & 19,74 & 45,81 & 74,19 \\
\hline 2500 & 5000 & 35,6 & 38,23 & 20,83 & 47,78 & 78,16 \\
\hline 2750 & 5500 & 40 & 40 & 21,70 & 50 & 87,53 \\
\hline 3000 & 6000 & 40 & 40 & 23,67 & 50 & 87,57 \\
\hline
\end{tabular}

Tabela 7.2 - Resultados obtidos para excentricidade de $2 \mathrm{~cm}$

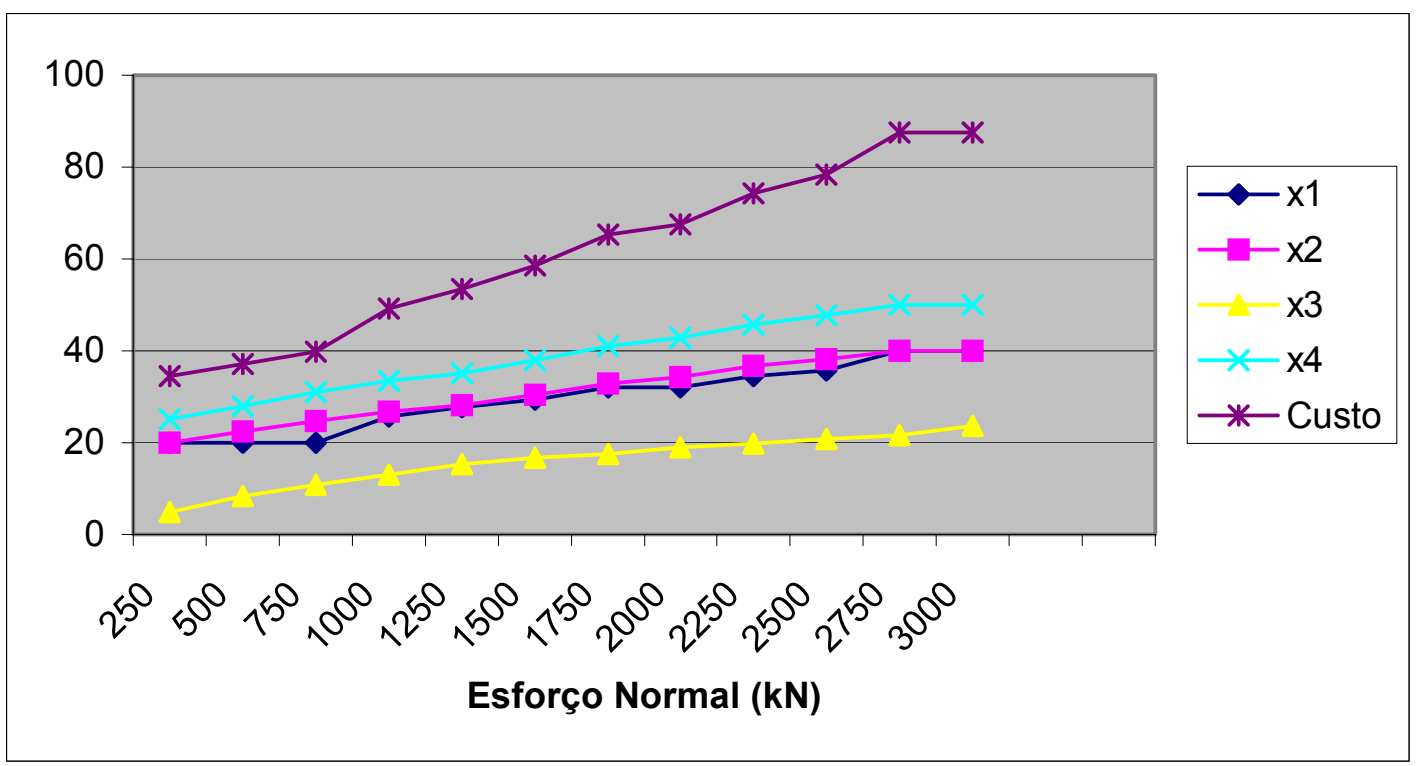

Gráfico 7.2 - Resultados obtidos para excentricidade de $2 \mathrm{~cm}$ 
7.9.1.6 Resultados obtidos para excentricidade de $3 \mathrm{~cm}$ :

\begin{tabular}{|c|c|c|c|c|c|c|}
\hline $\begin{array}{c}\mathrm{N}_{\mathrm{d}} \\
(\mathrm{kN})\end{array}$ & $\begin{array}{c}M_{d} \\
(k N . c m)\end{array}$ & $\begin{array}{c}\text { b } \\
(\mathrm{cm})\end{array}$ & $\begin{array}{c}\text { h } \\
(\mathrm{cm})\end{array}$ & $\begin{array}{c}A_{s} \\
\left(\mathrm{~cm}^{2}\right)\end{array}$ & $\begin{array}{l}\text { LN } \\
\text { (cm) }\end{array}$ & $\begin{array}{l}\text { Custo } \\
\text { (R\$/m) }\end{array}$ \\
\hline 250 & 750 & 20 & 20 & 7,32 & 25 & 34,55 \\
\hline 500 & 1500 & 20 & 23,48 & 11,68 & 29,35 & 38,43 \\
\hline 750 & 2250 & 20 & 25,01 & 16,08 & 31,26 & 40,19 \\
\hline 1000 & 3000 & 24,12 & 26,92 & 19,44 & 33,65 & 47,48 \\
\hline 1250 & 3750 & 28,50 & 29,37 & 21,70 & 36,71 & 56,14 \\
\hline 1500 & 4500 & 29,95 & 31,56 & 23,76 & 39,45 & 60,94 \\
\hline 1750 & 5250 & 31,71 & 33,86 & 25,39 & 42,32 & 66,48 \\
\hline 2000 & 6000 & 33,16 & 35,79 & 27,09 & 44,73 & 71,23 \\
\hline 2250 & 6750 & 35,74 & 37,28 & 29,00 & 46,60 & 77,18 \\
\hline 2500 & 7500 & 37,78 & 39,11 & 30,40 & 48,88 & 82,97 \\
\hline 2750 & 8250 & 40 & 40 & 32,55 & 50 & 87,76 \\
\hline 3000 & 9000 & 40 & 40 & 35,51 & 50 & 87,83 \\
\hline
\end{tabular}

Tabela 7.3 - Resultados obtidos para excentricidade de $3 \mathrm{~cm}$

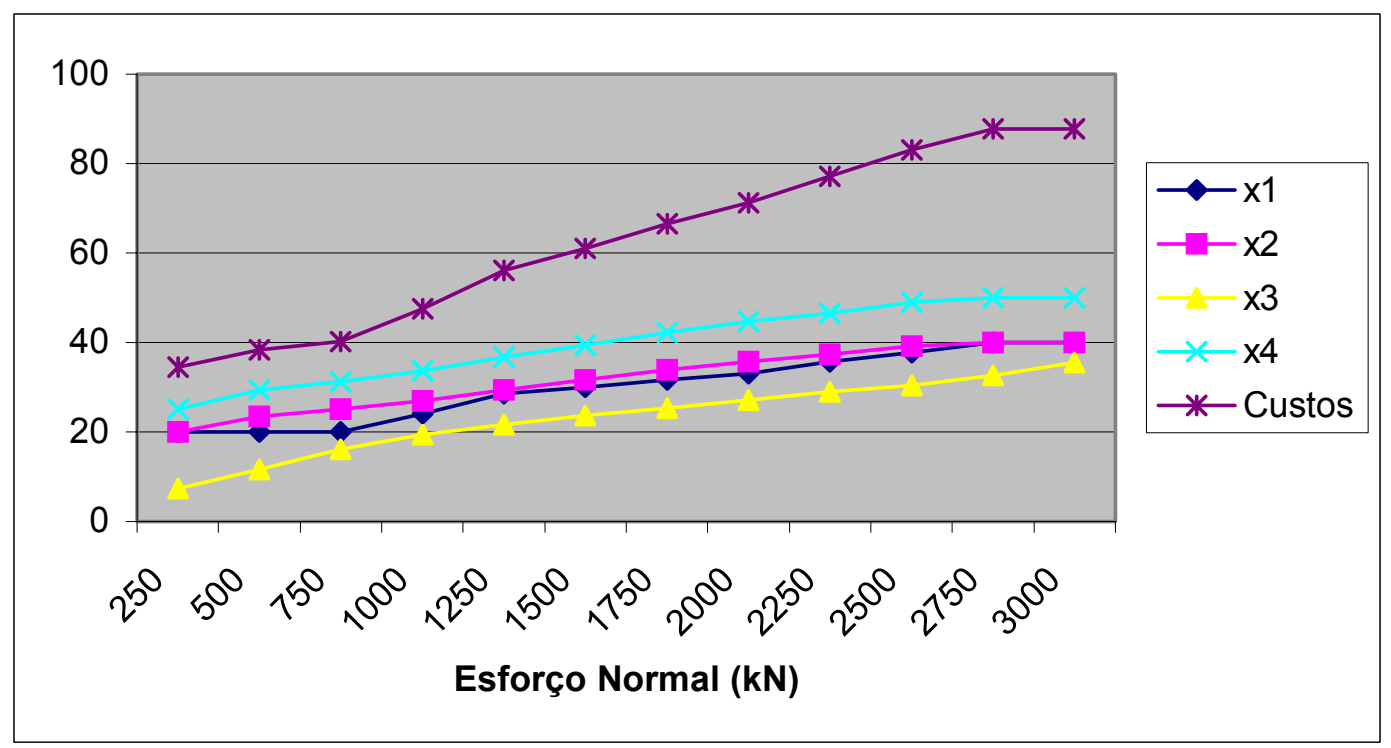

Gráfico 7.3 - Resultados obtidos para excentricidade de $3 \mathrm{~cm}$ 


\subsubsection{Análise dos Resultados}

Comparando-se os todos os resultados das três situações, nota-se primeiramente a influência da excentricidade no aumento de tais valores.

Através da observação dos resultados, pode-se notar que a restrição de desigualdade para garantir a seção totalmente comprimida está ativa para todos os valores de esforço normal. Isto comprova a hipótese que este domínio é mais indicado para valores de carregamentos mais altos.

Verifica-se nas séries de otimização o aumento na taxa de armadura no momento em que os esforços passam a ser resistidos apenas com um aumento da taxa de armadura, devido ao arranjo da armadura e as limitações das dimensões da seção de concreto.

Assim, à medida que se aumenta a área de aço utilizada, aumenta-se também a quantidade de material que não está com uma utilização otimizada, não podendo desta forma levar a uma seção econômica. Esta perda de economia pode ser bem verificada através dos Gráficos $7.1,7.2$ e 7.3, pelo sensível aumento da inclinação da curva da função custo. 


\section{CÁLCULO DO ÍNDICE DE CONFIABILIDADE}

\subsection{Introdução}

Para calcular a confiabilidade de qualquer estrutura é necessário conhecer a solicitação $S$ e a resistência $R$. No estado limite último, tanto a RS como $S$ (quando for o caso de estudo de coeficientes parciais de segurança), são definidos pela singularidade da matriz de rigidez global da estrutura, ou seja, pela instabilidade global da estrutura.

\subsection{Determinação da Solicitação (S)}

O valor atribuído a S é o coeficiente de carga (coeficiente com o qual as ações externas são multiplicadas), quando se deseja verificar a confiabilidade de uma determinada estrutura, $S$ é o coeficiente de carga equivalente ao próprio carregamento externo médio, ou seja, S é igual a unidade. Se o objetivo é calcular índices de confiabilidade para os coeficientes parciais propostos por diferentes Normas, o valor de $S$ é definido pelo coeficiente de carga que conduz a estrutura ao estado limite quando essa é calculada com resistências e solicitações alteradas por coeficientes parciais de segurança.

Para resistências de cálculo $f_{c d}$ e $f_{y d}$, calcula-se o coeficiente de carga limite global da estrutura $\left(S^{*}\right)$. Para este coeficiente obtido, a condição de projeto é dada pela inequação: $\gamma_{\mathrm{f}} \mathrm{S} \leq \mathrm{S}^{*}$. A respeito do coeficiente de carga limite, tem-se: $S=\frac{S^{*}}{\gamma_{f}^{*}}$, onde $S$ é o coeficiente de carga externa. Com esse procedimento, é possível encontrar o índice de confiabilidade $\beta$ relativo aos coeficientes parciais utilizados na prática da engenharia. 
É fácil notar que $\mathrm{S}^{*}$ é o coeficiente de carga limite determinado pelo modelo mecânico com resistência reduzida por coeficientes parciais, como $\gamma_{c} \mathrm{e}$ $\gamma_{\mathrm{s}}$, ou seja:

$$
f_{c d}, f_{y d}, V \rightarrow \text { modelo mecânico } \rightarrow S^{*}
$$

$V=$ variáveis estruturais tais como geometria, condições de contorno, etc.

Finalmente, S é determinado por:

$$
S=\frac{S^{*}}{\gamma_{f}}
$$

\subsection{Determinação da Resistência (R)}

O esforço resistente é representado pela superfície de resposta da estrutura (SR). Se a SR for elaborada com as respostas da estrutura no estado limite, pode-se dizer que a superfície de resposta se confunde com a superfície limite da estrutura.

Para encontrar a SR utiliza-se a seqüência seguinte:

1. Define-se o plano de experiência a ser utilizado.

2. Encontram-se as respostas mecânicas referentes ao plano de experiência. As respostas mecânicas são escritas em termos de coeficientes de carga que conduzem a estrutura ao estado limite. Estas respostas mecânicas são obtidas sem considerar coeficientes parciais. Os pontos, ou respostas, são definidos no espaço físico. 
3. É feita a transformação isoprobabilística passar do espaço físico para o reduzido.

4. Utiliza-se uma regressão para encontrar os coeficientes do polinômio aproximador da SR no espaço reduzido.

5. Verifica-se o erro entre a SR real e a SR aproximada.

\subsection{Cálculo do Índice de Confiabilidade $(\beta)$}

Para calcular o índice de confiabilidade utiliza-se o algoritmo de Rackwitz \& Fiessler. A superfície de ruína FS que representa o estado limite é definido pela SR subtraída da solicitação S, ou seja:

$$
\mathrm{FS}=\mathrm{SR}-\mathrm{S}
$$

\subsection{Resultados Numéricos}

Pretende-se exemplificar a determinação do índice de confiabilidade através de uma das seções otimizadas no Capítulo 7 de um pilar de concreto armado.

\subsubsection{Dados}

\subsubsection{Características Determinísticas}

Base da seção transversal, $b_{w}=25,71 \mathrm{~cm}$

Altura da seção transversal, $h=26,82 \mathrm{~cm}$

Taxa de armadura, $\rho=1,9 \%$

Excentricidade inicial, $\mathrm{e}=2 \mathrm{~cm}$

Coeficiente parcial de segurança do concreto, $\gamma_{c}=1,40$

Coeficiente parcial de segurança do aço, $\gamma_{c}=1,15$

Coeficiente parcial de segurança das ações externas, $\gamma_{c}=1,40$ 


\subsubsection{Características Estatísticas}

\begin{tabular}{|c|c|c|c|}
\hline Variável & Média & Desvio Padrão & Lei \\
\hline $\mathrm{f}_{\mathrm{c}}$ & 3,50 & 0,42 & $\mathrm{~N}$ \\
\hline $\mathrm{f}_{\mathrm{y}}$ & 50 & 3,00 & $\mathrm{~N}$ \\
\hline
\end{tabular}

\subsubsection{Determinação da Solicitação (S)}

Admite-se que a solicitação $S$ é determinada pelo coeficiente de carga que conduz a estrutura à instabilidade. Portanto, o valor de $S$ é equivalente ao coeficiente de carga que leva a estrutura à ruína quando esta é calculada considerando as resistências dos materiais reduzidas e as ações externas amplificadas por coeficientes parciais. Determina-se o valor de $S$, sendo $S=$ 1.3815. Nota-se que $S$ é um valor puramente determinístico, resultado de um simples cálculo mecânico.

\subsubsection{Determinação do Plano de Experiência (PE)}

O próximo passo é escolher um PE e desenvolvê-lo, ou seja, calcular as respostas mecânicas da estrutura para o conjunto de resistências proposto pelo PE. O plano de experiência utilizado é o plano de experiência composto.

\subsubsection{Transformação Isoprobabilística}

Para resolver o problema considera-se que a resistência do concreto é a variável aleatória número 1 e a resistência do aço a número 2. Em (8.1), $\left[X_{R}\right]$ e $\left[U_{R}\right]$ são valores atribuídos às variáveis nos espaços físico e reduzido, respectivamente. $\{R\}$ representa as respostas mecânicas no espaço físico para cada conjunto de valores físicos. 


$$
\left[X_{R}\right]=\left[\begin{array}{cc}
3,50 & 59,00 \\
2,87 & 54,50 \\
4,13 & 54,50 \\
2,24 & 50,00 \\
4,76 & 50,00 \\
2,87 & 45,50 \\
4,13 & 45,50 \\
3,50 & 41,00 \\
3,50 & 50,00
\end{array}\right] \quad\left[U_{R}\right]=\left[\begin{array}{cc}
0,00 & 3,00 \\
-1,50 & 1,50 \\
1,50 & 1,50 \\
-3,00 & 0,00 \\
3,00 & 0,00 \\
-1,50 & -1,50 \\
1,50 & -1,50 \\
0,00 & -3,00 \\
0,00 & 0,00
\end{array}\right] \quad\{R\}=\left\{\begin{array}{l}
2,850000 \\
2,676841 \\
2,853109 \\
2,365961 \\
3,081255 \\
2,432961 \\
2,872395 \\
2,617439 \\
2,679352
\end{array}\right\}
$$

\subsubsection{Determinação do Polinômio da SR}

De posse dos valores escritos em (8.1), próxima etapa é construir o polinômio que representa a SR através do método de regressão dos mínimos quadrados, o qual pode ser escrito como:

$$
\begin{aligned}
Q(U)= & 2,726+0,02763 u_{2}-0,000514 u_{2}^{2}+0,2817 u_{1}+ \\
& 0,001765 u_{1} u_{2}-0,007615 u_{1}^{2}
\end{aligned}
$$

\subsubsection{Determinação do Índice de Confiabilidade ( $\beta)$}

Como já foi determinada a SR no espaço reduzido, representada por $Q(U)$, por definição, a FS é escrita como:

$$
H(U)=Q(U)-S
$$

Próximo passo é determinar o índice de confiabilidade da iteração, que é estimado pelo algoritmo iterativo de Rackwitz \& Fiessler descrito a seguir:

1. Escolhe-se o ponto inicial: $u_{i}^{1}=0$

2. Desenvolve-se: 


$$
\begin{aligned}
& H\left(u_{i}\right)^{1}=2,726-1,3815=1,3445 \\
& \nabla H_{i}^{1}=\left.\frac{\partial H\left(u_{1}, u_{2}, \ldots, u_{n}\right)}{\partial u_{i}}\right|^{1}=\left\{\begin{array}{c}
0,2817 \\
0,02763
\end{array}\right\} \\
& \|\nabla\|^{1}=0,2831 \\
& \alpha_{i}^{1}=\frac{\nabla H_{i}^{1}}{\|\nabla\|^{1}}=\left\{\begin{array}{l}
0,9951 \\
0,0976
\end{array}\right\} \\
& \beta=\frac{H^{1}-\left\{\nabla H_{i}^{1}\right\}^{\top}\left\{u_{i}^{1}\right\}}{\|\nabla\|^{1}}=\frac{1,3445}{0,2831}=4,7492 \\
& u_{i}^{2}=-\beta^{1} \alpha_{i}^{1}=\left\{\begin{array}{l}
-4,7259 \\
-0,4635
\end{array}\right\}
\end{aligned}
$$

3. Como este foi o primeiro passo da iteração, retorna-se ao passo 2 com os novos para $\{U\}$.

4. Verifica-se o erro em $\beta$, que é determinado por:

$$
\text { erro }_{\beta}=\left|\frac{\beta^{j+1}-\beta^{j}}{\beta^{j+1}}\right|
$$

5. Caso o processo não convergiu, retornar ao passo 2. Caso contrário, fim do processo iterativo.

O processo iterativo converge quando o erro em $\beta$ atingir uma tolerância satisfatória. Aconselha-se, baseado na experiência de várias estruturas processadas, utilizar tolerância de $1 \%$ para o erro em $\beta$. 
Nesse caso particular, a convergência ocorre no terceiro passo do processo iterativo. Os valores de $\beta$ e U são:

$$
\beta=4,6947 \quad U=\left\{\begin{array}{c}
-4,6731 \\
-0,4358
\end{array}\right\}
$$

Percebe-se que até o instante, foi estimado o índice de confiabilidade para uma RS arbitrária e o procedimento é válido apenas para RS construídas em torno do ponto de projeto. Portanto, necessita-se construir outras RS até que em duas RS consecutivas o índice de confiabilidade satisfaça uma determinada tolerância. O procedimento segue, deve ser construída outra RS trocando o par de valores de resistência mais distante da solução pelo equivalente aos valores de $\{U\}$ encontrados em (8.2). Portanto, os novos valores para $\left[X_{R}\right]$ e $\left[U_{R}\right]$ são:

$$
\left[X_{R}\right]=\left[\begin{array}{cc}
3,50 & 59,00 \\
2,87 & 54,50 \\
4,13 & 54,50 \\
2,24 & 50,00 \\
1,53 & 48,7226 \\
2,87 & 45,50 \\
4,13 & 45,50 \\
3,50 & 41,00 \\
3,50 & 50,00
\end{array}\right] \quad\left[U_{R}\right]=\left[\begin{array}{cc}
0,00 & 3,00 \\
-1,50 & 1,50 \\
1,50 & 1,50 \\
-3,00 & 0,00 \\
-4,6731 & -0,4258 \\
-1,50 & -1,50 \\
1,50 & -1,50 \\
0,00 & -3,00 \\
0,00 & 0,00
\end{array}\right] \quad\{R\}=\left\{\begin{array}{l}
2,850000 \\
2,676841 \\
2,853109 \\
2,365961 \\
1,342085 \\
2,432961 \\
2,872395 \\
2,617439 \\
2,679352
\end{array}\right\}
$$

Reinicia todo processo de cálculo até que os valores dos índices de confiabilidade para duas FS consecutivas sejam iguais a menos de uma tolerância preestabelecida. Conhecido o valor de $\beta$ facilmente obtém-se a probabilidade de ruína da estrutura utilizando métodos como o FORM ou o SORM. 


\subsubsection{Análise dos Resultados}

Através dos resultados obtidos no Capítulo 7 para as seções transversais ótimas, calculou-se o índice de confiabilidade para diferentes planos de experiência, conforme mostra a Tabela 8.1 e Tabela 8.2.

\begin{tabular}{|c|c|c|c|c|c|}
\hline $\begin{array}{l}\text { Excentricidade } \\
\text { (cm) }\end{array}$ & Solicitações & $\begin{array}{c}\text { Seção Ótima } \\
(\mathrm{cm})\end{array}$ & $\begin{array}{c}A_{s} \\
\left(\mathrm{~cm}^{2}\right)\end{array}$ & $\begin{array}{c}\text { Plano de } \\
\text { Experiência }\end{array}$ & $\beta$ \\
\hline \multirow{5}{*}{1} & \multirow{5}{*}{$\begin{array}{c}N_{d}=750 \mathrm{kN} \\
M_{d}=750 \mathrm{Kn} \cdot \mathrm{cm}\end{array}$} & \multirow{5}{*}{$20 \times 23,98$} & \multirow{5}{*}{10,45} & Estrela & 4,2025 \\
\hline & & & & Estrela $45^{\circ}$ & 4,2018 \\
\hline & & & & Fatorial Completo & 4,2011 \\
\hline & & & & Mínimo & 4,2017 \\
\hline & & & & Composto & 4,2042 \\
\hline \multirow{5}{*}{2} & \multirow{5}{*}{$\begin{array}{c}N_{d}=1500 \mathrm{kN} \\
M_{d}=2500 \mathrm{kN} \cdot \mathrm{cm}\end{array}$} & \multirow{5}{*}{$29,34 \times 32,91$} & \multirow{5}{*}{17,55} & Estrela & 4,6033 \\
\hline & & & & Estrela $45^{\circ}$ & 4,6048 \\
\hline & & & & Fatorial Completo & 4,6053 \\
\hline & & & & Mínimo & 4,6723 \\
\hline & & & & Composto & 4,6947 \\
\hline \multirow{5}{*}{3} & \multirow{5}{*}{$\begin{array}{c}\mathrm{N}_{\mathrm{d}}=2500 \mathrm{kN} \\
\mathrm{M}_{\mathrm{d}}=4000 \mathrm{kN} \cdot \mathrm{cm}\end{array}$} & \multirow{5}{*}{$35,6 \times 38,23$} & \multirow{5}{*}{25,39} & Estrela & 4,7315 \\
\hline & & & & Estrela $45^{\circ}$ & 4,7497 \\
\hline & & & & Fatorial Completo & 4,7271 \\
\hline & & & & Mínimo & 4,8126 \\
\hline & & & & Composto & 4,6392 \\
\hline
\end{tabular}

Tabela 8.1 - Índices de Confiabilidade para Seções Ótimas

Pode-se observar através dos resultados acima que o PE Fatorial Completo e o PE Composto conduziram o procedimento a uma rápida convergência, demonstrando ótimo desempenho e resultados satisfatórios. 


\begin{tabular}{|c|c|c|c|c|c|c|c|c|c|c|}
\hline \multirow{2}{*}{$\begin{array}{c}\text { Plano de } \\
\text { Experiência }\end{array}$} & \multicolumn{2}{|c|}{ Solictação } & \multicolumn{3}{|c|}{ Valores Ótimos } & \multicolumn{5}{|c|}{ Valores de Projeto } \\
\hline & $\begin{array}{l}\mathrm{Nd} \\
(\mathrm{kN})\end{array}$ & $\begin{array}{c}\text { Md } \\
(\mathrm{kN} . \mathrm{cm})\end{array}$ & $\begin{array}{c}\text { Seção } \\
\text { (cm) }\end{array}$ & $\begin{array}{c}A_{S} \\
\left(\mathrm{~cm}^{2}\right)\end{array}$ & $\beta$ & $\begin{array}{c}\text { Seção } \\
(\mathrm{cm})\end{array}$ & $\begin{array}{c}A_{s} \\
\left(\mathrm{~cm}^{2}\right)\end{array}$ & $\beta$ & $\begin{array}{c}A_{S} \\
\left(\mathrm{~cm}^{2}\right)\end{array}$ & $\beta$ \\
\hline Estrela & \multirow{15}{*}{750} & \multirow{5}{*}{750} & \multirow{5}{*}{$20 \times 23,95$} & \multirow{5}{*}{5,68} & 4,2025 & \multirow{15}{*}{$20 \times 25$} & \multirow{5}{*}{5,36} & 4,2025 & \multirow{5}{*}{$\begin{array}{c}4 \varnothing 16 \\
8,04\end{array}$} & 4,4143 \\
\hline Estrela $45^{\circ}$ & & & & & 4,2018 & & & 4,2018 & & 4,4129 \\
\hline Fatorial Completo & & & & & 4,2011 & & & 4,2011 & & 4,4118 \\
\hline Mínimo & & & & & 4,2017 & & & 4,2017 & & 4,4123 \\
\hline Composto & & & & & 4,2042 & & & 4,2042 & & 4,4156 \\
\hline Estrela & & \multirow{5}{*}{1500} & \multirow{5}{*}{$20 \times 24,76$} & \multirow{5}{*}{10,87} & 4,6033 & & \multirow{5}{*}{10,73} & 4,6033 & \multirow{5}{*}{$\begin{array}{c}4 \varnothing 20 \\
12,57\end{array}$} & 4,6983 \\
\hline Estrela $45^{\circ}$ & & & & & 4,6048 & & & 4,6048 & & 4,6996 \\
\hline Fatorial Completo & & & & & 4,6053 & & & 4,6053 & & 4,6999 \\
\hline Mínimo & & & & & 4,6723 & & & 4,6723 & & 4,7154 \\
\hline Composto & & & & & 4,6947 & & & 4,6947 & & 4,7231 \\
\hline Estrela & & \multirow{5}{*}{2250} & \multirow{5}{*}{$20 \times 25,01$} & \multirow{5}{*}{16,08} & 4,7315 & & \multirow{5}{*}{16,09} & 4,7315 & \multirow{5}{*}{$\begin{array}{c}4 \varnothing 32 \\
32,16\end{array}$} & 4,9517 \\
\hline Estrela $45^{\circ}$ & & & & & 4,7497 & & & 4,7497 & & 4,9607 \\
\hline Fatorial Completo & & & & & 4,7271 & & & 4,7271 & & 4,9482 \\
\hline Mínimo & & & & & 4,8126 & & & 4,8126 & & 4,9967 \\
\hline Composto & & & & & 4,6392 & & & 4,6392 & & 4,7291 \\
\hline
\end{tabular}

Tabela 8.2 - Índices de Confiabilidade para Seções Ótimas

Comparando-se agora os valores dos índices de confiabilidade para novas dimensões ótimas, mostrados na Tabela 8.2 , com os resultados do índice de confiabilidade para dimensões usualmente utilizadas em projetos, pode-se verificar que estes são muito próximos. Observa-se também que o PE Fatorial Completo e o PE Composto conduziram o procedimento a uma rápida convergência, demonstrando ótimo desempenho e resultados satisfatórios.

Conclui-se que o Método de Superfície de Resposta, utilizando polinômios de ordem baixa como função aproximadora representativa da superfície de ruína, é recomendado para calcular o índice de confiabilidade $\beta$ em estruturas de concreto armado. Entretanto, esse método apresenta uma forte dependência do plano de experiência adotado. O custo computacional apresentado por esse método é bastante reduzido, apresentando as respostas com precisão satisfatória.

O ponto crítico dos métodos baseados em superfícies de resposta é o custo computacional do modelo mecânico. Qualquer melhoria em diminuir o 
número de pontos dos planos de experiência, como conseqüência diminuir o número de respostas mecânicas necessárias para verificar-se a convergência do método é de grande aceitação no meio técnico. Portanto, caso deseje-se melhorar um MSR devesse tentar diminuir o número de respostas mecânicas para construção da SR. Isso pode ser feito otimizando a distribuição dos pontos dos PE.

Com relação à obtenção do polinômio aproximador das respostas mecânicas, acredita-se que o método dos mínimos quadrados é o mais adequado. Finalmente, acredita-se que os MSR são adequados para estimar índices de confiabilidade em qualquer tipo de estrutura, composta por qualquer material ou conjunto de materiais de comportamento linear ou não-linear. 


\section{CONCLUSÕES}

Neste trabalho foi desenvolvido um programa para a otimização do dimensionamento de pilares de concreto armado e cálculo do índice de confiabilidade. É possível que a otimização global da estrutura traga resultados mais favoráveis, mas a dificuldade de implementação e o custo computacional para se definir uma estrutura ótima, utilizando um processo global e trabalhando com elementos de comportamento como o concreto, faz com que este tipo de otimização, por aproximação combinada, se torne de mais atraente implementação. A função objetivo foi uma função de menor custo por unidade de comprimento do elemento. Foram consideradas restrições de forma a garantir o equilíbrio e a compatibilidade das seções, além de restrições laterais que trazem limitações de ordem prática ou normativa para as estruturas.

Para os pilares, as restrições laterais adotadas foram a de garantir a seção totalmente comprimida, pois foi prevista a otimização da seção mais solicitada da edificação que, geralmente, se encontra nesta situação, e as limitações de ordem prática das dimensões da seção transversal e da taxa de armadura. Estas limitações de ordem práticas também foram feitas em separado do processo de otimização em si, pois se tratavam apenas de limitações em variáveis isoladas, não afetando a relação entre as variáveis.

No estudo das seções dos pilares, o fato de se trabalhar com a seção de concreto totalmente comprimida, onde este material é mais eficiente e com a disposição simétrica da armadura, faz com que os esforços sejam melhor combatidos com a utilização de maior área de concreto por ser o material melhor empregado nesta situação. 
Os valores das variáveis adotados como dados de entrada da estrutura tem pouco significado na seção ótima dos elementos estruturais. O fato de se aplicar um valor inicial muito diferente da realidade pode ser prejudicial apenas na velocidade de convergência do programa, pois irão ocorrer grandes variações ao longo das primeiras iterações, de forma a provocar uma grande redistribuição de esforços, fazendo com que sejam necessárias mais iterações a fim de se chegar à seção otimizada.

Uma vez que as perturbações provocadas pelas grandes variações das dimensões se dissipem, a estrutura tende a convergir rapidamente para os valores ótimos. Devido a estas variações, é recomendável que se defina um número máximo de iterações compatível com as incertezas quanto à proximidade dos dados de entrada em relação aos valores ótimos da estrutura. Quanto mais incerto se estiver da distância entre os valores adotados e os valores ótimos, maior deve ser o número máximo de iterações, não sendo recomendado um número de iterações máximo menor que vinte.

Há dois aspectos principais a se considerar em um modelo de projeto adequado para ser aplicado às estruturas. O primeiro aspecto importante é a possibilidade do modelo de poder quantificar a confiabilidade das estruturas no estado limite de utilização e no estado limite último, garantindo assim uma probabilidade de falha pré-definida.

O segundo aspecto importante é com relação ao modelo mecânico, onde as condições de equilíbrio e compatibilidade geométrica devem ser satisfeitas junto com os modelos adotados para descrever o comportamento dos materiais. O modelo mecânico deve ser escolhido de tal forma que represente o mais fielmente possível o comportamento atual da estrutura.

Por outro lado, o modelo de confiabilidade deve ser escolhido para estimar precisamente probabilidades de falha, levando-se em consideração o aspecto computacional que pode restringir sua aplicabilidade. Além de estimar a confiabilidade das estruturas, verificou-se que os métodos adotados também 
podem ser utilizados para otimizar a estrutura garantindo um mínimo de segurança.

O Método de Superfícies de Respostas conduz a excelentes resultados, com rápida convergência e consistência. Esse método tem suas desvantagens, como qualquer outro, a principal delas é a extrema dependência do plano de experiência adotado para o desenvolvimento. Uma outra desvantagem para o engenheiro é que exige o emprego de conhecimentos matemáticos mais complexos.

Problemas com elevado número de variáveis aleatórias conduzem a trabalhar no espaço com um número elevado de dimensões. Isso pode trazer problemas para definição do PE escolhido para governar o algoritmo.

O MSR associado a um algoritmo de otimização pode ser utilizado para estimar o índice de confiabilidade da estrutura. Métodos de Confiabilidade de Primeira e Segunda Ordem, FORM e SORM, respectivamente, são algoritmos que podem estimar a probabilidade de falha da estrutura quando já se conhece seu índice de confiabilidade.

\section{Propostas para Pesquisas Futuras}

Algumas sugestões para a continuidade da pesquisa na área da otimização estrutural podem ser feitas em função das observações dos resultados da análise numérica efetuada: adotar diferentes distribuições de armaduras; adotar as seções trabalhando em todos os domínios de cálculo de forma a deixar livre a variação de seções dos pilares, fazendo com que possam ser aplicados em diferentes tipos de construção, e submetidas a variados casos de carga; otimizar seções de pilares trabalhando a flexão oblíqua, deixando sua variação mais ampla; utilizar o pilar trabalhando a flexão oblíqua em um programa para otimização de pórticos planos e espaciais, realizando desta forma a otimização da estrutura de um edifício como um todo. 
Com relação a trabalhos futuros na área da confiabilidade estrutural, o MSR pode ser melhorado no que diz respeito à definição de Planos de Experiência. Sabe-se que qualquer ganho no custo computacional é de grande importância, e a maior contribuição do MSR para o custo computacional vem do tempo de processamento da resposta mecânica. Portanto, quanto menor o número de respostas mecânicas necessárias para desenvolver o MSR, melhor será. Uma possibilidade é utilizar processos de otimização para definir os pontos dos PE.

Nota-se que o uso de coeficientes parciais constantes como indicam as normas atuais não é o mais indicado para uma probabilidade de falha uniforme. Um procedimento possível para trabalhos futuros é a análise da confiabilidade e conseqüentemente nível de segurança que eles oferecem. É necessário também se definir os parâmetros estatísticos (desvio padrão e distribuições) para os modelos que garantam uma confiabilidade mais uniforme e aceita pela sociedade. Esse estudo deve ser feito separadamente para os Estados limites Último e de Serviço. 


\section{REFERÊNCIAS}

ADAMU, A.; KARIHALOO, B.L. (1994a). Minimum cost design of RC beams using DCOC, Part I: Beams with freely-varying cross section. Structural Optimization, v.7, n.4, p.237-251, jun.

ADAMU, A.; KARIHALOO, B.L. (1994b). Minimum cost design of RC beams using DCOC, Part II: Beams with uniform cross section. Structural Optimization, v.7, n.4, p.252-259, jun.

ADAMU, A.; KARIHALOO, B.L. (1995b). Minimum cost design of RC frames using DCOC method, Part I: Columns under uniaxial bending actions. Structural Optimization, v.10, n.1, p.16-32, ago.

ADAMU, A.; KARIHALOO, B.L. (1995a). Minimum cost design of RC frames using DCOC method, Part II: Columns under uniaxial bending actions. Structural Optimization, v.10, n.1, p.33-39, ago.

AKTAS, E.; MOSES, F.; GHOSN, M. (2001). Cost and safety optimization of structural design specifications. Reliability Engineering and System Safety, v.73, p.205-212.

AL-SALLOUM, Y.A.; SIDDIQI, G.H. (1994). Cost-optimum design of reinforced concrete beams. ACl Structural Journal, v.91, n.6, p.647-655, nov/dez.

AMERICAN SOCIETY OF CIVIL ENGINEERS (1997). Guide to structural optimization. New York: ASCE.

ANG, A.H.S.; TANG, W.H. (1974). Probability Concepts in Engineering Planning and Design. New York: John Wiley.v.1. 
ANG, A.H.S.; TANG, W.H. (1985). Probability Concepts in Engineering Planning and Design. New York: John Wiley.v.2.

ANTÓNIO, C.A.C. (2001). A hierarchical genetic algorithm for reliability based design of geometrically non-linear composite structures. Composite Structures, v.54, p.37-47.

ARORA, J.S. (1980). Analysis of optimality criteria and gradient projection methods for optimal structural design. Computer Methods in Applied Mechanics and Engineering, v.23, p.185-213.

ASSOCIAÇÃO BRASILEIRA DE NORMAS TÉCNICAS (1978). NBR 6118: Projeto de estruturas de concreto armado. Rio de Janeiro.

BALLING, R.J.; YAO, X. (1997). Optimization of reinforced concrete frames. Journal of Structural Engineering, ASCE, v.123, n.2, p.193-202, fev.

BARNETT, R.L. (1961). Minimum weight design of beams for deflection. Journal of Engineering Mechanics Division, ASCE, v.87, n.1, p.75-109, jan.

BAZANT, Z.P.; CEDOLIN, L.; TAGGARA, M.R. (1991). New method of analysis of slender columns. ACI Structural Journal, v.88, p.391-401.

BAZARAA, M.; SHERALI, H.D.; SHETTY, C.M. (1993). Nonlinear programming: theory and applications. 2.ed. New York: John Wiley.

BERNARDO, T.R.R. (1999). Contribuição à análise de confiabilidade em pavimentos de edifícios de concreto armado. Dissertação (Mestrado) - Escola de Engenharia de São Carlos, Universidade de São Paulo, São Carlos. 1999.

BICKEL, P.J.; DOKSUM, K.A. (1977). Mathematical Statistics, Basic Ideas and Selected Topics. Holden Day Inc. 
BIONDINI, F.; BONTEMPI, F.; MALERBA, P.G. (1998). Optimization of strutand-tie models in reinforced concrete structures. ASCO, p.115-122.

BLJUGER, D.V.; YANKELEVSKY, D. (1996). Optimization problem solution in reliability analysis of reinforced concrete structures. Computers e Structures, v.60, n.3, p. 351-355.

BOLDRINI, J.L. et al. (1980). Álgebra Linear. 3.ed. São Paulo: Harbra.

CAMP, C.; PEZESHK, S.; CAO, G. (1998). Optimized design of twodimensional structures using a genetic algorithm. Journal of Structural Engineering, ASCE, v.124, n.5, p.551-559, mai.

CEREDA, R.L.D.; MALDONADO, J.C. (1987). Introdução ao Fortran 77 para microcomputadores. São Paulo: McGraw-Hill.

CHAKRABARTY, B.K. (1992). A model for optimal design of reinforced concrete beam. Journal of Structural Engineering, ASCE, v.118, n.11, p.32383242, nov.

CHAVES, E.W.W. (1997). Fortran powerstation 4.0. 1.ed. São Carlos.

COELLO, C.C.; HERNÁNDEZ, F.S.; FARRERA, F.A. (1997). Optimal design of reinforced concrete beams using genetic algorithms. Expert systems with applications, v.12, n.1, p.101-108.

DEGROOT, M.H. (1975). Probability and Statistics. Addison-Wesley.

DITLEVSEN, O.; MADSEN, H.O. (1996). Structural Reliability Methods. New York: John Wiley.

ELLINGWOOD, B.R. (1996). Reliability based condition assessment and LRFD for existing structures. Structural Safety, v.18, n.2, p.67-80. 
ESTES, A.C.; FRANGOPOL, D.M. (2001). Minimum expected cost oriented optimal maintenance planning for deteriorating structures: application to concrete bridge decks. Reliability Engineering and System Safety, v.73, p.281291.

FADDAE, M.J.; GRIERSON, D.E. (1996). Design optimization of 3D reinforced concrete structures. Structural Optimization, v.12, n.2/3, p.127-134, out.

FOX, R. (1973). Optimization methods for engineering design. Reading: Addison-Wesley.

FRANCHIN, P.; DITLEVSEN, O.; KIUREGHIAN, A.D. (2002). Model correction factor method for reliability problems involving integrals of non-Gaussian random fields. Probabilistic Engineering Mechanics, v.17, p.109-122.

FRANGOPOL, D.M.; IDE, Y.; SPACONE, E.; IWAKI, I. (1996). A new look at reliability of reinforced concrete columns. Structural Safety, v.18, n.2, p.123150.

FREUDHENTAL, A.M. (1947). The Safety of Structures. ASCE.

FRIEL, L.L. (1974). Optimum singly reinforced concrete sections. ACI Journal, v.71, n.11, p.556-558, nov.

FRTZCHE, H. (1978). Programação não-linear: análise e métodos. São Paulo: Edgard Blücher.

GARBATOV, Y.; SOARES, C.G. (2001). Cost and reliability based strategies for fatigue maintenance planning of floating structures. Reliability Engineering and System Safety, v.73, p.293-301.

GILL, P.; MURRAY, W.; WRIGHT, M.H. (1981). Practical optimization. London: Academic Press. 
GOLDBERG, D.E.; SAMTAMI, M.P. (1986). Engineering optimization via genetic algorithm. Proceedings of $9^{\text {th }}$ conf. Eletronic Computation, ASCE, p.471-482.

GUAN, X.L.; MELCHERS, R.E. (2002). Effect of response surface parameter variation on structural reliability estimates. Structural Safety.

HAFTKA, R.T.; GÜRDAL, Z. (1992). Elements of structural optimization. Dordrecht: Kluwer Academic Publishers.

HASSANAIN, M.A.; LOOV, R.E. (1999). Design of prestressed girder bridges using high performance concrete - an optimization approach. PCI Journal, v.44, n.2, p.40-53, mar/abr.

HEHL, M.E. (1986). Linguagem de programação estruturada: Fortran 77. São Paulo: McGraw-Hill.

HOROWITZ, B. (1993). Strength of slender concrete columns subjected to biaxial bending. In the World Congress on Optimal Design of Structural Systems, Rio de Janeiro, 1993. Anais. Rio de Janeiro COPPE/Federal University of Rio de Janeiro, v.1, p.395-402.

HUANCHUN, S.; ZHENG, C. (1985). Two-level optimum design of reinforced concrete frames with integer variables. Engineering Optimization, v.9, n.3, p.219-232, mar.

IMAM, M.H. (1998). Shape optimization of umbrella-shaped concrete shells subjected to self-weight as the dominant load. Computers and Structures, v.69, p.513-524.

JAMES, B.R. (1996). Probabilidade, um curso em nível intermediário. 2 ed. Rio de Janeiro: IMPA. 
JENKINS, W.M. (1992). Plane frame optimum design environment based on genetic algorithm. Journal of Structural Engineering, ASCE, v.118, n.11, p.3103-3112, nov.

JOHNSON, R.A.; WICHERN, D.W. Applied Multivariate Statistical Analysis. Englewood: Prentice Hall.

KANAGASUNDARAM, S.; KARIHALOO, B.L. (1991). Minimum-cost reinforced concrete beams and columns. Computers and Structures, v.41, n.3, p.509-518, mar.

KANDA, J.; ADACHI, T. (2001). Influence of probability distribution of loads on optimum reliability. Reliability Engineering and System Safety, v.73, p.197-204.

KOCER, F.Y.; ARORA, J.S. (1996). Design of prestressed concrete transmission poles: optimization approach. Journal of Structural Engineering, ASCE, v.122, n.7, p.804-814, jul.

KRAKOVSKI, M.B. (1997). Optimization of RC structures using design of experiments. Computers and structures, v.63, n.1, p.119-132.

KUHN, H.W.; TUCKER, A.W. (1951). Nonlinear programming. Proceedings of $2^{\text {nd }}$ Berkeley Symp. On Mathematics, Statistics and Probability, University of California Press, Berkeley, Calif. p.481-492.

LEITHOLD, L. (1990). O cálculo com geometria analítica. 3.ed. São Paulo: Harbra.

LI, C.Q.; MELCHERS, R.E. (1995). Failure probability of reinforced concrete columns under stochastic loads. Engineering Structures, v.17, n.6, p.419-424.

LIANG, Q.Q.; XIE, Y.M.; STEVEN, G.P. (2000). Optimal topology selection of continuum structures with displacement constraints. Computers and Structures, v.77, p.635-644. 
LIN, K.Y.; FRANGOPOL, D.M. (1996). Reliability based optimum design of reinforced concrete girders. Structural Safety, v.18, n.2, p.239-258.

LUENBERGER, D. (1984). Linear and nonlinear programming. 2.ed. Reading: Addison-Wesley.

LUNDBERG, J.E.; GALAMBOS, T.V. (1996). Load and resistence factor design of composite columns. Structural Safety, v.18, n.2, p.169-177.

LUO, X.; GRANDHI, R.V. (1996). Astros for reliability based multidisciplinary structural analysis and optimization. Computers e Structures, v.62, n.4, p. 737745.

MARDIA, K.V.; KENT, J.T.; BIBBY, J.M. Multivariate Analysis. New York: Academic Press.

MATOUS, K. et al. (2000). Applying genetic algorithms to selected topics commonly encountered in engineering practice. Compute. Methods Appl. Mech. Engineering, v.190, p.1629-1650.

MEYER, P.L. (1972). Probabilidade, Aplicações à Estatística. Rio de Janeiro: LTC.

MOOD, A.M.; GRAYBILL, F.; BOES, D.C.( 1986). Introduction to the Theory of Statistics. Nova York: McGraw-Hill.

MOHAMED, A.; SOARES, R.; VENTURINI, W.S. (2001). Partial safety factors for homogeneous reliability of non-linear reinforced concrete columns. Structural Safety, v.23, p.137-156.

MOHARRAMI, H.; GRIERSON, D.E. (1993). Computer-automated design of reinforced concrete frameworks. Journal of Structural Engineering, ASCE, v.119, n.7, p.2036-2058, jul. 
MONTGOMERY, D.C.; RUNGER, G.C. (2003). Estatística Aplicada e Probabilidade para Engenheiros. 2.ed. Rio de Janeiro: LTC.

MORRIS, A.J. (1982). Foundations of structural optimization: a unified approach. New York: John Wiley.

MORRISON, D.F. (1986). Multivariate Statistical Methods. New York: McGraw Hill.

NAAMAN, A.E. (1976). Minimum Cost versus minimum weight of prestressed slabs. Journal of Structural Division, ASCE, v.102, n.7, p.1493-1505, jul.

NAKANISHI, S.; NAKAYASU, H. (2002). Reliability design of structural system with cost effectiveness during life cycle. Computers and Industrial Engineering.

NIELSEN, T.; GUDMESTAD, O.T.; DALANE, J.I.; RETTEDAL, W.K.; AVENT, T. (1998). Utilization of principles from structural reliability in quantitative risk analysis: example from an offshore transport problem. Reliability Engineering and System Safety, v.61, n.1-2, p.127-137.

NOCEDAL, J.; WRIGHT, S.J. (1999). Numerical optimization. New York: Springer.

PANTELIDE, C.P.; BOOTH, B.C. (2000). Computer-aided design of optimal structures with uncertainty. Computers and structures, v.74, p.293-307.

POULSEN, N.P.; DAMKILD, L. (2000). Limit state analyses of reinforced concrete plates subjected to in-plane forces. International journal of solids and structures, v.37, p.6011-6029.

PRAGER, W. (1968). Optimality in structural design. Proceedings of the National Academy of Sciences of the USA, University of California (San Diego), v.61, n.3, p.794-796. 
PU, Y.; DAS, P.K.; FAULKNER, D. (1996). A strategy for reliability based optimization. Engineering Structures, v.19, n.3, p.276-282.

RAMAN, A. (1990). A non-iterative method of structural optimization for static, dynamic and response problems. Computers and Structures, v.34, n.2, p.185190.

RATH, D.P.; AHLAWAT, A.S.; RAMASWAMY, A. (1999). Shape optimization of RC flexural members. Journal of Structural Engineering, ASCE, v.125, n.12, p.1439-1446, dez.

RIZZI, P. (1976). Optimization of multi-constrained structures based on optimality criteria. Proceedings of AIAA/ASME/SAE $17^{\text {th }}$ Struct. Dyn. and Mat. Conference, King of Prussia, Pa.

ROYSET, J.O.; KIUREGHIAN, A.D.; POLAK, E. (2001). Reliability based optimal structural design by the decoupling approach. Reliability Engineering and System Safety, v.73, p.213-221.

SCHMIDT, L.A. (1960). Structural design by systematic synthesis. Proceedings, ASCE, $2^{\text {nd }}$ Conference on Electronic Computation, Pittsburgh, Pa, p.105-132.

SHIH, C.J. (1997). Fuzzy and improved penalty approaches for multiobjective mixed-discrete optimization in structural systems. Computers and structures, v.63, n.3, p.559-565.

SINGH, R.; KOENKE, C. (2000). Simulation framework for risk assessment of damage tolerant structures. Computers and Structures, v.77, p.101-115.

SOARES, R.C. (1997). Otimização de seções transversais de concreto armado sujeitas à flexão: aplicação a pavimentos. Dissertação (Mestrado) - Escola de Engenharia de São Carlos, Universidade de São Paulo, São Carlos. 1997. 
SOARES, R.C. (2001). Um estudo sobre modelos mecânico-probabílisticos para pórticos de concreto armado. Tese (Doutorado) - Escola de Engenharia de São Carlos, Universidade de São Paulo, São Carlos. 2001.

SOARES, R.C.; MOHAMED, A.; VENTURINI, W.S.; LEMAIRE, M. (2002). Reliability analysis of non-linear reinforced concrete frames using the response surface method. Reliability Engineering and System Safety, v.75, p.1-16.

SOUZA, J.B.M.; VAZ, L.E. (1993). Otimização do dimensionamento de pilar padrão de concreto armado com seção retangular. III Simpósio EPUSP sobre Estruturas de Concreto. São Paulo. p.393-405.

SPIEGEL, M.L. (1978). Probabilidade e Estatística. Coleção Schaum. New York: McGraw-Hill.

SPIRES, D.; ARORA, J.S. (1990). Optimal design of tall RC-framed tube buildings. Journal of Structural Engineering, ASCE, v.116, n.4, p.877-897, abr.

UNIVERSIDADE DE SÃO PAULO. Escola de Engenharia de São Carlos. Serviço de Biblioteca (2002). Diretrizes para elaboração de dissertações e teses na EESC-USP. 3.ed. São Carlos.

VATN, J. (1998). A discussion of the acceptable risk problem. Reliability Engineering and System Safety, v.61, n.1, p.11-19.

VENKAYYA, V.B; KHOT, N.S.; REDDY, V.S. (1968). Energy distribution in an optimum design. AFFDL-TR-68-156, Flight Dynamics Laboratory, Wright Patterson AFB, Ohio.

VIANNA, L.C.C. (2003). Otimização de seções transversais de concreto armado: aplicação a pórticos. Dissertação (Mestrado) - Escola de Engenharia de São Carlos, Universidade de São Paulo, São Carlos. 2003. 
WEN, Y.K. (2001). Minimum lifecycle cost design under multiple hazards. Reliability Engineering and System Safety, v.73, p.223-231.

ZIELINSKI, Z.A.; LONG, W.; TROITSKY, M.S. (1995). Designing reinforced concrete short-tied columns using the optimization technique. ACI Structural Journal, v.92, n.5, p.619-626, set/out. 